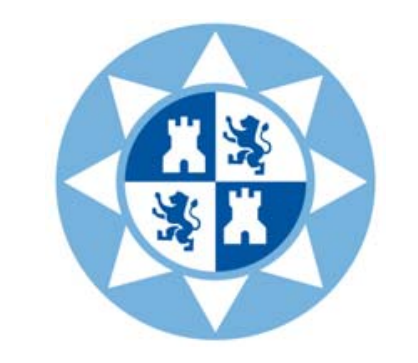

UNIVERSIDAD POLITÉCNICA DE CARTAGENA

\title{
CONTRIBUCIÓN AL DESARROLLO DE SISTEMAS DE TELELECTURA INTELIGENTE CON IOT
}

\author{
TESIS DOCTORAL
}

\author{
Autor \\ D. Jesús Rubio Aparicio \\ Directores \\ Dr. D. Juan Suardíaz Muro \\ Dr. D. José Fernando Cerdán Cartagena
}





\section{CONFORMIDAD DE DEPÓSITO DE TESIS DOCTORAL POR LA COMISIÓN ACADÉMICA DEL PROGRAMA}

$D^{a}$. Sonia Busquier Sáez, Secretaría de la Comisión Académica del Programa Tecnologías Industriales.

\section{INFORMA:}

Que la Tesis Doctoral titulada, "CONTRIBUCIÓN AL DESARROLLO DE SISTEMAS DE TELELECTURA INTELIGENTE CON IOT", ha sido realizada, dentro del mencionado Programa de Doctorado, por D. Jesús Rubio Aparicio, bajo la dirección y supervisión del Dr. Juan Suardíaz Muro y del Dr. José Fernando Cerdán Cartagena.

En reunión de la Comisión Académica, visto que en la misma se acreditan los indicios de calidad correspondientes y la autorización del Director de la misma, se acordó dar la conformidad, con la finalidad de que sea autorizado su depósito por el Comité de Dirección de la Escuela Internacional de Doctorado.

La Rama de conocimiento por la que esta tesis ha sido desarrollada es:

\section{Ciencias}

Ciencias Sociales y Jurídicas

$\searrow$ Ingeniería y Arquitectura

En Cartagena, a 12 de junio de 2019

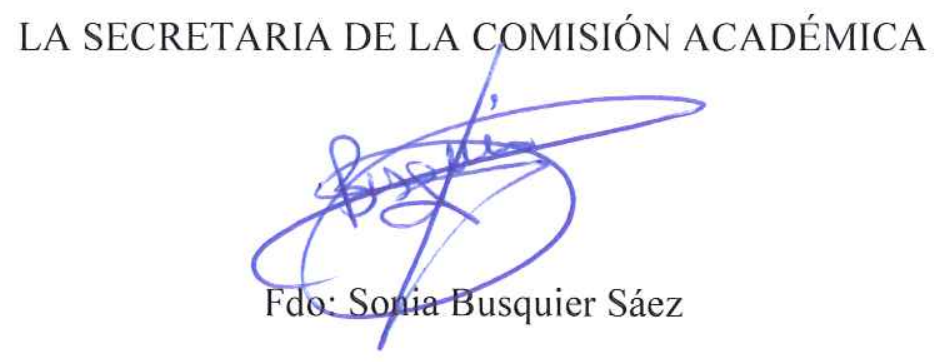





\section{Universidad}

Politécnica

de Cartagena

DT-16

\section{CONFORMIDAD DE SOLICITUD DE AUTORIZACIÓN DE DEPÓSITO DE}

TESIS DOCTORAL POR EL DIRECTOR DE LA TESIS

D. Juan Suardíaz Muro Director de la Tesis doctoral CONTRIBUCIÓN AL DESARROLLO DE SISTEMAS DE TELELECTURA INTELIGENTE CON IOT.

\section{INFORMA:}

Que la referida Tesis Doctoral, ha sido realizada por D. Jesús Rubio Aparicio, dentro del Programa de Doctorado Tecnologías Industriales, dando mi conformidad para que sea presentada ante el Comité de Dirección de la Escuela Internacional de Doctorado para ser autorizado su depósito.

La rama de conocimiento en la que esta tesis ha sido desarrollada es:

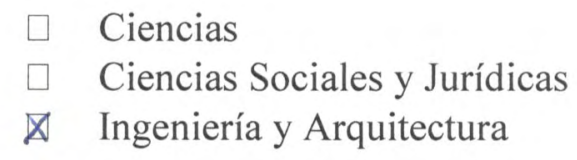

En Cartagena, a 12 de junio de 2019

EL DIRECTOR DE LA TESIS

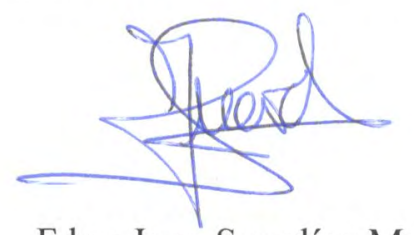

Fdo.: Juan Suardíaz Muro 



\section{CONFORMIDAD DE SOLICITUD DE AUTORIZACIÓN DE DEPÓSITO DE} TESIS DOCTORAL POR EL DIRECTOR DE LA TESIS

D. José Fernando Cerdán Cartagena Director de la Tesis doctoral CONTRIBUCIÓN AL DESARROLLO DE SISTEMAS DE TELELECTURA INTELIGENTE CON IOT.

\section{INFORMA:}

Que la referida Tesis Doctoral, ha sido realizada por D. Jesús Rubio Aparicio, dentro del Programa de Doctorado Tecnologías Industriales, dando mi conformidad para que sea presentada ante el Comité de Dirección de la Escuela Internacional de Doctorado para ser autorizado su depósito.

La rama de conocimiento en la que esta tesis ha sido desarrollada es:

$\square$ Ciencias

$\square$ Ciencias Sociales y Jurídicas

\ Ingeniería y Arquitectura

En Cartagena, a 12 de junio de 2019

EL DIRECTOR DE LA TESIS

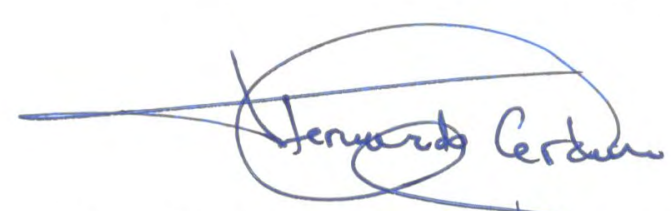

Fdo.: José Fernando Cerdán Cartagena 



\section{Agradecimientos}

A todas aquellas personas que a lo largo de mi vida han alimentado de una

forma u otra mi interés por descubrir cómo funcionan las cosas y por materializar mis propias ideas. En especial, a mis padres, mi abuelita, mi padrino y mi tato. 

Internet of Things (IOT) o Internet de las Cosas está cobrando importancia y adquiriendo una participación cada vez mayor en todo ámbito a nivel mundial. Esta tendencia no aplica solo a personas en su vida cotidiana, sino que se convierte también en un factor determinante para las empresas. Lo que hace unos años era una tecnología potencial para habilitar nuevas iniciativas de negocio y mejoras operacionales, hoy en día ha pasado a la fase de implementación donde es posible interconectar un elevado número de dispositivos. La conexión de tal ingente cantidad de objetos prevista, supone nuevos retos para las redes que les den soporte, debiendo tener una alta escalabilidad para absorber el crecimiento exponencial previsto y simplicidad en el registro de nuevos dispositivos. Además, son factores críticos la autonomía, el alcance de la señal transmitida y el ancho de banda. Bajo esta premisa, la tecnología de red que mejor se adapta a los requisitos descritos es LPWAN (Low Power Wide Area Network), que describe redes de área amplia y baja potencia específicas para IoT.

En este trabajo nos apoyamos en dos de los sistemas más populares de comunicaciones LPWAN para redes IoT: Sigfox y LoRa. Para ello, se ha realizado un estudio de las capacidades y prestaciones de cada uno de ellos, así como un análisis de cobertura en casos reales. Basándose en estas tecnologías, se ha diseñado una arquitectura propia de red mixta y flexible, capaz de combinar diferentes soluciones de comunicación IOT, que permite la conexión de objetos loT sin la restricción de la tecnología empleada y capaz de adaptarse durante el despliegue a las necesidades de cada aplicación. Con esta solución se habilita la coexistencia tanto de dispositivos Sigfox como LoRaWAN y queda abierta la posibilidad de otras tecnologías. Además se facilita la interoperabilidad tanto de los dispositivos desarrollados en este trabajo, como los comercialmente disponibles aunque provengan de diferentes fabricantes.

La arquitectura propuesta se ha validado sobre un caso real aplicado a la telelectura y telecontrol en contadores de consumo de agua residencial. Se ha llevado a cabo el diseño, implementación y evaluación de una solución especializada en el manejo de agua residencial mediante el desarrollo y conexión de dispositivos inteligentes de medición de consumo de agua integrados en una arquitectura IoT mixta LoRaWAN-Sigfox. Esta solución permite hacer una gestión eficiente de la red de distribución. A diferencia de la mayoría de los sistemas actuales, y haciendo uso de comunicación bidireccional, permite, además de la telelectura, actuar a distancia sobre el equipo del cliente interrumpiendo el suministro en caso de incidencias. La telelectura inteligente proporciona un mayor volumen de datos sobre el agua consumida, lo que posibilita un ahorro en el consumo de agua contribuyendo al desarrollo de la directiva para el agua de la Unión Europea y a un mejor desarrollo de las Smart Cities o ciudades inteligentes.

Los resultados obtenidos durante el despliegue y con respecto a un sistema de telelectura en servicio en banda ISM de $169 \mathrm{MHz}$, demuestran que se ha mejorado la tasa de recepción de datos aplicando tecnologías LPWAN para IoT, lo que convierte a estos dispositivos en una alternativa viable frente a soluciones comerciales disponibles. Se ofrece una solución innovadora y de bajo coste para ampliar el área de cobertura de la red a zonas de sombra en ubicaciones adversas utilizando también tecnologías loT. 



\section{SUMMARY}

The "Internet of Things" (IOT) is gaining importance and increasing participation in every field worldwide. This trend does not only apply to people in their daily lives, but also becomes a determining factor for companies. What some years ago was a potential technology of enabling new digital business initiatives and operational improvements, today it has evolved to the implementation phase where is possible to interconnect a large number of devices. The planned connection of such an enormous amount of objects, envisages new challenges for the networks supporting them, and must have a high scalability to absorb the expected exponential growth as well as simplicity in the registration of new devices. Network coverage, energy consumption of devices and transmission bandwidth, are critical factors to be considered. Under this premise, the network technology that best adapts to the described requirements is LPWAN (Low Power Wide Area Network), which describes wide area and low power networks specific for loT applications.

In this work we rely on two of the most popular LPWAN communication systems for loT networks, such as Sigfox and LoRa. To this end, a study of the capacities and benefits of each of them has been carried out, as well as a coverage analysis in real cases. Based on these technologies, an own mixed and flexible network architecture has been designed. It is able to combine different loT communication solutions and allows the connection of IOT objects without the restriction of the used technology. In this way it can be adapted during the deployment to the needs of each application. With this solution the coexistence of both device types, Sigfox and LoRaWAN, is enabled and the possibility of other technologies is open. It also facilitates the interoperability of both, the devices developed in this work, and the commercially available from different manufacturers.

The proposed architecture has been validated in a real case study applied to remote metering and remote control over water supply service. The design, implementation and evaluation of a specialized solution in residential water management has been carried out through the development and connection of smart water meter devices integrated in a mixed SigfoxLoRaWAN IoT architecture. This solution allows efficient management of the distribution network. Unlike most of the current systems, and making use of bidirectional communication, it allows, in addition to remote reading, to act remotely on the client's equipment interrupting the supply in case of incidents. Smart remote metering provides a greater volume of data on the water consumed, which allows savings in water consumption, contributing to the development of the directive for water efficiency in the European Union and to a better development of Smart Cities.

The results obtained during the deployment regarding to a system of remote metering in service in the ISM band of $169 \mathrm{MHz}$, show that the reception data rate has been improved by applying LPWAN technologies for IOT, which makes these devices a viable alternative to other available commercial solutions. An innovative and low-cost solution is offered to extend the coverage area of the network within shadow areas in adverse locations, also using loT technologies. 



\begin{tabular}{|c|c|}
\hline ABP & Activation By Personalization \\
\hline ABS & Acrylonitrile Butadiene Styrene \\
\hline ACK & Acknowledgement \\
\hline API & Application Programming Interface \\
\hline ASCll & American Standard Code for Information Interchange \\
\hline BPS & Bits Per Second \\
\hline BPSK & Binary Phase Shift Keying \\
\hline CRC & Cyclic Redundancy Check \\
\hline CSS & Chirp Spread Spectrum \\
\hline DBPSK & Diferential Binary Phase Shift Keying \\
\hline ECC & Error Ccorrecting Code \\
\hline EDI & Edificio de Laboratorios de Investigación \\
\hline ERP & Effective Radiated Power \\
\hline FSK & Frequency Shift Keying \\
\hline GFSK & Gaussian Frequency Shift Keying \\
\hline GHZ & Giga Hertzio \\
\hline HITP & HyperText Transfer Protocol \\
\hline HTIPS & HyperText Transfer Protocol Secure \\
\hline ID & Identifier \\
\hline IDE & Integrated Development Environment \\
\hline IOT & Internet Of Things \\
\hline IP & Ingress Protection \\
\hline
\end{tabular}




\begin{tabular}{|c|c|}
\hline IP & Internet Protocol \\
\hline ISM & Industrial Scientific Medical \\
\hline $\mathbf{K H Z}$ & Kilo Hertz \\
\hline LNA & Low Noise Amplifier \\
\hline LORA & Long Range \\
\hline LORAWAN & Long Range Wide Area Network \\
\hline LPWAN & Low Power Wide Area Network \\
\hline MAC & Medium Access Control \\
\hline MHZ & Mega Hertz \\
\hline OOB & Out Of Band \\
\hline OSI & Open System Interconnection \\
\hline OTAA & Over The Air Activation \\
\hline PAC & Porting Authorisation Code \\
\hline REST & Representational State Transfer \\
\hline RF & Radio Frequency \\
\hline RSMA & Reverse SubMiniature A-version \\
\hline RSSI & Received Signal Strength Indicator \\
\hline SAAS & Software As A Service \\
\hline SMA & SubMiniature A-version \\
\hline SNR & Signal Noise Ratio \\
\hline UNX & Uniplexed Information and Computing Service \\
\hline UPCT & Universidad Politécnica de Cartagena \\
\hline USB & Universal Serial Bus \\
\hline VPN & Virtual Private Network \\
\hline WF & Wireless Fidelity \\
\hline
\end{tabular}




\section{ÍNDICE DE CONIENIDO}

CAPÍTULO 1 INTRODUCCIÓN.

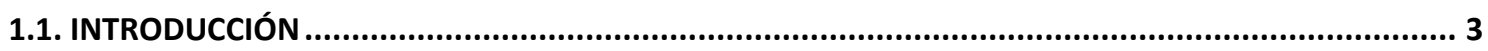

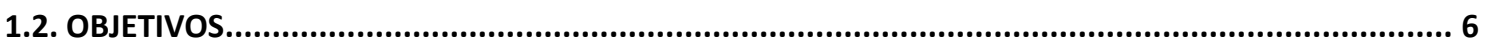

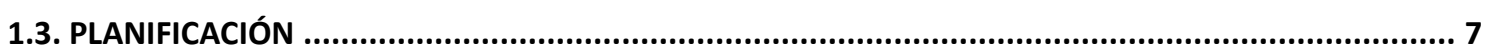

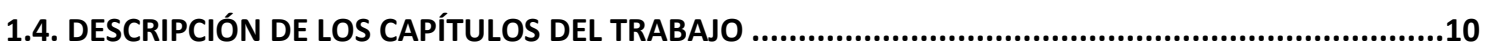

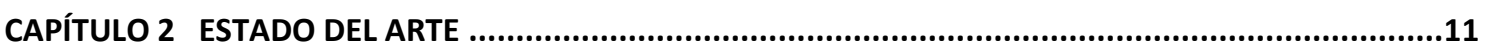

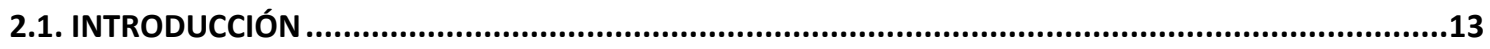

2.2. SOLUCIONES PARA LECTURA AUTOMÁTICA DE CONSUMO RESIDENCIAL DE AGUA......................13

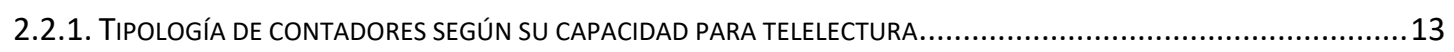

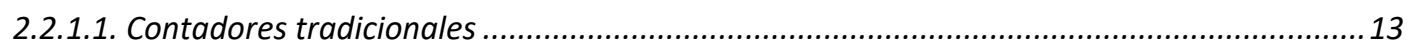

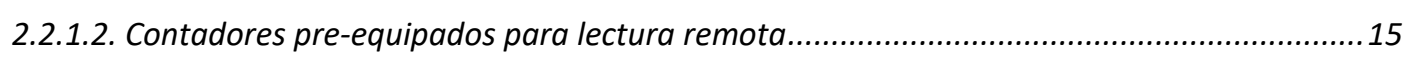

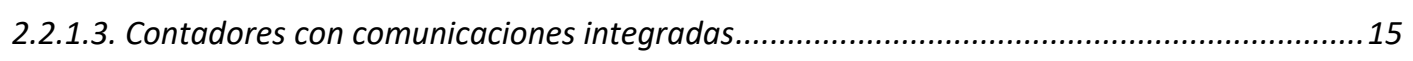

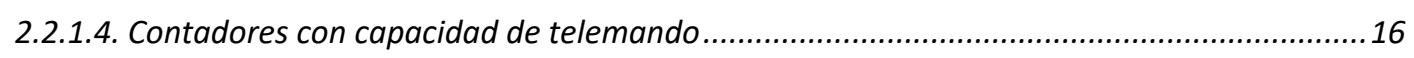

2.2.2. DISPOSITIVOS ACOPLABLES A CONTADOR TRADICIONAL PARA REALIZAR TELELECTURA.....................................17

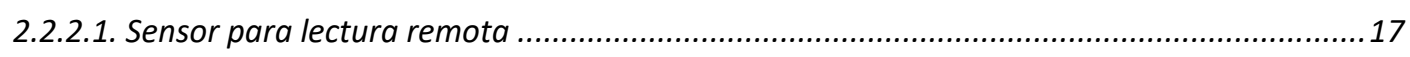

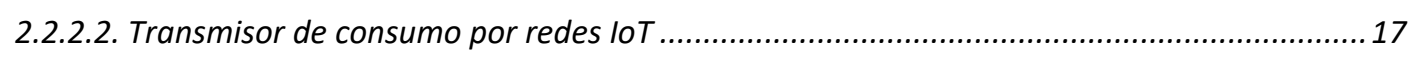

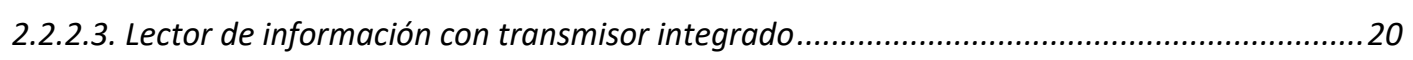

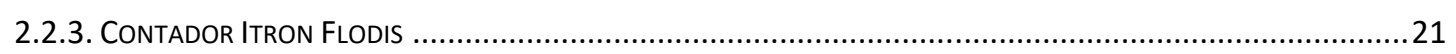

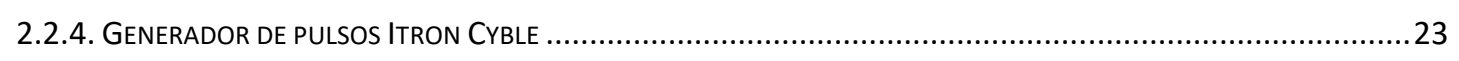

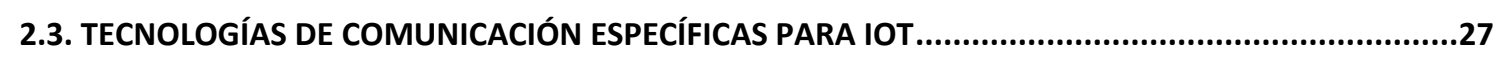

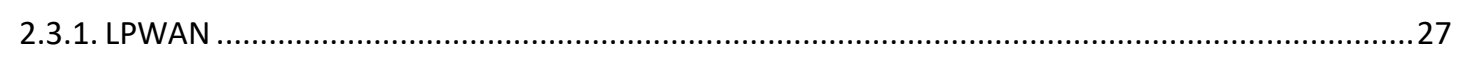

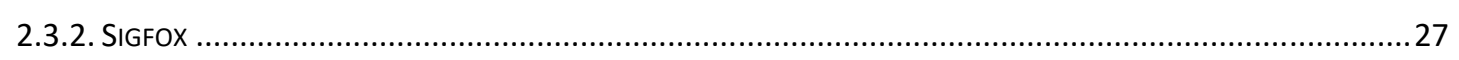

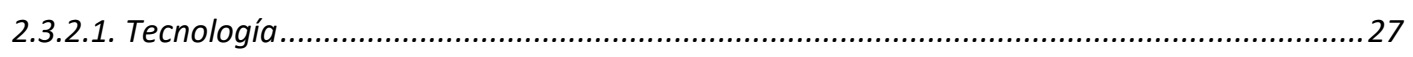

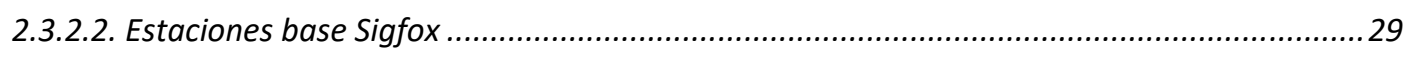

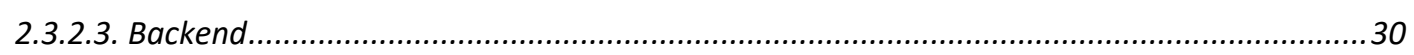

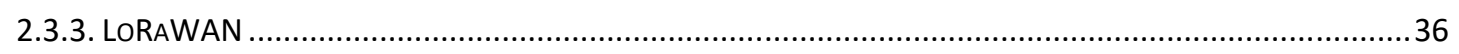

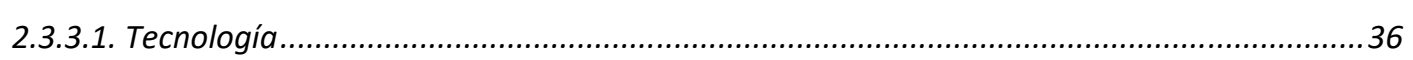

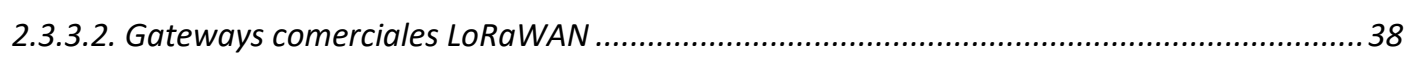

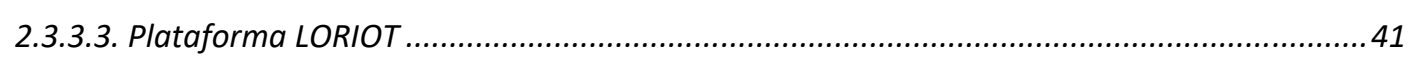

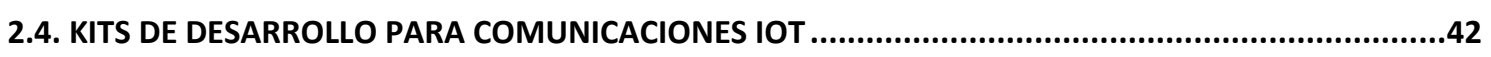

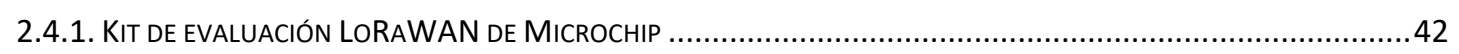

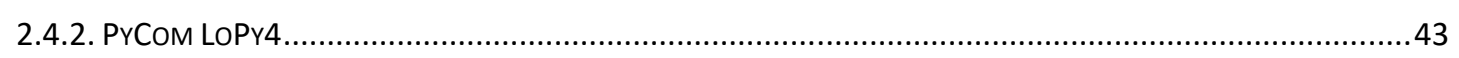

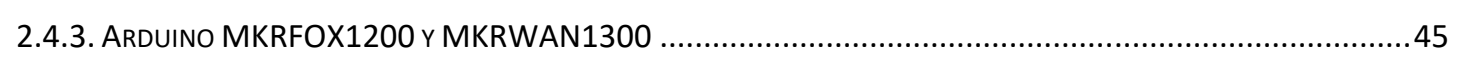

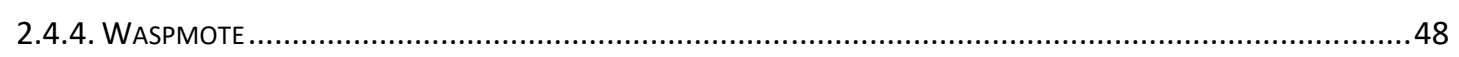

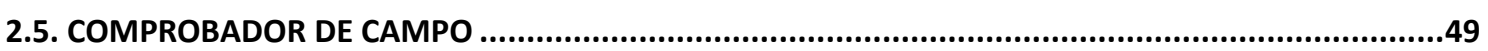

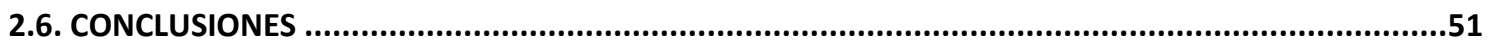




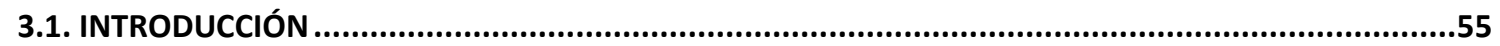

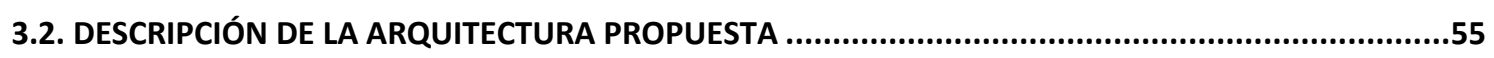

3.3. IMPLEMENTACIÓN HARDWARE

3.3.1. DISPOSITIVO DE TELEMEDIDA Y TELECONTROL CON WASPMOTE ........................................................56

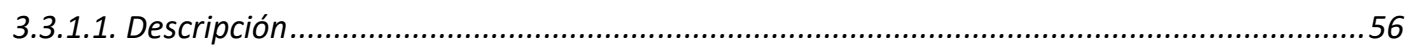

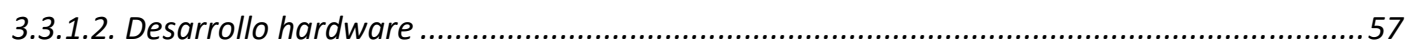

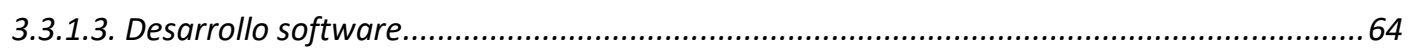

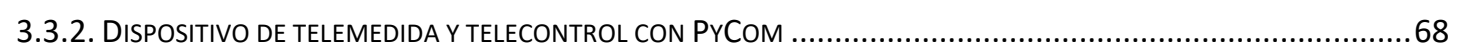

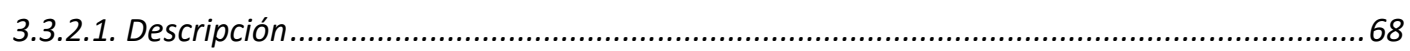

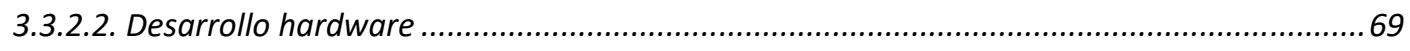

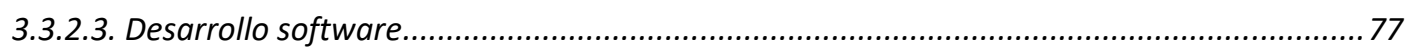

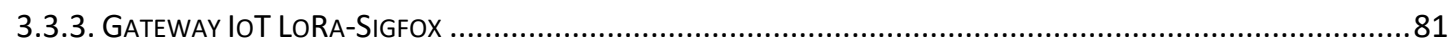

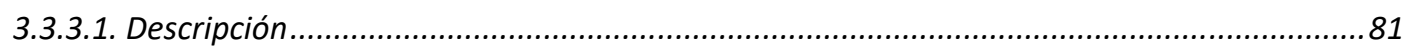

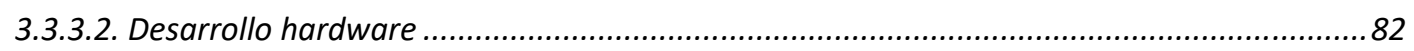

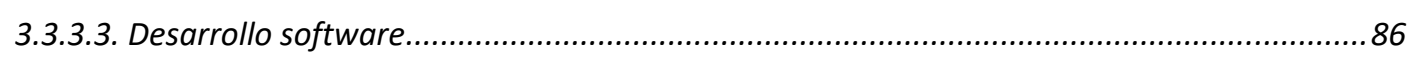

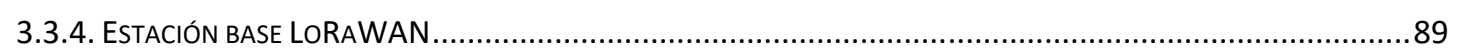

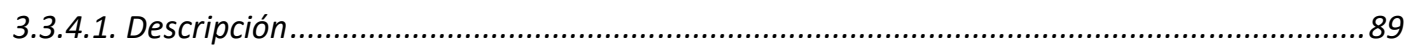

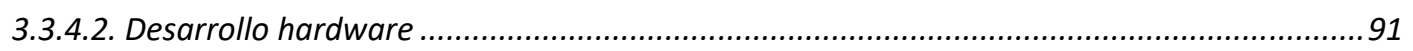

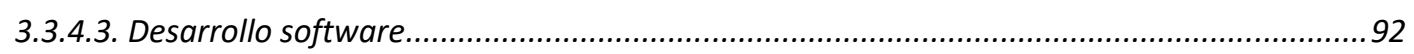

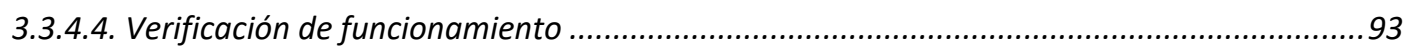

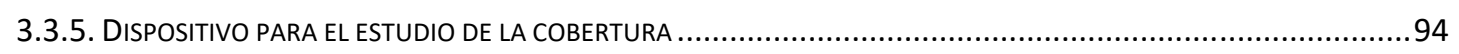

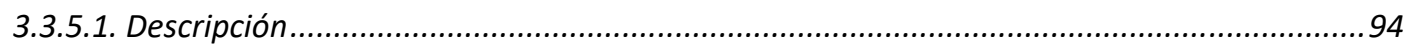

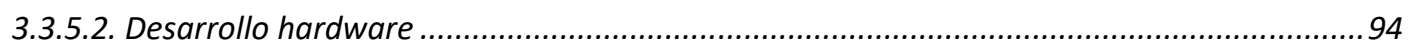

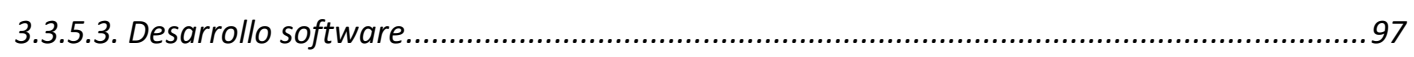

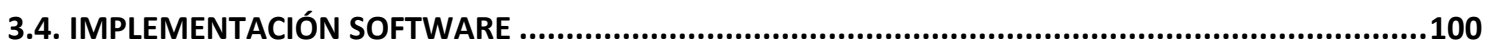

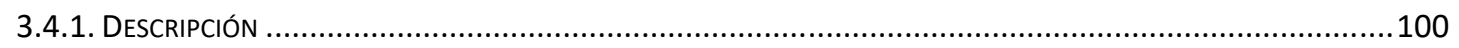

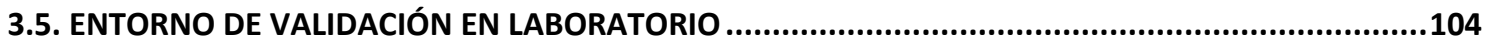

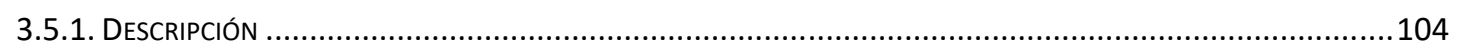

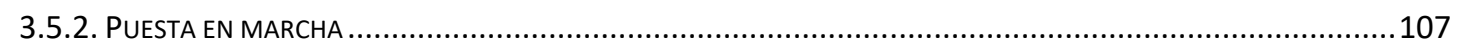

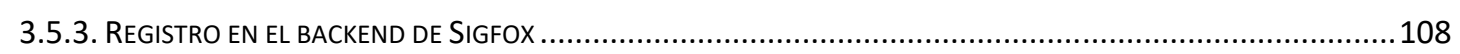

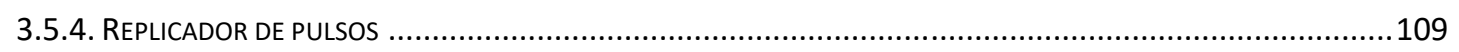

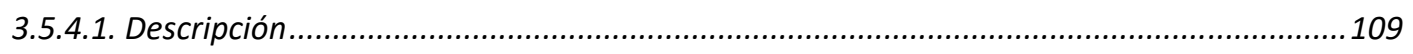

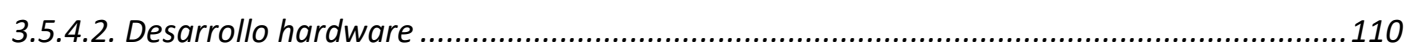

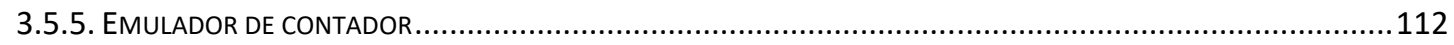

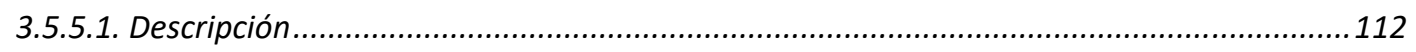

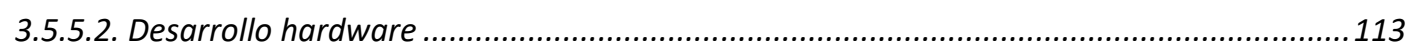

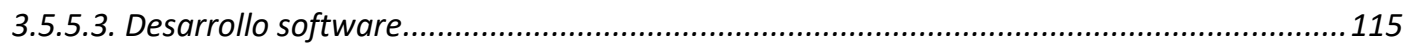

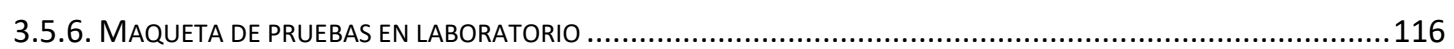

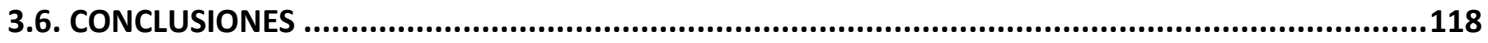




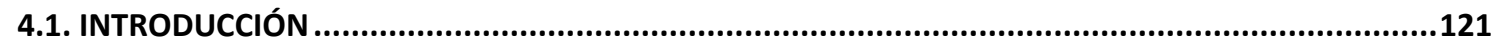

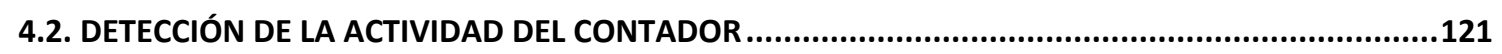

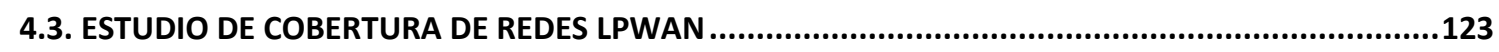

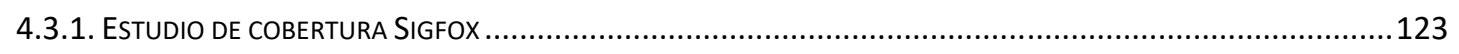

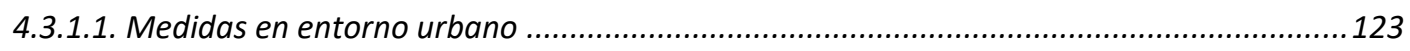

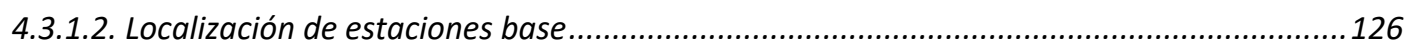

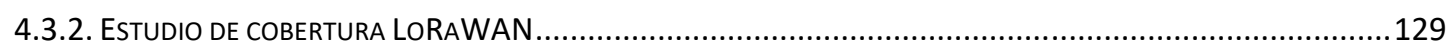

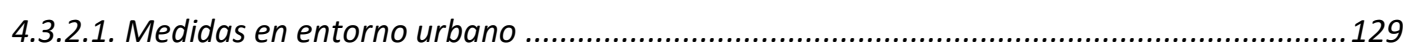

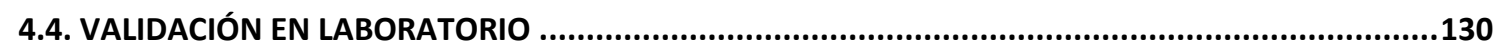

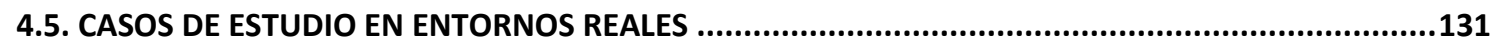

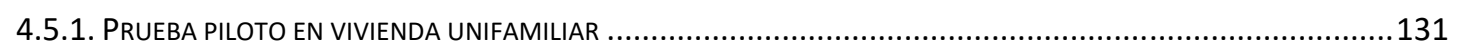

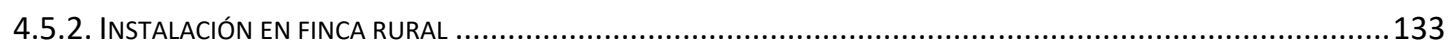

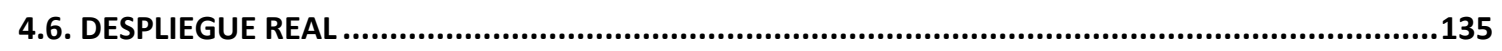

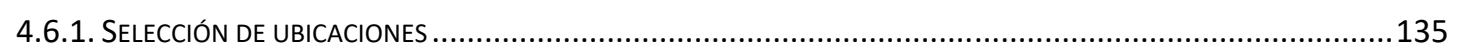

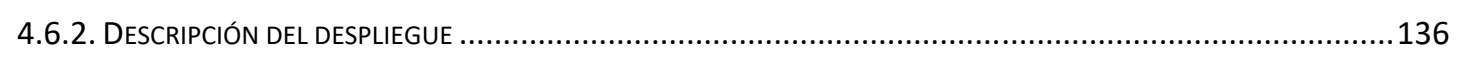

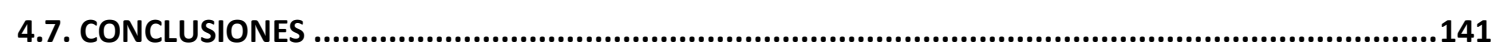

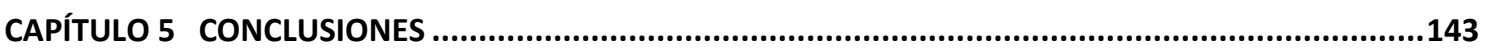

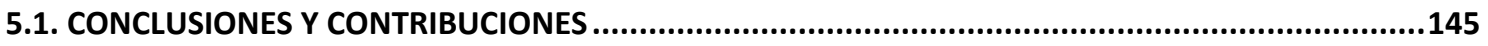

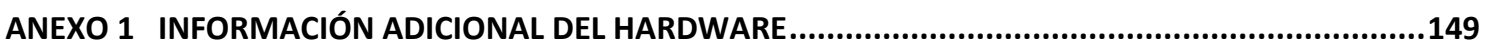

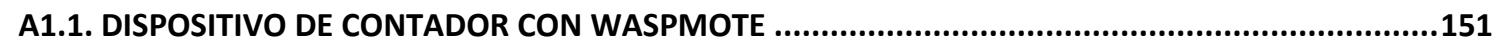

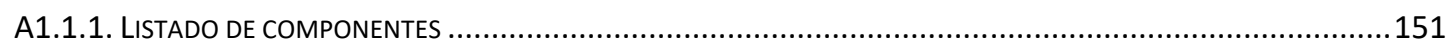

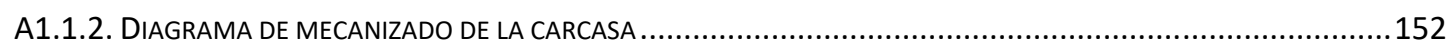

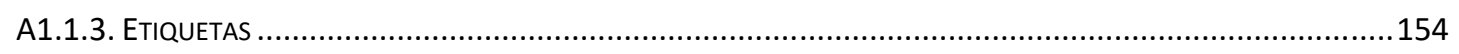

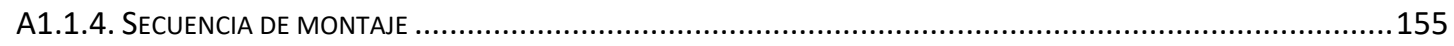

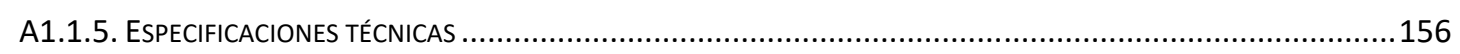

A1.2. DISPOSITIVO DE CONTADOR CON PYCOM ...................................................................157

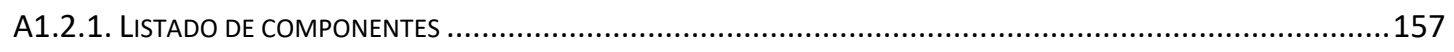

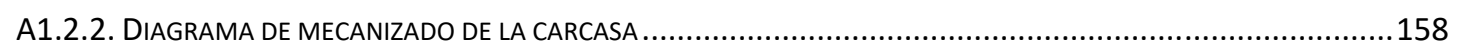

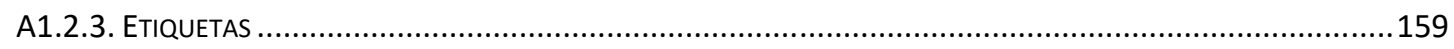

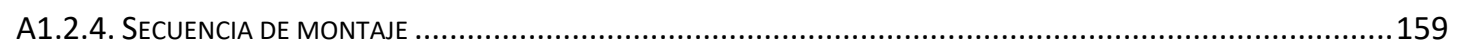

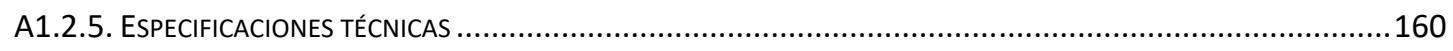

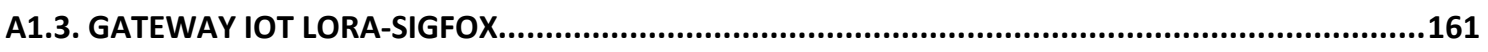

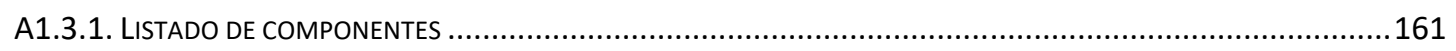

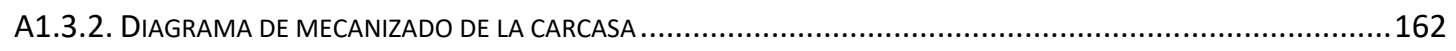

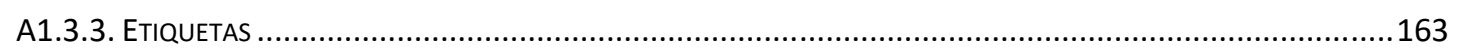

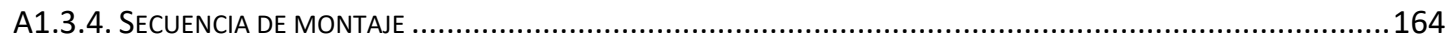

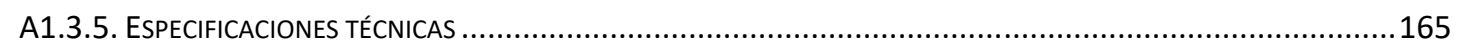

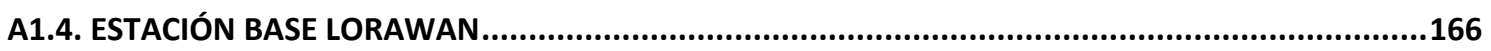

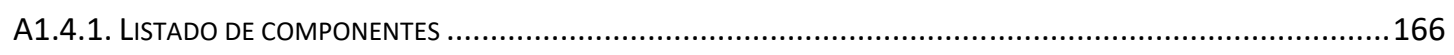

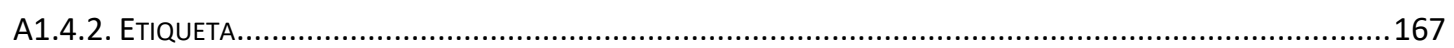




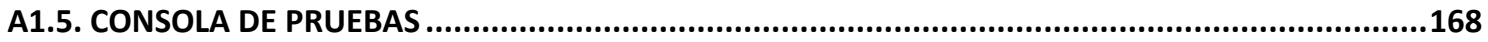

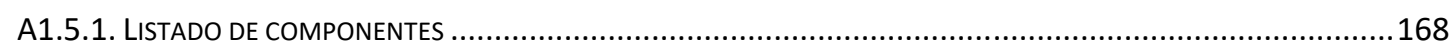

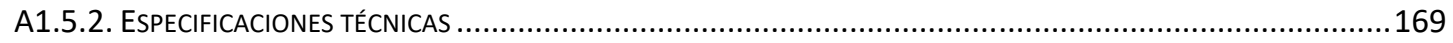

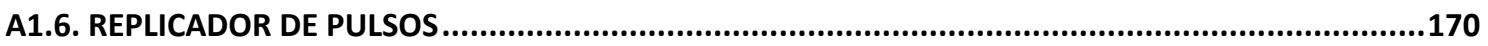

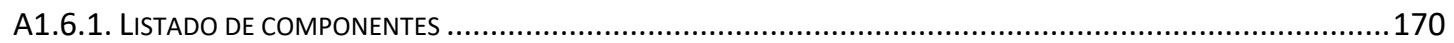

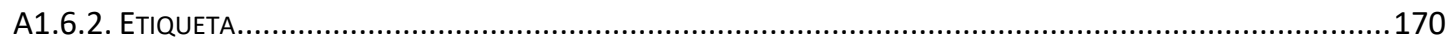

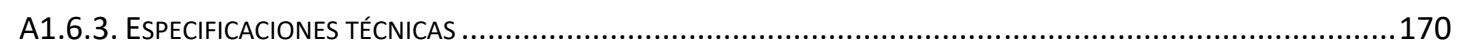

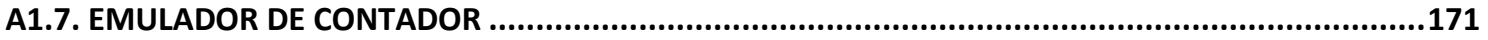

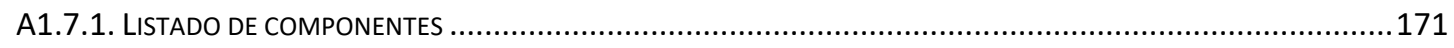

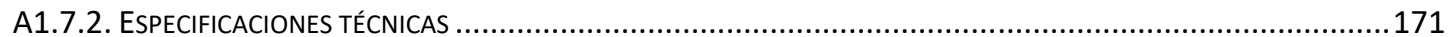

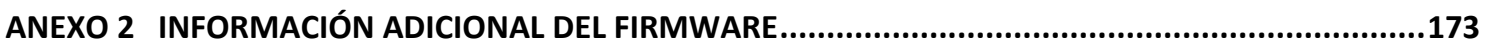

A2.1. DISPOSITIVO DE CONTADOR CON WASPMOTE ........................................................175

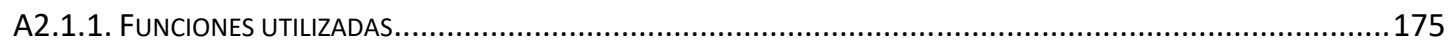

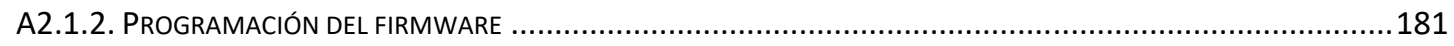

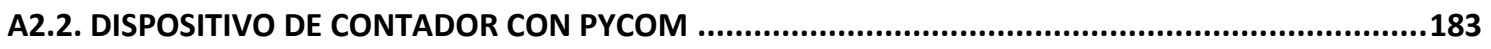

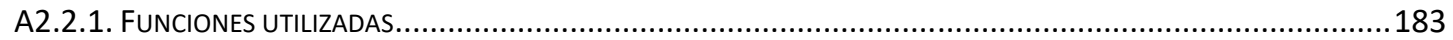

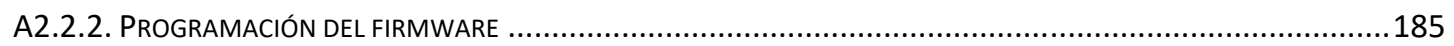

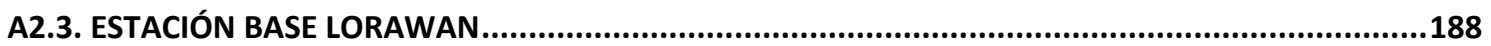

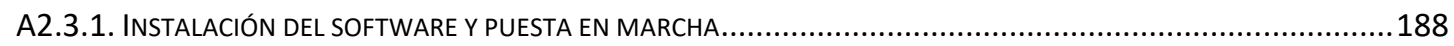

A2.4. ENTORNO DE VALIDACIÓN EN LABORATORIO ............................................................189

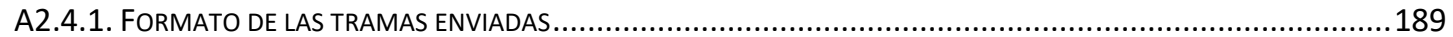

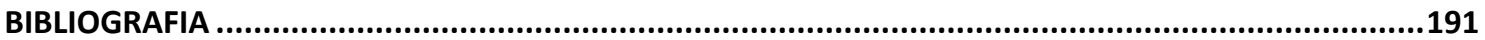




\section{ÍNDICEDERGURAS}

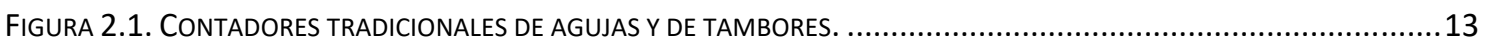

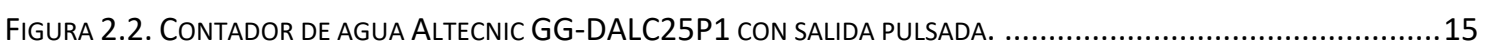

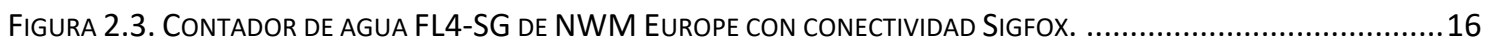

FIGURA 2.4. CONTADOR CON VÁLVULA HYDROKONEKT SMART DE HYDROKO. ................................................. 16

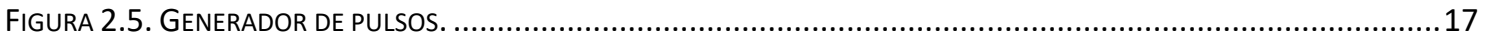

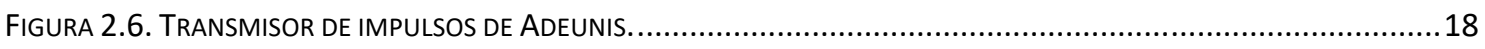

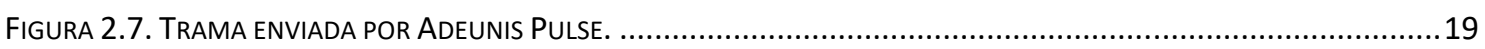

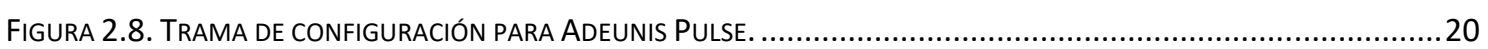

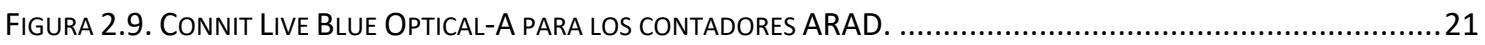

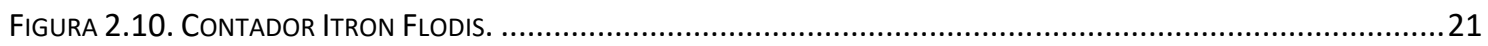

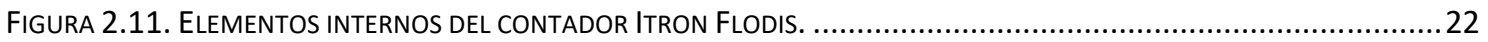

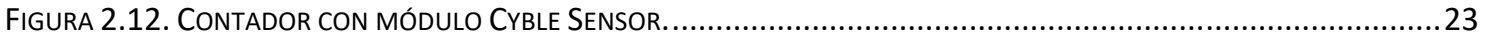

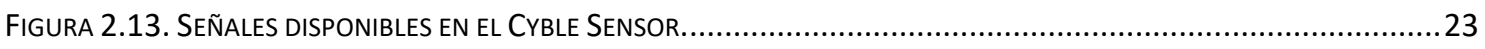

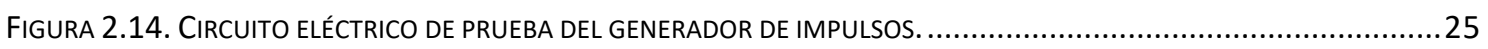

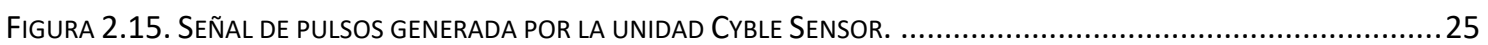

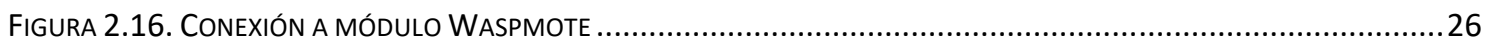

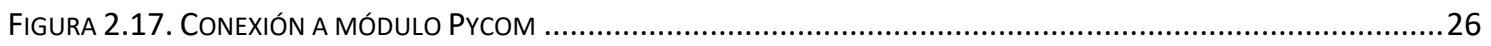

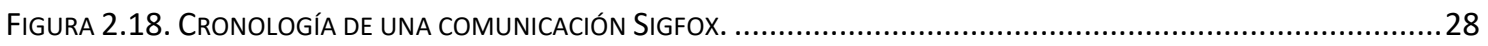

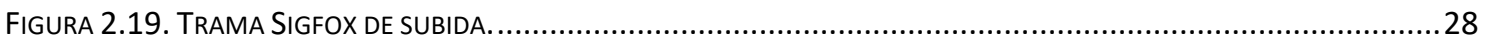

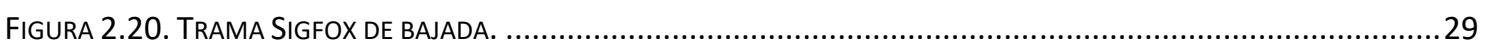

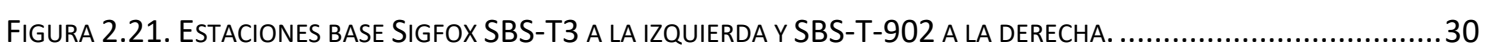

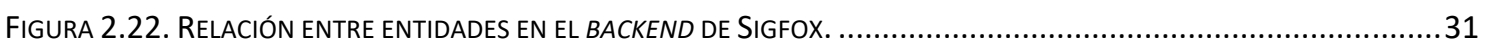

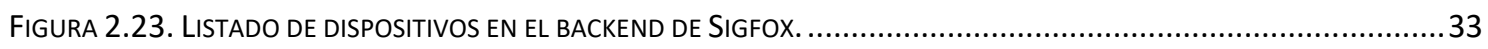

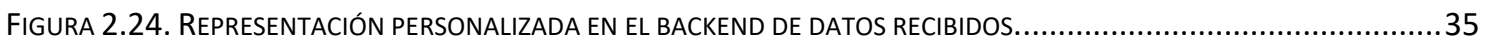

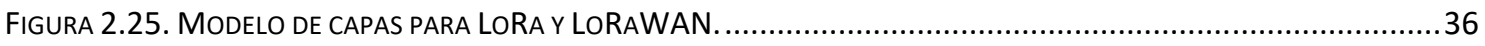

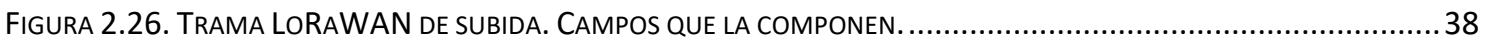

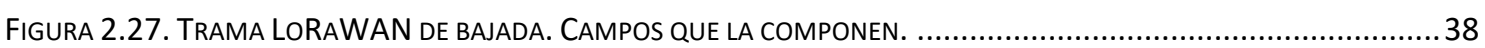

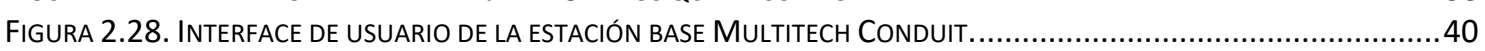

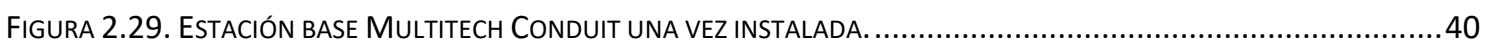

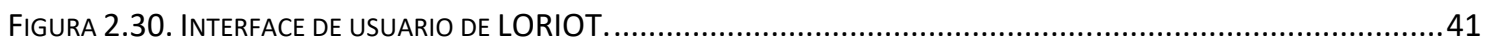

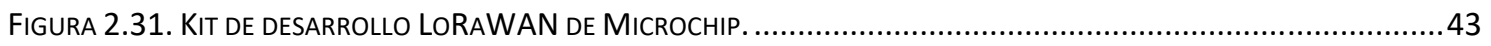

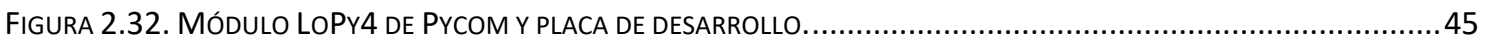

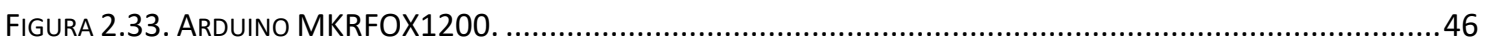

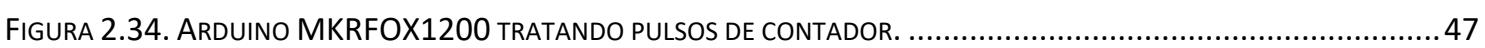

FIGURA 2.35. DATOS RECIBIDOS EN EL BACKEND DE SIGFOX DEL MKRFOX1200 ................................................4

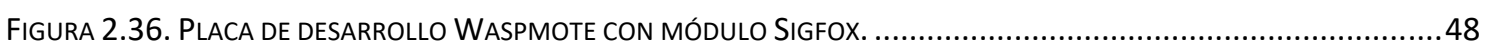

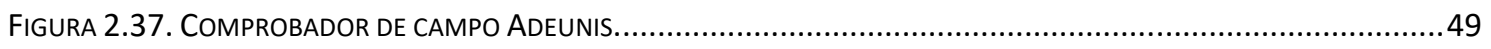

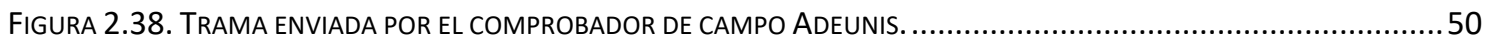

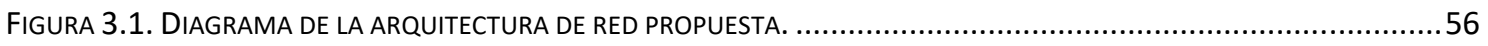

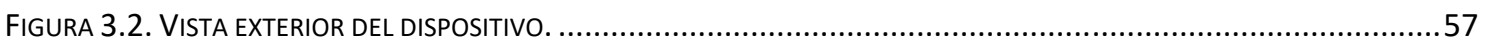

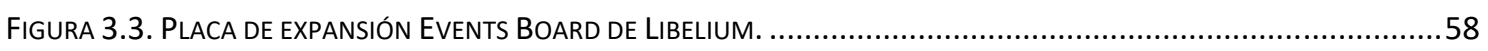

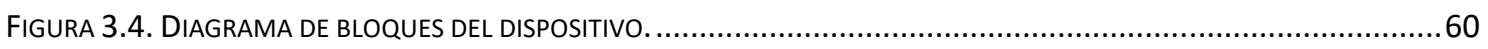

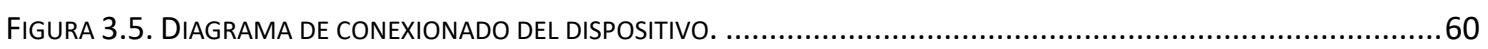

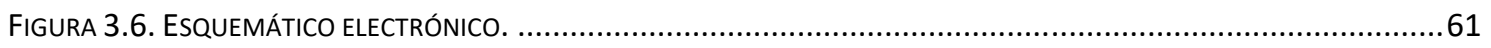

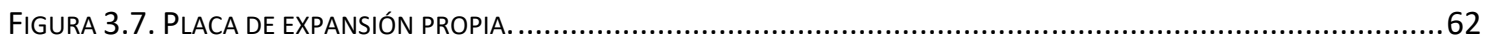

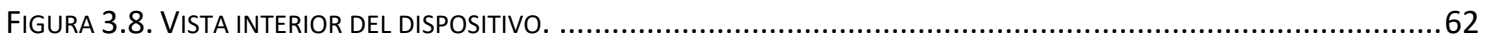

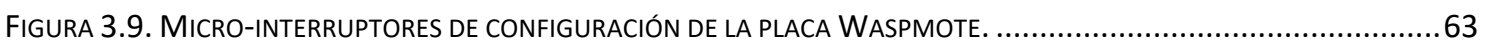

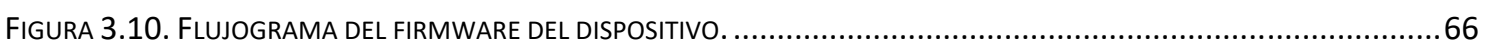

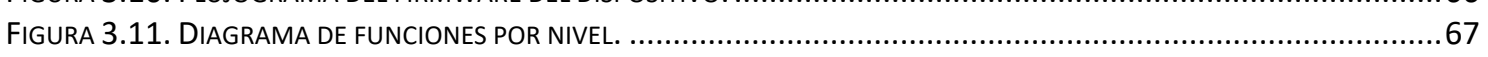

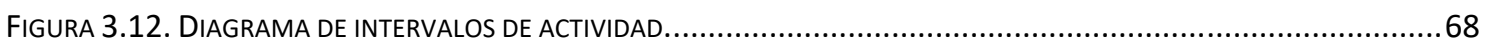

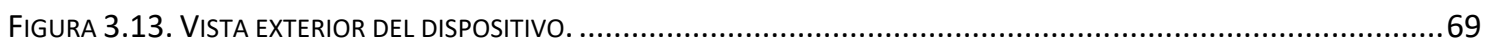

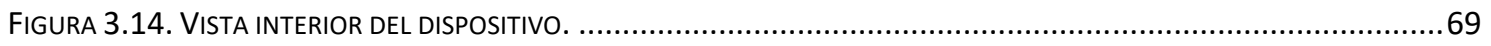




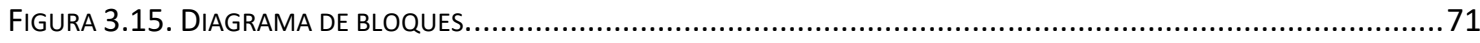

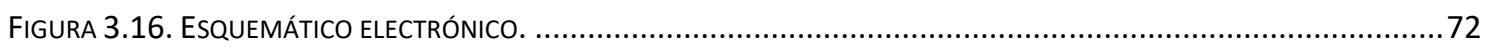

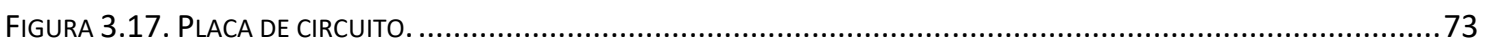

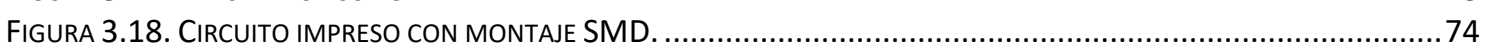

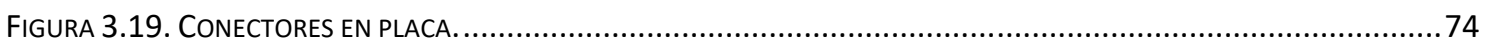

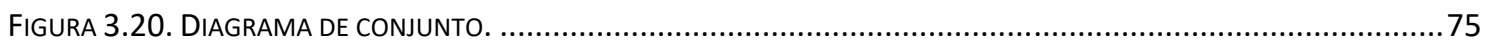

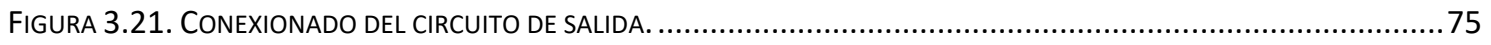

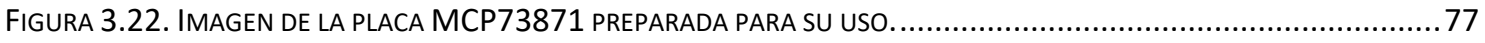

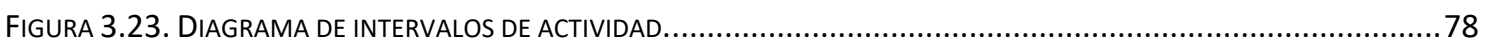

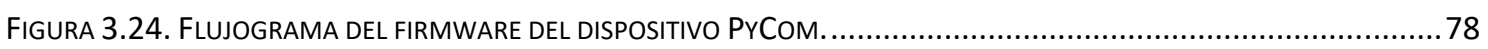

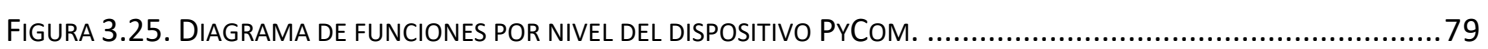

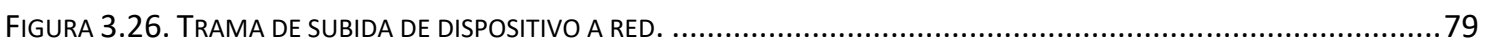

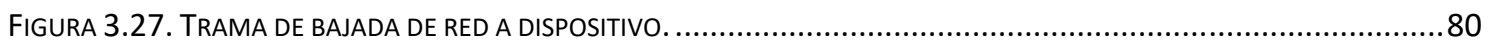

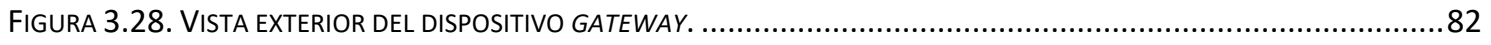

FIGURA 3.29. VISTA INTERIOR DEL DISPOSITIVO GATEWAY Y CONEXIÓN DE PANEL SOLAR. ..........................................8 83

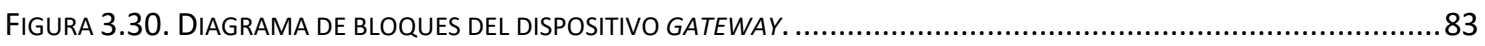

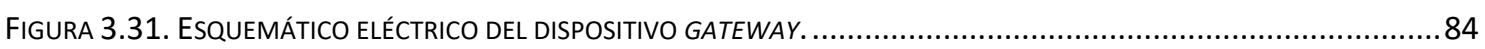

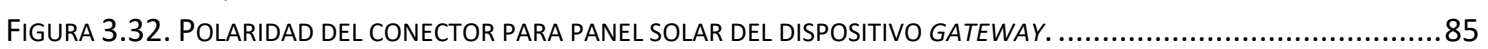

FIGURA 3.33. MICRO-INTERRUPTORES DE CONFIGURACIÓN DE PLACA WASPMOTE. ...................................................8

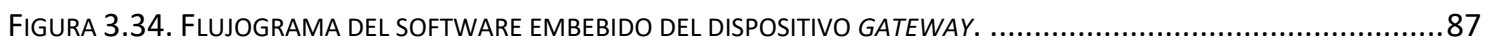

FIGURA 3.35. DIAGRAMA DE COMUNICACIÓN ENTRE DISPOSITIVO DE TELEMEDIDA Y GATEWAY ..................................8

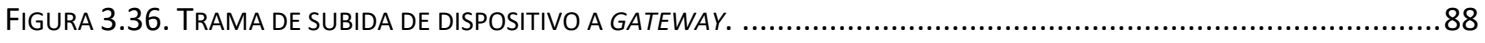

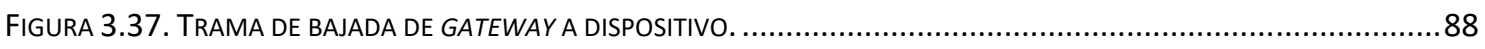

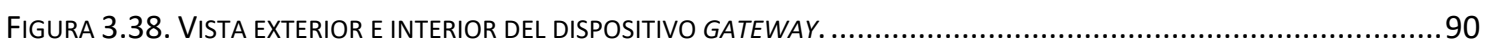

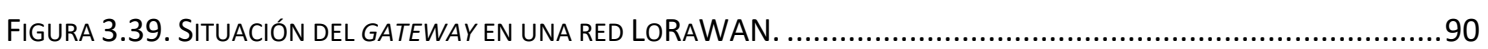

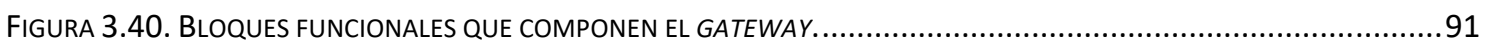

FIGURA 3.41. PLACA BASE RASPBERRY-PI, ADAPTADORA Y CONCENTRADOR LORAWAN........................................99

FIGURA 3.42. VISTA DEL CONJUNTO DE PLACAS Y DEL CONECTOR DE ANTENA UFL DEL CONCENTRADOR.........................99

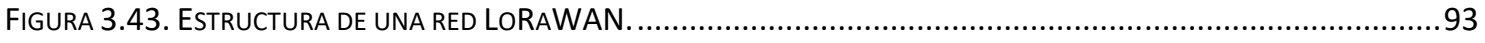

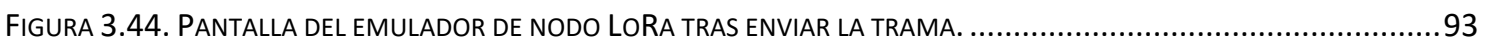

FIGURA 3.45. IMÁGENES DEL DISPOSITIVO DESARROLLADO PARA ESTUDIO DE COBERTURA...........................................94

FIGURA 3.46. DIAGRAMA DE BLOQUES DEL DISPOSITIVO PARA ESTUDIO DE COBERTURA............................................95

FIGURA 3.47. ESQUEMÁTICO ELÉCTRICO DEL DISPOSITIVO PARA ESTUDIO DE COBERTURA...........................................96

FIGURA 3.48. PLACA DE CIRCUITO IMPRESO DISEÑADA PARA EL DISPOSITIVO PARA ESTUDIO DE COBERTURA.....................97

FIGURA 3.49. IMAGEN DEL DISPOSITIVO INSTALADO EN VEHÍCULO DURANTE SESIÓN DE PRUEBAS.................................97

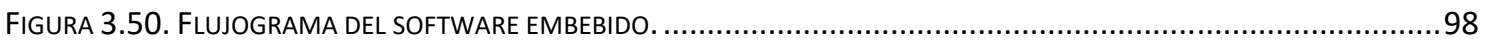

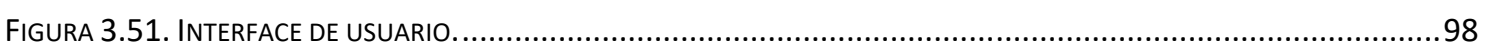

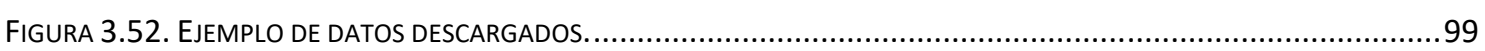

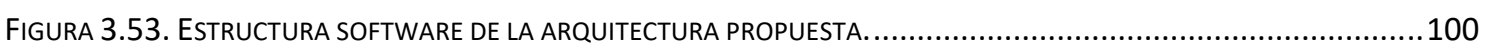

FIGURA 3.54. ORGANIGRAMA DE LAS DIFERENTES ENTIDADES DEL SERVIDOR LORAWAN. .......................................102

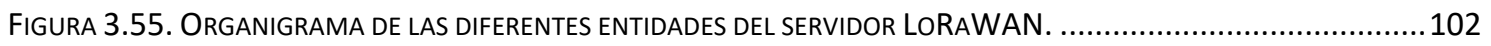

FIGURA 3.56. DECLARACIÓN DE GATEWAYS EN EL SERVIDOR LORAWAN.....................................................103

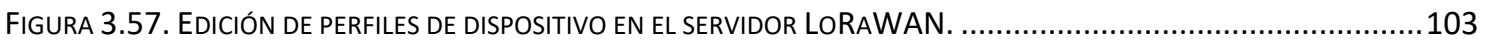

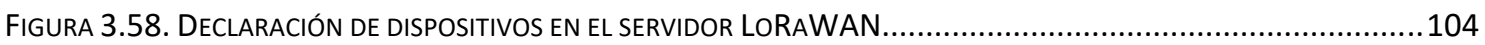

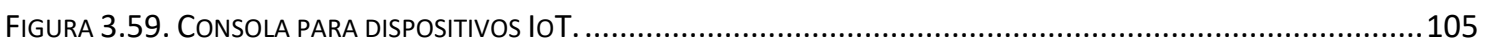

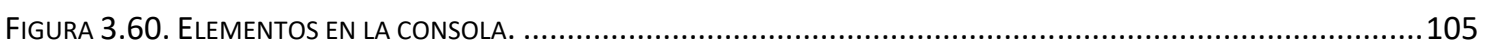

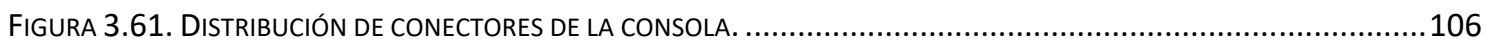

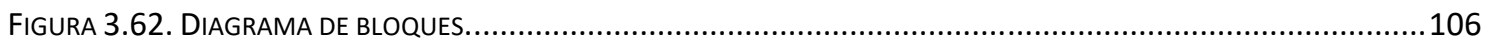

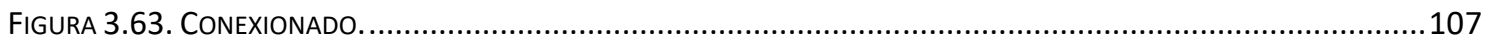

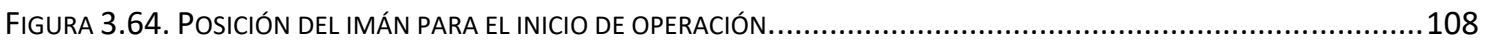

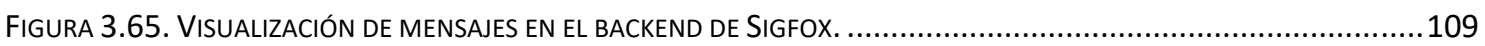

FIGURA 3.66. VISTAS EXTERIOR E INTERIOR DEL DISPOSITIVO. ..................................................................... 110

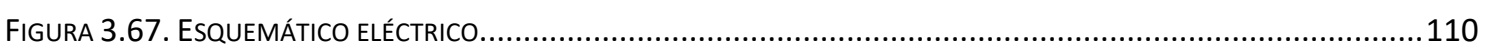

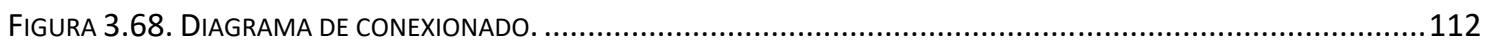

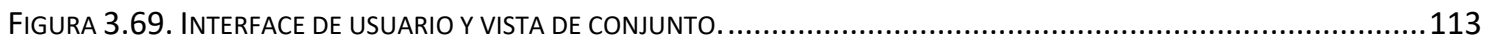

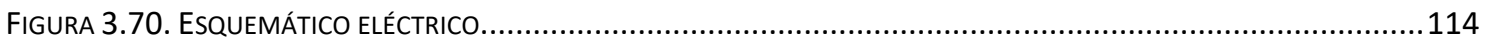

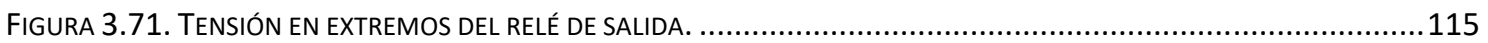




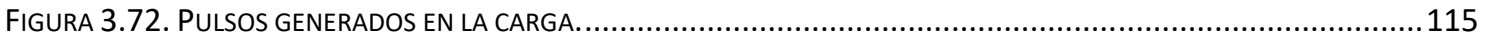

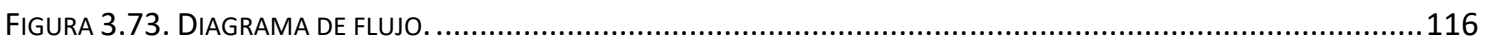

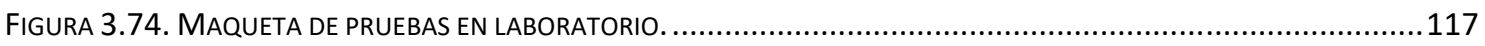

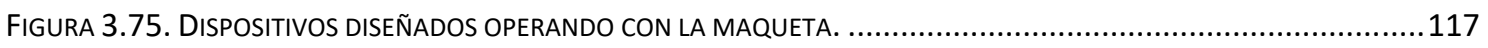

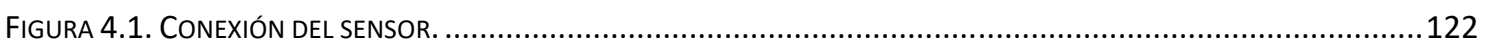

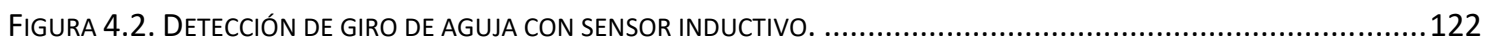

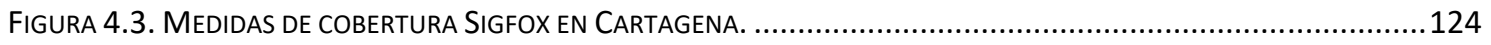

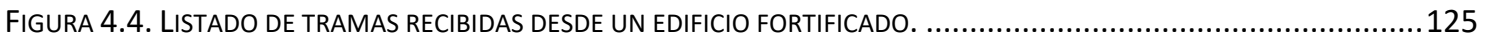

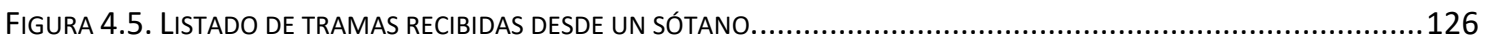

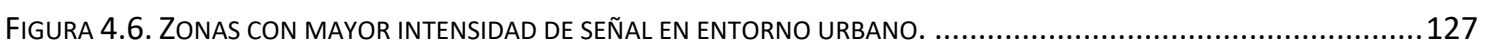

FIGURA 4.7. UBICACIÓN DE ESTACIONES BASE SIGFOX EN ENTORNO URBANO .................................................. 128

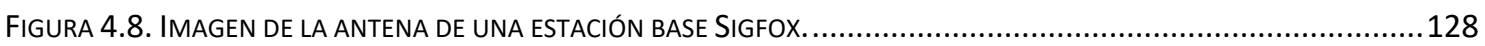

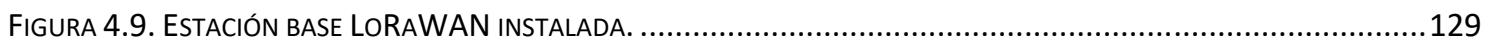

FIGURA 4.10. MEDIDAS DE COBERTURA LoRAWAN EN CARTAGENA............................................................. 130

FIGURA 4.11. ENTORNO DE PRUEBAS CON MAQUETA Y DISPOSITIVOS DESARROLLADOS..........................................131

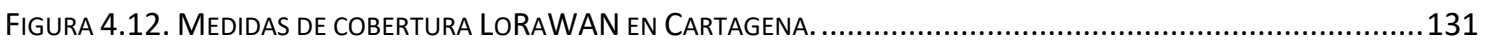

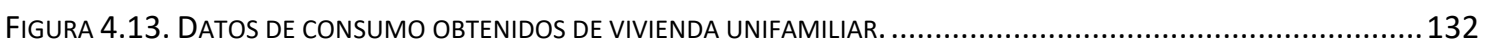

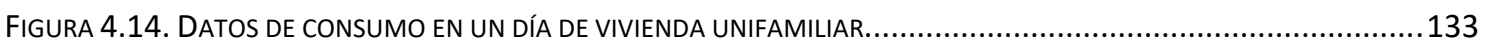

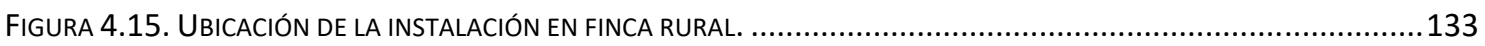

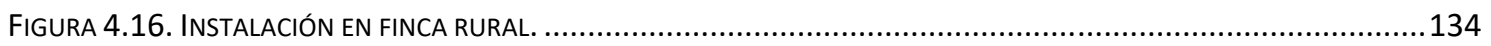

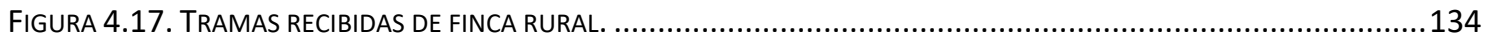

FIGURA 4.18. MUESTRAS PROCEDENTES DE 78 CONTADORES SELECCIONADOS AL AZAR........................................136

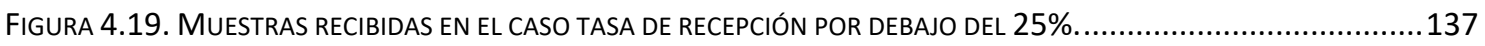

FIGURA 4.20. NÚMERO DE MUESTRAS RECIBIDAS CON TASA DE RECEPCIÓN ENTRE 25\% Y 75\%.................................138

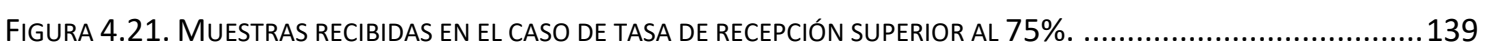

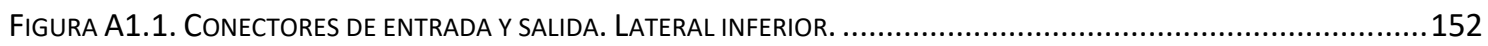

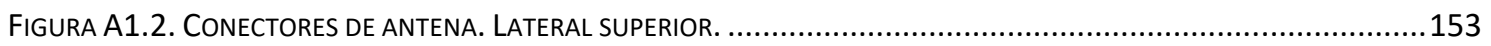

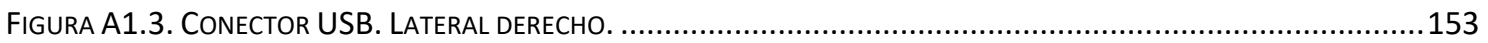

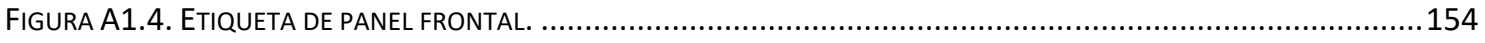

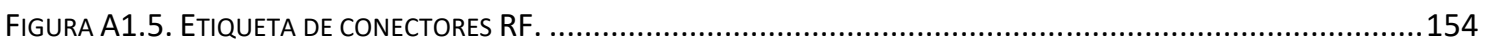

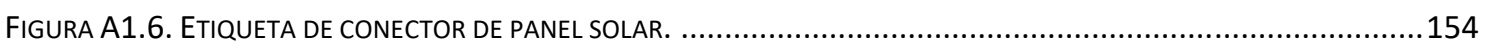

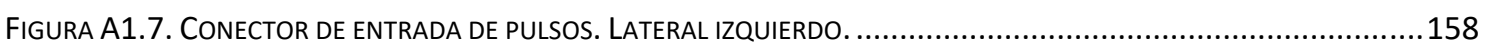

FIGURA A1.8. ConeCTOR DE SALIDA PARA ELECTROVÁLVULA. LATERAL DERECHO. ...................................................158

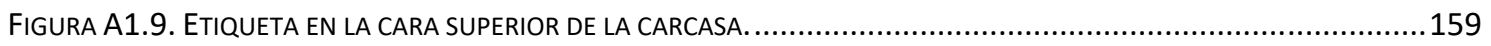

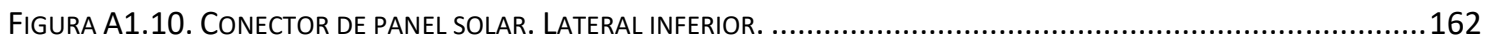

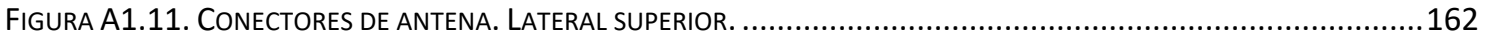

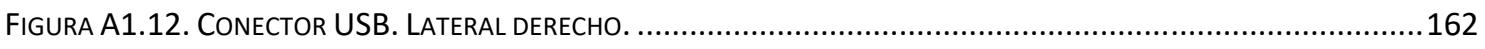

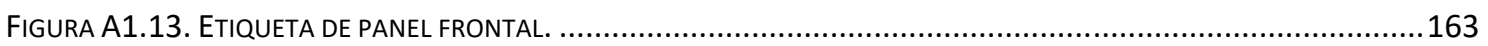

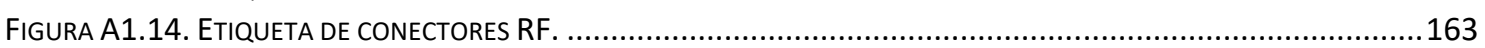

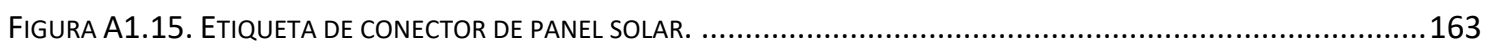

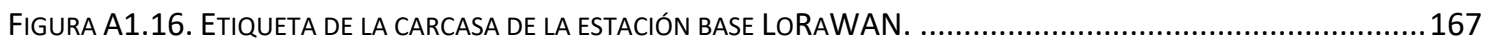

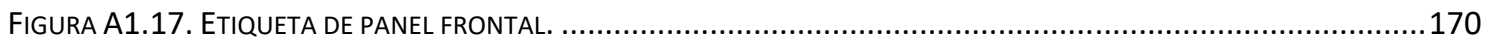

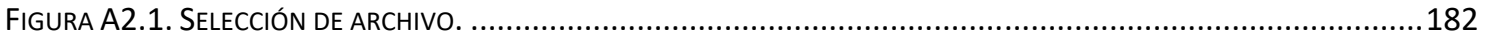

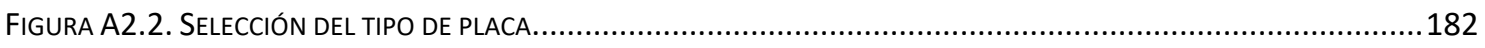

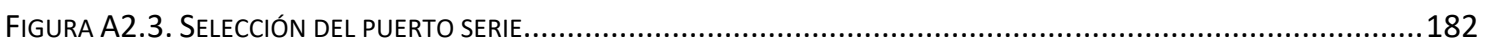

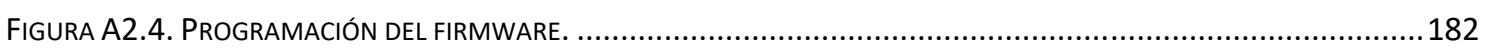

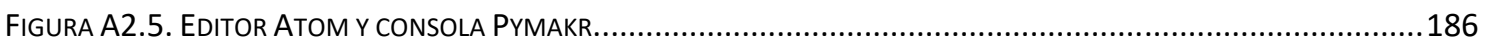





\section{ÍNDICE DETABLAS}

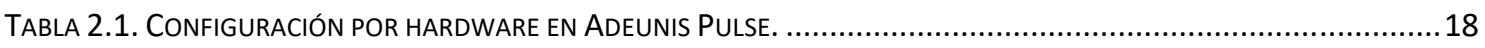

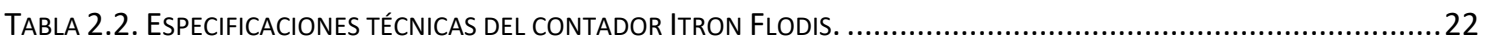

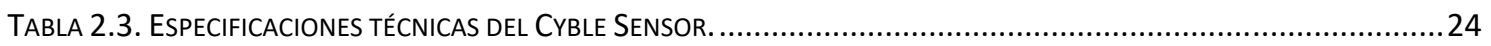

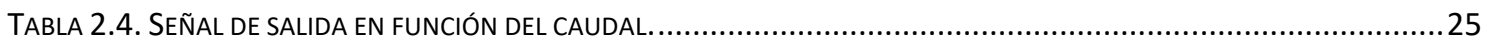

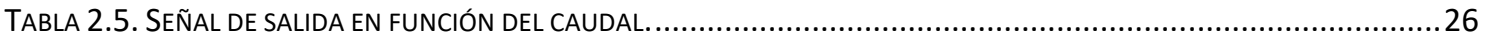

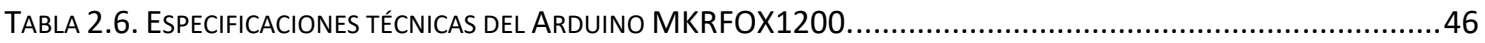

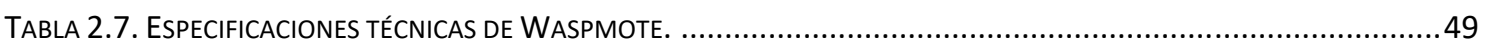

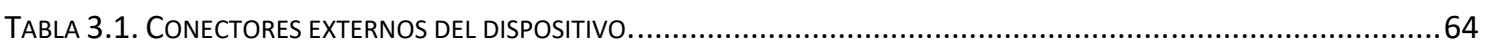

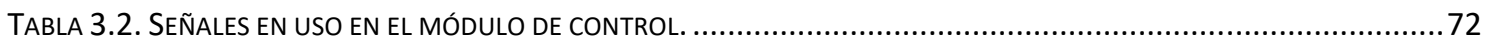

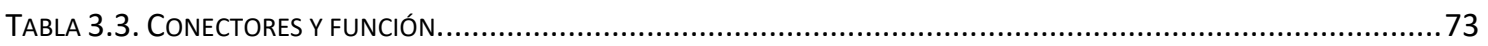

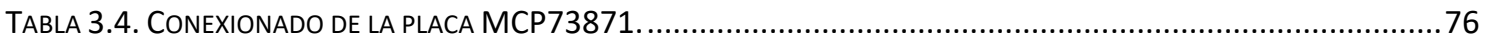

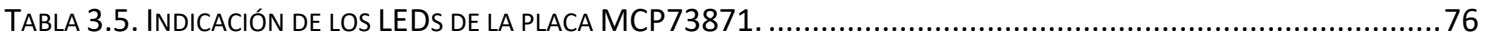

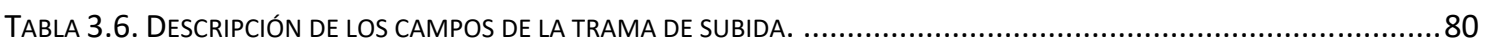

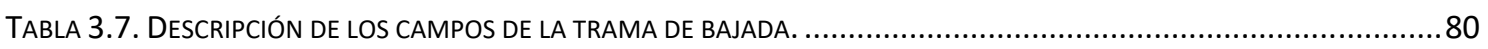

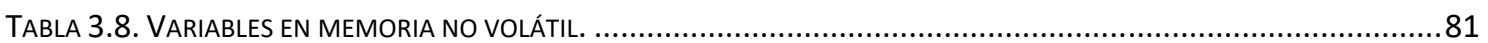

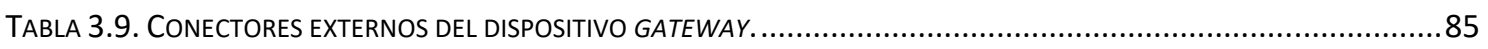

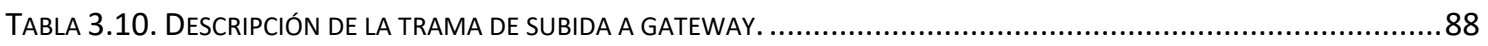

TABLA 3.11. POSIBILIDADES DE CONFIGURACIÓN MEDIANTE LOS MICRO-INTERRUPTORES.........................................107

TABLA 3.12. SELECCIÓN PARA ESTA APLICACIÓN DE LOS MICRO-INTERRUPTORES DE CONFIGURACIÓN. ............................107

TABLA 3.13. VALORES MEDIDOS DE LOS DIFERENTES PARÁMETROS ELÉCTRICOS...............................................111

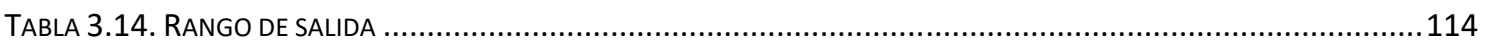

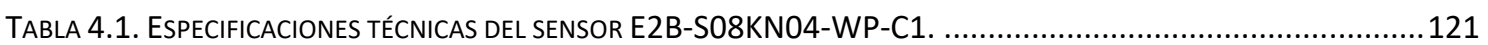

TABLA 4.2. RELACIÓN ENTRE NIVELES DE SEÑAL Y COLOR DE MARCADORES. ....................................................... 124

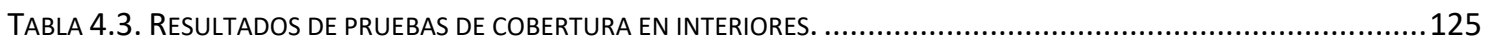

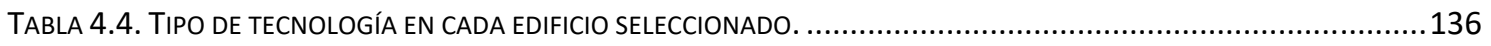

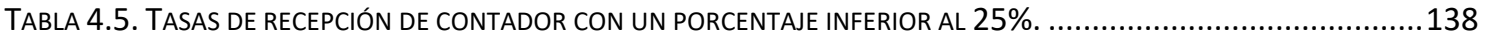

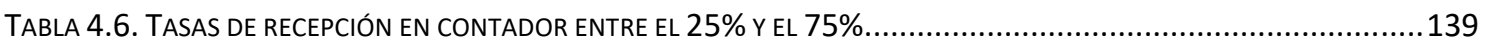

TABLA 4.7. TASAS DE RECEPCIÓN EN CONTADOR CON PORCENTAJE SUPERIOR AL 75\%..............................................140

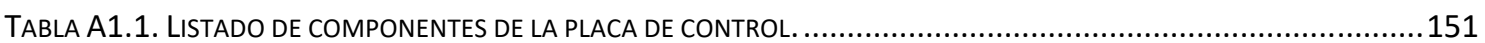

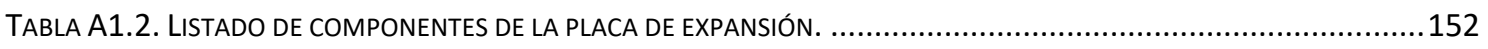

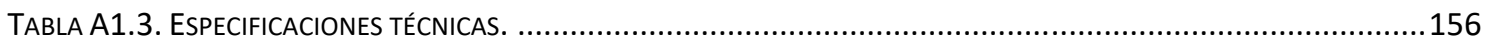

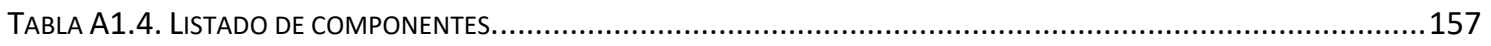

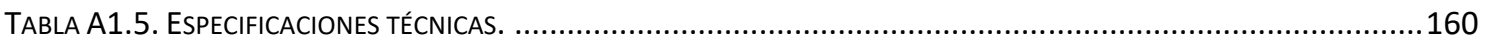

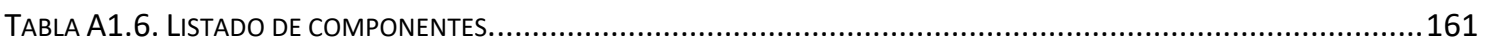

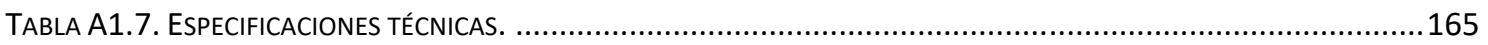

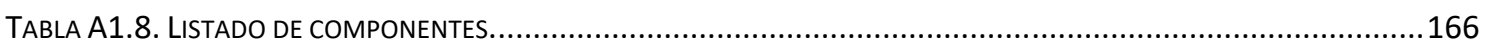

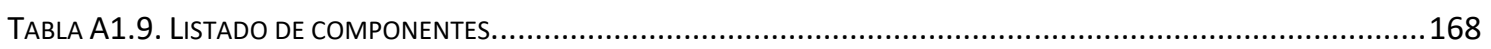

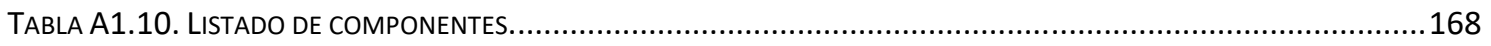

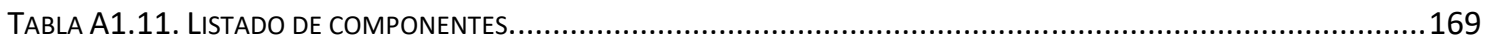

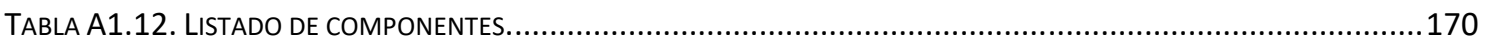

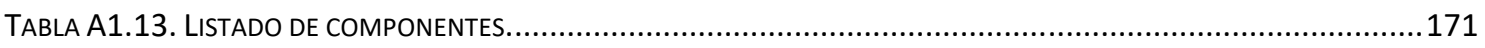

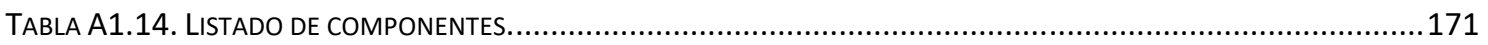

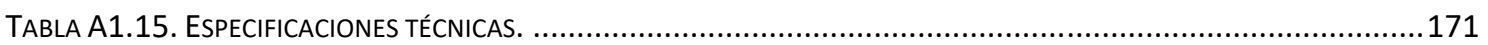

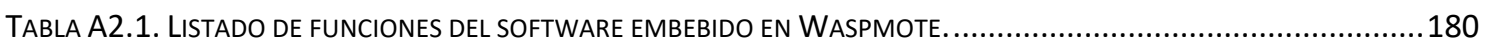

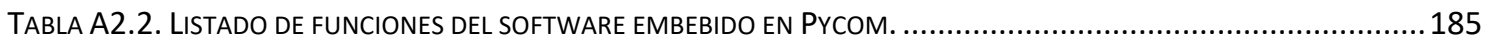



CAPÍTULO1

INTRODUCCIÓN 



\section{Introducción}

Internet of Things (IOT) o Internet de las Cosas está cobrando importancia y adquiriendo una participación cada vez mayor en todo ámbito a nivel mundial. Esta tendencia no aplica solo a personas en su vida cotidiana, sino que se convierte también en un factor determinante para las empresas. Lo que hace unos años era una tecnología potencial para habilitar nuevas iniciativas de negocio y mejoras operacionales, hoy en día ha pasado a la fase de implementación.

IoT crea un ecosistema [Rahman et al., 2016] que posibilita a las organizaciones reinventar la forma en la que se relacionan con sus clientes, proveedores, usuarios finales y otros interesados; en cómo funcionan sus procesos industriales y sus tiempos, además de ofrecer información que permitirá a las empresas irrumpir en el mercado con nuevos productos y/o servicios. Esto permite, entre otras aplicaciones, una atención y monitorización remotos para medir comportamientos y respuestas mucho más rápidas a situaciones de emergencia; prevención en el manejo de equipamiento, cadenas de suministro que entregan información en tiempo real de la oferta, demanda y envío a los clientes; y, en general, automatización y gestión de todos los activos a lo largo de su ciclo de vida [Lee y Lee, 2015].

El Internet de las cosas es el componente tecnológico fundamental sobre el que sienta sus bases el paradigma de los objetos conectados [ITU, 2005]. Cualquier objeto inteligente es capaz de enviar y recibir información a través de Internet aumentando con ello su funcionalidad 0 añadiéndole valor a la que ya tiene. El número de dispositivos finales del Internet de las cosas crecerá a un ritmo anual del 32.9\% (CAGR) hasta 2020, alcanzando una base instalada de 20.4 billones de unidades. Con este crecimiento masivo en el número de nodos, llegarán importantes avances tecnológicos en la tecnología de sensores, dispositivos, gateway y tecnologías de comunicación. Esto se combina con el crecimiento de las tecnologías de la información de vanguardia, donde la informática y los análisis se acercan más al nodo final para permitir más casos de uso en tiempo real [Ganguli y Friedman, 2005].

En la actualidad existe una gran variedad de tecnologías inalámbricas como WiFi, Bluetooth, 2G/3G/4G, ZigBee y otras muchas que nos permiten desplegar una red loT. Sin embargo, según las necesidades del diseño, será más apropiado el uso de unas frente a otras ya que cada tecnología tiene sus propias características y se adaptan a diferentes aplicaciones. El alcance de la red, el consumo de energía de los dispositivos o el ancho de banda de las transmisiones son algunos de los factores a tener en cuenta [Yang et al., 2017].

La conexión de tal ingente cantidad de objetos prevista supone nuevos retos para las redes que les den soporte, debiendo tener una alta escalabilidad para absorber el crecimiento exponencial previsto y simplicidad en el registro de nuevos dispositivos. En este contexto, los objetos deberán tener un consumo óptimo para que éstos puedan ser instalados en diversos lugares sin necesidad de fuentes de alimentación externas. Por otra parte, es interesante que la señal transmitida por los nodos pueda recorrer grandes distancias sin atenuarse excesivamente. Asimismo el tráfico de red se basará en el envío de pequeños paquetes de información cada cierto tiempo por lo que no será necesario disponer de grandes anchos de banda. Tampoco serán parámetros críticos la velocidad de transmisión o la latencia de la red. Lo más importante, será simplemente que los datos lleguen, al margen de la velocidad con que lo hagan. Bajo estas premisas la tecnología de red que mejor se adapta a los requisitos descritos es LPWAN (Low Power Wide Area Network) [Davoli et al., 2018]. 
La denominación LPWAN incluye redes de área amplia y baja potencia específicas para IoT. Permiten abordar el despliegue de grandes cantidades de objetos conectados con un coste contenido en infraestructura. Ofrecen conectividad de largo alcance y en entornos de alta densidad a dispositivos económicos y operados por baterías, los cuales requieren transmitir reducidas cantidades de datos a intervalos regulares de tiempo durante una larga vida útil [Sanchez-Iborra y Cano, 2016].

En este trabajo nos apoyamos en dos de los sistemas más populares de comunicaciones LPWAN para redes IoT como son Sigfox y LoRa. LoRa de Semtech es similar en rendimiento a Sigfox, pero con un enfoque de espectro ensanchado (SS). Semtech se centra en la fabricación de chips tanto para sensores como para estaciones base, y su modelo de negocio se basa en permitir a las empresas o usuarios individuales desplegar sus propias redes. En el trabajo aquí presentado, este aspecto es muy interesante porque se facilita muchísimo las comunicaciones Machine To Machine (M2M) y el relay en zonas donde los dispositivos ven minimizada 0 anulada la cobertura por distinto motivos. El modelo de negocio es distinto a Sigfox. Las compañías de telefonía móvil que se han aliado para promover la tecnología LoRa, la comercializan directamente como operadores donde el loT se trata de un servicio más que ofrecen a sus clientes. Sigfox es el nombre de una startup francesa y también el nombre de la tecnología. Es una solución que busca la compatibilidad con muchos fabricantes de módulos que adoptan una posición neutra como Avnet, Telit, Texas Instrument, etc, y por ello hay más variedad de dispositivos compatibles con esa tecnología en el mercado y en general lo que se conoce como dispositivos Sigfox ready. SigFox actúa directamente en el mercado como un operador y puede hablar directamente con los clientes finales, lo que supone una oportunidad para los integradores y los especialistas en soluciones y servicios que adopten esta tecnología.

Además de conectar una gran cantidad de objetos, la variedad de ellos también es muy amplia, con muchas categorías IOT distintas que engloban dispositivos de baja potencia, coches inteligentes, wearables 0 smart-meters. Estos últimos recolectan caudales de agua, gas 0 electricidad. Otros sensores y dispositivos envían datos de una gran variedad de fuentes, tales como temperatura, luz, sonido, etc. El resultado de ser un término tan amplio, es que no encontraremos una arquitectura universal que cumpla con los requisitos y las necesidades de cada una de las categorías del IOT. Un ejemplo de arquitectura de referencia para contadores inteligentes puede ser consultado en [Lloret et al., 2016], la cual cubre múltiples aspectos como una arquitectura en la nube que permite monitorizar, gestionar, interactuar y procesar datos de los dispositivos IoT. Sin embargo, una arquitectura de referencia global debe además incluir un modelo de redes para comunicar dispositivos y contar con agentes y códigos para los propios dispositivos, así como los requerimientos de los productos que son capaces de soportar esta arquitectura de referencia [Fremantle, 2018].

La gestión del ciclo integral del agua urbana, desde que el agua se recoge y llega al grifo, hasta que se devuelve a la naturaleza y se reutiliza, se divide en tres fases: abastecimiento, saneamiento y reutilización. El abastecimiento abarca desde la captación del agua hasta que llega a las acometidas y contadores de los edificios. El saneamiento se encarga del agua ya utilizada, que sale de las casas y se devuelve a su cauce natural respetando el medio ambiente. La reutilización, que se lleva a cabo en algunos casos, aprovecha el agua para usos distintos al consumo humano como el riego de jardines, la agricultura o algunos usos industriales. A lo largo del ciclo existen agentes encargados de la tarificación, de asegurar la atención al cliente y de recopilar estadísticas y elaborar normativas [Stewart et al., 2010]. 
El agua es necesaria para el mantenimiento de la vida humana. A pesar de que el suministro parece abundante, el agua no es un recurso ilimitado, particularmente el agua potable necesaria para la supervivencia humana. Sin esfuerzos de conservación, este suministro vital de agua puede verse agotado. La conservación también conlleva beneficios económicos, ya que la energía y los medios materiales también se conservan como resultado directo de los esfuerzos de preservación del agua.

En este marco, se ha realizado este trabajo de tesis doctoral en el que se propone el diseño, implementación y evaluación de una solución especializada en el manejo de agua residencial mediante el desarrollo y conexión de dispositivos inteligentes de medición de consumo de agua integrados en una arquitectura IoT mixta LoRa-Sigfox. Esta solución nos va a permitir hacer una gestión eficiente de la red de distribución. A diferencia de la mayoría de los sistemas actuales, y haciendo uso de comunicación bidireccional, permitirá además de la telelectura, actuar a distancia sobre el equipo del cliente interrumpiendo el suministro en caso de incidencias. La telelectura inteligente proporciona un mayor volumen de datos sobre el agua consumida, lo que permite un ahorro en el consumo de agua contribuyendo al desarrollo de la directiva para el agua de la Unión Europea [Voulvoulis et al., 2017] y a un mejor desarrollo de las Smart Cities 0 ciudades inteligentes [Montginoul y Vestier, 2018].

El trabajo realizado en esta tesis queda enmarcado dentro del denominado proyecto Tellot, orientado a la optimización de las infraestructuras hidráulicas de abastecimiento con telelectura de contadores inteligente basa en internet de las cosas (Ref. 2I16SA000044), alineado con la estrategia de investigación e innovación para la especialización Inteligente de la Región de Murcia, RIS3MUR. Durante el desarrollo de la solución aquí propuesta se ha optado por desarrollar varios dispositivos propios con hardware y software embebido. Como se expondrá en el capítulo tres, los productos comerciales no disponían de la totalidad de la funcionalidad requerida y adicionalmente se han conseguido las siguientes ventajas: 1) tener capacidad de personalización acorde a las necesidades del proyecto, 2) escalabilidad al poder añadirle nueva funcionalidad y 3) economía al orientar el diseño en este sentido. Así mismo, al tratarse de un diseño abierto tanto de hardware como de software, facilita a la comunidad investigadora el crear sus propias soluciones, superando los inconvenientes del resto de productos comerciales cerrados.

El dispositivo de telelectura inteligente loT desarrollado, a diferencia de productos similares, no sólo realiza la función de telelectura, sino que también es capaz de llevar a cabo telecontrol sobre dispositivos adyacentes como es el caso de una electroválvula. Se ha utilizado el mínimo número de componentes posibles y de coste reducido, respondiendo a las necesidades de simplicidad y economía. El uso de un lenguaje de alto nivel interpretado para su programación facilita la personalización. Al respecto de la conectividad de red, visto que en determinadas localizaciones, especialmente en el interior de edificios o sótanos, se ha encontrado ausencia 0 inestabilidad en el enlace a la red Sigfox, se ha creado un dispositivo que ubicado en una posición intermedia con buena conectividad Sigfox, envía a la red los datos recibidos de múltiples unidades sin conexión directa. Para ello, en este segundo tramo se lleva a cabo un radio-enlace punto a punto basado en LoRa que por las propias características de esta tecnología, se beneficia de un gran alcance y alta inmunidad a interferencias. De esta manera evitamos la instalación de micro estaciones base Sigfox ahorrando importantes costes de infraestructura. Por último, para las zonas con cobertura LoRaWAN se ha procedido al montaje e instalación de estaciones base propias que hacen la función de gateway a IP y están basadas en hardware y software abierto. 


\section{Objetivos}

Este trabajo se centra en diseñar, desplegar y verificar el correcto funcionamiento de una arquitectura de red loT mixta.

Como estudio previo se ha propuesto:

- Realizar un estudio de las tecnologías de comunicación específicas para loT actualmente disponibles y sus capacidades.

- Verificar sobre el terreno la cobertura y capacidades de las soluciones de comunicación loT disponibles.

- Estudiar las opciones en forma de dispositivos comerciales y kits electrónicos para desarrollo de aplicaciones IoT disponibles en el mercado, llevar a cabo su puesta en marcha y comparar sus capacidades.

- Montar un entorno de pruebas en laboratorio que simule una instalación de abonado final y sea capaz de enviar la información de consumo a través de diferentes dispositivos loT

Al respecto de la arquitectura propuesta, se establecen como objetivos los siguientes:

- Utilizar tecnologías de conectividad específicas para loT.

- Ser capaz de admitir diversidad de dispositivos para telelectura con independencia de su tipo de conectividad IoT.

- Incluir dispositivos loT para telelectura capaces de conectar mediante diferentes tecnologías de comunicación loT y capaces de llevar a cabo tareas de telecontrol.

- Integrar en la misma red diferentes tecnologías de comunicación loT.

- Ofrecer una solución de coste contenido para aquellas ubicaciones de difícil cobertura. 


\section{Planificación}

Se ha distribuido este trabajo en las siguientes fases:

- Planificación. Se han establecido los objetivos a cumplir, organizado las actividades a realizar y determinado los requisitos necesarios para cada una de ellas.

- Estudio del estado del arte. En ella se ha realizado un estudio de los contadores de agua de uso residencial y las posibles soluciones para lectura automatizada de la información de consumo. Se ha efectuado un estudio de las soluciones comerciales actualmente disponibles para telelectura, sus capacidades y carencias. Se han analizado diferentes kits de desarrollo electrónico para comunicaciones IOT. Por último, se han llevado a cabo pruebas de cobertura sobre el terreno de las diferentes tecnologías IoT con objeto de verificar sus prestaciones.

- Desarrollo de la arquitectura propia Ha comprendido el diseño de una arquitectura loT mixta que engloba diferentes tecnologías de comunicación y que engloba tanto elementos hardware como software propios. Además de contemplar el empleo de hardware comercial disponible, con el fin de completar las prestaciones ofrecidas por estos, se ha llevado a cabo el diseño y desarrollo de varios dispositivos propios tanto para telelectura y telecontrol, como para algunas funciones de red. También se ha puesto en marcha un entorno de pruebas en laboratorio para su verificación. A su vez, se ha puesto en marcha una plataforma software capaz de gestionar los datos provenientes de diferentes tecnologías.

- Evaluación de la solución propuesta Ha supuesto la verificación en diferentes escenarios de las capacidades reales de las tecnologías implicadas, así como la activación y validación de la arquitectura propuesta y los diferentes elementos que la integran mediante un despliegue en un entorno urbano. Ha incluido también un análisis de los datos y evaluación de rendimiento.

- Difusión de resultados. Se ha llevado a cabo la elaboración de conclusiones y difusión de resultados en publicaciones científicas de reconocido prestigio nacional e internacional. 
La planificación temporal se muestra de forma gráfica en el siguiente cronograma.

\begin{tabular}{|c|l|l|l|l|l|l|l|l|l|l|l|l|l|}
\hline \multicolumn{2}{|l|}{ ACTIVIDAD / MES (AÑO 1) } & $\mathbf{1}$ & $\mathbf{2}$ & $\mathbf{3}$ & $\mathbf{4}$ & $\mathbf{5}$ & $\mathbf{6}$ & $\mathbf{7}$ & $\mathbf{8}$ & $\mathbf{9}$ & $\mathbf{1 0}$ & $\mathbf{1 1}$ & $\mathbf{1 2}$ \\
\hline 1 & Inicio & & & & & & & & & & & & \\
\hline 1.3 & Planificación & & & & & & & & & & & & \\
\hline 2 & Estudio del estado del arte & & & & & & & & & & & & \\
\hline 2.2 & $\begin{array}{l}\text { Soluciones para lectura } \\
\text { automática de consumo }\end{array}$ & & & & & & & & & & & & \\
\hline 2.3 & $\begin{array}{l}\text { Estudio de las tecnologías de } \\
\text { comunicación específicas para } \\
\text { loT }\end{array}$ & & & & & & & & & & & & \\
\hline 2.4 & $\begin{array}{l}\text { Ensayo de kits de desarrollo } \\
\text { para comunicaciones loT }\end{array}$ & & & & & & & & & & & & \\
\hline 2.5 & Pruebas de cobertura & & & & & & & & & & & & \\
\hline
\end{tabular}

\begin{tabular}{|c|l|c|c|c|c|c|c|c|c|c|c|c|c|}
\hline \multicolumn{2}{|c|}{ ACTIVIDAD / MES (AÑO 2) } & $\mathbf{1 3}$ & $\mathbf{1 4}$ & $\mathbf{1 5}$ & $\mathbf{1 6}$ & $\mathbf{1 7}$ & $\mathbf{1 8}$ & $\mathbf{1 9}$ & $\mathbf{2 0}$ & $\mathbf{2 1}$ & $\mathbf{2 2}$ & $\mathbf{2 3}$ & $\mathbf{2 4}$ \\
\hline 3 & $\begin{array}{l}\text { Desarrollo de arquitectura } \\
\text { propia }\end{array}$ & & & & & & & & & & & & \\
\hline 3.3 .1 & $\begin{array}{l}\text { Prototipo de telemedida y } \\
\text { telecontrol con Waspmote }\end{array}$ & & & & & & & & & & & & \\
\hline 3.3 .2 & $\begin{array}{l}\text { Prototipo de telemedida y } \\
\text { telecontrol con PyCom }\end{array}$ & & & & & & & & & & & & \\
\hline 3.3 .3 & Gateway LoRa-Sigfox & & & & & & & & & & & & \\
\hline 3.3 .4 & Estación base LoRaWAN & & & & & & & & & & & & \\
\hline 3.3 .5 & $\begin{array}{l}\text { Dispositivo para el estudio } \\
\text { de cobertura }\end{array}$ & & & & & & & & & & & & \\
\hline 3.4 & Implementación software & & & & & & & & & & & & \\
\hline 3.5 & $\begin{array}{l}\text { Entorno de validación en } \\
\text { laboratorio }\end{array}$ & & & & & & & & & & & & \\
\hline 3.6 & Difusión de resultados & & & & & & & & & & & & \\
\hline
\end{tabular}




\begin{tabular}{|c|l|c|c|c|c|c|c|c|c|c|c|c|c|}
\hline \multicolumn{2}{|c|}{ ACTIVIDAD / MES (AÑO 3) } & $\mathbf{2 5}$ & $\mathbf{2 6}$ & $\mathbf{2 7}$ & $\mathbf{2 8}$ & $\mathbf{2 9}$ & $\mathbf{3 0}$ & $\mathbf{3 1}$ & $\mathbf{3 2}$ & $\mathbf{3 3}$ & $\mathbf{3 4}$ & $\mathbf{3 5}$ & $\mathbf{3 6}$ \\
\hline 4 & $\begin{array}{l}\text { Evaluación de la solución } \\
\text { propuesta }\end{array}$ & & & & & & & & & & & & \\
\hline 4.4 & Estudio de cobertura Sigfox & & & & & & & & & & & & \\
\hline 4.5 & $\begin{array}{l}\text { Estudio de cobertura } \\
\text { LoRaWAN }\end{array}$ & & & & & & & & & & & \\
\hline 4.6 & $\begin{array}{l}\text { Obtención de datos en } \\
\text { vivienda }\end{array}$ & & & & & & & & & & & & \\
\hline 4.7 & Despliegue & & & & & & & & & & & & \\
\hline 4.8 & Análisis de datos & & & & & & & & & & & & \\
\hline 5 & Difusión de resultados & & & & & & & & & & & & \\
\hline
\end{tabular}




\section{Descripción de los capítulos del trabajo}

Este trabajo de tesis doctoral se estructura en un total de cinco capítulos y dos anexos con información adicional. A continuación se presenta un listado de los mismos con su correspondiente título y un breve resumen de la información contenida en ellos.

Capítulo 1: Introducción.

En este capítulo se describe el marco en el que se inicia este trabajo y los objetivos que se pretenden alcanzar con la realización de esta tesis incluyendo un cronograma temporal distribuido en meses. Además se incluye una breve descripción del contenido de cada uno de los capítulos.

Capítulo 2: Estado del arte.

En el segundo capítulo se realiza una descripción de las soluciones para lectura automatizada de contadores de agua, de las tecnologías de comunicación loT implicadas en este trabajo y de los kits de desarrollo electrónicos evaluados para el diseño de dispositivos propios.

Capítulo 3: Propuesta de arquitectura IoT mixta.

Este tercer capítulo se centra en la arquitectura de red mixta propuesta incluyendo los desarrollos hardware y software llevados a cabo. En la parte de desarrollos hardware se describen tanto dos versiones del dispositivo de telemedida y telecontrol, como componentes de red propios que son una estación base y un gateway para zonas de sombra. Así mismo se describe un dispositivo para el estudio y verificación de cobertura. Para finalizar se incluye el entorno de laboratorio creado para las pruebas iniciales.

Capítulo 4: Evaluación de la solución propuesta.

En el cuarto capítulo se describen las tareas llevadas a cabo con el fin de verificar la viabilidad de la arquitectura propuesta. Comprende la puesta en marcha, estudio de cobertura, despliegue en un entorno real urbano, obtención de datos y análisis de los mismos.

Capítulo 5: Conclusiones.

En este quinto apartado se hace una recapitulación de los resultados obtenidos en los anteriores capítulos y para finalizar se enumeran las conclusiones alcanzadas con el presente trabajo de tesis doctoral.

\section{Anexo 1}

El primer anexo incluye información adicional referente al hardware propio desarrollado y que puede ser de utilidad para la fabricación de nuevas unidades.

\section{Anexo 2}

El segundo anexo engloba la información adicional referente al software embebido desarrollado al efecto, así como la metodología para la programación del mismo en los dispositivos. 
CAPÍTULO 2

ESTADO DEL ARTE 



\subsection{Introducción}

Se lleva a cabo en este capítulo un estudio previo del estado de la tecnología en el ámbito de contadores de consumo de agua para uso residencial, su predisposición a la telelectura, así como de dispositivos comercialmente disponibles para la transmisión de la información utilizando redes loT.

Se analizan Sigfox y LoRaWAN como las tecnologías LPWAN que más atención están consiguiendo actualmente por parte de la industria y la comunidad investigadora, además de operar en bandas radio ISM (Industrial Scientific Medical).

Con objeto de desplegar una red propia se evalúan posibles soluciones disponibles en el mercado en forma de estación base radio que facilite el servicio en su área de cobertura. De la misma manera se considera una plataforma software para operar una infraestructura de red loT sin necesidad de desplegar servidores propios para la gestión de los dispositivos.

Se presentan también varias posibilidades en forma de kits de desarrollo electrónico con comunicaciones loT integradas, útiles para la evaluación de la tecnología y para la rápida puesta en marcha de soluciones basadas en esta tecnología.

\subsection{Soluciones para lectura automática de consumo residencial de agua}

Se distingue entre contadores tradicionales diseñados para su lectura visual únicamente y los preparados para que la información pueda ser extraída por medios automáticos. Dentro de estos, se encuentran los que sólo disponen de una conexión eléctrica a la que enlazar otros equipos y aquellos que ya incluyen la electrónica necesaria para establecer una comunicación inalámbrica con una ubicación remota y hacer llegar la información por si mismos.

\subsubsection{Tipología de contadores según su capacidad para telelectura}

\subsubsection{Contadores tradicionales}

Este tipo de medidores está diseñado para su lectura de forma visual no automatizada. Los hay de dos tipos, de agujas y de tambores numerados, tal y como puede verse en la Figura 2.1. En los de agujas, cada cifra del valor actual del contador se representa mediante una esfera numerada del 0 al 9 . Para tomar la lectura se multiplica cada valor de aguja por su factor asociado y se lleva a cabo el sumatorio. La máxima precisión en los de cinco agujas es de 0.1 $\mathrm{m}^{3}$.
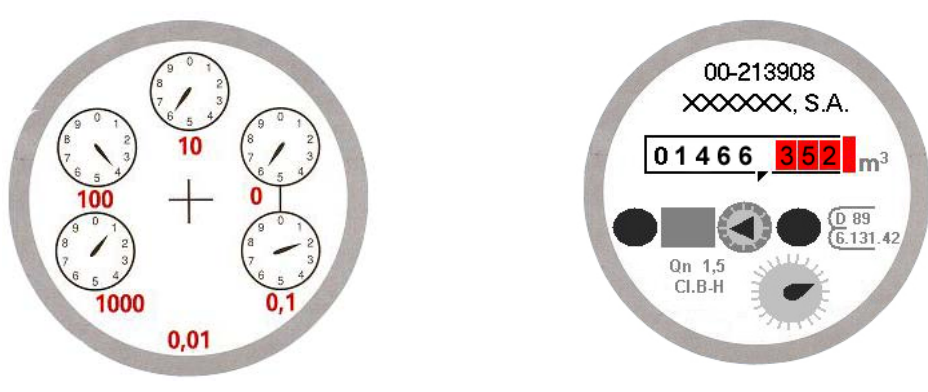

Figura 2.1. Contadores tradicionales de agujas y de tambores. 
En los de tambores, la lectura es directa. Cada tambor numerado representa una cifra del valor acumulado y se encuentran distribuidos de forma consecutiva y ordenada. Suele haber 8 cifras. Las cinco primeras sobre fondo blanco o negro indican los metros cúbicos. Las tres restantes sobre fondo rojo, indican los litros. La lectura suele tomarse en $\mathrm{m}^{3}$. Como elemento adicional se dispone de una aguja indicativa de litros. Viendo si la aguja tiene movimiento es fácil concluir si hay consumo de agua en la instalación. En algunos contadores, a pesar de estar fabricada la aguja en materia plástico, incluye un elemento metálico que puede estar imantado 0 no, para facilitar la lectura automatizada.

Por tanto, para llegar a cabo la lectura automatizada en estos dos tipos de contadores tradicionales, es necesario añadir un dispositivo adicional que extraiga información de los elementos mecánicos existentes, bien mediante la lectura de los tambores numéricos, bien detectando el giro de la aguja, y a partir de estos genere al menos, impulsos digitales. Estos impulsos ya pueden ser utilizados por un dispositivo externo para su transmisión o almacenaje de la misma manera que si el contador incorporase ya la salida.

Los posibles casos son:

- Lectura del valor numérico de los tambores.

Serían necesarias técnicas de visión artificial para la adquisición de una imagen del tambor y su interpretación en valores numéricos. Para evitar lecturas erróneas, la iluminación debe estar controlada, por lo que sería necesario acoplar sobre el contador una carcasa cerrada conteniendo la cámara y el sistema de iluminación artificial y que al mismo tiempo impidiera la entrada de luz exterior. Además del considerable tamaño del dispositivo, al quedar cubierta la zona del tambor, ya no sería posible una lectura directa de forma manual. También es a considerar el consumo energético necesario por la cámara y el sistema de iluminación.

- Detección del movimiento de la aguja.

- Caso de agujas plásticas:

En este caso es necesario remitirse al punto anterior y emplear otras técnicas para detectar el movimiento de la aguja tales como visión artificial o fotodiodos. Al tratarse de un elemento más simple que el tambor y no ser necesario detectar números sino sólo el paso de la aguja por una determinada posición de la esfera, podría utilizarse un sensor capaz de discernir entre el color de la aguja y el del fondo de la esfera.

- Caso de agujas con elemento metálico imantado:

Se puede detectar el paso de la aguja por una determinada región de la esfera, y por tanto su movimiento, mediante sensores de efecto hall o relés de tipo reed que detecten el campo magnético producido por el imán de la aguja. Este tipo de sistema podría ser susceptible a inferencias externas producidas por otros campos magnéticos.

- Caso de agujas con elemento metálico no imantado:

Este caso presenta inmunidad a campos magnéticos externos, sin embargo su detección es más compleja. Se puede utilizar un sensor inductivo o capacitivo con una distancia de detección acorde a la posición de la aguja en el interior del cuerpo del contador. Situando tres de estos sensores en diferentes posiciones de la esfera, es posible distinguir si el giro se produce en sentido horario o anti-horario, y con ello efectuar sólo 
en conteo al paso de la aguja en sentido correcto y evitar así como alertar de posibles casos de fraude.

\subsubsection{Contadores pre-equipados para lectura remota}

Se trata de dispositivos con cuerpo, instalación y presentación de la información similar a los medidores de consumo de agua tradicionales, pero con el añadido de incluir componentes pasivos para generar impulsos eléctricos cuando se detecta flujo de agua. Esto posibilita contabilizar el consumo efectuado en un periodo de tiempo y el tratamiento digital de dicha información para su almacenaje en otro equipo anexo, o la emisión inmediata o diferida a otras ubicaciones para proceder a la lectura remota.

Este tipo de medidores no incluye batería ni ninguna otra forma de alimentación interna 0 externa, por tanto, no requieren de mantenimiento periódico para la sustitución o recarga de baterías. Presentan un aspecto similar a los tradicionales mostrando el consumo acumulado en un panel donde cada cifra está formada por un indicador mecánico rotativo. Su principal diferencia con el contador tradicional es la presencia de un conector eléctrico o salida de un cable del cuerpo del contador con el otro extremo libre, como puede verse en la Figura 2.2.

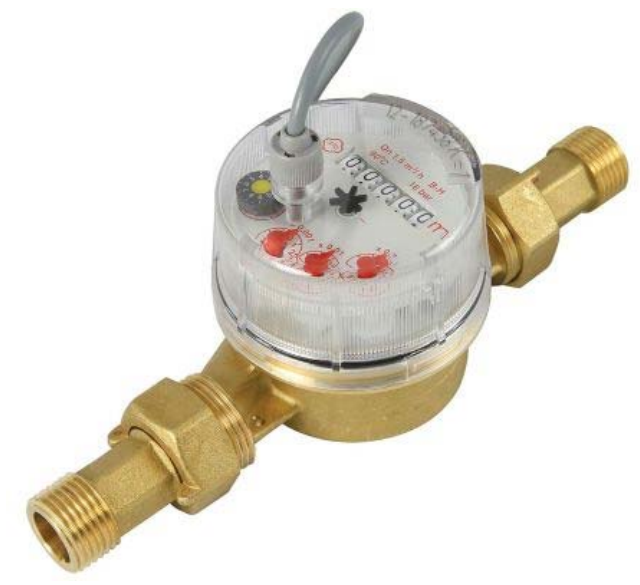

Figura 2.2. Contador de agua Altecnic GG-DALC25P1 con salida pulsada.

La detección de flujo de agua se realiza mediante dos elementos. Por una parte, un imán solidario al cuerpo de la turbina gira con el paso de líquido, y por otro, un relé de tipo reed cierra sus contactos al paso por sus proximidades del anterior imán. La activación del relé reed que presenta una muy baja resistencia a su salida, permite el paso de corriente y por tanto el cierre del circuito eléctrico al que conecte el contador. Si se trata de la entrada digital de un autómata programable, o un sistema digital embebido microprocesado, el flujo de agua puede ser detectado como una señal de impulsos cuya frecuencia es proporcional al caudal. Si los datos recogidos son almacenados en memoria, puede llevarse a cabo el conteo del consumo de agua en un periodo de tiempo para esa instalación.

\subsubsection{Contadores con comunicaciones integradas}

Este tipo de medidores incluye una fuente de energía interna, generalmente una batería primaria no recargable de Litio de tipo botón o de Litio Cloruro de Tionilo (Li-SOCl2). Suelen aprovechar la mayor disponibilidad energética para presentar la información de consumo en una pantalla de cristal líquido, y pueden llevar a cabo tareas adicionales como la detección de flujo de agua en sentido inverso a efectos de evitar fraude. 
Este tipo de medidores también puede incluir elementos de transmisión de la información a ubicaciones remotas mediante un emisor radio de ultra alta frecuencia incorporado, generalmente en banda ISM o libre de licencia siempre que se cumplan los requisitos para fines industriales, médicos o científicos. La tecnología de modulación y el protocolo empleados para la transmisión de la información pueden ser propietarios del fabricante, con lo que se requiere la adquisición de estaciones receptoras específicas para el mismo, o utilizar tecnología de comunicación diseñada para objetos conectados en el Internet de las cosas (IoT). Un ejemplo de esto son Sigfox y LoRa. Un ejemplo de contador con Sigfox puede verse en la Figura 2.3.

Aunque el consumo eléctrico es muy reducido y por tanto la autonomía de varios años, es necesario llevar a cabo mantenimiento periódico de la instalación para la sustitución de baterías del propio contador si la batería no es intercambiable.

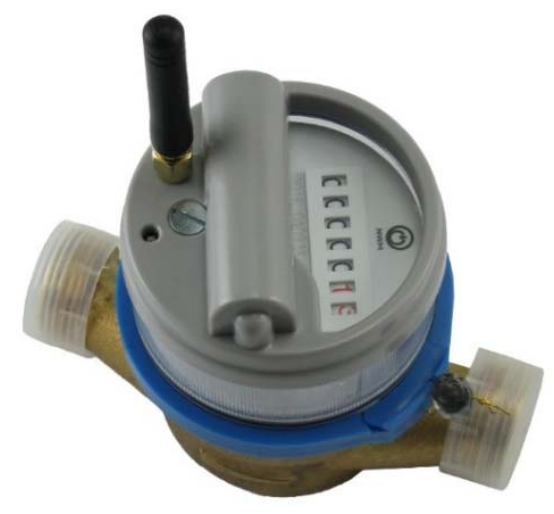

Figura 2.3. Contador de agua FL4-SG de NWM Europe con conectividad Sigfox.

\subsubsection{Contadores con capacidad de telemando}

Un reducido número de contadores con conectividad, utilizan un enlace radio bidireccional a través del cual envían la información de lectura y además pueden recibir órdenes para la actuación sobre una electroválvula externa que interrumpa o permita el suministro de agua a la instalación, como el mostrado en la Figura 2.4.

Algunos de estos productos constan de dos partes adquiribles de forma conjunta o por separado. Por un lado el contador digital con conectividad Sigfox para el envío periódico de la información de consumo, y opcionalmente, un módulo con electroválvula controlable de forma remota.

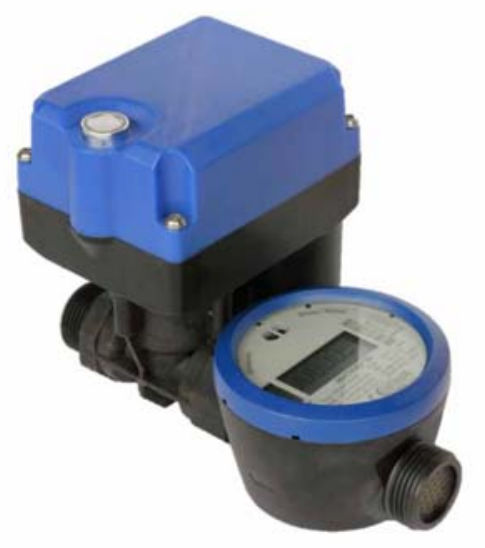

Figura 2.4. Contador con válvula Hydrokonekt Smart de Hydroko. 


\subsubsection{Dispositivos acoplables a contador tradicional para realizar telelectura}

\subsubsection{Sensor para lectura remota}

En el caso de contadores tradicionales sin salida de impulsos, es necesario acoplar un dispositivo externo capaz de generar estos a partir del movimiento de la aguja.

En ocasiones es el mismo fabricante del contador el que lo proporciona como opción, estando ya diseñado para un perfecto acoplamiento y sujeción al cuerpo del contador. Para algunos productos de este tipo es posible elegir diferentes referencias en función de su factor de desmultiplicación. De este modo, sólo se genera un pulso de salida al ocurrir un múltiplo de revoluciones de la aguja $(k=1, k=10, k=100)$. También es posible elegir entre modelos que disponen de varias salidas con las que proporcionan simultáneamente pulsos directos y desmultiplicados, además de una señal indicativa de si el flujo de agua está ocurriendo en sentido directo o inverso, y una señal de alarma que se activa cuando se separa el convertidor del cuerpo del contador. Su diseño y acoplamiento no impiden la lectura visual de las cifras.

La salida de este generador de pulsos puede conectarse a otro equipo externo como un registrador para almacenar la información o un transmisor para enviarla a una ubicación remota. Incluyen una batería interna no reemplazable y no recargable, con lo que al final de su vida útil será necesario reemplazar el producto.

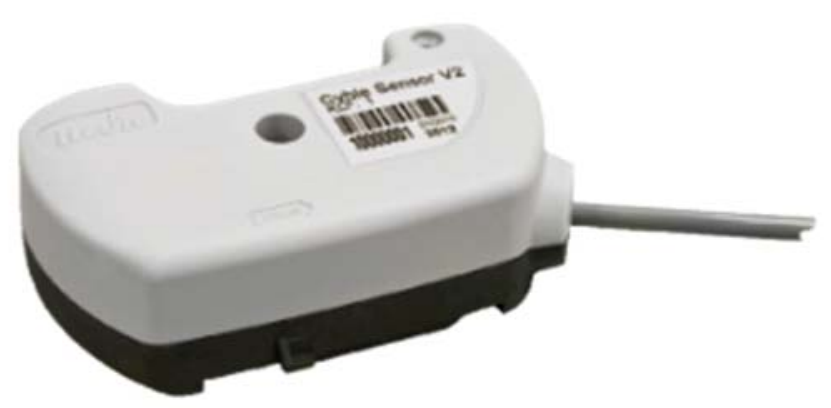

Figura 2.5. Generador de pulsos.

\subsubsection{Transmisor de consumo por redes loT}

Estos dispositivos recogen la señal producida por un generador de pulsos y son capaces de transmitir periódicamente la información de consumo de uno o varios contadores a través de redes IOT. Un mismo fabricante puede tener productos para conectividad a Sigfox o a LoRa. La alimentación proviene de una batería interna no recargable que le proporciona una autonomía de hasta 10 años en función de la configuración del intervalo de envío de datos.

Un ejemplo son los productos de Adeunis, el modelo ARF8048PA para Sigfox y el ARF8046PA para LoRaWAN, los cuales pueden verse en la Figura 2.6. Disponen de dos entradas para contadores e incluso una posible señal para alarma de fraude en caso de manipulación de la instalación. 


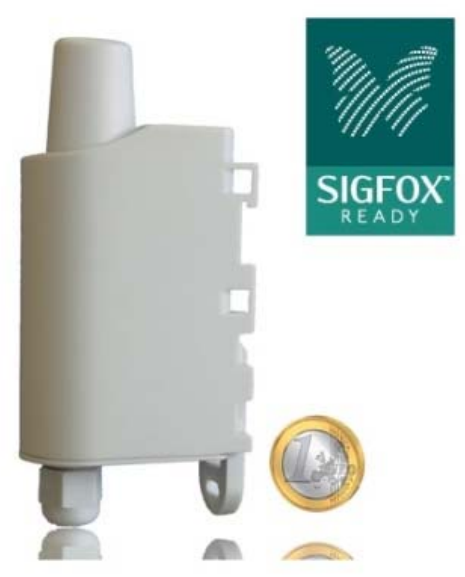

Figura 2.6. Transmisor de impulsos de Adeunis.

Se trata de dispositivos que operan en un solo tipo de red IoT, debiendo seleccionar desde el primer momento el modelo a adquirir. La alimentación proviene de una batería no recargable de tipo Litio Cloruro de Tionilo soldada en el circuito impreso, por lo que una vez finalizada su vida útil, el dispositivo ha de ser desechado o enviado a un servicio técnico especializado que efectue la operación de reemplazo de batería. Por esta razón, para evitar su consumo antes de iniciar la operación, el dispositivo debe activarse con el uso de un imán. A partir de ese momento no puede ser detenido.

La configuración inicial se lleva a cabo mediante microinterruptores en la placa de circuito. Debe seleccionarse su configuración antes de iniciar la operación con el imán, puesto que después cualquier cambio en ellos no tendrá efecto para evitar manipulaciones indeseadas. Puede seleccionarse el tipo de pulso de entrada, la unidad de consumo que representa cada pulso y la frecuencia de transmisión de datos. En la Tabla 2.1 pueden verse las posibilidades de configuración mediante interruptores. Esta parametrización puede ser modificada posteriormente mediante mensajes de configuración en el enlace descendente.

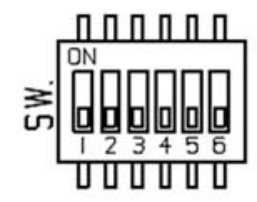

\begin{tabular}{|c|c|c|c|c|c|c|c|}
\hline Parameter & Value & SW1 & SW2 & SW3 & SW4 & SW5 & SW6 \\
\hline \multirow{4}{*}{ Meter type } & Electricity & $\mathrm{ON}$ & OFF & & & & \\
\hline & Water & OfF & OFF & & & & \\
\hline & Gas & OFF & $\mathrm{ON}$ & & & & \\
\hline & Heat & ON & ON & & & & \\
\hline \multirow{4}{*}{ Pulse weight } & $1 \mathrm{~L} / 10 \mathrm{dm}^{3} / 1 \mathrm{Wh}$ & & & OFF & OFF & & \\
\hline & $10 \mathrm{~L} / 10 \mathrm{dm}^{3} / 10 \mathrm{Wh}$ & & & OFF & $\mathrm{ON}$ & & \\
\hline & $100 \mathrm{~L} / 100 \mathrm{dm}^{3} / 100 \mathrm{Wh}$ & & & $\mathrm{ON}$ & OFF & & \\
\hline & $1000 \mathrm{~L} / 1 \mathrm{~m}^{3} / 1000 \mathrm{Wh}$ & & & ON & $\mathrm{ON}$ & & \\
\hline \multirow{4}{*}{ Transmission frequency } & 2 frames per day & & & & & OFF & OFF \\
\hline & 50 frames per day & & & & & OFF & $\mathrm{ON}$ \\
\hline & 100 frames per day & & & & & $\mathrm{ON}$ & OFF \\
\hline & 140 frames per day & & & & & ON & $\mathrm{ON}$ \\
\hline
\end{tabular}

Tabla 2.1. Configuración por hardware en Adeunis Pulse. 
El formato de la trama enviada y la información contenida en ella se encuentra en la Figura 2.7.

\begin{tabular}{|c|c|c|c|c|c|c|c|c|c|c|c|}
\hline 0 & 1 & 2 & 3 & 4 & 5 & 6 & 7 & 8 & 9 & 10 & 11 \\
\hline Code & Status & \multicolumn{10}{|c|}{ PAYLOAD } \\
\hline $0 \times 00$ & Cf Status & \multicolumn{10}{|c|}{ RESERVED } \\
\hline $0 \times 02$ & Cf Status & $\begin{array}{c}\text { Channel } 1 \\
\text { Type }\end{array}$ & \multicolumn{4}{|c|}{$\begin{array}{l}\text { Index } 1 \text { (LSB First) } \\
\text { Expressed in } 1 \mathrm{~W} . \mathrm{h}, 0.1 \mathrm{~L} \text { or } 0.1 \mathrm{dm} 3\end{array}$} & $\begin{array}{c}\text { Channel } 2 \\
\text { Type }\end{array}$ & \multicolumn{4}{|c|}{$\begin{array}{l}\text { Index2 (LSB First) } \\
\text { Expressed in } 1 \mathrm{~W} . \mathrm{h}, 0.1 \mathrm{~L} \text { or } 0.1 \mathrm{dm} 3\end{array}$} \\
\hline $0 \times 03$ & Cf Status & $\begin{array}{l}\text { Device } \\
\text { Type }\end{array}$ & \multicolumn{2}{|c|}{ Transmit period } & $\begin{array}{l}\text { Channel } \\
\text { On/Off }\end{array}$ & $\begin{array}{l}\text { Channel } \\
1 \text { Type }\end{array}$ & $\begin{array}{c}\text { Channel } 2 \\
\text { Type }\end{array}$ & $\begin{array}{l}\text { Pulse } \\
\text { Input } \\
\text { type }\end{array}$ & $\begin{array}{l}\text { Memo } \\
\text { switch }\end{array}$ & $\mathrm{x}$ & $x$ \\
\hline $0 \times 04$ & Cf Status & \multicolumn{2}{|c|}{ Pulse weight 1} & \multicolumn{4}{|c|}{ Memo Index 1} & \multicolumn{4}{|c|}{$x$} \\
\hline $0 \times 05$ & Cf Status & \multicolumn{2}{|c|}{ Pulse weight 2} & \multicolumn{4}{|c|}{ Memo Index 2} & \multicolumn{4}{|c|}{$x$} \\
\hline
\end{tabular}

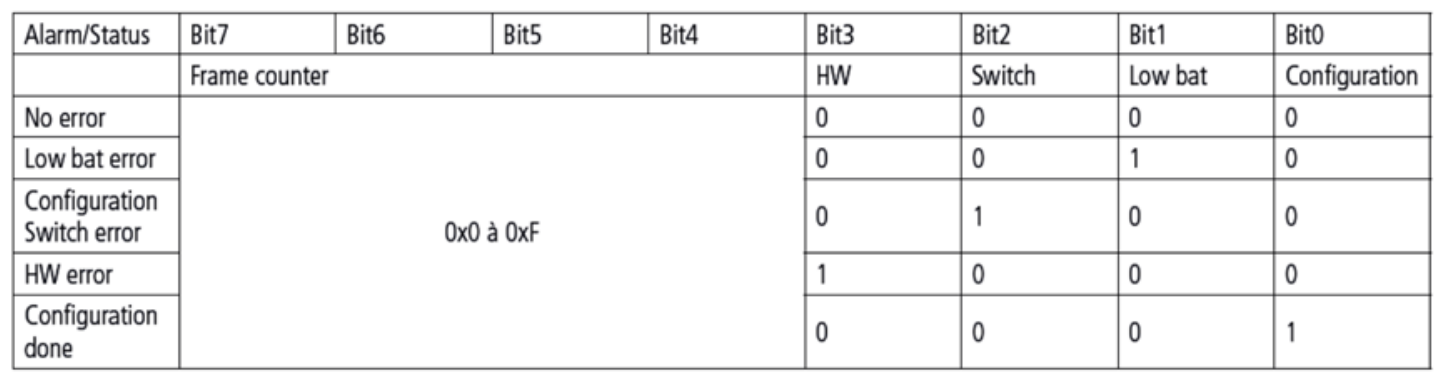

Figura 2.7. Trama enviada por Adeunis Pulse.

Un ejemplo de trama de tipo 2 con información de consumo y la correspondencia de cada campo es el siguiente:

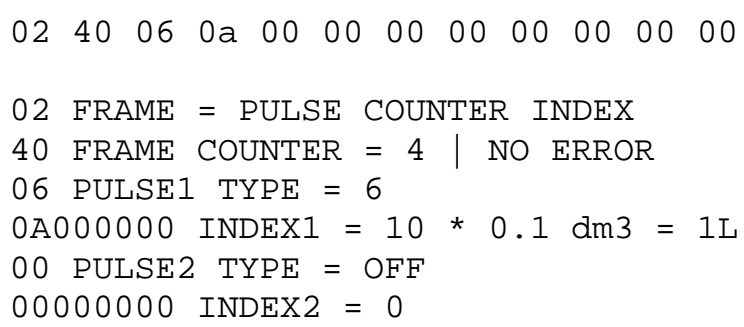

Cada 24 horas el dispositivo solicita a la red una trama de bajada con una posible nueva configuración. El formato de la trama de bajada y sus posibles campos pueden consultarse en la Figura 2.8. 
Payload downlink max $=8$ bytes, data format : Little endian, low significant byte first.

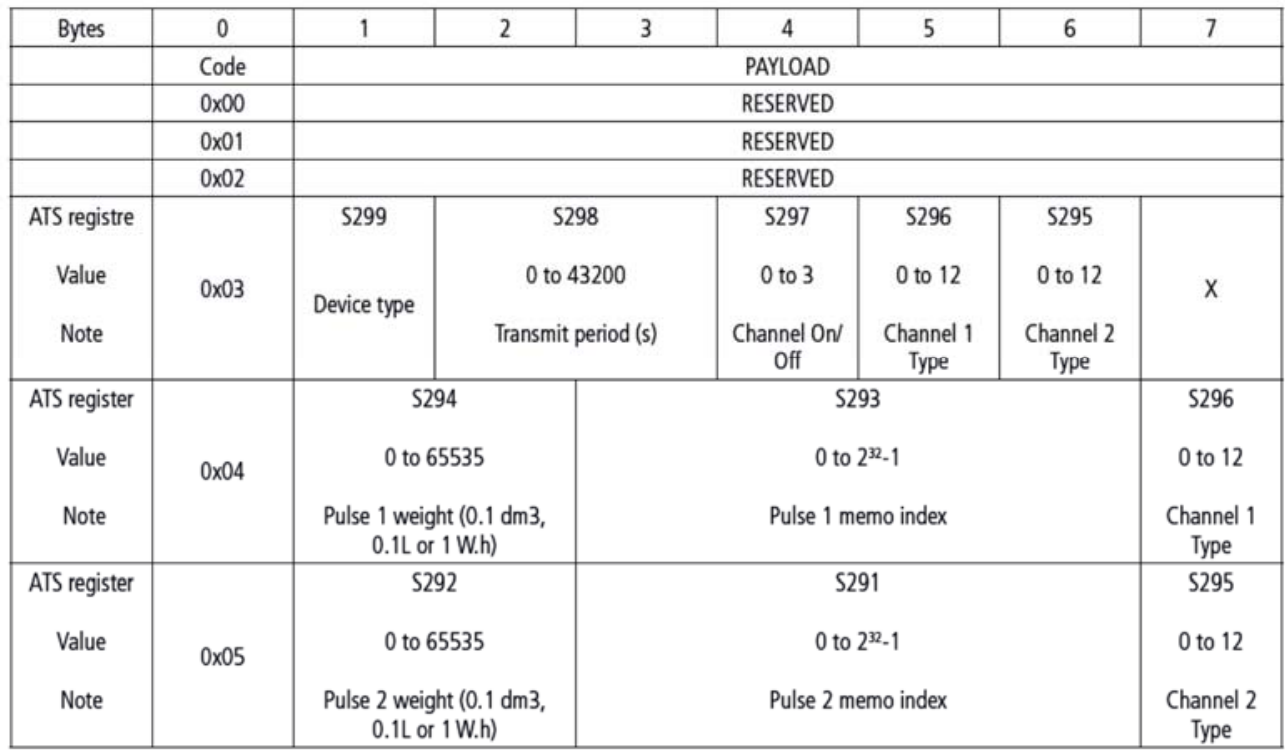

Figura 2.8. Trama de configuración para Adeunis Pulse.

Un ejemplo de trama de tipo 3 con nueva configuración enviada al dispositivo y la correspondencia de cada campo se muestra a continuación:

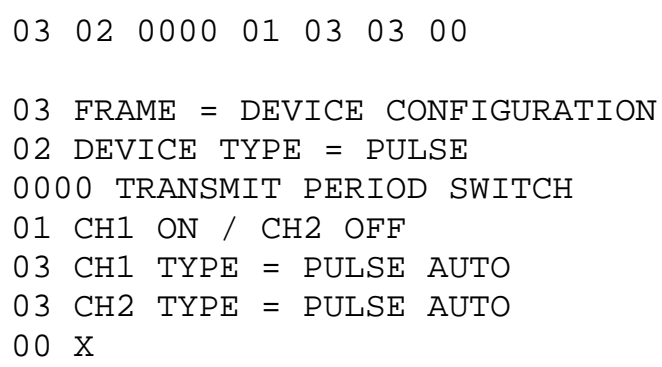

\subsubsection{Lector de información con transmisor integrado}

Estos dispositivos se acoplan a un contador tradicional y además de ser capaces de extraer la información de consumo, pueden enviarla utilizando redes de datos para objetos conectados. Debido a las diferentes posibilidades de acople y posición de los elementos móviles, han de estar diseñados para un modelo concreto de contador, generalmente por el fabricante del mismo. Ya que la tecnología de transmisión que utilizan permite un consumo energético reducido, incluyen una batería interna no reemplazable y no recargable, con lo que al final de su vida útil será necesario reemplazar el producto.

Un ejemplo es el Connit Live Blue Optical-A [Connit, 2018] para los contadores ARAD, que dispone de conectividad Sigfox y puede verse en la Figura 2.9. 


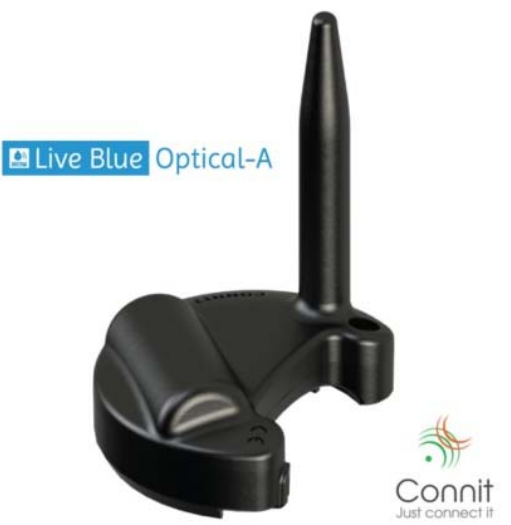

Figura 2.9. Connit Live Blue Optical-A para los contadores ARAD.

\subsubsection{Contador Itron Flodis}

El modelo de contador modelo Flosdis del fabricante Itron [Itron, 2018] es el mayoritariamente instalado en el núcleo urbano donde va a transcurrir este trabajo y por tanto ha sido seleccionado para verificar el funcionamiento del hardware desarrollado. Puede verse en la Figura 2.10.

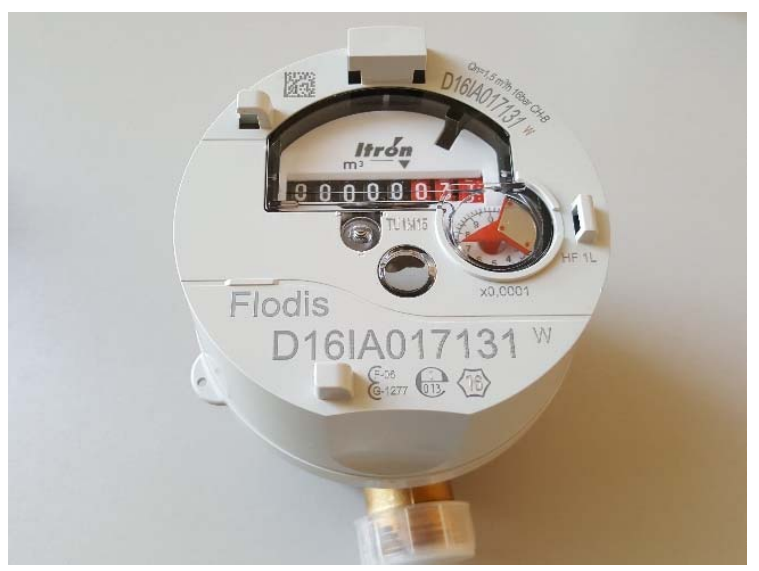

Figura 2.10. Contador Itron Flodis.

La lectura se realiza gracias a un tren de engranajes y un totalizador de 8 tambores, 5 de los cuales de fondo negro indican $\mathrm{m}^{3}$ y el resto en rojo proporcionan una precisión de 1 litro. Una aguja giratoria permite concretar hasta 0.1 litros y gracias a incluir una parte metálica no magnética, permite con un accesorio externo acoplable sin abrir el contador, detectar su movimiento y con ello llevar a cabo funciones de tele-lectura, análisis de caudales, gestión de dosificaciones 0 análisis de consumos entre otras. La carcasa plástica superior con el totalizador gira y es orientable para facilitar la lectura según la posición en que se haya instalado el contador. Posee un sistema de anti-empañamiento y es la denominada zona seca ya que se encuentra aislada del flujo de agua por una transmisión magnética entre la turbina y el totalizador. La carcasa puede ser de termoplástico sellado o de vidrio-metal con más robustez y estanqueidad incluso en inmersión. Para contabilizar el consumo se utiliza una turbina con pivotaje, y es la única pieza en movimiento sumergida en agua. La fricción del conjunto está estudiada para garantizar durabilidad en el rango de caudales admisible. Utiliza tecnología de chorro único que con un inyector guía el chorro de agua hasta la turbina antes de accionarla. Al utilizar este método e incluir un filtro a la entrada, es poco sensible a partículas en suspensión en el agua que puedan afectar a su correcto funcionamiento en el tiempo. Están disponibles varias 
versiones según el caudal aceptado que afectan a las dimensiones del contador. Una vista interna de corte se encuentra en la Figura 2.11.

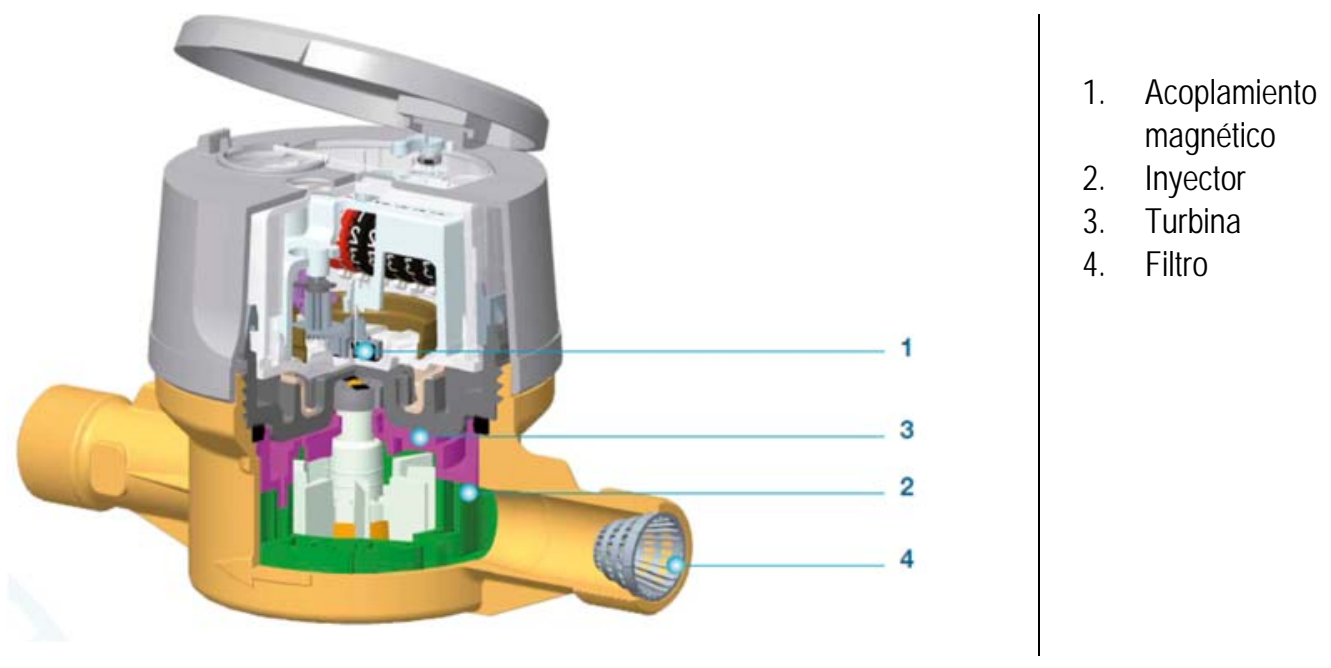

Figura 2.11. Elementos internos del contador Itron Flodis.

Sus características más destacadas son las siguientes:

- Apto para agua potable.

- De tipo chorro único con gran dinámica.

- Transmisión magnética.

- Totalizador seco.

- Fácil lectura.

- Preparado para tele-lectura.

- Cumple con MID, la directiva del consejo europeo sobre instrumentos de medida.

- Varias versiones según caudal nominal.

Las especificaciones técnicas pueden consultarse en la siguiente Tabla 2.2.

\begin{tabular}{l|c|c}
\hline \multicolumn{1}{c|}{ Característica } & Valor & Unidad \\
\hline Diámetro nominal DN & 15 & $\mathrm{~mm}$ \\
\hline Caudal mínimo Q1 & 12.5 & $\mathrm{I} / \mathrm{h}$ \\
\hline Caudal de transición Q2 & 20 & $\mathrm{I} / \mathrm{h}$ \\
\hline Caudal nominal Q3 & 2.5 & $\mathrm{~m}^{3} / \mathrm{h}$ \\
\hline Caudal máximo Q4 & 3.125 & $\mathrm{~m}^{3} / \mathrm{h}$ \\
\hline Presión máxima admisible & 16 & $\mathrm{bar}$ \\
\hline Rango dinámico Q3 / Q1 & 200 & - \\
\hline Caudal de arranque & 5 & $\mathrm{I} / \mathrm{h}$ \\
\hline Máxima lectura & $10^{\wedge} 5$ & $\mathrm{~m}^{3}$ \\
\hline Paso de lectura & 0.05 & $\mathrm{I}$ \\
\hline Temperatura de funcionamiento & $0.1-50$ & ${ }^{\circ} \mathrm{C}$ \\
\hline Máxima temperatura & 60 & ${ }^{\circ} \mathrm{C}$ \\
\hline Peso & 0.9 & $\mathrm{~kg}$ \\
\hline
\end{tabular}

Tabla 2.2. Especificaciones técnicas del contador Itron Flodis. 


\subsubsection{Generador de pulsos Itron Cyble}

El módulo opcional Cyble acoplable en la parte superior del contador Itron Flodis sin necesidad de abrir o desprecintar este, da la posibilidad de extraer la información de consumo y disponer de lectura remota para así simplificar los trámites necesarios para la facturación. Su salida puede ser utilizada por cualquier sistema de registro o transmisión remota de información sin necesidad de que sea propietario del mismo fabricante. Puede verse una imagen del mismo acoplado a un contador en la Figura 2.12.

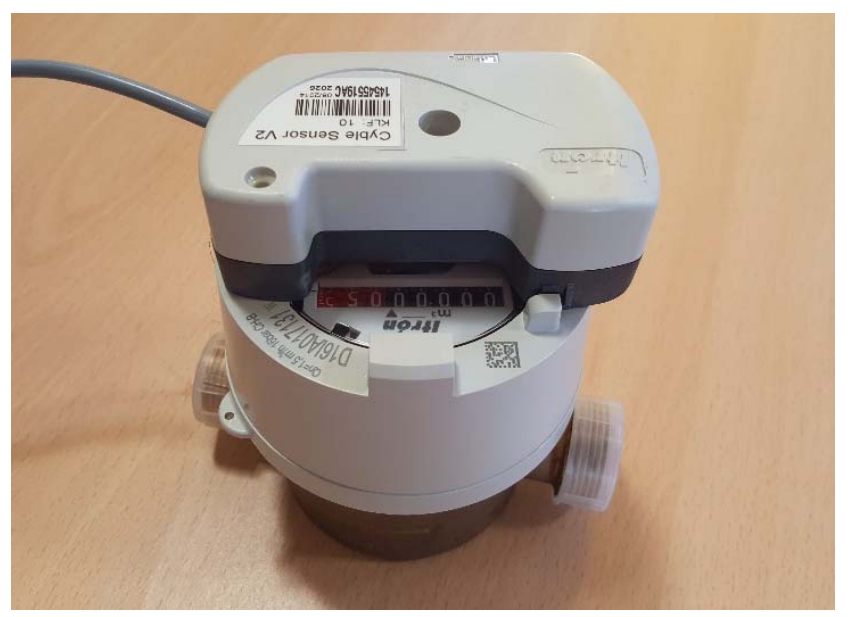

Figura 2.12. Contador con módulo Cyble Sensor.

Puede elegirse entre varias versiones según el factor de desmultiplicación y las señales de salida disponibles. El factor de desmultiplicación $\mathrm{K}$ define el número de revoluciones detectadas antes de generar un impulso de salida. En casos en los que no sea necesaria toda la resolución del contador, evita señales innecesarias en la entrada del dispositivo al que se conecta aumentando la autonomía de este en caso de despertarse al recibir cada impulso, o permitiendo que trabaje a una velocidad de adquisición inferior.

También es posible elegir entre una versión con una salida simple de 2 conductores libres de potencial y polaridad equivalente a un relé, o una versión con señales de salida adicionales. Estas incluyen además de los impulsos equivalentes a revoluciones de la aguja, una salida de impulsos desmultiplicada de tipo colector abierto y señales adicionales de dirección de flujo y fraude. Pueden verse las señales en la Figura 2.13.
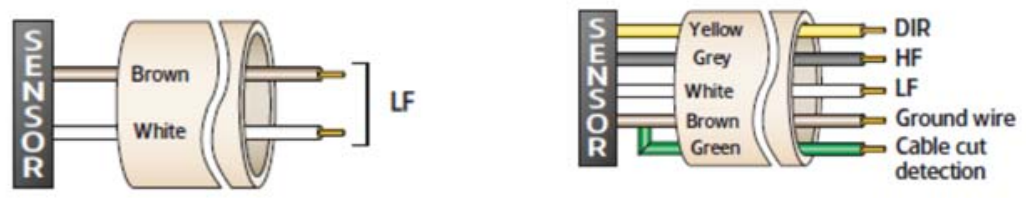

Figura 2.13. Señales disponibles en el Cyble Sensor.

La energía necesaria para su operación la obtiene de una batería interna de Litio, no siendo esta reemplazable, por lo que una vez concluida su vida útil estimada por el fabricante en 12 años, el dispositivo ha de ser sustituido.

Sus características más destacables son las siguientes: 
- Detección del paso de aguja por una posición, resolución 1 litro.

- Diferentes versiones según el factor de multiplicación K=1, 2.5, 10, 25, 100, 1000.

- Dos versiones según salida:

- 2 conductores,

- LF: Señal de baja frecuencia libre de potencial y sin polaridad.

- 5 conductores

- LF: Señal de baja frecuencia con polaridad y multiplicada. LF=HF*K

- CC: Alarma de manipulación. Señal conectada internamente a masa para detectar corte del cable.

- DIR: Indica un sentido inverso del caudal.

- HF: Señal de alta frecuencia sin desmultiplicar y con polaridad. Pulso por revolución de la aguja. Generada siempre independientemente de la dirección del caudal.

- Masa

- Aplicaciones por tipo de salida

- Salida baja frecuencia.

- Lectura remota

- Consumos acumulados

- Salida alta frecuencia.

- Análisis de caudales

- Conversión de frecuencia a corriente

- Control automático

- Inmune a posibles fraudes por cambios magnéticos

- Inmune a vibraciones

- Puede operar en pozos inundados y está diseñado para entornos adversos.

Sus especificaciones técnicas se muestran en la Tabla 2.3.

\begin{tabular}{lccc}
\hline \multicolumn{1}{c}{ Dato } & 2 hilos & 5 hilos & Unidades \\
\hline BF & SI & SI & - \\
\hline HF & NO & SI & - \\
\hline Cable cortado & NO & SI & - \\
\hline Dirección & $\mathrm{NO}$ & $\mathrm{SI}$ & - \\
\hline Batería integrada & Litio & Litio & - \\
\hline Autonomía & 12 & 12 & $\mathrm{Años}$ \\
\hline Tipo de salida & $\mathrm{DC}$ & $\mathrm{DC}$ & - \\
\hline Corriente máxima salida & 100 & 100 & $\mathrm{~mA}$ \\
\hline Tensión máxima salida & 30 & 30 & $\mathrm{~V}$ \\
\hline Potencia máxima salida & 1 & 1 & $\mathrm{~W}$ \\
\hline Polarización salida & $\mathrm{NO}$ & $\mathrm{SI}$ & - \\
\hline Tipo de salida & Open-colector & Open-colector & - \\
\hline Capacidad & 600 & 600 & $\mathrm{pF}$ \\
\hline Longitud del cable & 5 & 5 & $\mathrm{~m}$ \\
\hline Diámetro del conductor & 0.9 & 0.9 & $\mathrm{~mm}$ \\
\hline Dimensiones del cable & $6.6 \times 2.3$ & $6.6 \times 2.3$ & $\mathrm{~mm}$ \\
\hline Temperatura de operación & $-10 \mathrm{a}+55$ & $-10 \mathrm{a}+55$ & ${ }^{\circ} \mathrm{C}$ \\
\hline Temperatura de almacenamiento & $-20 \mathrm{a}+55$ & $-20 \mathrm{a}+55$ & ${ }^{\circ} \mathrm{C}$ \\
\hline Grado de protección & $\mathrm{IP} 68$ & $\mathrm{IP} 68$ & - \\
\hline
\end{tabular}

Tabla 2.3. Especificaciones técnicas del Cyble Sensor. 
Según el rango de trabajo del contador, la salida correspondiente del generador de impulsos es la mostrada en la Tabla 2.4.

\begin{tabular}{lcccccc}
\hline & $\begin{array}{c}\text { Caudal } \\
\mathrm{m}^{3} / \mathrm{h}\end{array}$ & $\begin{array}{c}\text { Caudal } \\
\mathrm{I} / \mathrm{h}\end{array}$ & $\begin{array}{c}\text { Caudal } \\
\mathrm{I} / \mathrm{min}\end{array}$ & pulsos $/ \mathrm{min}$ & pulsos $/ \mathbf{s}$ & $\begin{array}{c}\text { Intervalo } \\
(\mathbf{s})\end{array}$ \\
\hline Qn & 1.5 & 1500 & 25 & 25 & 0.416 & 2.4 \\
\hline Qmax & 3 & 3000 & 50 & 50 & 0.833 & 1.2 \\
\hline Qmin & 0.015 & 15 & 0.25 & 0.25 & 0.004 & 240 \\
\hline
\end{tabular}

Tabla 2.4. Señal de salida en función del caudal.

Dado que la salida del Cyble-Sensor es libre de potencial, para visualizar los pulsos en el osciloscopio se ha realizado el siguiente circuito eléctrico de prueba mostrado en la Figura 2.14.

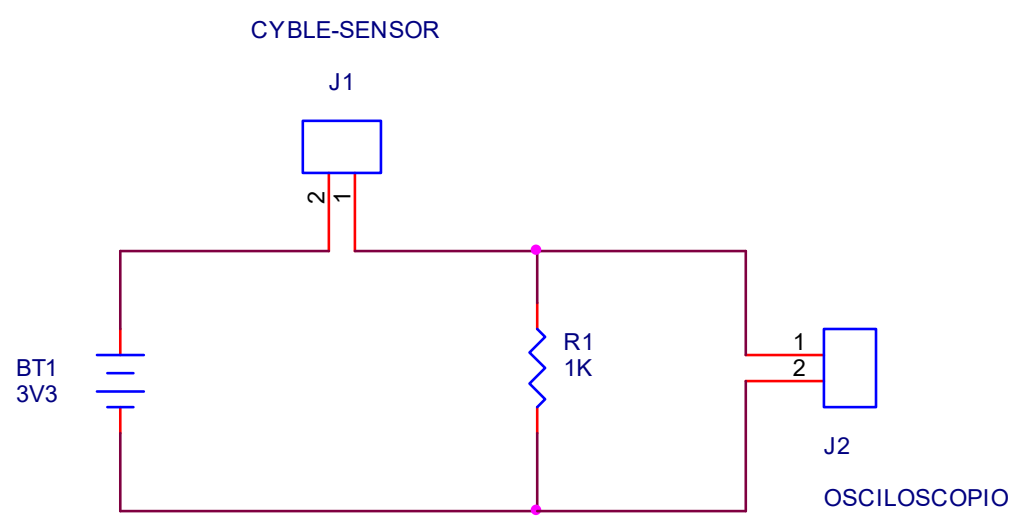

Figura 2.14. Circuito eléctrico de prueba del generador de impulsos.

La señal de pulsos a la salida visualizada en el osciloscopio se muestra en la Figura 2.15.

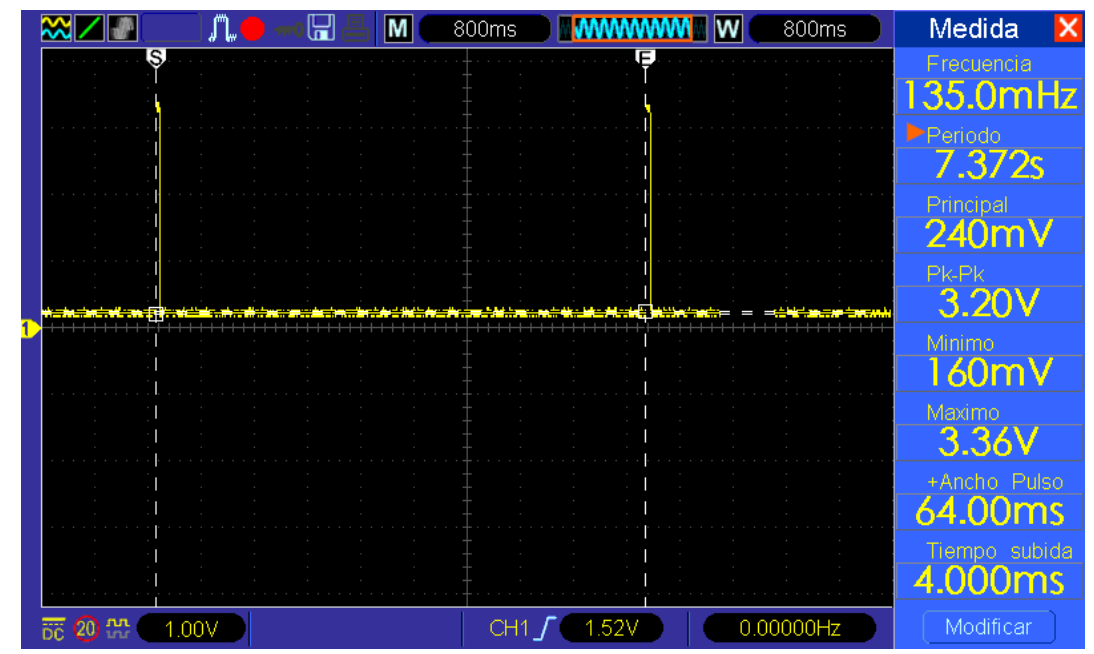

Figura 2.15. Señal de pulsos generada por la unidad Cyble Sensor.

Se ha llevado a cabo una prueba sobre contador en servicio en vivienda unifamiliar. Se ha acoplado una unidad Itron Cyble-Sensor sobre el contador modelo Flodis. Se trata de una unidad 
referencia 42K1 con salida de 2 conductores. Observando la actividad del contador se ve como en un minuto la lectura ha pasado de 739166 I a 739175 I, lo que supone un caudal de 9 I / min. Se produce una revolución de la aguja cada $7.25 \mathrm{~s}$. Los resultados obtenidos pueden verse en la Tabla 2.5 incluyendo sus correspondientes en el caso de haber utilizado un sensor con una constante mayor.

\begin{tabular}{cccccccc}
\hline & $\mathbf{m}^{3} / \mathbf{h}$ & $\mathrm{l} / \mathrm{h}$ & $\mathrm{I} / \mathrm{min}$ & $\mathrm{I} / \mathrm{sg}$ & $\begin{array}{c}\mathrm{s} / \text { pulso } \\
(\mathrm{K}=1)\end{array}$ & $\begin{array}{c}\text { s / pulso } \\
(\mathrm{K}=10)\end{array}$ & $\begin{array}{c}\text { s / pulso } \\
(\mathrm{K}=100)\end{array}$ \\
\hline $\begin{array}{c}\text { Prueba } \\
\mathbf{1}\end{array}$ & 0,12 & 120 & 2 & 0,03333333 & 30 & 300 & 3000 \\
\hline $\begin{array}{c}\text { Prueba } \\
\mathbf{2}\end{array}$ & 0,54 & 540 & 9 & 0,15 & 6,666666667 & 66,66666667 & 666,6666667 \\
\hline
\end{tabular}

Tabla 2.5. Señal de salida en función del caudal.

Se muestra a continuación una posible conexión a kits de desarrollo loT. En el caso de Waspmote de Libelium, dado que la entrada con interrupción es activa a nivel alto, hay que disponer el Cyble Sensor para que al generar un pulso, derive el nivel positivo de tensión hacía dicha entrada. Una resistencia externa a masa fija el nivel bajo en la entrada en ausencia de señal.

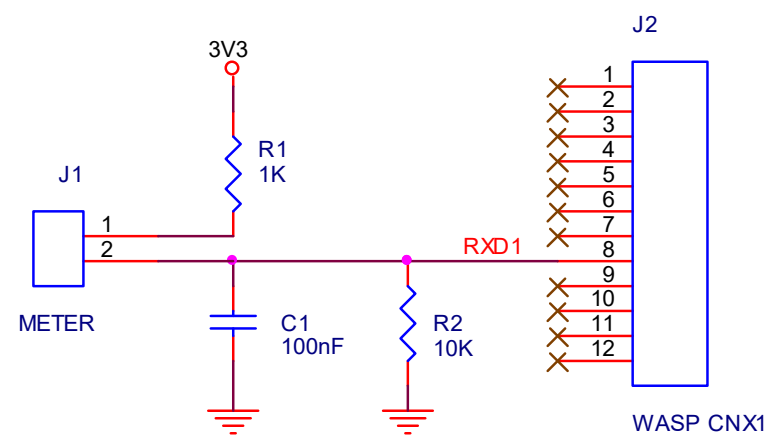

Figura 2.16. Conexión a módulo Waspmote

En el caso de conectar al módulo PyCom, este puede generar una interrupción y despertar del modo de bajo consumo detectando un nivel bajo a su entrada, por lo que la conexión del CybleSensor se realiza para cortocircuitar a masa en cada pulso. En la configuración software del módulo Pycom se activa la resistencia de pull-up en esa entrada para fijar un nivel en ausencia de salida del Cyble-Sensor.

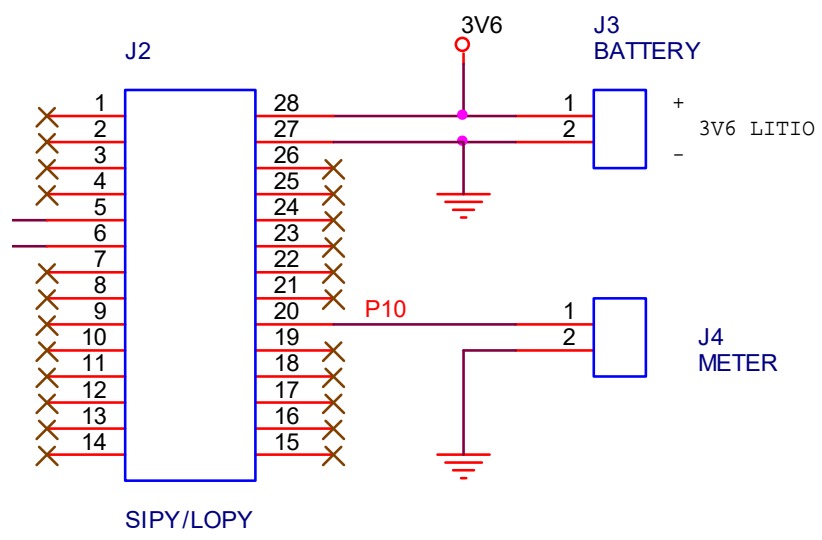

Figura 2.17. Conexión a módulo Pycom 


\subsection{Tecnologías de comunicación específicas para loT}

\subsubsection{LPWAN}

Low Power Wide Area Networks se presenta como una tecnología óptima para loT en los casos en los que se requiera gran autonomía, gran alcance, coste contenido y sea suficiente con transferir pequeñas cantidades de datos [13]. A diferencia de WiFi, Bluetooth, ZigBee o NFC, permite enlaces de mayor alcance y la escalabilidad es mayor al soportar un número de dispositivos más elevado. En comparación con tecnologías celulares como 2G, 3G o 4G, el consumo de energía es menor con lo que se aumenta la autonomía, el coste del dispositivo es más reducido y la conectividad de nuevos dispositivos es más simple [14].

Algunas soluciones como NB-IoT, estandarizado por el 3GPP (3rd generation partnership project) utiliza bandas de frecuencias bajo licencia asignadas a los operadores de telefonía móviles y aprovecha parte de su infraestructura ya desplegada [15]. A diferencia de esta, las tecnologías LPWAN que más atención están consiguiendo actualmente por parte de la industria y la comunidad investigadora son Sigfox y LoRa, que operan en bandas radio ISM (Industrial Scientific Medical). En las bandas ISM se puede operar sin licencia siempre que se cumplan las restricciones de potencia radiada, ancho de banda y ciclo de transmisión. La banda más utilizada en Europa por estas tecnologías es la de $868 \mathrm{MHz}$, definida en la ECC 70-03 [16] y que según segmento permite una ERP (Effective Radiated Power) de $14 \mathrm{dBm}$ a $27 \mathrm{dBm}$ y un ciclo de operación menor del 1\% o del 10\%. Esto implica que un mismo dispositivo no está autorizado a emitir más del 1\% cada hora en cada frecuencia, es decir 36 segundos, que en función de la duración de la transmisión del mensaje, puede suponer un máximo de 6 mensajes a la hora o lo que es lo mismo, un mensaje cada 10 minutos.

\subsubsection{Sigfox}

\subsubsection{Tecnología}

Sigfox [Sigfox, 2018b] es el nombre de una tecnología para loT y también un operador de red que proporciona una solución completa que va desde la recogida de datos procedentes de objetos en cualquier zona del mundo bajo cobertura, hasta el traspaso de dichos datos a los sistemas de información del cliente. El modelo de negocio se basa en facturar por la conectividad proporcionada a los dispositivos. Desde 2009 ha tenido un rápido crecimiento estando presente en noviembre de 2018 en 53 países con 5 millones de km² cubiertos [Sigfox, 2018c]. Cada estación base puede cubrir un área de entre 3 a $10 \mathrm{~km}$ en entornos urbanos y de 30 a $50 \mathrm{~km}$ en zonas rurales, así como dar servicio a 1 millón de dispositivos [Centenaro et al., 2016].

Para que un dispositivo pueda registrarse en la red es necesario que supere un proceso de certificación de conformidad con las especificaciones de Sigfox, sirviendo así para asegurar compatibilidad y calidad del servicio. En los test se evalúa tanto el protocolo en capa de enlace como el rendimiento de la radiación RF, dando lugar a una clasificación en función de la potencia de transmisión [Sigfox, 2018a]. Cuando el proceso es superado se concede un certificado que posteriormente será necesario para el registro de cualquier otro dispositivo del mismo modelo. Además, cada dispositivo lleva asociada una pareja de identificadores ID/PAC necesarios para el registro. El Device-ID es un identificador de 32 bits único a nivel global, está grabado en la memoria no volátil del dispositivo y no cambia durante la vida útil de este. El PAC (Porting Authorization Code) demuestra la propiedad del dispositivo y cambia cada vez que el dispositivo es registrado o transferido. 
Para transmitir la información, se utiliza en capa física tecnología radio UNB (Ultra Narrow Band) con ancho de canal de $100 \mathrm{~Hz}$, modulación DBPSK (Differential Binary Phase Shift Keying) y una tasa de bits de 100 bps [Do et al., 2014]. La modulación es la más sencilla de las de tipo por desplazamiento de fase y presenta la mayor inmunidad al ruido al ser la diferencia entre símbolos máxima, de $180^{\circ}$. Concentrando la radiación en un ancho de banda muy pequeño se aumenta la densidad de energía y se consigue un enlace más inmune a interferencias de señales con mayor ancho de banda y potencia [Lauridsen et al., 2017]. Son posibles enlaces inalámbricos de largo alcance de hasta $163.3 \mathrm{~dB}$ de perdida total con $14 \mathrm{dBm}$ de potencia de transmisión, antena con $2.15 \mathrm{dBi}$ de ganancia, sensibilidad del receptor de la estación base -142 dBm y antena de recepción de $5.15 \mathrm{dBi}$ [Sigfox, 2018d]. En la Figura 2.18 se observa que cada transmisión del dispositivo dura alrededor de $2 \mathrm{~s}$ y es repetida 2 veces más en frecuencias $6 \mathrm{kHz}$ por encima y por debajo de la inicial con objeto de aumentar las posibilidades de recepción por parte de la red [Sigfox, 2019]. Las estaciones bases mantienen recepción en un segmento de $192 \mathrm{kHz}$ en torno a la frecuencia central en Europa de $868.130 \mathrm{MHz}$ y los dispositivos pueden transmitir de forma aleatoria en cualquier frecuencia dentro de ese segmento [Sigfox, 2018e]. En caso de solicitarse respuesta, se abre una ventana de recepción de 25 s y posteriormente se efectúa una confirmación de la recepción. Para la transmisión de la red al dispositivo se utiliza modulación GFSK (Gaussian Frecuency Shift) a una velocidad de 600 bps y frecuencia 1.395 $\mathrm{MHz}$ superior a la empleada para la primera transmisión de subida, dentro siempre del segmento de bajada de $200 \mathrm{KHz}$ y frecuencia central en Europa de $869.525 \mathrm{MHz}$ [Vejlgaard et al., 2017]. Se trata por tanto de una comunicación bidireccional pero asimétrica, además de ser siempre iniciada por el dispositivo y de producirse una comunicación en sentido descendente sólo cuando este la solicita. El número de mensajes por dispositivo hacia la red está limitado a 140 al día y los de bajada a 4.

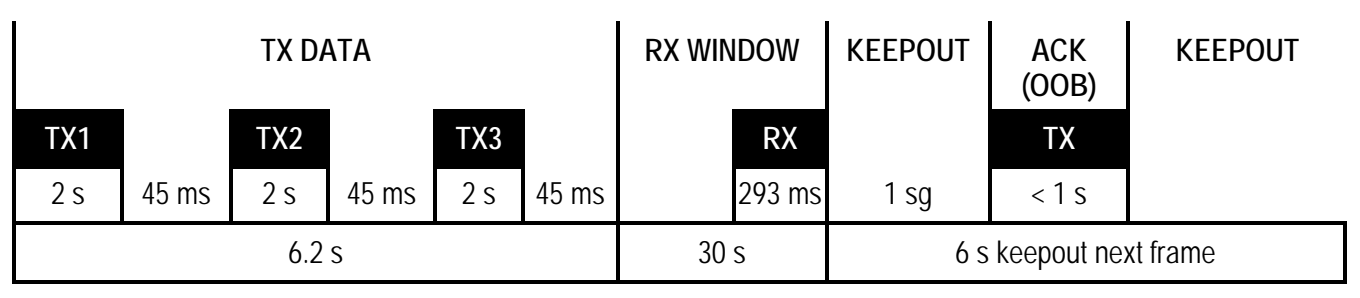

Figura 2.18. Cronología de una comunicación Sigfox.

En la capa de enlace (MAC) cada trama de subida tiene una longitud total de 26 bytes (208 bits). De estos hasta 12 bytes (96 bits) pueden ser carga útil, es decir, un mensaje definido por el usuario. La sobrecarga de protocolo consiste en un preámbulo para sincronización del receptor, el identificador de dispositivo para autenticar el mensaje, un identificador de mensaje para detectar duplicados y una suma de bits para comprobación de errores. No incluye ninguna señalización con la red ni se confirman los mensajes. El usuario es libre de distribuir como desee la información en los 12 bytes de carga útil, puesto que estos se almacenarán y llegarán a los servidores del cliente tal cual [Sigfox, 2018e]. La Figura 2.19 muestra esta trama correspondiente al nivel 2 OSI (Open System Interconnection).

\begin{tabular}{cccccc}
\hline PREAMBLE & FRAME SYNC & DEVICE-ID & PAYLOAD & AUTHENTICATION HASH & CRC \\
\hline 19 & 29 & 32 & $0-96$ & 16 & 16 \\
\hline
\end{tabular}

Figura 2.19. Trama Sigfox de subida. 
La trama de bajada en la Figura 2.20 puede contener hasta 8 bytes de información personalizables según la aplicación. El contenido puede establecerse desde el backend de Sigfox con constantes o variables tales como la fecha y hora actuales, o bien ser generado de forma dinámica desde la infraestructura del usuario.

\begin{tabular}{cccccc}
\hline PREAMBLE & FRAMESYNC & ECC & PAYLOAD & AUTHENTCATIONHASH & CRC \\
\hline 91 & 13 & 32 & $0-64$ & 16 & 8 \\
\hline
\end{tabular}

Figura 220. Trama Sigfox de bajada.

Con respecto a la seguridad, se procede a la autenticación del emisor mediante el identificador de dispositivo y a la autenticación de mensaje mediante su número de secuencia. Al no ser necesaria una negociación con la red en el momento de envío de mensajes, el dispositivo efectúa la transmisión sin esperar respuesta, con lo que intentar interferir el receptor del dispositivo no evita la comunicación. En el enlace radio no se realiza ninguna encriptación de los mensajes, esto es delegado a la capa de aplicación en caso de ser necesario. Entre las estaciones base y los servidores de Sigfox se establece un túnel VPN y en la comunicación con la infraestructura del usuario puede utilizarse el protocolo seguro HTTPS.

La topología de red Sigfox es una estrella de estrellas, conectándose múltiples objetos a una misma estación base y todas las estaciones base a los servidores de Sigfox. La estación base actúa de sumidero de datos recibidos hacia IP. La detección de duplicados y autentificación son gestionadas por el servidor de red, no por las estaciones base. Se utiliza el concepto de red colaborativa. Cada zona suele estar cubierta por 3 estaciones base en lo que se denomina diversidad espacial. Tener 3 estaciones base en 3 localizaciones diferentes cubriendo cada objeto aumenta la fiabilidad de recepción de los mensajes.

\subsection{Estaciones base Sigfox}

Una estación base es la parte de la infraestructura de red que comprende el conjunto de elementos necesarios para establecer un enlace radio con dispositivos Sigfox dentro de una determinada área de cobertura. Además realiza la función de puerta de enlace o gateway entre el segmento de red donde se encuentran los dispositivos Sigfox y una red IP que facilita el intercambio de información con los servidores de gestión y almacenamiento.

Las estaciones base de Sigfox son fabricadas y distribuidas por la misma compañía Sigfox. El despliegue es llevado a cabo por un operador de telecomunicaciones con el que se firma un acuerdo en cada país o región para propiciar la expansión global de la red.

Los elementos que componen una estación base son los siguientes:

- Antena para la banda ISM de operación de Sigfox, en el caso de Europa, 868 MHz.

- Amplificador de radiofrecuencia de bajo ruido tipo LNA (Low Noise Amplifier) para la recepción.

- Protector para descargas de rayos.

- Unidad que agrupa radio Sigfox y tarjeta de procesamiento.

- Conectividad cableada a Internet.

- Modem LTE / 3G para conectividad inalámbrica a Internet de reserva. 
Un ejemplo de antena instalada en las estaciones base Sigfox es la CXL-900-3LW del fabricante Procom. Se trata de un modelo omnidireccional de $70 \mathrm{~cm}$ de longitud y $5 \mathrm{dBi}$ de ganancia protegida con fibra de vidrio y preparada para montaje en mástil.

La unidad que contiene la radio Sigfox y la tarjeta de control y procesamiento de señal, tiene como referencia SBS-T3 para el modelo anterior y SBS T-902 para el más reciente con capacidad de montaje en rack. Ambas unidades tienen un cuerpo metálico y unos indicadores tipo LED en el frontal que permiten conocer el estado de operación y de la conexión IP.
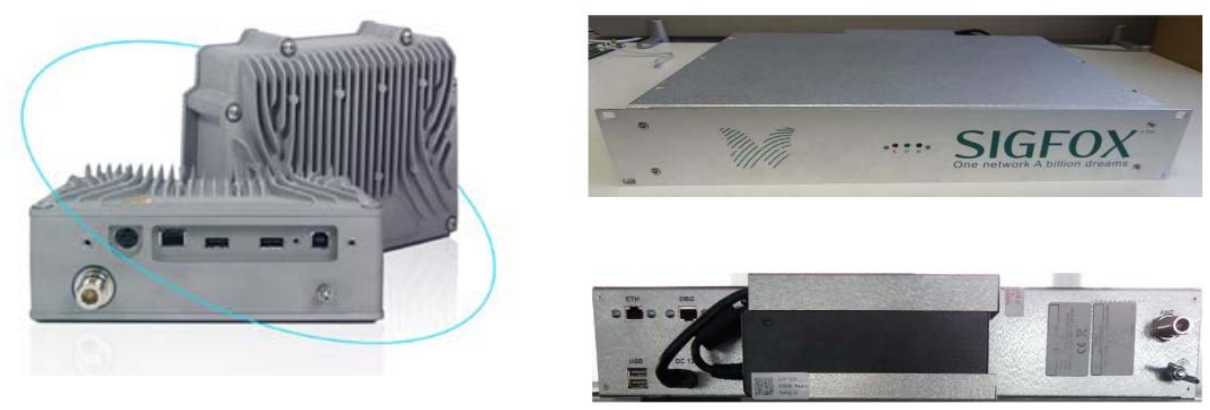

Figura 2.21. Estaciones base Sigfox SBS-T3 a la izquierda y SBS-T-902 a la derecha.

La radio incluida es capaz de recibir simultáneamente todo el ancho de banda de operación, de manera que los dispositivos finales pueden iniciar una transmisión en cualquier frecuencia elegida de forma aleatoria dentro de dicho segmento. Se trata de una radio de tipo SDR (Software Defined Radio) y es la unidad de control la encargada de detectar y decodifican las diferentes transmisiones. La señal de radiofrecuencia se hace llegar desde la antena a través de un conector de tipo $\mathrm{N}$.

La unidad de control posee conectores de alimentación por baja tensión continua, puerto de red Ethernet 100BaseT y dos puertos USB 2.0. Además el chasis posee un terminal para conectarlo a tierra. La conectividad principal se realiza mediante una conexión de red cableada al router de una línea ADSL o de fibra óptica. Como medida de backup para asegurar el servicio, en uno de los conectores USB se inserta un modem radio LTE/3G que es usado en el caso de caída de la conexión de datos primaria.

\subsubsection{Backend}

El backend de Sigfox es una plataforma accesible por todo gestor final de dispositivos Sigfox que permite el registro de nuevos dispositivos, la visualización de la información enviada por estos, la definición de tramas de bajada, así como el reenvío de mensajes a la plataforma propietaria del usuario 0 a otros servicios de gestión de información IoT disponibles en Internet. La información se encuentra estructurada en diferentes tipos de entidades cuya relación se ha representado en la Figura 2.22. 


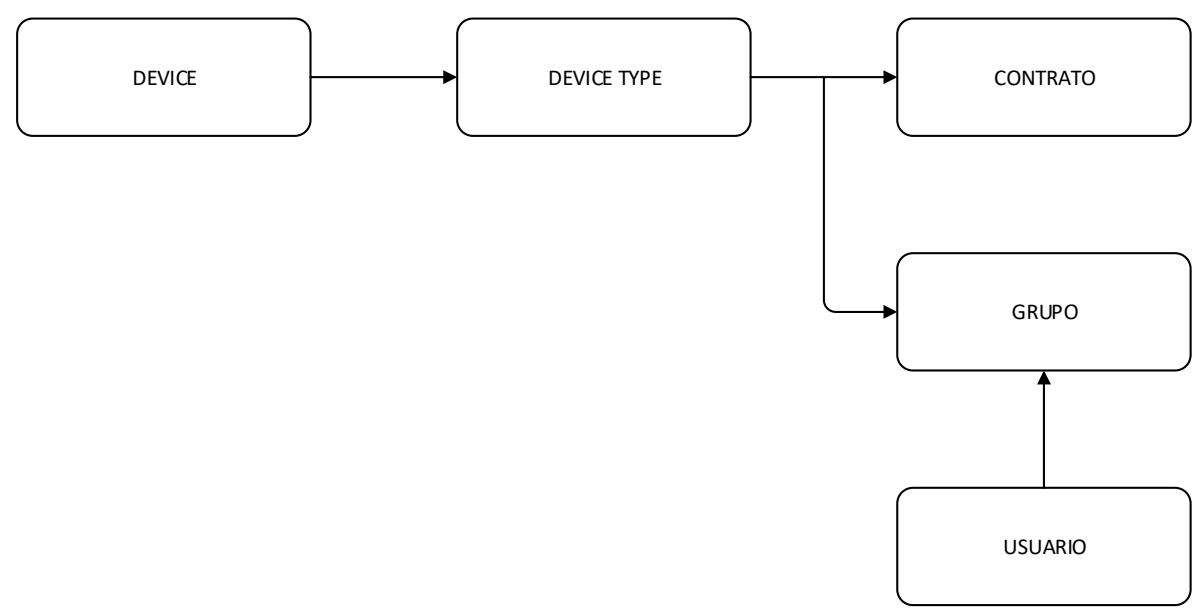

Figura 2.22. Relación entre entidades en el backend de Sigfox.

Cada una de las entidades tiene las siguientes funciónes y características:

- Device: Se refiere a un dispositivo final identificado por la terna ID / PAC / Certificado. Admite la siguiente funcionalidad:

- Visualización de mensajes y eventos.

- Visualización de la localización aproximada.

- Estadísticas de mensajes, nivel de señal RSSI y relación SNR.

- Posibilidad de lanzar eventos por llamadas URL ó eMail ante incidencias.

- Device Type: Es una agrupación de dispositivos que tienen en común:

- Contrato.

- Trama de bajada.

- Visualización personalizada de datos en la trama.

- Callback.

Mediante la edición de sus parámetros es posible personalizar lo siguiente:

- Trama de bajada y modo de envío.

- Visualización personalizada de datos.

- Keep-alive (tiempo máximo entre tramas para considerar un problema de comunicación).

- eMail de alerta.

- Estadísticas de todos los dispositivos del tipo de mensajes y SNR.

- Posibilidad de lanzar eventos por URL o eMail ante incidencias o cambios.

- Usuario. Corresponde a un nombre de cuenta con acceso a determinados dispositivos 0 información de ellos según los permisos que le hayan sido concedidos.

- Se activa tras la declaración de una dirección eMail. El usuario elige contraseña.

- Asociado a uno o varios grupos de Device types. 
- Permisos de acceso configurables.

- Grupo. Agrupación de varios Device Types y asociado a usuarios para una gestión más eficiente de grandes cantidades de objetos.

- Puede ser subgrupo de otro grupo.

- Acceso a la API.

- Posibilidad de lanzar eventos por URL/eMail ante incidencias o cambios.

El orden de creación de elementos es el siguiente:

1. Contrato.

2. Grupo.

3. Device Type. Vinculado a grupo y contrato.

4. Device. Vinculado a Device Type.

Una vez formalizado el contrato y disponibles las licencias de conexión para los nuevos dispositivos, se crea un grupo como asociación de dispositivos que van a pertenecer a un mismo proyecto y van a estar bajo la supervisión de un mismo usuario o bajo el acceso a través de una misma API. Pueden ser dispositivos con Device Types diferentes y en contratos diferentes.

Se crea al menos un Device Type por cada contrato que se quiera utilizar y uno por cada modelo de dispositivo asociado a ese contrato que pueda tener un downlink, callback o representación de datos en el backend diferente. Al crear el Device Type se solicita el grupo al que va a pertenecer y el contrato asociado.

Se declara finalmente un dispositivo nuevo y se asocia a un Device Type ya creado. Todos los dispositivos bajo un mismo Device Type tendrán el mismo tratamiento de los datos enviados y recepción de trama de bajada. Pueden transferirse más tarde dispositivos entre diferentes Device Type y con ello cambiar de contrato o de grupo. Además es posible reemplazar la configuración de un dispositivo por otro y con ello cambiar también de contrato asociado.

En la Figura 2.23 puede consultarse como el listado de dispositivos registrados es presentado en el backend, pudiéndose personalizar los campos mostrados y realizar filtrado por dichos campos. Adicionalmente puede accederse a los mensajes enviados por cada dispositivo si se hace click sobre el ID o la configuración del tipo del tipo de dispositivo si se hace click sobre el Device Type. 


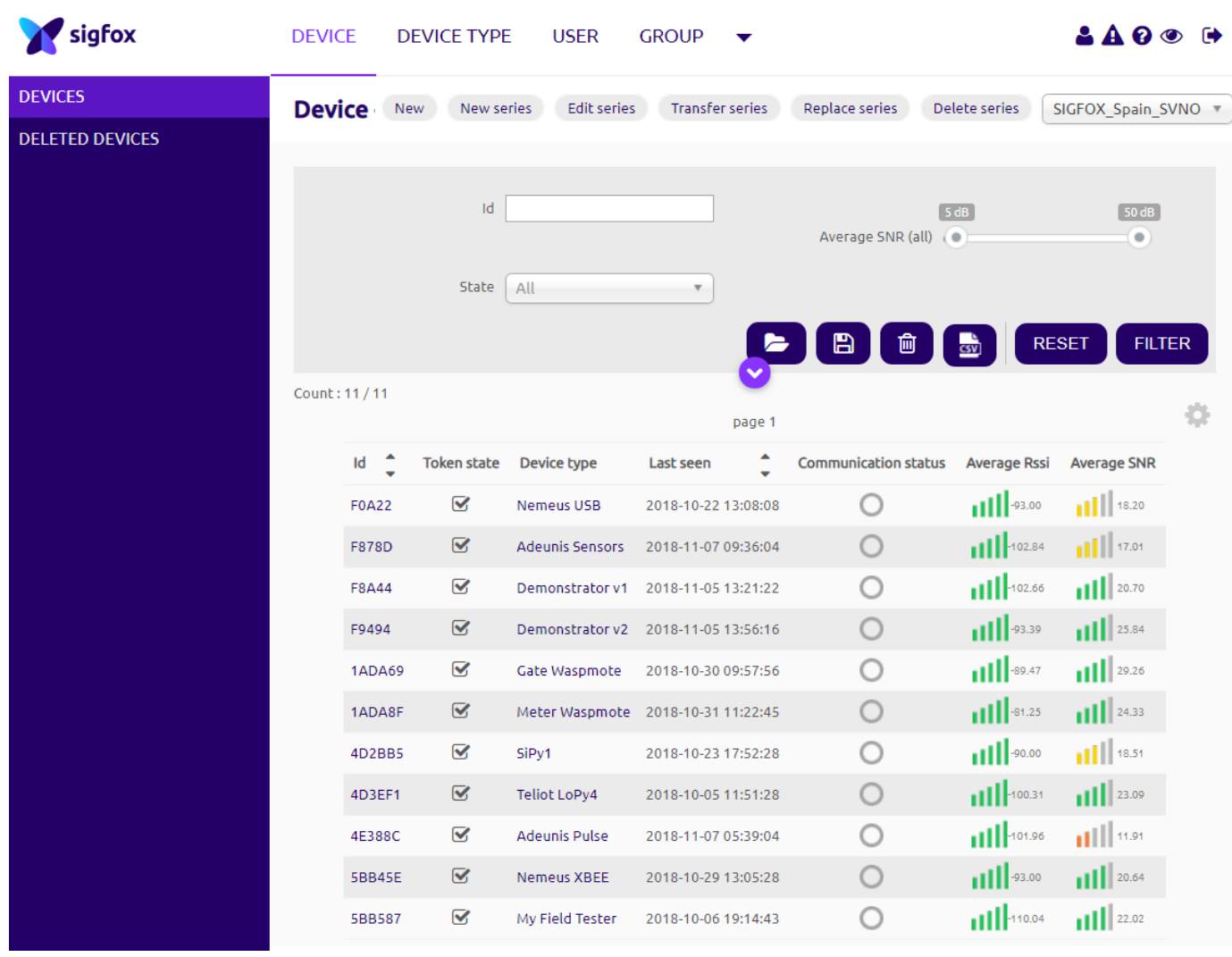

Figura 2.23. Listado de dispositivos en el backend de Sigfox.

La barra superior para selección de la pestaña activa permite conmutar entre entidades con el objeto de trabajar con cada una de ellas. Una vez elegida un tipo de entidad, las acciones que pueden realizarse sobre ella se muestran en una barra de botones superior. A través de la barra lateral se puede seleccionar el tipo de listado u otra información relacionada con la entidad. Por ejemplo, en el caso de seleccionar dispositivo, las acciones que pueden realizarse son agregar nuevos dispositivos y operaciones masivas como editar los datos de varios de ellos, transferir series entre diferentes tipos o eliminar múltiples dispositivos a la vez. A través de la barra lateral se puede seleccionar si se listan los dispositivos activos o los eliminados. Si en cambio se ha seleccionado un dispositivo concreto, las acciones serán sólo sobre este, tales como suspender la comunicación, reiniciar la secuencia de transmisión, cambiar sus parámetros o transferirlo a otro Device Type. La barra lateral también se modifica en este caso mostrando la posibilidad además de ver los mensajes enviados, la información técnica asociada al dispositivo, su localización aproximada, eventos o estadísticas.

Seleccionada la pestaña Device Type, sobre cada uno de los tipos se pueden mostrar los dispositivos asociados, estadísticas, configurar llamadas externas del tipo Callback y modificar la configuración. Esta comprende el contrato asociado, la trama de bajada junto al modo de hacerla llegar, el valor máximo del temporizador de presencia, así como el formato de los datos enviados por los dispositivos de este tipo para su correcta visualización en el listado del backend.

Otras entidades como los usuarios tienen menos posibilidades, únicamente crear nuevos asociándolos a un grupo y asociándole permisos, o cambiar la información de los ya existentes tal como el email y la contraseña. En el caso de los grupos, pueden crearse nuevos seleccionando otro al que pertenecerán, o editarse el nombre y la descripción de los ya 
existentes. Las opciones en la barra lateral permiten visualizar los usuarios, tipos de dispositivo y contratos asociados a ese grupo, así como la configuración de eventos y acceso a la API.

El registro y activación de nuevos dispositivos difiere de si se trata de kits de desarrollo con licencia incluida o productos comerciales que no suelen llevarla y requieren adquirir licencias adicionales mediante un contrato con Sigfox. El procedimiento en el caso de kits de desarrollo consiste en acceder a la URL: https://backend.sigfox.com/activate, seleccionar la marca y el modelo de dispositivo, para finalmente introducir el ID, PAC y certificado del mismo. Esta información la proporciona el fabricante. En el caso necesitar vincular el dispositivo a un contrato previo a su registro en el backend, una vez formalizado el contrato, se ha de crear un Device Type asociándolo a este contrato y por último crear un nuevo dispositivo registrándolo con su ID, PAC y certificado. En el momento del registro se asociará al Device Type previamente creado y a su vez asociado al contrato correspondiente.

En los listados de mensajes recibidos se muestra un indicador gráfico de señal recibida o RSSI por cada mensaje. Consiste en 5 barras con diferentes colores. Los rangos son los siguientes: Color rojo si la señal está entre $-142 \mathrm{dBm}$ y $-135 \mathrm{dBm}$, clasificado como un nivel límite. Color amarillo si la señal está entre $-135 \mathrm{dBm}$ y $-122 \mathrm{dBm}$, clasificado como niveles medios y color verde para valores superiores a $-122 \mathrm{dBm}$, clasificados como niveles buenos 0 excelentes. En el caso de un listado por tipos de dispositivo, el valor mostrado corresponde a un valor medio del nivel de señal con el que se han recibido los últimos 25 mensajes.

La trama de bajada enviada a un dispositivo cuando este transmite y solicita una confirmación, puede definirse en el backend y quedará de forma estática hasta que se modifique nuevamente, o también puede variarse a través de la API. Se define una trama de bajada diferente por cada tipo de dispositivo, esto permite cambiar la configuración de múltiples de ellos de hardware similar o participando en un mismo proyecto. El modo de bajada puede seleccionarse entre directo, con lo que se enviará una trama de radiofrecuencia al dispositivo cuando este la solicite, o realizar una llamada de tipo callback a un servidor externo del cliente. La carga útil de bajada puede tener un máximo de 8 bytes y se definen en formato hexadecimal. Los valores dependen de cada aplicación concreta, aunque también existen algunas variables predefinidas como \{time\} que ocupa 4 bytes y transmite la fecha y hora actual en formato Unix Epoch. El identificador de la estación base, también 4 bytes, puede enviarse utilizando \{tapid\}, así como el nivel de señal recibida con \{rssi\} en 2 bytes. Por último, \{roaming\} de 1 byte es un valor booleano que indica si el dispositivo está en su red de origen o utilizando otra de las disponibles a nivel global.

Los datos recibidos de cada dispositivo se listan en la página de mensajes tal y como se reciben, sin embargo, puede hacerse una interpretación simple del contenido a efectos de visualización, indicando el tipo de cada uno de los datos que componen la trama y su longitud. Esto puede hacerse por cada tipo de dispositivo editando su configuración y en el apartado Payload display indicando que se va a hacer una personalización con la opción Custom grammar. De manera general se define un nombre de variable, su posición en la trama, el tipo de dato, su longitud y opcionalmente si el orden de los bits es inverso. Los tipos de datos que pueden representarse son booleanos, enteros con y sin signo, de coma flotante y caracteres ASCII representables. La documentación referente a todos los posibles tipos de datos admitidos y sus opciones, se encuentra en la ayuda de la propia plataforma backend junto al cuadro para introducir la cadena de configuración. En la Figura 2.24 se incluye un ejemplo de representación personalizada de los datos enviados por uno de los dispositivos diseñados en este trabajo. 


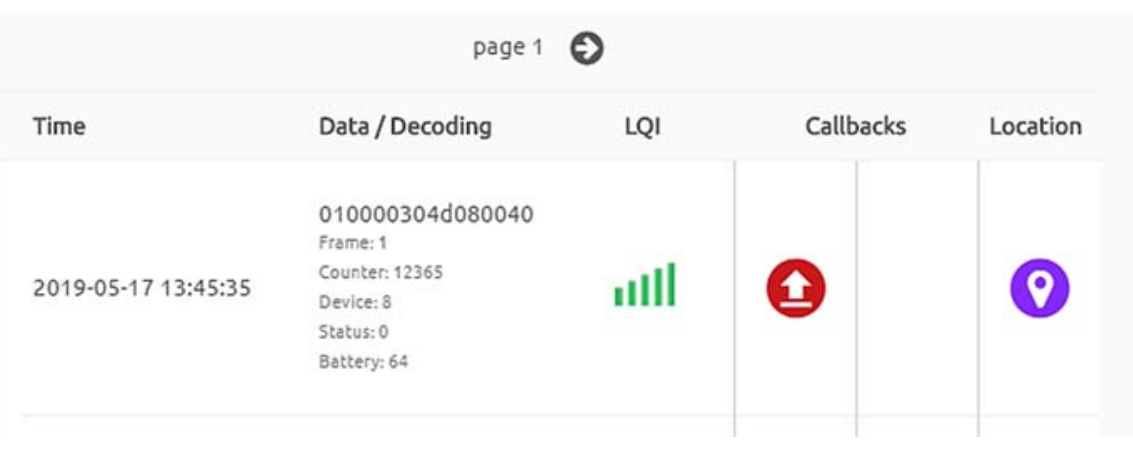

Figura 2.24. Representación personalizada en el backend de datos recibidos.

Para ese resultado, la correspondiente gramática personalizada consiste en un byte de tipo entero sin signo indicando el tipo de trama, un valor de contador de 32 bits 04 bytes sin signo, así como el identificador de dispositivo, el código de error y el nivel de batería todos ellos de un byte de longitud. La sintaxis de la configuración es la siguiente:

Frame::uint:8 Counter::uint:32 Device::uint:8 Status::uint:8 Battery: : uint : 8

Un ejemplo de trama más compleja correspondiente al dispositivo comercial Adeunis Pulse ARF8048PA, se muestra a continuación. En este caso un mismo byte contiene información diversa como el número de secuencia de trama y el código de error, además de que el valor de contador está invertido siendo enviado primero el bit de menor peso, por lo que se ha de especificar que la notación es little-endian.

Frame-Type: :uint:8 Frame-Index:1:uint:4::7 Error:1:uint:4::3 PulseType: :uint:8 Counter: :uint:32:little-endian

Los datos recibidos de cada dispositivo pueden ser enviados por el backend a la infraestructura propia del usuario o a una plataforma intermediaria específica para loT donde sean almacenados y/o tratados. Para llevar a cabo esta tarea se utiliza una transferencia denominada callback que consiste en hacer una llamada al servidor de destino tras la recepción de cada mensaje de un dispositivo. Esta configuración se define por cada tipo de dispositivo. Un tipo común de callback es una llamada tipo HTTP con métodos GET o POST seleccionables y formato configurable según variables predefinidas como \{time\}, \{device\} o \{data\} y otras definibles por el usuario en la configuración de visualización personalizada antes descrita. Un ejemplo de patrón URL para la llamada callback es el que se utiliza en los dispositivos puestos en activo en este trabajo mostrada a continuación:

http://212.128.45.110:2780/sigfox-

in . php?device $=\{$ device $\} \&$ time $=\{$ time $\} \&$ station $=\{$ station $\} \& s n r=\{$ snr $\} \& r s s i=\{r$ ssi $\} \&$ data $=\{$ data $\}$

Las opciones disponibles para reorganización de dispositivos en el backend permiten la transferencia de estos entre contratos, por ejemplo en el caso de kits de desarrollo que han estado con una licencia de evaluación y ahora quieren pasarse a un contrato propio. También es posible el traspaso de dispositivos entre grupos o de un tipo de dispositivo a otro mediante la opción Transfer. Estas operaciones no siempre se llevan a cabo de inmediato y pueden pasar a una cola de tareas pendientes. 
Por último, con la API REST incluida, puede visualizarse la información de los dispositivos y modificarse la configuración desde una infraestructura externa sin tener que acceder con una cuenta de usuario. Para ello se ha de habilitar en Group > API Access > New tras lo cual se generará un usuario y contraseña. Entre otras posibilidades, puede modificarse la configuración del Device Type, incluyendo la trama de bajada o las diferentes llamadas Callback.

\subsubsection{LORaWAN}

\subsubsection{Tecnología}

LoRa (Long Range modulation technique) [Semtech, 2018a] es una solución para redes LPWAN basada en técnicas de transmisión de espectro ensanchado y modulación CSS (Chirp Spread Spetrum). Fue ideada en 2010 por la startup francesa Cycleo, la cual fue adquirida por el fabricante de semiconductores Semtech Corporation en 2012, quien actualmente es el propietario de la tecnología, tiene registrada la marca LoRa y mantiene todos los derechos. El modelo de negocio consiste en obtener beneficios por el chipset radio LoRa, dejando al usuario 0 a un intermediario el despliegue y gestión de la red que da cobertura a los objetos conectados. La especificación LoRa sólo describe la capa física (PHY). Como capa de enlace (MAC) está disponible LoRaWAN (LoRa for Wide Area Networks), la propuesta de código abierto de la Alianza LoRa que puede ser utilizada libremente en desarrollos propios tanto de dispositivos como de gateways. La Figura 2.25 muestra la organización en capas de LoRa y LoRaWAN. El protocolo LoRaWAN incluye prestaciones de encriptación, autenticación, velocidad adaptativa y corrección de errores [Sanchez-lborra et al., 2018].

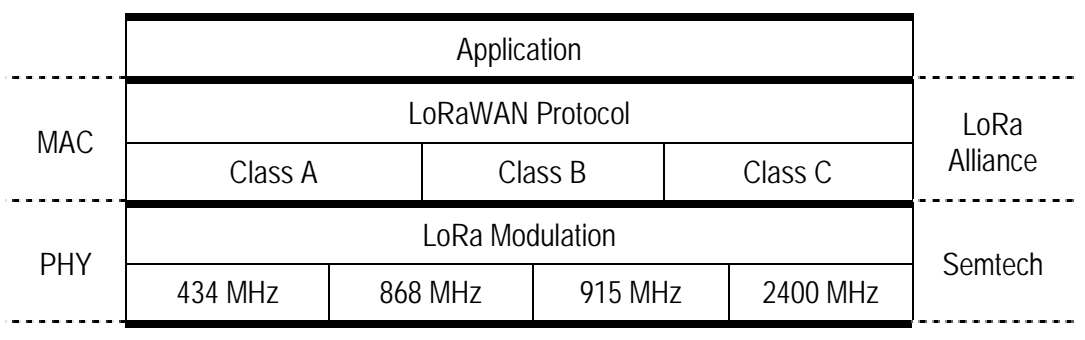

Figura 2.25. Modelo de capas para LoRa y LoRaWAN.

El chip radio cubre las frecuencias entre 150 y $960 \mathrm{MHz}$ permitiendo operar en la mayoría de bandas ISM de todo el mundo. La modulación LoRa utiliza una señal llamada chirp que cambia de frecuencia continuamente barriendo todo el ancho del canal radio que tipicamente es de 125 $\mathrm{KHz}$. Ajustando los parámetros Spreading Factor (SF), Bandwidth (BW) y Coding Rate (CR) puede llegarse a una solución de compromiso entre la velocidad de datos, el link budget, la inmunidad a interferencias y la ocupación del espectro. Cada bit de información está representado por múltiples barridos o simbolos, y la relación entre la velocidad de transmisión de símbolos y la duración del barrido es el Spreading Factor, con valores típicos de 7 a 12 correspondiendo a velocidades nominales de 11000 a 250 bps también denominadas Data Rate (DR0-DR6). A valores más altos de SF se reduce la velocidad efectiva de transferencia pero se incrementa la robustez del enlace. También con objeto de incrementar esta, se utiliza codificación de error cíclica con posibles valores desde 4/5 a 4/8 para detección y corrección de errores suponiendo un ratio de sobrecarga de entre 1.25 y 2 . El uso de un mayor ancho de banda permite aumentar la velocidad, pero también reduce la sensibilidad y está condicionado por la normativa de cada región [Semtech, 2018b]. 
La tecnología LoRa es capaz de demodular señales $19.5 \mathrm{dBm}$ por debajo del nivel de ruido, a diferencia de la mayoría de sistemas utilizando modulación FSK (Frecuency Shift Keying) que necesitan una señal 8-10 dBm por encima del nivel de ruido, pudiéndose conseguir distancias de $6 \mathrm{~km}$ en entornos urbanos y de $18 \mathrm{~km}$ en escenarios rurales con pérdida total de enlace de hasta $154 \mathrm{dBm}$ y sensibilidad del receptor de $-142 \mathrm{dBm}$ [Feltrin et al., 2018]. Una habilidad añadida de LoRa es la capacidad de demodular varias señales simultáneamente en la misma frecuencia si tienen diferente SF, creándose así diferentes canales virtuales e incrementando la capacidad del gateway. En Europa se establecen 3 canales de obligada operación para todos los gateways en $868.100,868.300$ y $868.500 \mathrm{MHz}$. Además pueden definirse otros según política de operación de cada red.

Se definen 3 categorías de dispositivos [LoRa Alliance, 2018]:

- Clase A: Se mantienen en ahorro de energía la mayor parte del tiempo. Tras el envío de información mantienen una ventana de recepción y vuelven a reposo. No pueden recibir datos en cualquier momento.

- Clase B: Comunicación a intervalos regulares sincronizada con una baliza. Aunque no necesiten enviar datos, despiertan periódicamente para recibir de la red.

- Clase C: Enlace continúo con la red. Pueden recibir y enviar datos en cualquier momento.

En la Figura 2.26 se muestran los campos que componen una trama de subida LoRaWAN tanto a nivel físico como a nivel de capa MAC. Puede verse en la Figura 2.27 que la trama de bajada que no incluye comprobación de integridad a nivel físico para mantener los mensajes tan cortos como sea posible y reducir su impacto en las limitaciones de ciclo de operación en las bandas ISM. La carga útil puede ser como máximo de entre 51 y 222 bytes dependiendo del SF y tanto en el enlace ascendente como descendente [Adelantado et al., 2017]. Cada dispositivo posee un identificador único y permanente de 32 bits denominado DevEui. LoRaWAN incluye seguridad y autenticación basada en el esquema de encriptación AES128 (Advanced Encryption Standard) y otros estándares de seguridad descritos en el IEEE 802.15.4/2006. A diferencia de otros sistemas que dependen de una sola clave para ambas funciones, LoRaWAN separa entre autenticación y encriptación. La clave AppSKey se utiliza para encriptar la carga útil de los mensajes. La autenticación y control de integridad del mensaje usa la clave de sesión de red NwkSKey. Existen dos métodos de asociación del dispositivo a la red [Robert Miller, 2018]:

- ABP (Activation By Personalization): Con este método se necesita programar en el dispositivo las claves de seguridad. Basado en el DevEUI, el servidor genera para cada cliente un DevAddr, NwkSKey y AppSKey que habrán de utilizarse durante la comunicación.

- OTAA (Over The Air Activation): En este caso el dispositivo ya contiene la información necesaria para unirse a la red y sólo es necesario registrar sus DevEui y AppKey en el servidor. En cada solicitud de asociación el dispositivo envía los identificadores DevEui y AppEui. El servidor genera las claves AppSKey, NwkSKey y las envía al cliente junto al DevAddr para ser utilizadas durante el resto de la comunicación. 


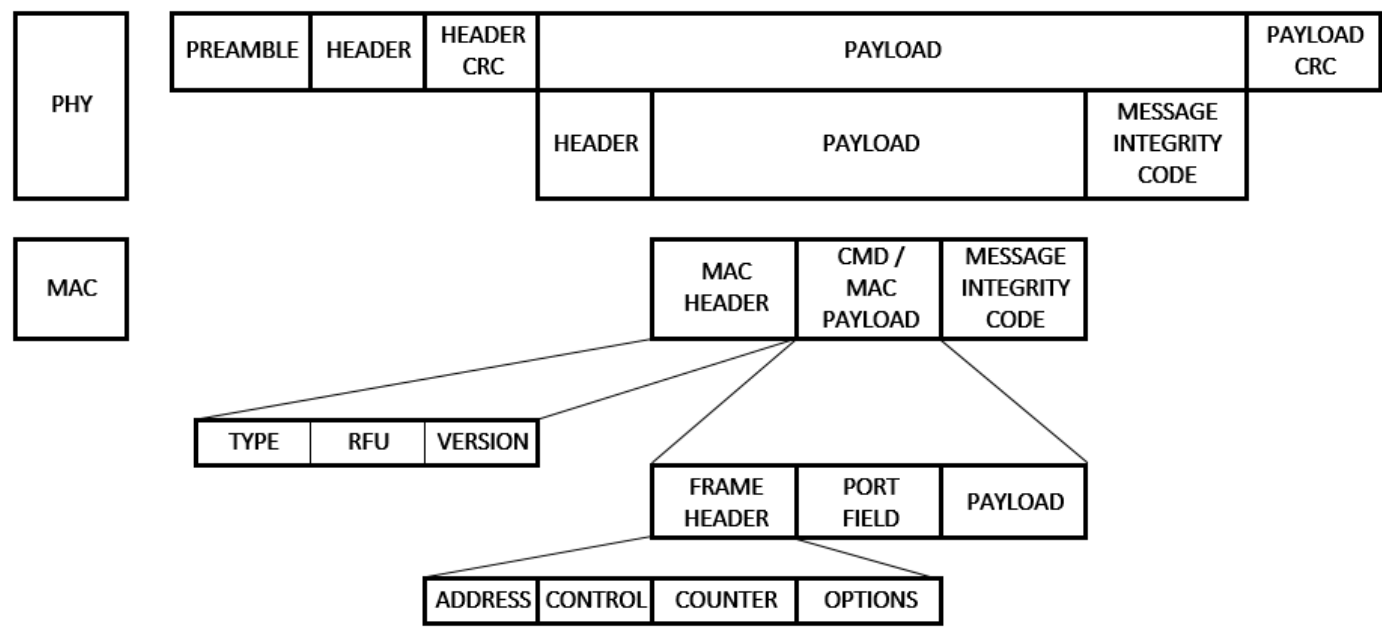

Figura 2.26. Trama LoRaWAN de subida. Campos que la componen.

\begin{tabular}{|l|l|l|l|l|l|c|}
\hline PREAMBLE & HEADER & $\begin{array}{c}\text { HEADER } \\
\text { CRC }\end{array}$ & \multicolumn{4}{|c|}{ PAYLOAD } \\
\hline & & & HEADER & PAYLOAD & $\begin{array}{c}\text { MESSAGE } \\
\text { INTEGRIY } \\
\text { CODE }\end{array}$ \\
\hline
\end{tabular}

Figura 2.27. Trama LoRaWAN de bajada. Campos que la componen.

La topología de red es de una estrella de estrellas. Múltiples dispositivos pueden acceder a través de un mismo gateway y todos los gateway están enlazados vía IP al servidor de red. El gateway hace las funciones de bridge agregando información extra como el nivel de la señal recibida, pero no se requiere asociación alguna con él. Varios gateway pueden recibir y reenviar la información de un mismo objeto y es el servidor de red el que se encarga de filtrar duplicados, autenticar los dispositivos y seleccionar el gateway más apropiado para el enlace de bajada [Margelis et al., 2015].

\subsubsection{Gateways comerciales LoRaWAN}

Se encuentran disponibles en el mercado diversos productos comerciales capaces de establecer un enlace radio con dispositivos IoT LoRaWAN, recoger los datos aportados por estos y reenviarlos a un servidor a través de otra red del tipo IP. Por su función de intercambio de información entre redes de distinta naturaleza reciben la denominación de gateway.

Se diferencian entre sí por el entorno de operación para el que han sido diseñados, existiendo tanto de interior como de instalación en exteriores. Los de uso en interiores son de carcasa plástica y aspecto similar a un router WiFi, con antena interna y conectividad WiFi o Ethernet. Aquellos diseñados para exterior se suministran en una carcasa metálica y grado de protección IP67 para superar las inclemencias meteorológicas, conexión de descarga de rayos y anclajes para mástil. La conectividad suele venir dada por una conexión cableada de tipo Ethernet y/o un modem integrado de tipo 4G ó 3G con antenas externas. La antena principal para la banda de 
$868 \mathrm{MHz}$ suele ser omnidireccional con ganancia de $3 \mathrm{dBi}$ o $6 \mathrm{dBi}$, construida en fibra de vidrio y su señal de RF se hace llegar a través de un conector del tipo $\mathrm{N}$.

Otra característica diferenciadora es la posible funcionalidad adicional, ya que además de la función de gateway algunos son capaces de ejecutar software con aplicaciones personalizadas por el usuario para el enrutado de la información o el tratamiento de los datos recibidos. Algunos son incluso capaces de ejecutar un servicio LoRaWAN para el registro y gestión de los dispositivos.

Algunos ejemplos de productos disponibles en el mercado y que se han considerado para el despliegue en el entorno real, son:

Del tipo montaje en interior:

- Cisco : 910 Industrial Router.

- Link Labs : LL-BST-8 LoRa Gateway.

- CALAO Systems : Toti-LoRa-pico.

Del tipo montaje en exterior:

- MultiTech : MultiConnect Conduit IP67 Base Station.

- Kerlink : LoRa IoT Station.

- Cisco : Interface Module for LoRaWAN + Router IR809 / IR829 (3 sect).

- Rishight : RHF2S008.

- Kona : Macro loT Gateway.

Para su utilización en el ámbito de este trabajo y posibles investigaciones futuras, se valoran positivamente las siguientes características:

- Montaje en exterior.

- Antena + accesorios montaje incluidos.

- Robustez / Acabado industrial.

- Facilidad de configuración.

- Operación como packet-forwarder.

- Lora Network Server.

- Posibilidad de $433 \mathrm{MHz}$.

- Coste contenido.

En base a ello, los candidatos seleccionados han sido la Kerlink Wirnet Station 868, la Multitech Conduit IP67 y la Cisco IR910. Finalmente, la decisión de compra ha sido a favor del modelo Multitech Conduit por las siguientes razones:

- Carcasa para exterior con acabado robusto.

- Incluye accesorios montaje y antena.

- Buen soporte en España y completa documentación oficial.

- Distribuidor en España.

- Node-Red App incluida.

- LoRa Network Server incluido (AEP y Mlinux).

Están disponibles dos versiones de esta estación base, una con mLinux e interface de usuario en línea de comandos, y otra denominada AEP con interface Web. Se ha seleccionado la segunda 
para una más rápida puesta en marcha y en la Figura 2.28 puede verse una captura de pantalla de la interface Web presentada al usuario a efectos de parametrización.

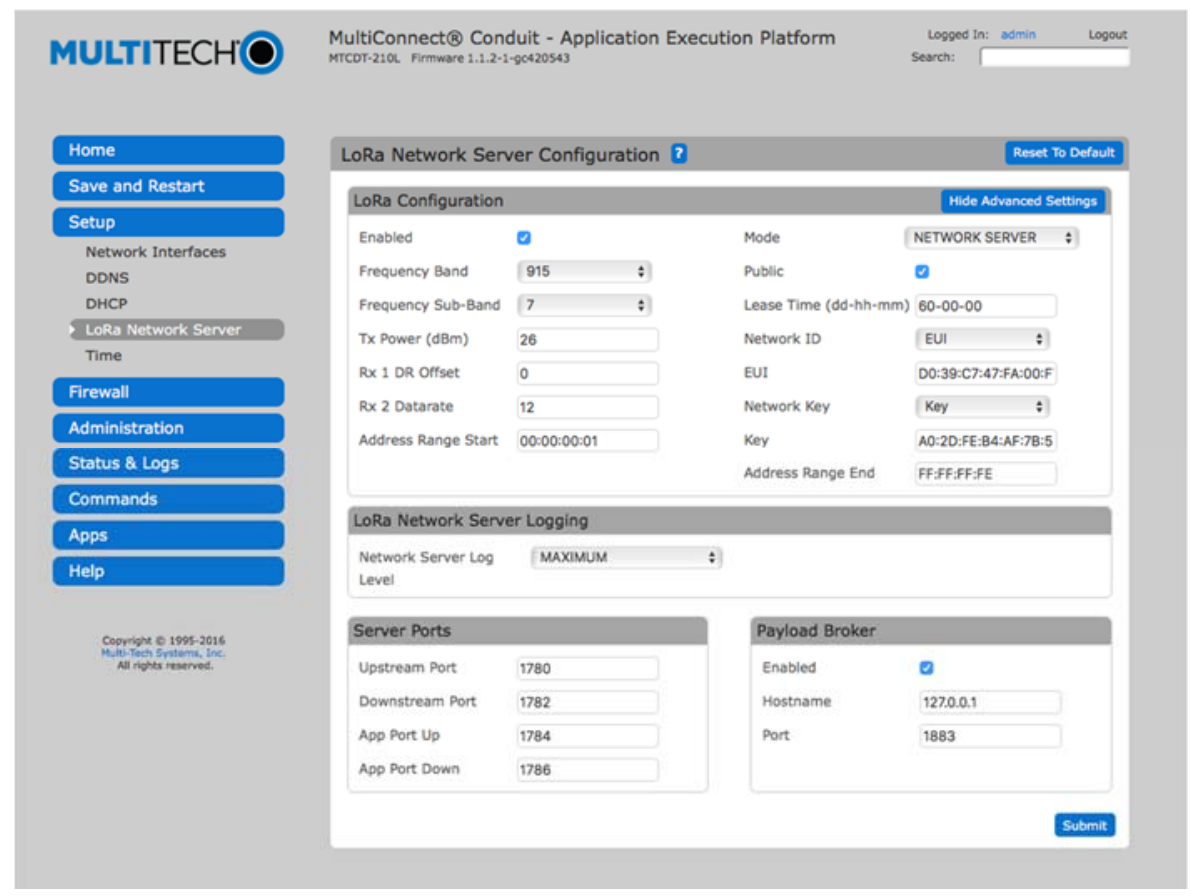

Figura 2.28. Interface de usuario de la estación base Multitech Conduit.

Puede verse también en la Figura 2.29 la estación base una vez instalada en la azotea del edificio ELDI de la Universidad Politécnica de Cartagena para llevar a cabo las pruebas de cobertura y el caso de estudio propuesto en este trabajo.

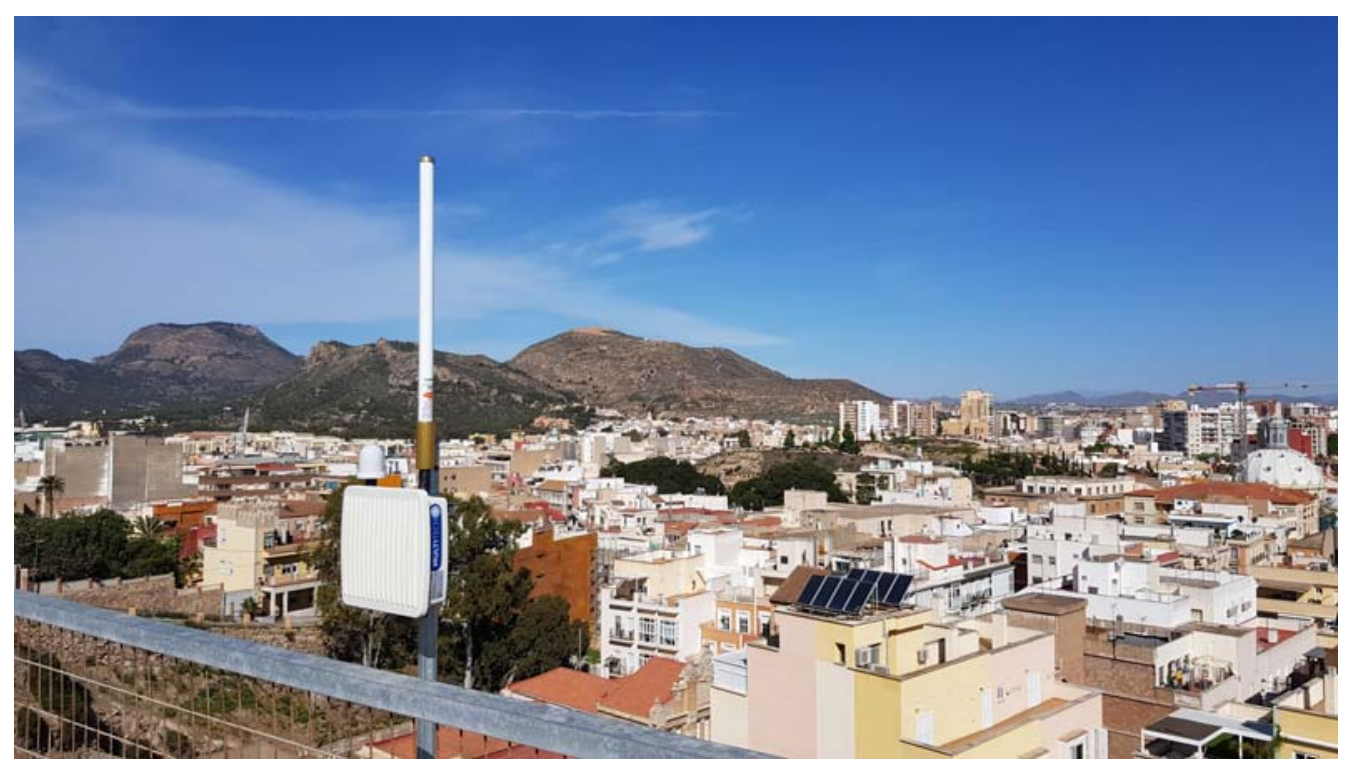

Figura 2.29. Estación base Multitech Conduit una vez instalada. 


\subsubsection{Plataforma LORIOT}

El servicio LORIOT proporciona una plataforma software para crear y operar una infraestructura del tipo LoRaWAN sin necesidad de desplegar servidores propios para la gestión de los dispositivos loT.

Permite registrar nuevos dispositivos capaces de asociarse a la red tanto por los métodos de autenticación ABP como OTAA y registrar los gateways que enlazan con los dispositivos y pasan sus datos a LORIOT. En el caso de los dispositivos, habrá que registrarlos en LORIOT a partir del identificador de dispositivo y la clave de aplicación proporcionadas por el fabricante si van a trabajar con autenticación OTAA. En el caso de ABP, la plataforma puede generar las claves de red, de sesión y de aplicación que se habrán de configurar en el dispositivo. Para el registro de gateways es necesario introducir en LORIOT la dirección MAC de estos y en los gateways configurar como destino la IP del servidor LORIOT correspondiente a la zona. El servicio ofrecido por LORIOT es capaz también de gestionar el control adaptativo de velocidad y potencia de dispositivos compatibles LoRaWAN. La información recibida y decodificada puede ser transferida de múltiples formas a la infraestructura del usuario o a una plataforma de terceros para el tratamiento y almacenamiento de la información. Una de las formas de transferir los datos es generar llamadas de tipo callback mediante HTTP al servidor configurado por el usuario. El modelo de negocio es el cobro del servicio en función del número de dispositivos finales como de gateways registrados. Es posible evaluar el servicio creando una cuenta libre que actualmente permite el registro de un gateway y 10 dispositivos finales.

En la Figura 2.30 puede verse una captura de pantalla de la interface Web ofrecida por LORIOT y en la que se aprecia un listado de dispositivos registrados con su identificador, nombre, nivel de señal y relación señal-ruido con que han sido recibidos por el gateway, factor de ensanchamiento utilizado, nivel de batería, estado de activación del control de potencia adaptativo, la clasificación del tipo de dispositivo, número de secuencia de tramas recibidas y tiempo transcurrido desde la última vez que fue recibido.

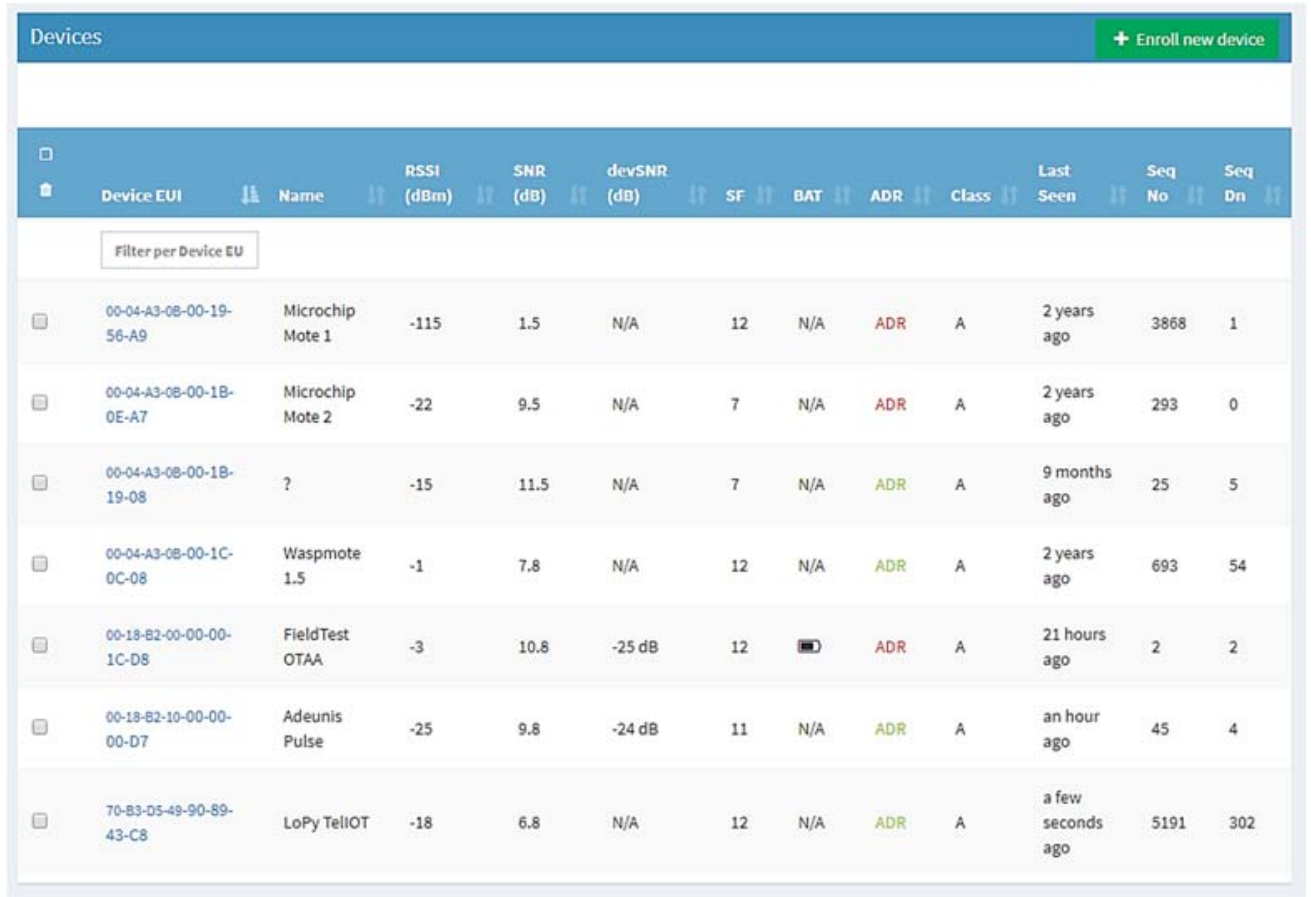

Figura 2.30. Interface de usuario de LORIOT. 
Para comprobar el correcto funcionamiento de la plataforma y su interacción tanto con dispositivos loT como con otros servidores externos, se ha configurado una aplicación de prueba de manera que llevase a cabo una llamada HTTP a un servidor propio al recibir cada trama de datos. En la máquina destino se ha activado un servicio HTTP con Apache, una base de datos mySQL y se ha programado con PHP una pequeña utilidad capaz de extraer la información recibida por el método GET de HTTP, formatearla adecuadamente e insertarla en la correspondiente tabla de una base de datos. El resultado fue satisfactorio.

Podemos concluir que la funcionalidad de LORIOT es, en el entorno LoRaWAN, el equivalente al backend proporcionado por Sigfox, dejando ya únicamente al usuario la responsabilidad en el tratamiento y almacenamiento de la información enviada por los dispositivos.

\subsection{Kits de desarrollo para comunicaciones loT}

Se encuentran disponibles en el mercado diferentes kits y módulos útiles para evaluar las comunicaciones IoT de tipo LPWAN como Sigfox y LoRaWAN, además de poder desarrollar en un breve periodo de tiempo nuevos casos de aplicación.

\subsubsection{Kit de evaluación LoRaWAN de Microchip}

El kit de evaluación de Microchip para LoRaWAN referencia DV164140-1, incluye como puede verse en la Figura 2.31, dos dispositivos finales denominados motes y una unidad mayor que hace la función de gateway. Los motes incluyen un procesador de tipo PIC con una aplicación de ejemplo que envía datos periódicamente, pero también puede utilizarse para programar una aplicación de usuario a través del puerto USB. En su pantalla LCD puede visualizarse el estado de operación y con dos pulsadores es posible configurar algunos parámetros. Incluye un sensor de temperatura para enviar datos reales. La antena incluida es de tipo externo mediante un conector de tipo RSMA. La alimentación puede ser a través del puerto USB o mediante baterías de tipo AA en el porta-pilas ubicado en la parte inferior.

Por su parte, la placa que lleva a cabo la función de estación base o gateway, se divide en dos interconectadas, una de ellas con la parte de control y comunicaciones, y la otra con la parte radio. En la placa de control hay una pantalla de tipo TFT a color donde se puede visualizar el estado de operación, incluyendo tramas recibidas y enviadas así como el porcentaje de éxito. La alimentación proviene de un conector USB y la conectividad IP se realiza por un Ethernet de tipo RJ45. En la placa de radio se encuentra un chip concentrador de Semtech capaz de gestionar varias frecuencias simultáneamente y dos de chips de radio también de Semtech. La conexión de antena es mediante RSMA y varios LEDs indican si se está recibiendo o transmitiendo. 


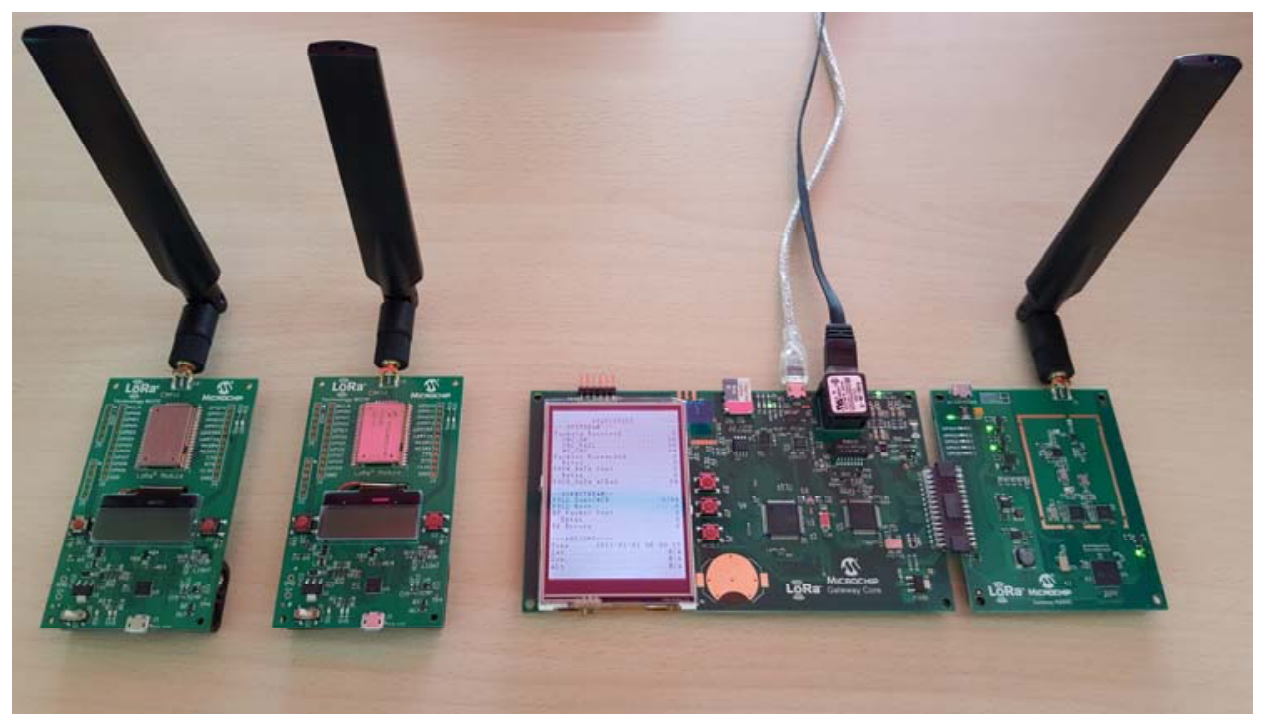

Figura 2.31. Kit de desarrollo LoRaWAN de Microchip.

Durante la fase de evaluación de la tecnología LoRaWAN, este kit de desarrollo ha sido asociado satisfactoriamente al servidor LORAWAN registrándolo primero como gateway y luego configurando las claves de los motes. También se ha utilizado como una estación base interna a nivel de laboratorio asociándolo al servidor propio para probar los dispositivos loT desarrollados en este trabajo.

\subsubsection{PyCom LoPy4}

El módulo LoPy4 de Pycom ofrece por un coste muy contenido soporte para aplicaciones IoT con un procesador muy capaz, que integra multiples opciones de conectividad y que presenta la facilidad de programación mediante un intérprete de MicroPython. Tienen formato DIL (Dual In Line Package) para un rápido prototipado e incluyen un procesador Expressif, radio ISM de 868 $\mathrm{MHz}$ compatible Sigfox y LoRaWAN con conector externo U.FL externo, radio WiFi y Bluetooth en $2.4 \mathrm{GHz}$ con antena intergrada y conector externo U.FL., memoria RAM y no volátil. La alimentación es de entre $3.4 \mathrm{Vdc}$ y $5.5 \mathrm{Vdc}$. Incluye además un puerto USB para programación y alimentación y un LED de tipo RGB para depuración. El firmware integrado incluye un servidor FTP para cargar las aplicaciones de usuario.

La programación del firmware en el dispositivo se lleva a cabo con el editor Atom de código abierto, apoyado por un complemento o plug-in para comunicar con los módulos Pycom llamado Pymakr.

Su gran ventaja es lo compacto que resulta un diseño con él, ya que al incluir procesador, memoria y conectividad, son necesarios pocos componentes adicionales según la aplicación del usuario. Existen además varias placas de ampliación oficiales que proporcionan características adicionales como sensores o un receptor GPS. Aunque incluye capacidades Sigfox y LoRaWAN, ambos modos no pueden utilizarse simultáneamente ya que comparten la radio en $868 \mathrm{MHz}$. Mediante software se ha de elegir la operación en un modo u otro. Es posible también una transmisión más convencional utilizando modulación FSK.

Algunas de sus características más destacables son las siguientes: 
Energía:

- Rango de voltaje de alimentación: 3.3V - 5.5V.

- Salida regulada de $3.3 \mathrm{~V}$ y hasta $400 \mathrm{~mA}$.

- Consumo de radio WiFi: 12 mA en modo activo, 5uA en reposo.

- Consumo de radio Sigfox/LoRa: 12 mA en modo activo, 5 uA en reposo.

Procesador:

- $\quad$ Chipset Espressif ESP32.

- Sistema on-chip de doble procesador y radio WiFi.

- El procesador de red maneja la conectividad WiFi y la pila IP v6.

- El procesador principal queda totalmente libre para ejecutar el código de usuario.

- Un coprocesador extra puede monitorizar las entradas y salidas digitales, canales ADC y controlar la mayoría de los periféricos internos durante el modo de sueño profundo con solo 25 uA de consumo.

- Memoria RAM: $512 \mathrm{kB}$.

- Memoria Flash: 4 MB.

- GPIO: Hasta 24.

- Aceleración de coma flotante por hardware.

- Python multi-hilo.

- RTC a $32 \mathrm{kHz}$.

Interfaces:

- Entradas y salidas digitales: Hasta 24.

- Puertos serie UART: 2.

- Buses digitales: 2xSPI, I2C, I2S.

- Interface para tarjeta micro-SD.

- Convertidores analógico-digital: 8XADC 12 bit.

- Temporizadores: 4 de 16 bits con PWM y captura de la entrada.

- Acceso directo a memoria (DMA) en todos los periféricos.

Comunicaciones:

- Wi-Fi : Antena interna / externa con U.FL

- Bluetooth LE : Antena interna / externa con U.FL

- LPWAN: Sigfox / LoRaWAN : Antena externa con U.FL

En la Figura 2.32 puede verse el módulo Pycom de manera independiente y en la placa de ampliación disponible del mismo fabricante con conectores adicionales para las señales de entrada y salida. La carcasa plástica también es la disponible de manera oficial. 

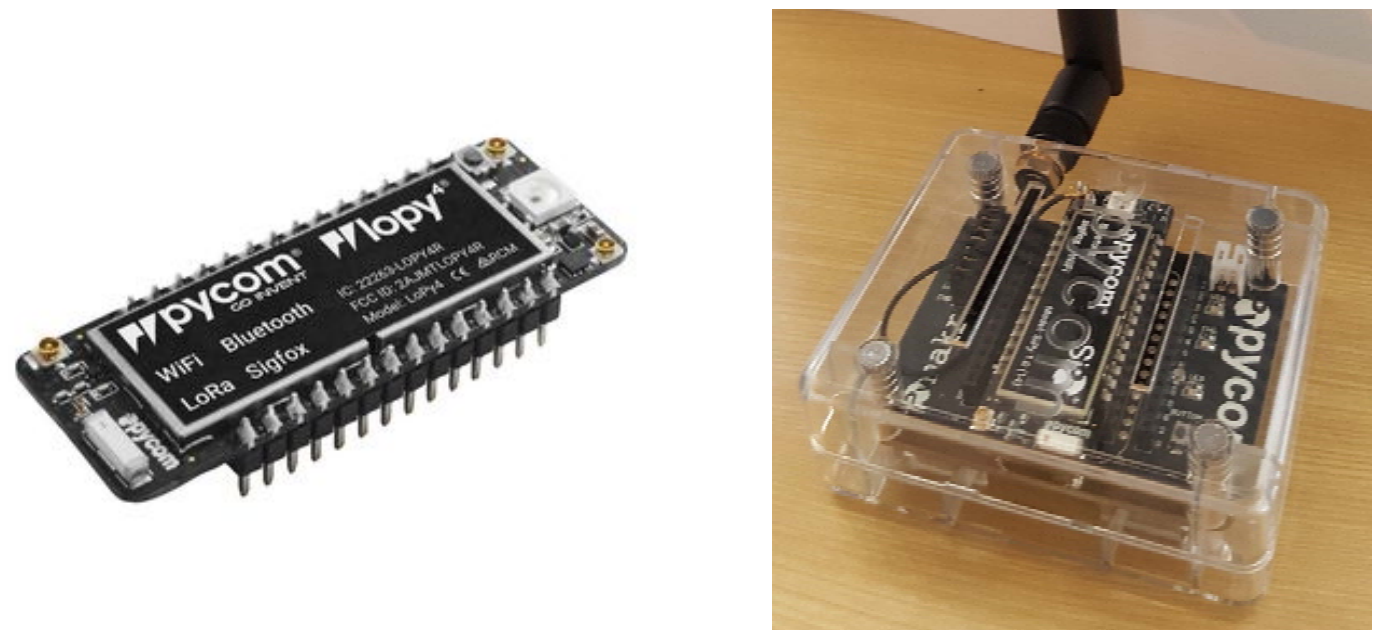

Figura 2.32. Módulo LoPy4 de Pycom y placa de desarrollo.

\subsubsection{Arduino MKRFOX1200 y MKRWAN1300}

Estas unidades acercan la comunicación Sigfox (MKRFOX1200) y LoRaWAN (MKRWAN1300) al entorno hardware y software de Arduino con las ventajas de sencillez y rápido prototipado que ello conlleva. El factor de forma es el de los módulos Arduino MKR, más reducido que el de Arduino UNO y, a diferencia de este, la tensión de alimentación y de I/O es de $3.3 \mathrm{Vdc}$ en lugar de $5 \mathrm{Vdc}$. La antena incluida en el kit es de tipo externa y adhesiva, pudiendo ser sustituida por otra gracias al conector U.FL de la placa.

El entorno de desarrollo es el mismo IDE (Integrated Development Environment) del resto de placas de Arduino, por lo que si ya se ha utilizado, la curva de aprendizaje tiene una pendiente mínima, sólo condicionada al empleo de las funciones específicas de Sigfox.

Sus especificaciones técnicas más destacas son las siguientes mostradas en la Tabla 2.6.

\begin{tabular}{ll}
\hline Característica & Valor \\
\hline Microcontrolador & SAMD21 Cortex-M0+ 32bit ARM MCU \\
\hline Alimentación & $5 \mathrm{~V}$ \\
\hline Baterías soportadas & $2 \times \mathrm{AA}$ or AAA \\
\hline Voltaje de operación & $3.3 \mathrm{~V}$ \\
\hline Pines digitales I/O & 8 \\
\hline PWM & $12(0,1,2,3,4,5,6,7,8,10, \mathrm{~A} 3$ o 18, A4 0 19) \\
\hline UART & 1 \\
\hline SPI & 1 \\
\hline I2C & 1 \\
\hline Entradas analógicas & $7(\mathrm{ADC} 8 / 10 / 12 \mathrm{bit})$ \\
\hline Salidas analógicas & $1(\mathrm{DAC} 10 \mathrm{bit})$ \\
\hline Interrupciones Externas & $8(0,1,4,5,6,7,8, \mathrm{~A} 1 \mathrm{0} \mathrm{16,} \mathrm{A2} \mathrm{0} \mathrm{17)}$ \\
\hline Corriente por pin I/O & $7 \mathrm{~mA}$ \\
\hline Memoria Flash & $256 \mathrm{kB}$ \\
\hline SRAM & $32 \mathrm{kB}$ \\
\hline EEPROM & $\mathrm{NO}$
\end{tabular}




\begin{tabular}{ll} 
Velocidad de reloj & $32.768 \mathrm{kHz}(\mathrm{RTC}), 48 \mathrm{MHz}$ \\
\hline Potencia en antena & De $13 \mathrm{a} 20 \mathrm{dBm}$ \\
\hline Frecuencia central & $868 \mathrm{MHz}$ \\
\hline Región & EU \\
\hline Longitud & $67.64 \mathrm{~mm}$ \\
\hline Ancho & $25 \mathrm{~mm}$ \\
\hline Peso & $32 \mathrm{~g}$ \\
\hline
\end{tabular}

Tabla 2.6. Especificaciones técnicas del Arduino MKRFOX1200.

En la Figura 2.33 puede verse una imagen del módulo MKRFOX1200 con la antena para 868 $\mathrm{MHz}$ y pinchado sobre una placa de prototipado. En ella también se ha utilizado un sensor de temperatura y humedad DHT22. Con este montaje se ha llevado a cabo una verificación de las prestaciones del módulo y un envío periódico a través de Sigfox de los datos proporcionados por el sensor.

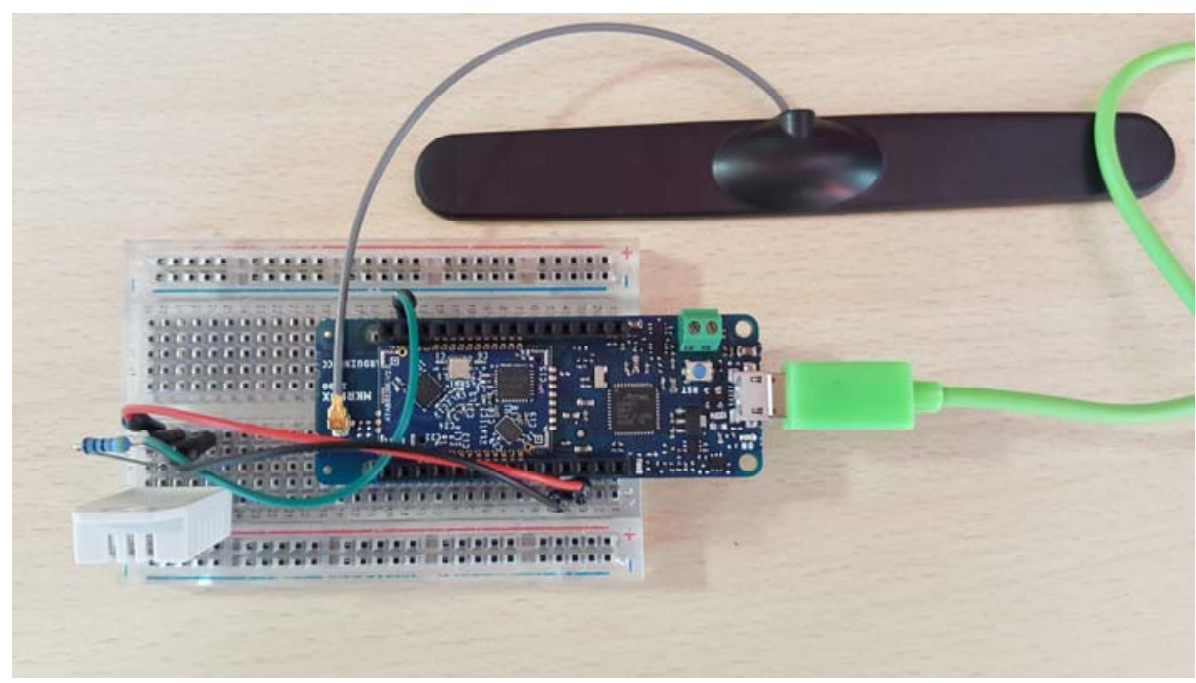

Figura 2.33. Arduino MKRFOX1200.

Utilizando el módulo MKRFOX1200 junto a la placa de expansión MKR Relay Shield también de Arduino y con referencia TSX00003, se ha puesto a prueba la capacidad de este módulo para la aplicación de telelectura y telecontrol perseguida en este trabajo. La placa MKR Relay Shield permite una más cómoda conexión de elementos externos al incluir regletas para cableado con fijación a tornillo. Además incluye un relé que puede ser utilizado para las tareas de telecontrol. Se ha utilizado el emulador de contador de consumo de agua construido en este trabajo y descrito en el capítulo 3 como generador de la señal de entrada aplicada al pin A1. En la Figura 2.34 puede verse el conjunto durante la fase de pruebas. 


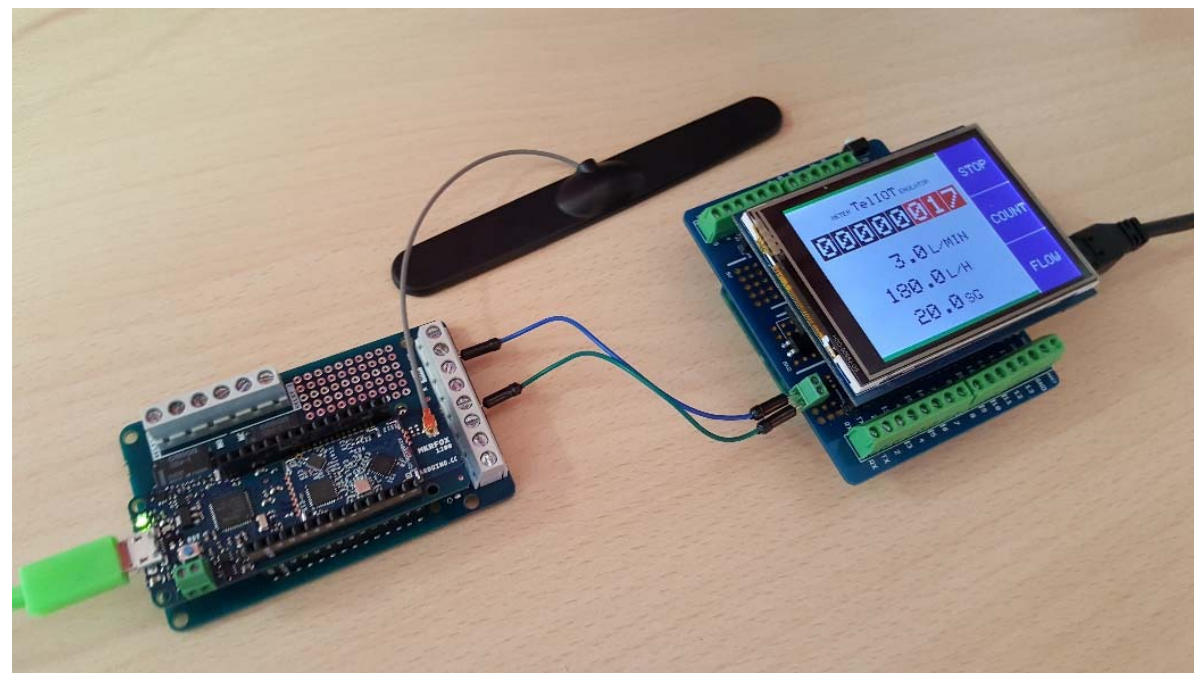

Figura 2.34. Arduino MKRFOX1200 tratando pulsos de contador.

Durante las pruebas se pudo verificar que el módulo era capaz de contabilizar correctamente la señal de entrada y de enviarla a través de la red Sigfox como puede comprobarse en la captura de pantalla del backend de sigfox con los datos recibidos en la Figura 2.35. Sin embargo, el mayor inconveniente es que el relé integrado, de pequeñas dimensiones, sólo es capaz de manejar potencias muy reducidas, por lo que sería necesario igualmente circuitería adicional externa para controlar una electroválvula.

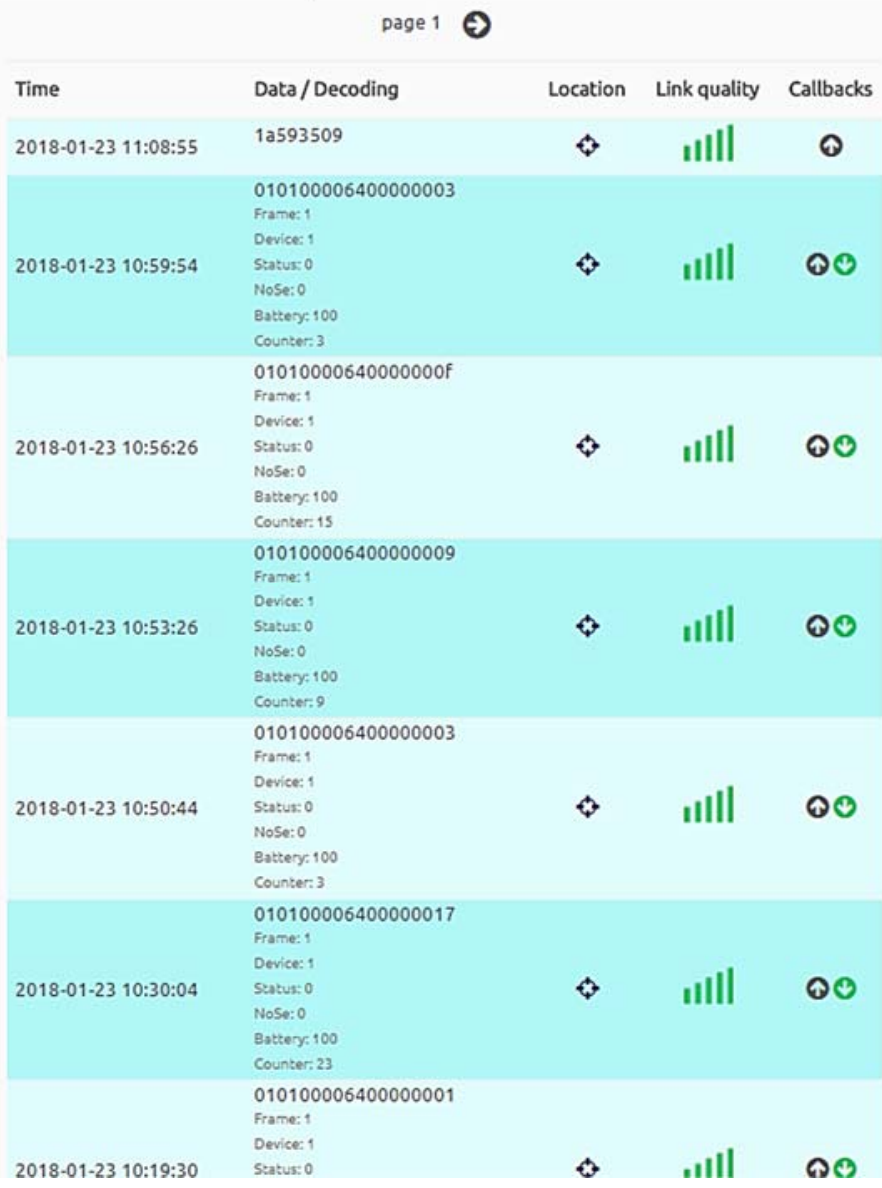

Figura 2.35. Datos recibidos en el backend de Sigfox del MKRFOX1200. 


\subsubsection{Waspmote}

La placa Wasmote de Libelium [Libelium, 2018] fue ideada pensando en el desarrollo de aplicaciones IOT. Por ello dispone de varios modos de ahorro de energía que le permiten una elevada autonomía estando alimentada mediante batería. Además, tiene disponibles una gran variedad de módulos opcionales de comunicaciones, entre los que se incluyen Sigfox y LoRaWAN, así como placas para permitir la conexión inmediata de un gran número de sensores. Un conector de expansión en la propia placa permite el que las placas adicionales puedan apilarse y quedar solidarias a la principal. En el apartado software se emplea para su programación un entorno de desarrollo basado en el popular IDE de Arduino, en el que además se incluyen librerías para todas las posibles placas de expansión. Esto hace que el tiempo de puesta en marcha del nuevo desarrollo loT sea muy reducido. Se trata de una solución de coste superior al de otras alternativas y soportada casi en exclusiva por el fabricante.

En la Figura 2.36 se encuentra una vista de la placa Waspmote con el módulo de comunicaciones Sigfox pinchado en el slot de tipo XBee. En la zona izquierda de la placa puede apreciarse el conector de expansión para placas de sensores 0 actuación. En este conector y mediante un adaptador a XBee, puede conectarse un segundo módulo de comunicaciones, siendo la única solución de las analizadas capaz de mantener dos enlaces simultáneos empleando Sigfox y LoRaWAN.

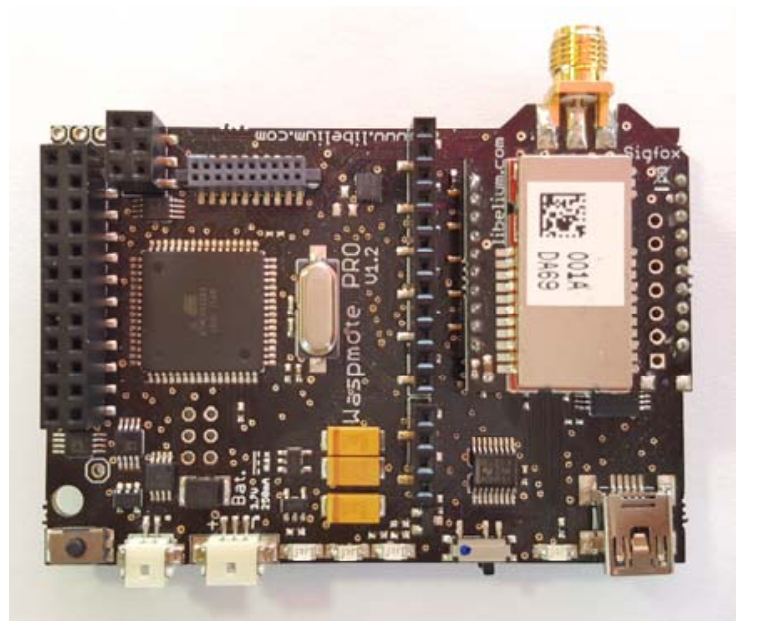

Figura 2.36. Placa de desarrollo Waspmote con módulo Sigfox.

Otra característica destacable de esta placa y útil en el ámbito del loT con objeto de dar una mayor autonomía a los desarrollos, es la inclusión de un cargador de baterías de Litio y de una entrada para panel solar. De este modo, la batería puede alimentar a la placa pero también ser recargada tanto desde el conector USB como desde un panel solar.

Sus especificaciones técnicas más relevantes se muestran en la Tabla 2.7.

\begin{tabular}{ll}
\hline Característica & Valor \\
\hline Microcontrolador & ATmega1281 \\
\hline Frecuencia & $14.74 \mathrm{MHz}$ \\
\hline SRAM & $8 \mathrm{kB}$ \\
\hline EEPROM & $4 \mathrm{kB}$ \\
\hline FLASH & $128 \mathrm{kB}$ \\
\hline
\end{tabular}




\begin{tabular}{ll}
\hline Tarjeta SD máxima: & $16 \mathrm{~GB}$ \\
\hline Peso & $20 \mathrm{~g}$ \\
\hline Dimensiones & $73.5 \times 51 \times 13 \mathrm{~mm}$ \\
\hline Rango de temperatura & $-30{ }^{\circ} \mathrm{C} \mathrm{a}+70{ }^{\circ} \mathrm{C}$ \\
\hline Reloj & $\mathrm{RTC} 32 \mathrm{kHz}$ \\
\hline Consumo ON & $17 \mathrm{~mA}$ \\
\hline Consumo en Sleep & $30 \mu \mathrm{A}$ \\
\hline Consumo en Deep Sleep & $33 \mu \mathrm{A}$ \\
\hline Consumo en Hibernación & $7 \mu \mathrm{A}$ \\
\hline Entradas analógicas & 7 \\
\hline Entradas/salidas digitales & 8 \\
\hline Comunicaciones & $\mathrm{UART}, \mathrm{I2C}, \mathrm{SPI}, \mathrm{USB}$ \\
\hline Sensores & $\mathrm{Acelerómetro}$ \\
\hline Tensión de batería & $3.3 \mathrm{~V} \mathrm{a} 4.2 \mathrm{~V}$ \\
\hline Carga con panel solar & $6-12 \mathrm{~V} / 300 \mathrm{~mA}$ \\
\hline
\end{tabular}

Tabla 2.7. Especificaciones técnicas de Waspmote.

\subsection{Comprobador de campo}

Los dispositivos del fabricante Adeunis aquí descritos, tanto en versión Sigfox (ARF8121AA) como en versión LoRaWAN (ARF8123A), permiten comprobar sobre el terreno la posibilidad de establecer comunicación IoT y verificar el área de cobertura de una red en particular. Se trata de dispositivos de mano con antena externa, tal y como puede verse en la Figura 2.37.

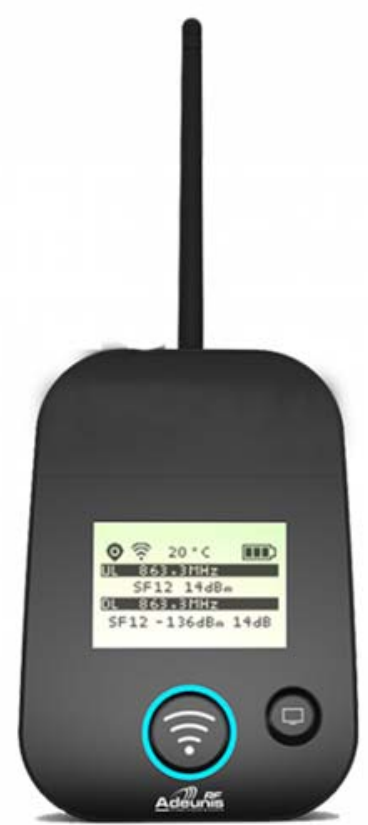

Figura 2.37. Comprobador de campo Adeunis. 
Es posible transmitir de manera automática periódicamente o en cualquier momento mediante la actuación sobre un pulsador. La potencia de salida es de $14 \mathrm{dBm}$ y la sensibilidad de recepción de $-126 \mathrm{dBm}$ en Sigfox y $-140 \mathrm{dBm}$ en LoRaWAN. La pantalla LCD retro-iluminada muestra el estado de operación y el nivel de señal y relación señal-ruido con que se ha recibido a la red. Incluye un receptor GPS cuya información de posicionamiento puede ser enviada en la transmisión IoT y así poder geolocalizar cada uno de los puntos donde se ha llevado a cabo una prueba. La alimentación proviene de una batería interna de Litio recargable por el puerto USB. Este puerto también puede ser utilizado para configurar el dispositivo. Este quizás es el mayor inconveniente, ya que muchos parámetros de configuración sólo pueden alterarse modificando los correspondientes registros desde una conexión con un PC a través del puerto USB.

Para poder configurar el dispositivo, los parámetros de la comunicación serie virtual a través de USB son 115200 bps, 8N1 y sin control de flujo. Por defecto el dispositivo envía por el puerto serie cada trama recibida. Si se quiere configurar ha de pasarse a modo de comando, modificar el correspondiente registro y grabar los cambios. Las posibilidades son:

- Activar o desactivar el receptor GPS.

- Modificar el intervalo de tiempo entre transmisiones.

- Activar o desactivar la solicitud de ACK.

- Datos a enviar.

- Frecuencia central de TX

- Frecuencia central de RX

- Numero de reintentos si se espera ACK

Puede personalizarse la información a enviar en cada transmisión, aunque por defecto se envía una trama como la representada en la Figura 2.38 conteniendo temperatura, posición, contadores de tramas y nivel de batería.

\begin{tabular}{|c|c|c|c|c|c|c|c|c|c|c|c|c|c|}
\hline Order & 1 & 2 & \multicolumn{4}{|c|}{3} & \multicolumn{3}{|c|}{4} & 5 & 6 & 7 & 8 \\
\hline Number of bytes & 1 & 1 & \multicolumn{4}{|c|}{4} & \multicolumn{3}{|c|}{4} & 1 & 1 & 1 & 2 \\
\hline Description & Status & Temperature & \multicolumn{4}{|c|}{ GPS Latitude } & \multicolumn{3}{|c|}{ GPS Longitude } & $\begin{array}{c}\text { GPS } \\
\text { Quality }\end{array}$ & $\begin{array}{c}\text { UL Coun- } \\
\text { ter }\end{array}$ & $\begin{array}{c}\text { DL Coun- } \\
\text { ter }\end{array}$ & $\begin{array}{c}\text { Battery } \\
\text { level }\end{array}$ \\
\hline Example & $9 \mathrm{C}$ & $1 \mathrm{~B}$ & 45 & 15 & 96 & \begin{tabular}{|l}
90 \\
\end{tabular} & 00 & 55 & \begin{tabular}{|l|l|}
34 & 50 \\
\end{tabular} & 27 & 20 & 20 & $\mathrm{OF}$ \\
\hline
\end{tabular}

Figura 2.38. Trama enviada por el comprobador de campo Adeunis.

Los datos de aplicación contenidos en la trama de bajada pueden mostrarse en la pantalla LCD, sin embargo, sólo se visualizan correctamente si se trata de caracteres ASCII imprimibles.

Para completar la información proporcionada por el dispositivo y no estar limitados a lo mostrado en la pantalla del mismo, se ha configurado el backend de Sigfox para que reenvíe sus tramas a un servidor propio. En él, se ha montado un servicio Web con Apache para recibir las llamadas del backend y se ha programado una utilidad en PHP que extrae los datos de posición GPS de la trama y junto al nivel de señal RSSI, los almacena en una base de datos mySQL. Otra utilidad programada también en PHP, permite leer esta base de datos y crear un archivo en formato KML con los puntos donde se ha transmitido con el demostrador y sus niveles de señal asociados tanto en forma numérica como con un color de icono diferente. 


\subsection{Conclusiones}

Previo a cualquier proceso de telelectura de la información de consumo de un contador de agua residencial, ha de considerarse si este dispone de salida de datos para tal fin, bien en forma de impulsos o de cualquier otro tipo. En caso de no disponer de ella, será necesario acoplar al contador un dispositivo intermedio que detecte su actividad y genere esta señal para su posterior tratamiento y transmisión mediante módulos de comunicaciones específicos según la tecnología deseada. Algún modelo de contador también integra conectividad, pero en este caso habrá que tener en cuenta el tipo de tecnología usada y si se trata de una solución propietaria del mismo fabricante o puede integrarse en redes loT ya existentes.

Low Power Wide Area Networks se presenta como una tecnología óptima para loT en los casos en los que se requiera gran autonomía, gran alcance, coste contenido y sea suficiente con transferir pequeñas cantidades de datos. A diferencia de WiFi, Bluetooth, ZigBee o NFC, permite enlaces de mayor alcance y la escalabilidad es mayor al soportar un número de dispositivos más elevado. En comparación con tecnologías celulares como 2G, 3G o 4G, el consumo de energía es menor con lo que se aumenta la autonomía, el coste del dispositivo es más reducido y la conectividad de nuevos dispositivos es más simple. A diferencia de esta, las tecnologías LPWAN que más atención están consiguiendo actualmente por parte de la industria y la comunidad investigadora son Sigfox y LoRaWAN, que operan en bandas radio ISM que se pueden operar sin licencia siempre que se cumplan las restricciones de potencia radiada, ancho de banda y ciclo de transmisión. La banda más utilizada en Europa por estas tecnologías es la de $868 \mathrm{MHz}$.

Sigfox es el nombre de una tecnología para loT pero también un operador de red que proporciona una solución completa que va desde la recogida de datos procedentes de objetos en cualquier zona del mundo bajo cobertura, hasta el traspaso de dichos datos a los sistemas de información del cliente. LoRa es una tecnología loT de Semtech Corporation basada en técnicas de transmisión de espectro ensanchado y modulación CSS. A diferencia de Sigfox, el modelo de negocio consiste en obtener beneficios por el chipset radio, dejando al usuario 0 a un intermediario el despliegue y gestión de la red que proporciona cobertura radio. Para ello es necesario la adquisición de estaciones base con función de gateway como la Multitech Conduit IP67 con capacidades también de ejecución de aplicaciones y servicios. Para completar el despliegue, el servicio LORIOT proporciona una plataforma software para crear y operar una infraestructura del tipo LoRaWAN sin necesidad de desplegar servidores propios para la gestión de los dispositivos loT.

En el caso de querer evaluar estas tecnologías o construir nuevos prototipos basados en ellas, se encuentran disponibles en el mercado diferentes kits y módulos útiles para evaluar las comunicaciones IoT de tipo LPWAN como Sigfox y LoRaWAN, además de poder desarrollar en un breve periodo de tiempo nuevos casos de aplicación. Unos ejemplos de ello son el kit de evaluación LoRaWAN de Microchip, el módulo LoPy4 de Pycom, Arduino MKRFOX1200 y MKRWAN1300 y Waspmote de Libelium. 
CAPÍTULO 3

PROPUESTA DE ARQUITECTURA IOT MIXTA 



\subsection{Introducción}

Las soluciones actualmente disponibles en el ámbito de la telelectura que hacen uso de comunicaciones específicas para loT, están supeditadas a las capacidades de un determinado tipo de red y a su modelo de negocio asociado [Vejlgaard et al., 2017]. Así mismo, los dispositivos finales a ubicar en las viviendas de los abonados, llevan a cabo la función de transmisión de la información de consumo pero no suelen tener capacidad de telecontrol.

Se hace necesaria una arquitectura de red mixta, que combine diferentes soluciones tecnológicas existentes en la actualidad, sea flexible y adaptable a las necesidades particulares según el tipo de despliegue requerido. De la misma manera, se requieren dispositivos capaces de conectar a diferentes tipos de redes loT y de actuar de manera remota sobre un elemento externo como una electroválvula para poder controlar el suministro de servicio al abonado final.

En este capítulo, se lleva a cabo una descripción de la arquitectura de red propuesta así como de los elementos hardware y software que forman parte de la misma y que han sido desarrollados al efecto. También se incluyen los dispositivos que han sido creados de forma específica para el entorno de pruebas y validación en laboratorio.

\subsection{Descripción de la arquitectura propuesta}

La arquitectura de red propuesta, que puede verse esquematizada en la Figura 3.1, combina un nivel de acceso múltiple para la información suministrada por los objetos loT. Es factible recabar datos de dispositivos operando tanto a través de la red Sigfox con cobertura internacional, como a través de posibles redes LoRaWAN ya establecidas o propias desplegadas al efecto, como incluso también de dispositivos ubicados en localizaciones sin cobertura de ninguna de las redes anteriores. En este caso, el enlace se efectúa vía dispositivos intermediarios de desarrollo propio, los cuales facilitan la conectividad utilizando tecnología radio LoRa específica para loT sin sacrificar así autonomía ni alcance.

Las estaciones base desplegadas a lo largo de una zona de cobertura permiten el intercambio de información con los objetos vía un enlace radio inalámbrico específico para loT. No llevan a cabo ningún procesado de los datos, realizan la función de gateway hacía una red IP backhaul que las enlaza con el servidor de red. Se distinguen dos tipos de estaciones base, las de la red Sigfox gestionadas por el mismo operador y las de gestión propia que generan áreas de cobertura mediante tecnología LoRaWAN.

El servidor de red o Core Network lleva a cabo la función de gestión de dispositivos. Deben registrarse en él aquellos nuevos dispositivos que quieran comunicar a través de la red. También maneja la autentificación y el tráfico de los mismos. En función de la calidad del enlace puede solicitar a los dispositivos reajustes en la velocidad de transmisión y el ancho de banda. Al respecto de las tramas recibidas, verifica su integridad y descarta duplicados. Se encarga también de la gestión de gateways, manteniendo una base de datos con los registrados en esa red y seleccionando el más adecuado para transmitir la información de bajada en función del nivel de señal con el que han recibido al dispositivo loT. En esta arquitectura mixta, coexisten dos servidores de red. El primero de ellos gestiona el tráfico de las estaciones base propias LoRaWAN, el segundo de ellos, integrado en la infraestructura del operador Sigfox, gestiona las estaciones base de este tipo y suministra la carga útil recibida de los objetos. Por último, se lleva 
a cabo un reenvío de los datos recibidos al loT SaaS (Software as a Service) vía el broadband link.

El loT SaaS lleva a cabo la gestión de la base de datos que contiene los mensajes recibidos de todos los objetos conectados. Puede utilizar para ello técnicas de 'Big Data' y también puede estar dotado de técnicas de 'Business Intelligence' para el procesado de dichos datos. Además, contiene los interfaces de usuario y administración de forma 'responsive' para permitir que sean accedidos tanto por equipos de escritorio como dispositivos móviles.

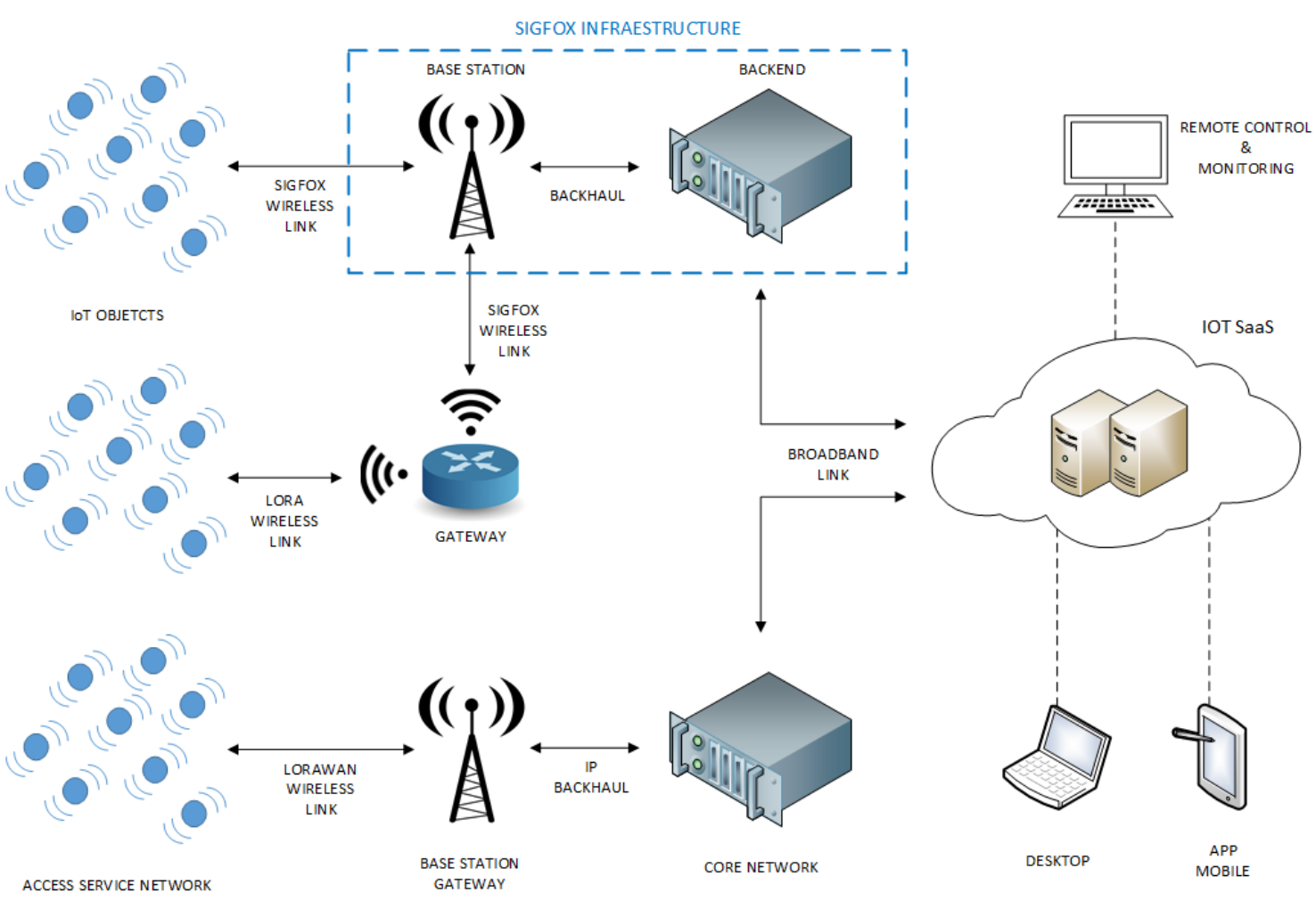

Figura 3.1. Diagrama de la arquitectura de red propuesta.

\subsection{Implementación hardware}

Se detallan a continuación los diversos desarrollos hardware que se han llevado a cabo de dispositivos propios integrantes de la arquitectura propuesta.

\subsubsection{Dispositivo de telemedida y telecontrol con Waspmote}

\subsubsection{Descripción}

El dispositivo aquí descrito, que puede verse en la Figura 3.2, tiene como finalidad el recabar datos de consumo de un contador residencial y enviarlos a una localización remota a través de un enlace radio específico para loT. También es capaz de recibir órdenes de telemando para la actuación sobre una electroválvula y de recibir nuevos parámetros de configuración de la operación. La alimentación proviene de una batería interna de lón-Litio de $6600 \mathrm{mAh}$ de capacidad. 
Los datos de consumo se adquieren bien de un contador preparado para tele-lectura y por tanto con salida de impulsos eléctricos, bien a través de una interface externa de lectura.

Haciendo uso del mismo hardware y en función del firmware que se le programe, el dispositivo es capaz de enviar los datos de consumo a través de la red Sigfox, a través de una red LoRaWAN o mediante LoRa hacia un dispositivo de enlace para zonas de sombra. Se encuentran integradas una radio Sigfox y una radio LoRa/LoRaWAN que pueden actuar simultáneamente. Se utiliza la banda ISM de $868 \mathrm{MHz}$ para la comunicación Sigfox y en el caso de LoRa / LoRAWAN es posible emplear tanto la de $868 \mathrm{MHz}$ como la de $434 \mathrm{MHz}$ mediante antenas independientes.

Para la actuación sobre una electroválvula u otro dispositivo externo similar se dispone de una salida libre de potencial. Como característica a destacar, es capaz de mantener el estado seleccionado sin que ello suponga un consumo de energía continuo en ninguno ellos, bien sea el caso de circuito cerrado o abierto.

La operativa del dispositivo consiste en mantenerse en un estado de bajo consumo hasta que se recibe la señal de impulsos del contador o se cumple el intervalo de tiempo definido para enviar datos. En el caso del impulso del contador, este se contabiliza, se almacena en memoria no volátil y se vuelve al estado de bajo consumo. En caso de haber llegado el momento de enviar datos, se conforma la trama según el protocolo establecido, se inicializa la radio correspondiente y se procede a la transmisión. Si se reciben datos de actuación o de configuración en una trama de bajada, estos se procesan antes de que el dispositivo pase de nuevo al estado de bajo consumo de energía. Este estado permite ampliar la autonomía por encima de un año.

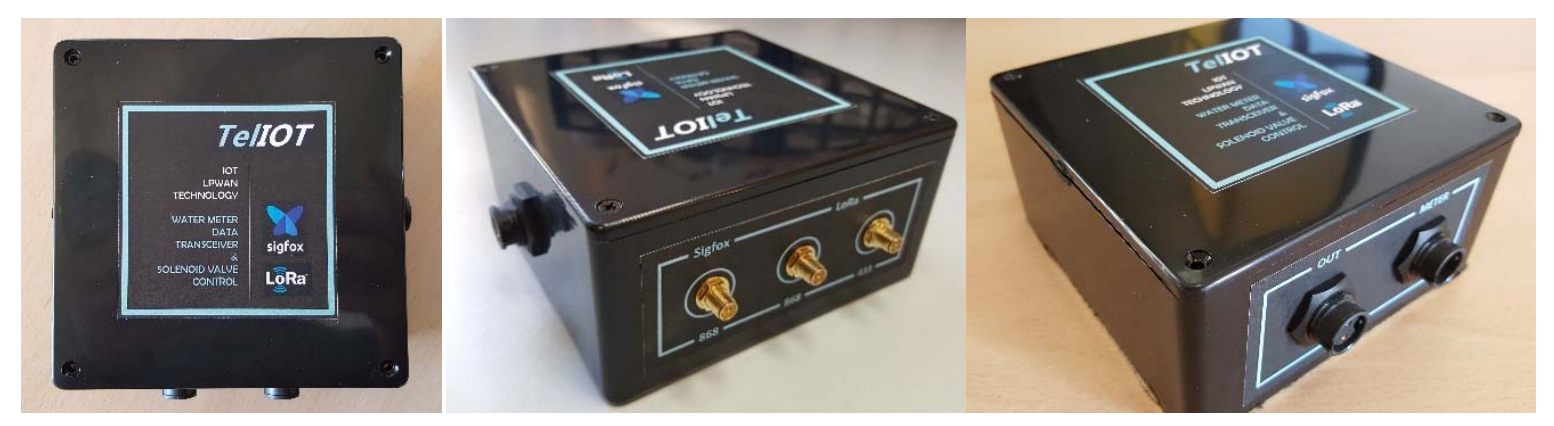

Figura 3.2. Vista exterior del dispositivo.

\subsubsection{Desarrollo hardware}

El equipo consta de una placa base donde se ubican el microcontrolador ATmega1281, la memoria no volátil, el reloj en tiempo real RTC, un regulador de tensión y un controlador de carga de la batería. Esta placa principal sirve de soporte físico e interconexión eléctrica para dos módulos de comunicaciones Sigfox y LoRaWAN, así como para un módulo de expansión propio de esta aplicación para la adquisición de la señal del contador y la actuación sobre una electroválvula. Se basa en la placa microcontrolada de propósito general Waspmote de Libelium [Libelium, 2018] y los módulos de comunicaciones compatibles con ella en formato XBee.

Al disponer la placa principal de un sólo slot de ampliación y ocuparse con el módulo Sigfox, se hace necesario una placa adaptadora intermedia que proporciona un segundo slot XBee para la radio LoRaWAN a partir del puerto GSM/GPS. Ambas radios incluyen un conector de tipo SMA 
para antena externa. Con el fin de situar las antenas en el exterior de la carcasa y hacer más fácil su intercambio, se hace uso de latiguillos de cable coaxial con conector de panel en un extremo. Hay posibilidad de acoplar tres antenas simultáneamente, una para Sigfox en $868 \mathrm{MHz}$ y dos para LoRa en las bandas de 868 y $434 \mathrm{MHz}$. Esto nos va a permitir evaluar y comparar el rendimiento de LoRa en la banda de $434 \mathrm{MHz}$ de disponibilidad menos frecuente que la de 868 $\mathrm{MHz}$ en dispositivos comerciales.

La conexión USB de la placa principal también se ha dispuesto exteriormente usando un conector de panel de tipo Micro-USB. Mediante este puede tanto alimentarse el dispositivo externamente, como recargar la batería interna, como realizar actualizaciones de firmware. La batería incorporada de tecnología lón-Litio permite que el dispositivo opere de manera autónoma sin requerir alimentación externa. El conjunto se encuentra protegido mediante una carcasa de material $A B S$ en cuyos laterales se sitúan los diferentes conectores para las antenas, el suministro eléctrico y la actualización de la programación.

Se consideró como posible solución para esta aplicación el empleo de la placa de expansión Events Board versión 3.0 creada por el mismo fabricante de la placa base y que pode verse en la Figura 3.3. Esto eliminaría la necesidad de diseñar y fabricar una placa de expansión propia, además de poder utilizar en el software la librería también suministrada.

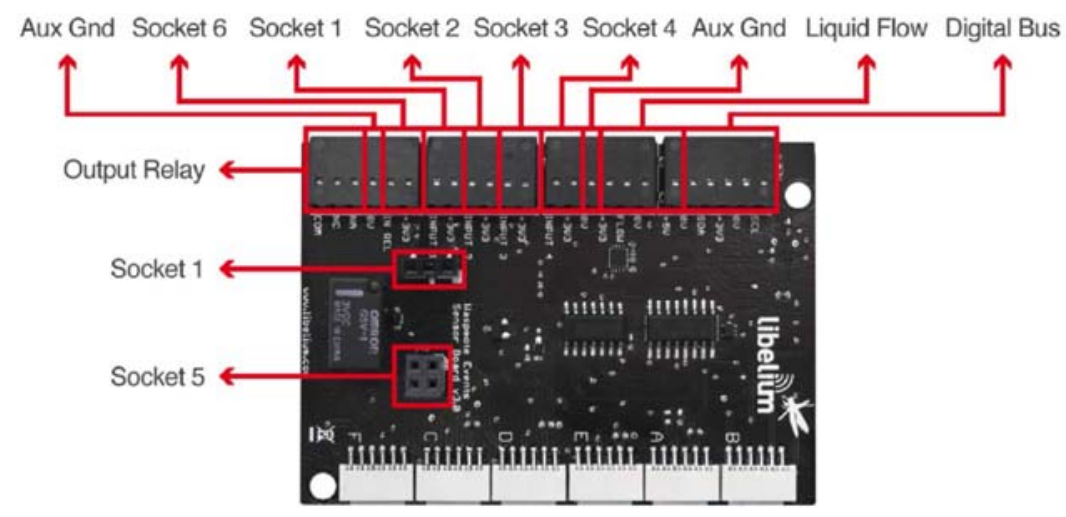

Figura 3.3. Placa de expansión Events Board de Libelium.

La Events Board permite la conexión simultánea de hasta 5 sensores de diferentes tipos. Una de las entradas, denominada relé, admite la conexión de un dispositivo cuya salida libre de potencial cambie entre el estado abierto y cerrado, como podría ser el caso de la interface de lectura de un contador. Esta entrada puede generar una interrupción software cuando detecta un cambio a nivel lógico alto. Para controlar otros dispositivos dispone de una salida libre de potencial a través de un relé incluido que puede abrir o cerrar un circuito eléctrico externo. Esto podría utilizarse para controlar la electroválvula que controlase el suministro de servicio al abonado.

Los inconvenientes detectados, han sido:

- Elevado precio debido en parte por tratarse de una placa multipropósito con más entradas que las necesarias en esta aplicación.

- La entrada para contactos no aporta nada respecto a utilizar la entrada digital disponible en el conector de expansión de la placa base, que además dispone de capacidad de interrupción. 
- Limitadas características eléctricas del circuito de control del relé (24 Vdc / 1 A). No se podría manejar directamente una electroválvula de 220 Vac y requeriría otro relé o contactor intermedio).

- Mientras el relé esté activo, se consume energía de manera continua.

Por estas razones se concluye no utilizar esta opción y pasar a diseñar una placa de expansión específica para esta aplicación. Esta solución permite:

- Disponer de una entrada que a la recepción de un impulso despierte al procesador del estado de bajo consumo.

- Capacidad de controlar cargas de mayor corriente en DC y posibilidad de que sean de 220 VAC.

- Sin consumo de energía continuo una vez establecido un nuevo estado.

La placa de expansión desarrollada específicamente para esta aplicación incluye la circuitería necesaria para la lectura de pulsos del contador y para la actuación sobre electroválvula. Se interconecta con la principal a través del interface estándar para placas de expansión. Incluye un relé bi-estable y circuitería amplificadora de corriente para poder manejar este. La señal procedente del contador de consumo del abonado así como la de actuación sobre la electroválvula, se encuentran disponibles en el exterior de la carcasa mediante conectores roscados de tipo industrial.

Para la adquisición de la señal de pulsos proveniente del contador se utiliza la entrada digital RXD1 que puede generar una interrupción al producirse un cambio de nivel. Se limita la corriente con una resistencia y se fija el nivel lógico bajo por defecto mediante una resistencia de mayor valor conectada a masa. Para su correcto funcionamiento es necesario también fijar de manera permanente un nivel lógico alto en la entrada DIG2. Un condensador de desacoplo permite eliminar falsos disparos por ruido de alta frecuencia. La señal RXD1 genera una interrupción al recibir un flanco ascendente, por lo que la salida libre de potencial del contador cierra un circuito con tensión proveniente de la alimentación de 3.3V.

Para poder actuar sobre electroválvulas externas tanto de baja tensión como de media tensión, se ha previsto una salida libre de potencial mediante los contactos de un relé. Al consumir la bobina del dicho relé una corriente superior a la que pueden proporcionar las salidas digitales, se hace necesario incluir circuitería que haga la función de amplificador de corriente. $Y$ al tratarse de un relé bi-estable con dos bobinas, es necesario duplicar esta circuitería. Para el amplificador se utilizan transistores NPN trabajando en conmutación que a través de su colector cierran a masa el circuito proveniente de las bobinas del relé. Estas tienen conectado su extremo común a la salida de tensión regulada a $5 \mathrm{~V}$ de la placa principal. El utilizar $5 \mathrm{~V}$ en lugar de $3.3 \mathrm{~V}$ se debe a que el consumo de un relé a esta tensión es inferior al de uno similar de $3 \mathrm{~V}$, en concreto $120 \mathrm{~mA}$ frente a $240 \mathrm{~mA}$, y queda por debajo del límite de máxima corriente de $200 \mathrm{~mA}$ suministrable por la placa base.

Para cambiar el estado del relé y por tanto conmutar su circuito de salida, se genera un pulso de nivel alto y duración 30ms a través de las salidas digitales DIG1 y DIG3 según el estado en que se quiera dejar la salida. Aunque la salida del relé puede actuar como un conmutador, en esta aplicación se utiliza sólo como un interruptor que cierra la circulación de corriente de un circuito externo que puede ser tanto de baja tensión DC (electroválvulas de $12024 \mathrm{~V}$ ) como de media tensión AC (220 V). 
Un diagrama de bloques del dispositivo se encuentra representado en la Figura 3.4.

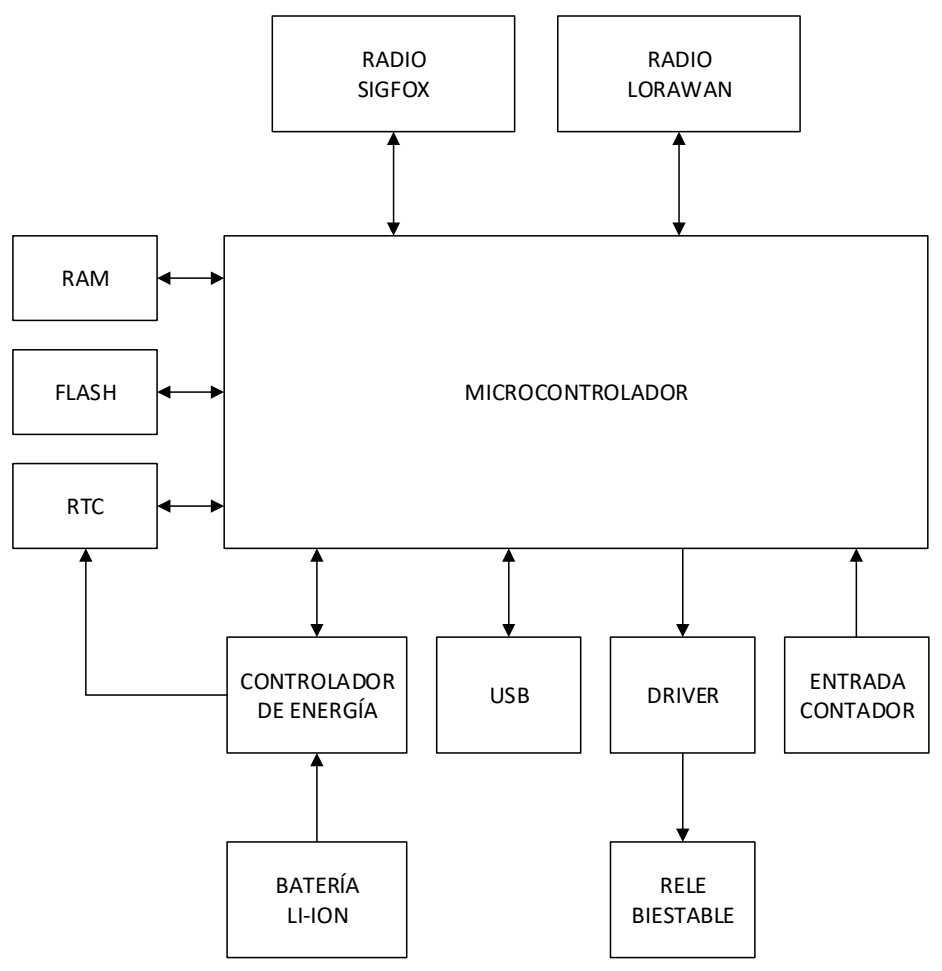

Figura 3.4. Diagrama de bloques del dispositivo.

El diagrama de conexionado eléctrico puede consultarse en la siguiente Figura 3.5.
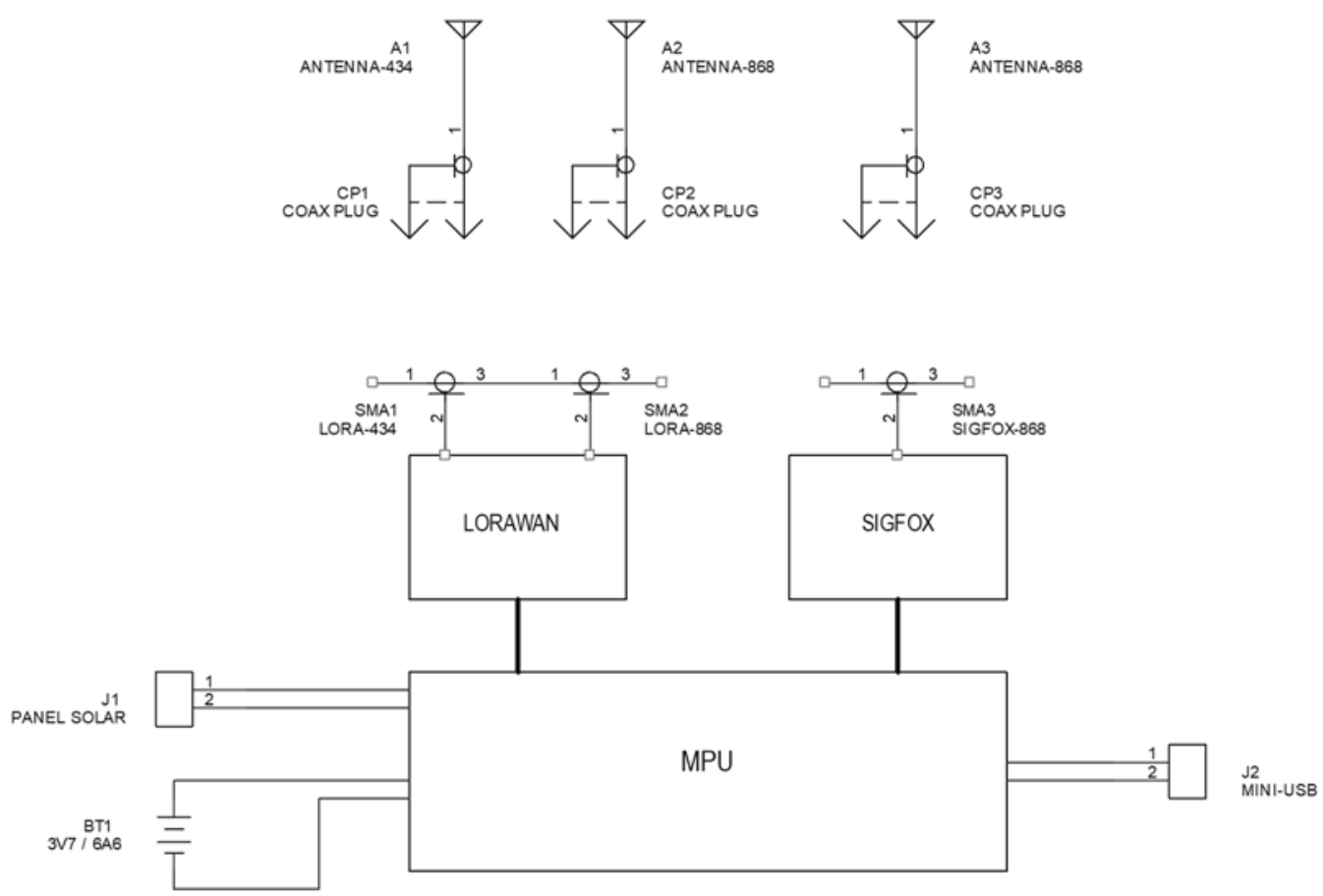

Figura 3.5. Diagrama de conexionado del dispositivo.

El esquemático detallado a nivel de componentes electrónicos puede verse en la Figura 3.6. 


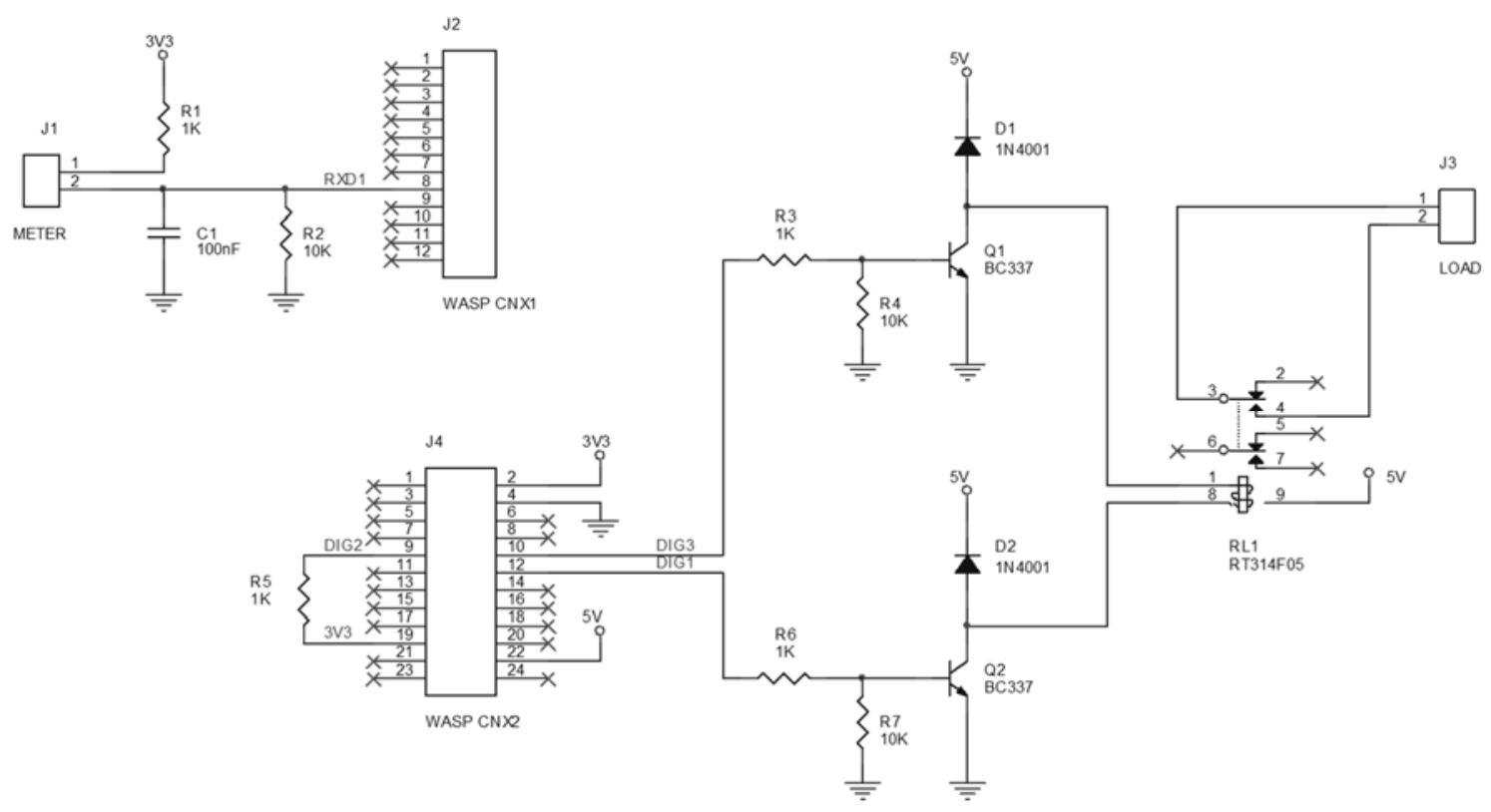

Figura 3.6. Esquemático electrónico.

Los cálculos eléctricos han sido los siguientes. El relé seleccionado es un bi-estable con 2 bobinas de 3V (RT314F03 de OEG-TE Connectivity). Se procede a calcular el driver de corriente para su excitación a partir de las salidas digitales de $3 \mathrm{~V}$ y $40 \mathrm{~mA}$.

$$
\begin{aligned}
& \mathrm{R}_{\text {bob typ }}=15 \Omega \rightarrow \mathrm{I}_{\mathrm{ctyp}}=\frac{\mathrm{V}_{\mathrm{c}}}{\mathrm{R}_{\text {bob typ }}}=\frac{3}{15}=0.2 \mathrm{~A} \\
& \left.\mathrm{R}_{\text {bob max }}=16.5 \Omega \rightarrow \mathrm{I}_{\mathrm{c} \min }=\frac{\mathrm{V}_{\mathrm{c} \min }}{\mathrm{R}_{\mathrm{bob} \max }}=\frac{3}{16.5}=0.182 \mathrm{~A}\right\} \Rightarrow \mathrm{I}_{\mathrm{c}}>0.244 \mathrm{~A} \\
& \left.\mathrm{R}_{\mathrm{bob} \min }=13.5 \Omega \rightarrow \mathrm{I}_{\mathrm{c} \max }=\frac{\mathrm{V}_{\mathrm{c} \max }}{\mathrm{R}_{\mathrm{bob} \min }}=\frac{3.3}{13.5}=0.244 \mathrm{~A}\right) \\
& \left.\begin{array}{r}
\mathrm{I}_{\mathrm{c}}>0.244 \mathrm{~A} \\
\mathrm{NPN} \\
\mathrm{I}_{\mathrm{BC} 337}=0.8 \mathrm{~A}
\end{array}\right\} \Rightarrow \mathrm{T}_{1} \text { seleccionado }=\mathrm{BC} 337 \\
& \left.\begin{array}{c}
\mathrm{V}_{\mathrm{BE}}\left(\mathrm{BC} 337, \mathrm{I}_{\mathrm{C}}=244 \mathrm{~mA}\right)=1 \mathrm{~V} \\
\mathrm{I}_{\mathrm{B}}=\frac{\mathrm{I}_{\mathrm{C}}}{\mathrm{hFE}(300 \mathrm{~mA})}=\frac{244 \mathrm{~mA}}{100}=2.44 \mathrm{~mA}
\end{array}\right\} \mathrm{R}_{\mathrm{B}}=\frac{\mathrm{V}_{\mathrm{cc}}-\mathrm{V}_{\mathrm{BE}}}{\mathrm{I}_{\mathrm{B}}}=\frac{3-0.7}{2.44 \mathrm{~mA}}=819.67 \Omega \simeq 1 \mathrm{k} \Omega \\
& \mathrm{R}_{\mathrm{B}-\mathrm{GND}}=10 \cdot \mathrm{R}_{\mathrm{B}}=10 \mathrm{k} \Omega
\end{aligned}
$$

Cálculo de las resistencias limitadoras de corriente en las entradas digitales.

$$
\begin{aligned}
& \mathrm{R}_{\mathrm{I}}=\frac{\mathrm{V}_{\mathrm{CC}}}{\mathrm{I}_{\mathrm{Imax}}}=\frac{3.3 \mathrm{~V}}{3.3 \mathrm{~mA}}=1 \mathrm{k} \Omega \\
& \mathrm{R}_{\mathrm{I}-\mathrm{GND}}=10 \cdot \mathrm{R}_{\mathrm{I}}=10 \mathrm{~K} \Omega
\end{aligned}
$$


Para un rápido prototipado y verificación de la solución, se ha utilizado una placa de circuito pretaladrada en la que se han ubicado los componentes discretos y realizado las conexiones a una sola cara como puede verse en la Figura 3.7.
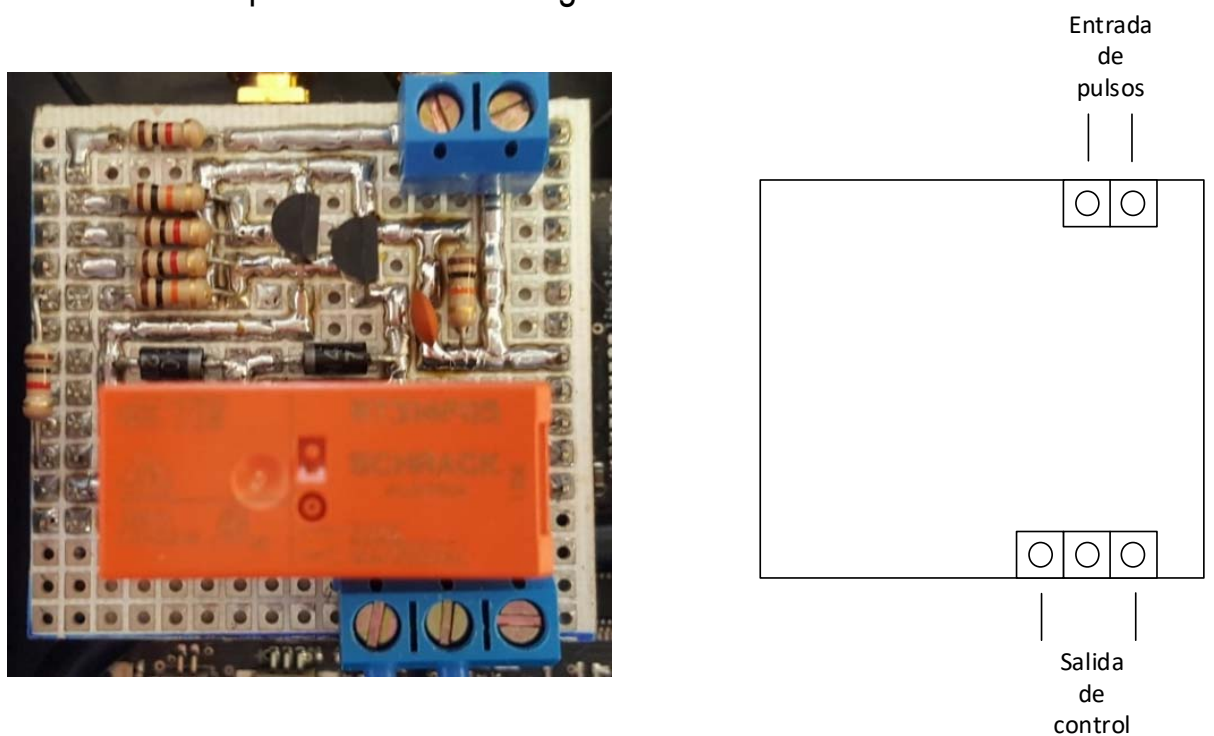

Figura 3.7. Placa de expansión propia.

El conjunto resultante de placa base con placa de expansión personalizada para la aplicación puede verse en la Figura 3.8.

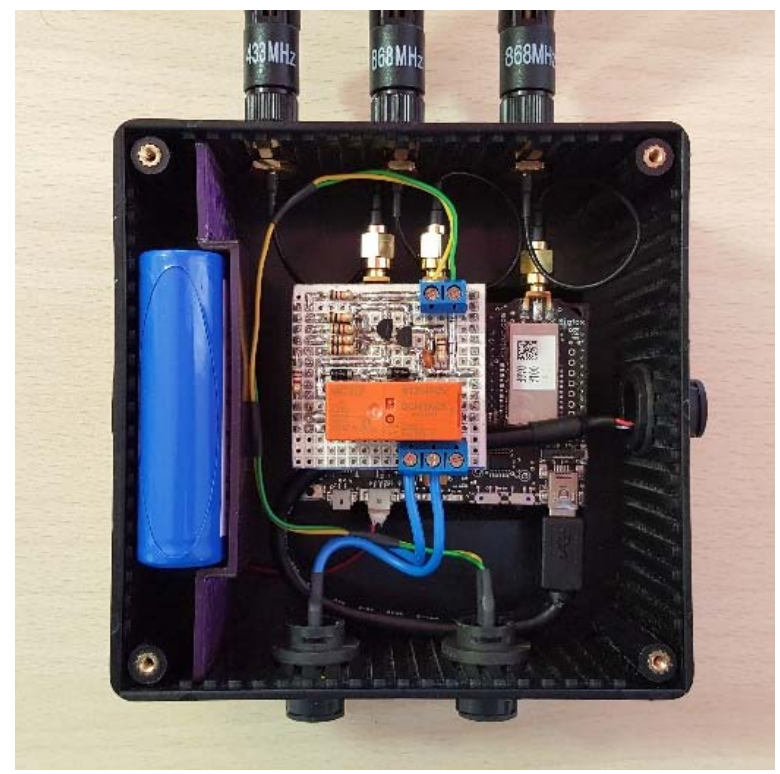

Figura 3.8. Vista interior del dispositivo.

La posición de los micro-interruptores de configuración que están ubicados en la placa base tal y como puede verse en la Figura 3.9, debe ser la siguiente para esta aplicación:

- Micro-interruptor superior izquierdo

Función: POWER (Alimentación del circuito)

Posición: ON / Hacia LEDs (izquierda mirando desde arriba) 
- Micro-interruptor inferior

Función: HIBERNATE (Habilita la posibilidad de hibernar)

Posición: OFF / Hacia LEDs (izquierda mirando desde arriba)

- Micro-interruptor superior derecho (versión de placa a partir de 1.5)

Función: WATCHDOG (Habilita el perro guardián ante posibles cuelgues)

Posición: OFF - Hacia LEDs (izquierda mirando desde arriba)
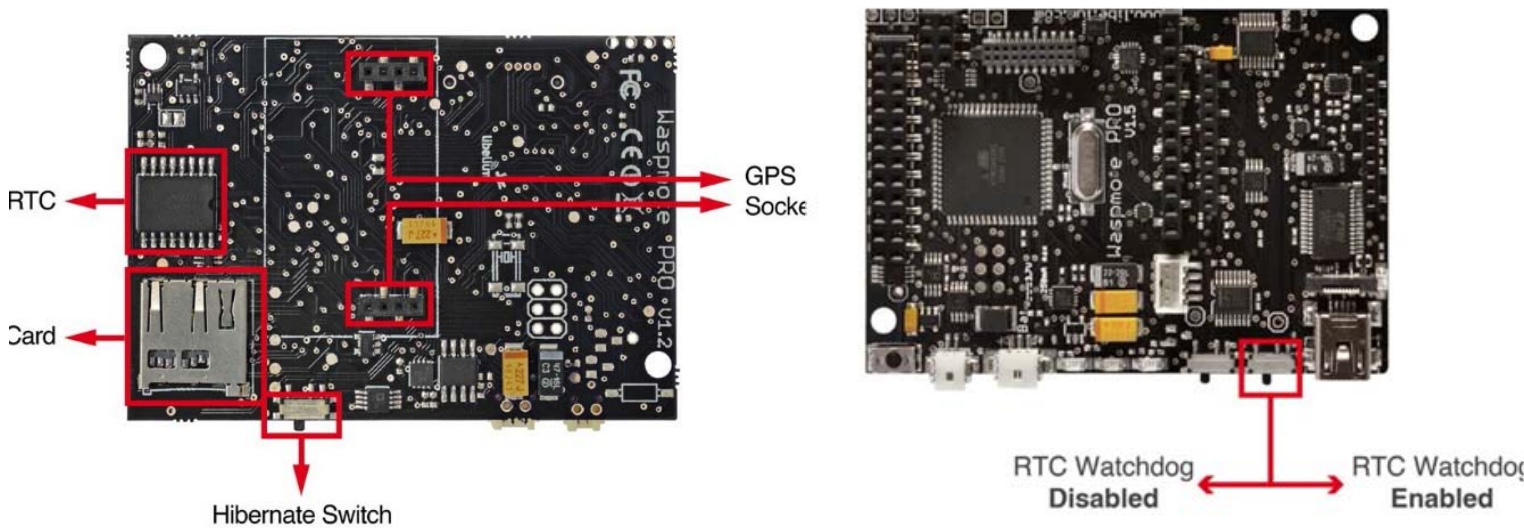

Figura 3.9. Micro-interruptores de configuración de la placa Waspmote.

Los conectores externos disponibles incluyendo su función y características de la señal que manejan se encuentran listados en la Tabla 3.1.

\begin{tabular}{|l|l|l|}
\hline \multirow{4}{*}{ USB } & Tipo & Micro-USB tipo A hembra \\
\cline { 2 - 3 } & Función & Actualizaciónes firmware / Carga de batería \\
\cline { 2 - 3 } & Tensión & $5 \mathrm{~V}$ \\
\cline { 2 - 3 } & Corriente carga & $100 \mathrm{~mA}$ \\
\cline { 2 - 3 } & Tiempo carga & $66 \mathrm{~h}(0-100 \%)$ \\
\hline \multirow{4}{*}{ Contador } & Tipo & Industrial roscado de 16mm con 2 pines macho \\
\cline { 2 - 3 } & Función & $\begin{array}{l}\text { Entrada de señal procedente del contador sin } \\
\text { polarización }\end{array}$ \\
\cline { 2 - 3 } & Rango tensión & $3.3 \mathrm{~V}$ \\
\cline { 2 - 3 } & Corriente & $1 \mathrm{~mA}$ \\
\hline \multirow{4}{*}{ Salida } & Tipo & $\begin{array}{l}\text { Industrial roscado de 16mm con 2 pines } \\
\text { hembra }\end{array}$ \\
\cline { 2 - 3 } & Función & $\begin{array}{l}\text { Contacto libre de potencial para control de } \\
\text { electroválvula }\end{array}$ \\
\cline { 2 - 3 } & Rango tensión & 250 Vac \\
\cline { 2 - 3 } & Corriente carga & $10 \mathrm{~A}$ \\
\hline \multirow{5}{*}{$\begin{array}{l}\text { Antena } \\
\text { Sigfox 868 }\end{array}$} & Tipo & SMA hembra invertido \\
\cline { 2 - 3 } & Frecuencia & $868 \mathrm{MHz}$ \\
\cline { 2 - 3 } & Potencia & $14 \mathrm{dBm}$ \\
\hline
\end{tabular}




\begin{tabular}{|l|l|l|}
\hline \multirow{3}{*}{$\begin{array}{l}\text { Antena Lora } \\
\text { n }\end{array}$} & Tipo & SMA hembra invertido \\
\cline { 2 - 3 } & Frecuencia & $868 \mathrm{MHz}$ \\
\cline { 2 - 3 } & Potencia & $14 \mathrm{dBm}$ \\
\hline \multirow{2}{*}{$\begin{array}{l}\text { Antena Lora } \\
433\end{array}$} & Tipo & SMA hembra invertido \\
\cline { 2 - 3 } & Frecuencia & $434 \mathrm{MHz}$ \\
\cline { 2 - 3 } & Potencia & $14 \mathrm{dBm}$ \\
\hline
\end{tabular}

Tabla 3.1. Conectores externos del dispositivo.

El proceso de carga de la batería interna puede llevarse a cabo mediante alimentador externo a través del conector mini-USB o mediante un panel solar a través del conector específico para ello.

En el caso de alimentador externo, este ha de tener las siguientes características:

- Tensión constante de $5 \mathrm{~V}$

- Corriente mínima de $500 \mathrm{~mA}$

- Conector de salida macho tipo mini-USB

Para iniciar la carga basta con conectar un alimentador al conector correspondiente. Si se quiere verificar, un LED rojo se ilumina en el borde de la placa base y se apaga cuando la carga finaliza. En función del estado de descarga de la batería, recuperar la carga completa de la misma puede tardar hasta 66 horas.

\subsubsection{Desarrollo software}

La operativa del software embebido en el microcontrolador del dispositivo se describe a continuación. Tras el encendido, se procede a inicializar el hardware con la configuración adecuada para esta aplicación. Se activa el regulador de tensión de $3 \mathrm{~V}$ y se desactiva el de $5 \mathrm{~V}$ para ahorrar energía. Se configuran los pines de entrada y salida digitales según la función requerida, se habilita la interrupción externa y se configura la interrupción del reloj en tiempo real con el intervalo entre envíos por defecto.

En cada ciclo de ejecución se procede a realizar las siguientes tareas. En primer lugar se obtienen la hora del reloj en tiempo real RTC y la última lectura del contador almacenada en la EEPROM. Se distinguen dos casos en función de la causa de inicio de ejecución. Si se trata de la primera ejecución después del encendido o se ha despertado por interrupción del RTC al cumplirse el tiempo programado para envío, se procede a la transmisión de una trama de información por la correspondiente radio loT. Al compilar el firmware puede seleccionarse el uso de Sigfox, LoRaWAN o LoRa. Esto hará que se utilice un módulo radio u otro y sus correspondientes funciones. Si en el envío se ha solicitado y se recibe una trama de bajada con nueva configuración, se procede a actualizar los parámetros correspondientes. En el caso de Sigfox esto sucede sólo una vez al día por limitaciones de la red. En función del intervalo entre envíos configurado, se calcula la hora a programar en el RTC para el siguiente despertar y transmisión, descontando el tiempo consumido en este envío. Por último se pasa a modo de sueño profundo con bajo consumo de energía. Si se ha despertado por generarse una interrupción externa al recibirse un pulso del contador, se procede a cuantificarlo y a guardar el nuevo valor en la memoria no volátil de tipo EEPROM. A continuación se activa el modo de 
sueño profundo con bajo consumo de energía habilitando el despertar de nuevo por RTC si no se produce antes otra interrupción por recepción de pulsos del contador.

Los módulos radio permanecen en modo de reposo con un consumo mínimo de energía hasta que es requerido su uso para la transmisión de datos. Es en este momento cuando son inicializados y al finalizar la actividad, devueltos a modo de bajo consumo.

El anterior proceso se repite continuamente. El flujograma puede consultarse en la Figura 3.10.
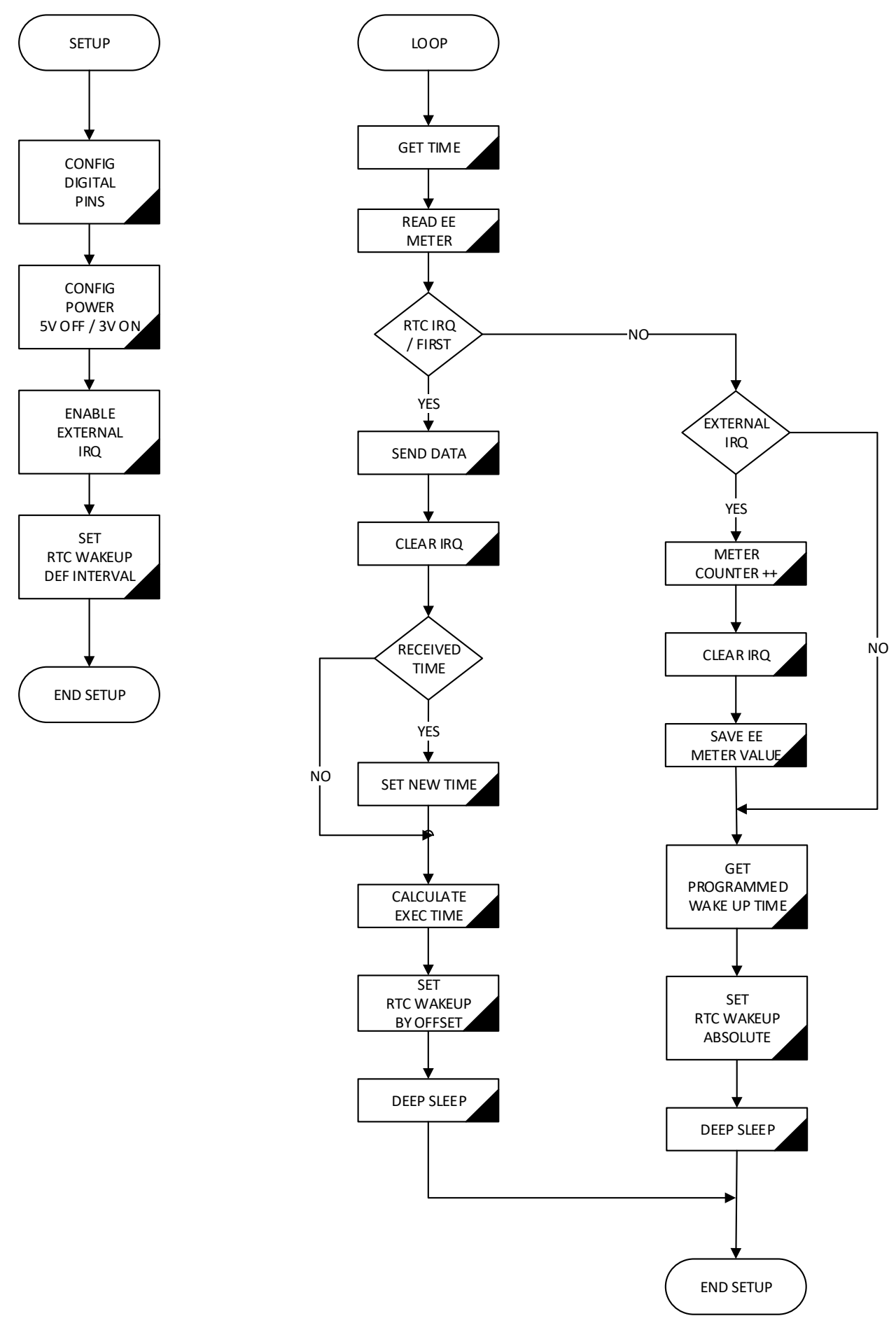

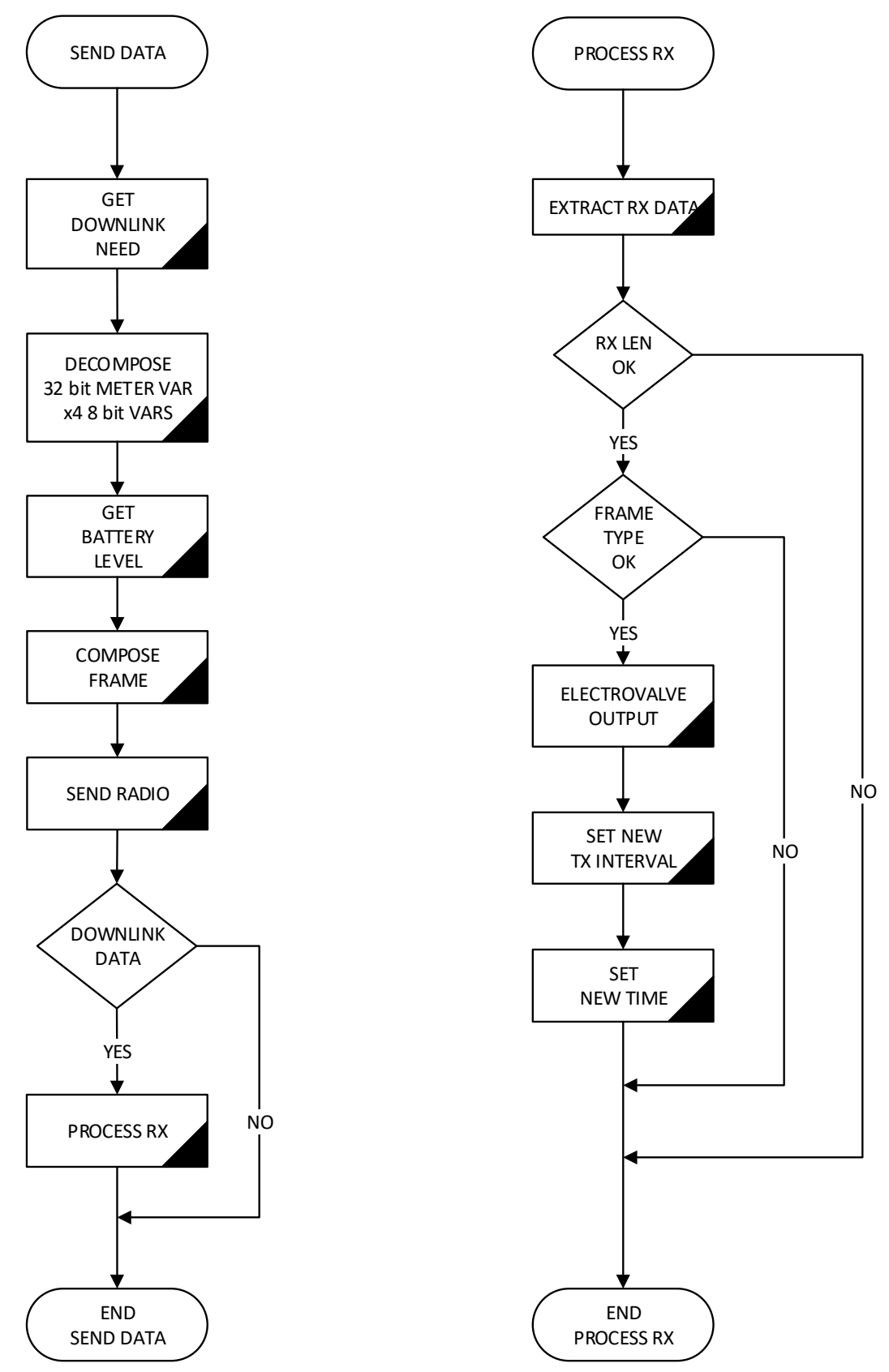

Figura 3.10. Flujograma del firmware del dispositivo. 
Un diagrama de funciones por nivel está representado en la Figura 3.11.

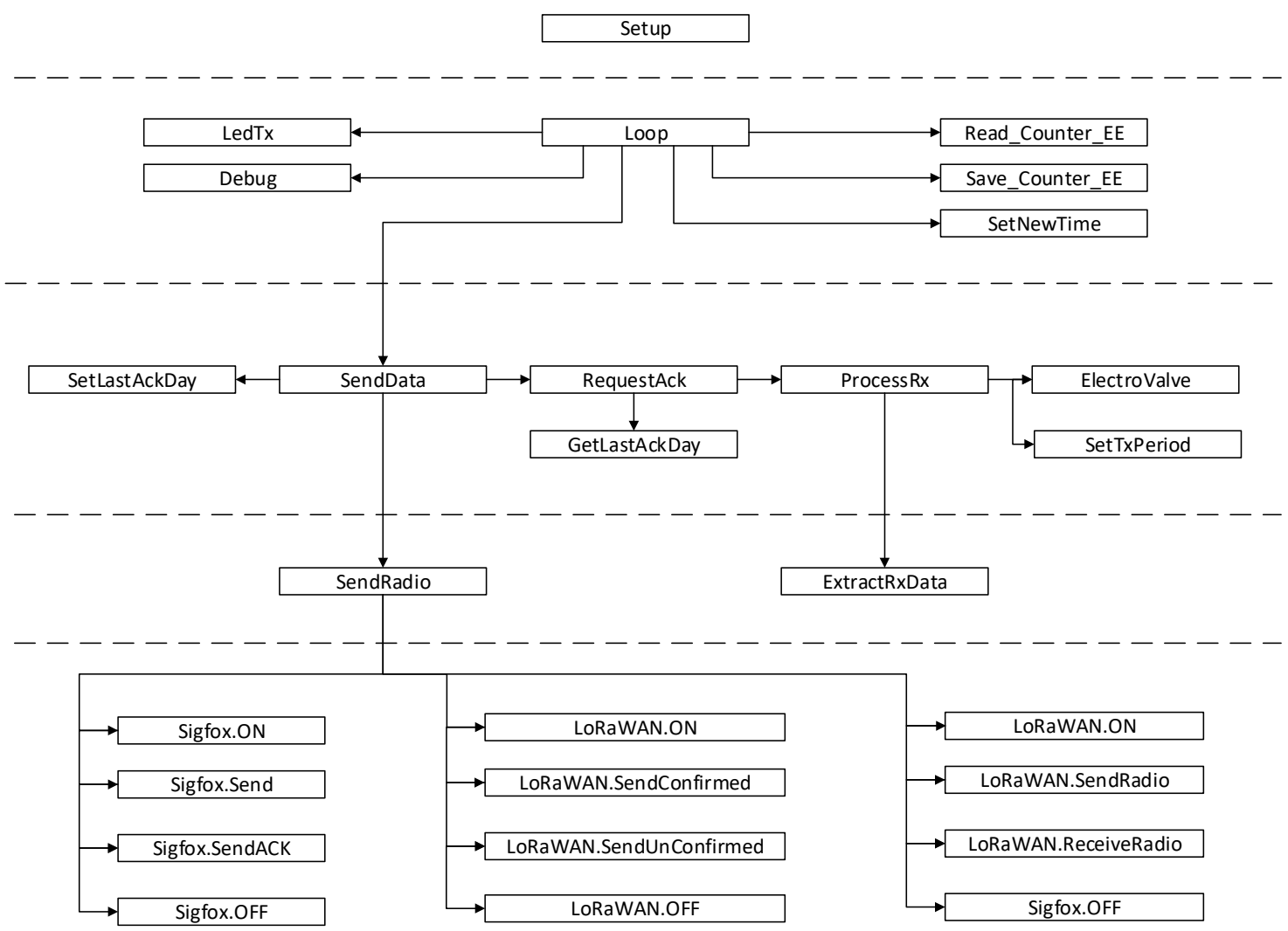

Figura 3.11. Diagrama de funciones por nivel.

Dado que el dispositivo obtiene su energía de una batería interna y con objeto de maximizar la duración de esta, en ausencia de actividad se pasa al modo de ahorro de energía con consumo mínimo. Las interrupciones externa y del temporizador sacan al procesador de este modo y se reanuda la operación. Se llevan a cabo las correspondientes tareas y se programa el siguiente despertar con el tiempo configurado entre transmisiones antes de volver al modo de bajo consumo. El siguiente despertar se calcula restándole al tiempo definido en configuración, el tiempo consumido en la operación de transmisión que puede ser variable según las condiciones de enlace con la red o los tiempos de respuesta de esta cuando se incluye una solicitud de bajada. Un diagrama completo de los tiempos de actividad se encuentra en la Figura 3.12. 


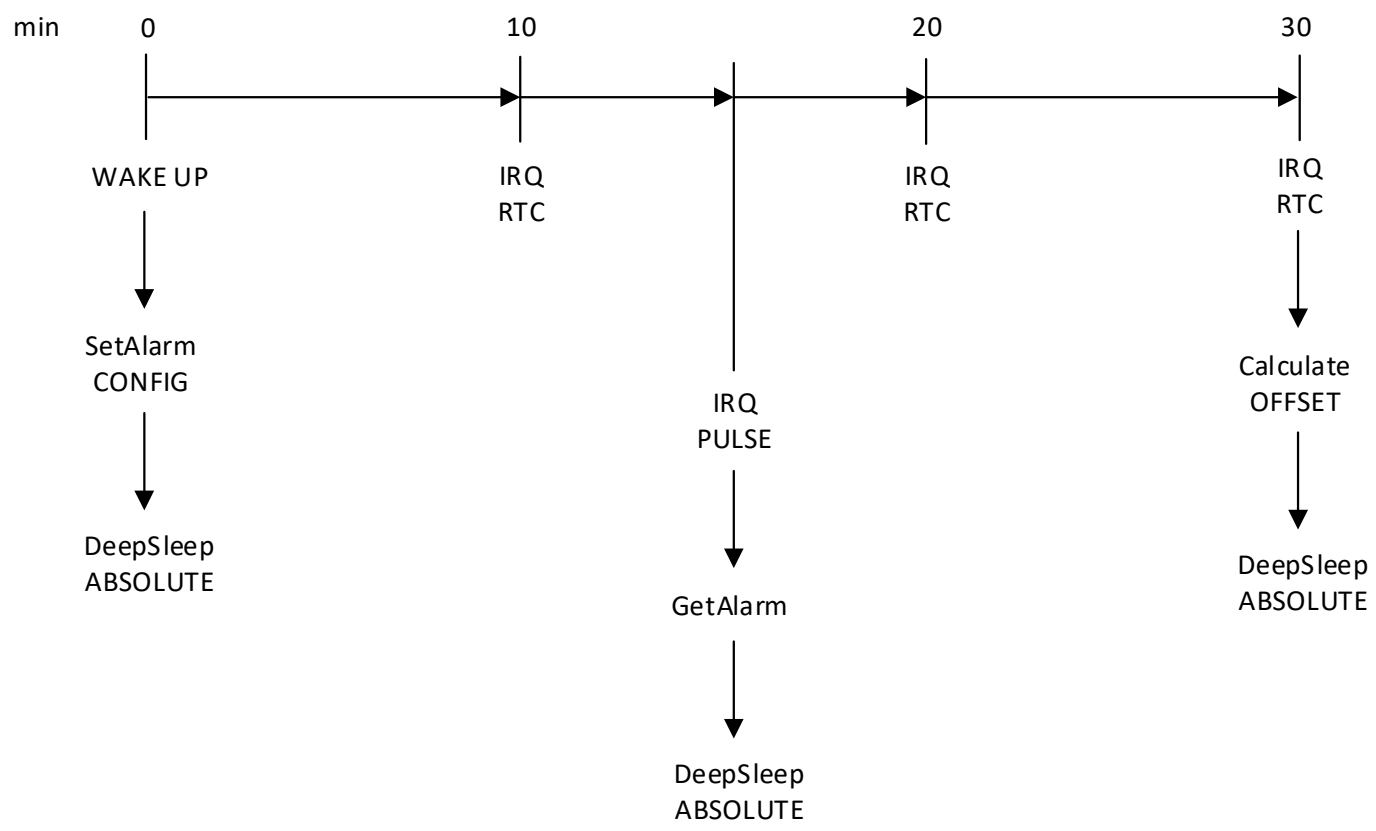

Figura 3.12. Diagrama de intervalos de actividad.

\subsubsection{Dispositivo de telemedida y telecontrol con PyCom}

\subsubsection{Descripción}

El dispositivo aquí descrito consiste en una versión optimizada del diseño basado en Waspmote que, al igual que este, tiene como finalidad el recabar datos de consumo de un contador residencial y enviarlos a una localización remota a través de un enlace radio específico para loT. También es capaz de recibir órdenes de telemando para la actuación sobre una electroválvula y de recibir nuevos parámetros de configuración. Respecto al anterior dispositivo, en esta versión se ha reducido a la mitad el tamaño del dispositivo simplificando el diseño, se ha integrado la antena, se ha minimizado el número de componentes electrónicos, se ha reducido el coste final y se han manteniendo el resto de características. El software embebido se ha escrito desde cero para adaptarlo al nuevo procesador pero mantiene la misma funcionalidad. En la Figura 3.13 se encuentra una vista exterior del dispositivo y una interior en la Figura 3.14.

Los datos de consumo se adquieren bien de un contador preparado para tele-lectura y por tanto con salida de impulsos eléctricos, bien a través de una interface externa de lectura. El dispositivo es capaz de enviar los datos de consumo a través de la red Sigfox, a través de una red LoRaWAN y mediante una conexión LoRa punto a punto, a otro dispositivo intermediario que permite ampliar la cobertura a ubicaciones de sombra. Todo ello en la banda ISM de $868 \mathrm{MHz}$. La alimentación proviene de una batería interna recargable de lón-Litio. Para la actuación sobre una electroválvula u otro dispositivo externo similar, se dispone de una salida libre de potencial.

La operativa del dispositivo consiste en mantenerse en un estado de bajo consumo hasta que se recibe señal del contador o se cumple el intervalo de tiempo para enviar datos. En el caso de la señal del contador en forma de impulso eléctrico, este se contabiliza, se almacena en memoria no volátil y se vuelve a un estado de bajo consumo. En caso de haber llegado el momento de enviar datos, se conforma la trama según el protocolo establecido, se inicializa la radio correspondiente y se procede a la transmisión. Si se reciben datos de actuación o de 
configuración, estos se procesan antes de pasar de nuevo al estado de bajo consumo de energía.
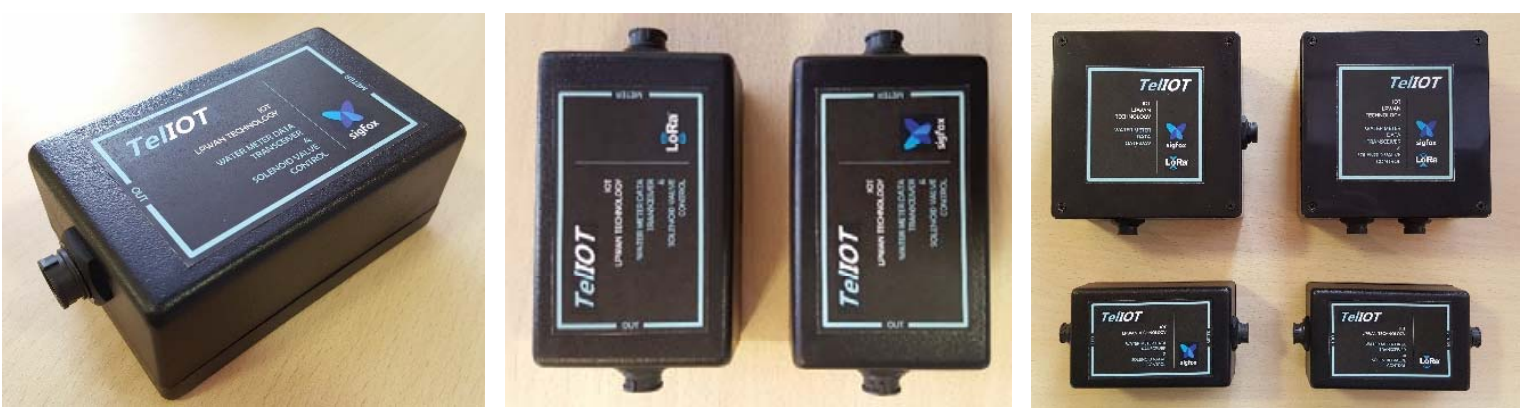

Figura 3.13. Vista exterior del dispositivo.

El equipo consta de una placa electrónica donde se ubican el módulo que integra control y comunicaciones, así como los componentes necesarios para facilitar la lectura de pulsos del contador y para la actuación sobre una electroválvula. La salida, libre de potencial, es capaz de mantener el estado establecido sin un consumo continuo de energía.

En el interior de la carcasa de material ABS se encuentra integrada una antena adhesiva de reducidas dimensiones para la banda ISM de $868 \mathrm{MHz}$. También se ubica una batería de tecnología lón-Litio que permite que el dispositivo opere de manera autónoma durante años sin requerir de alimentación externa. Pasado este periodo la batería podría recargarse sin necesitar sustitución. La conexión externa tanto del contador como de la electroválvula, se llevan a cabo mediante conectores roscados de panel ubicados a ambos lados de la carcasa. Para evitar posibles confusiones, son de géneros opuestos, siendo el de tipo macho para el contador y el de tipo hembra para la electroválvula.
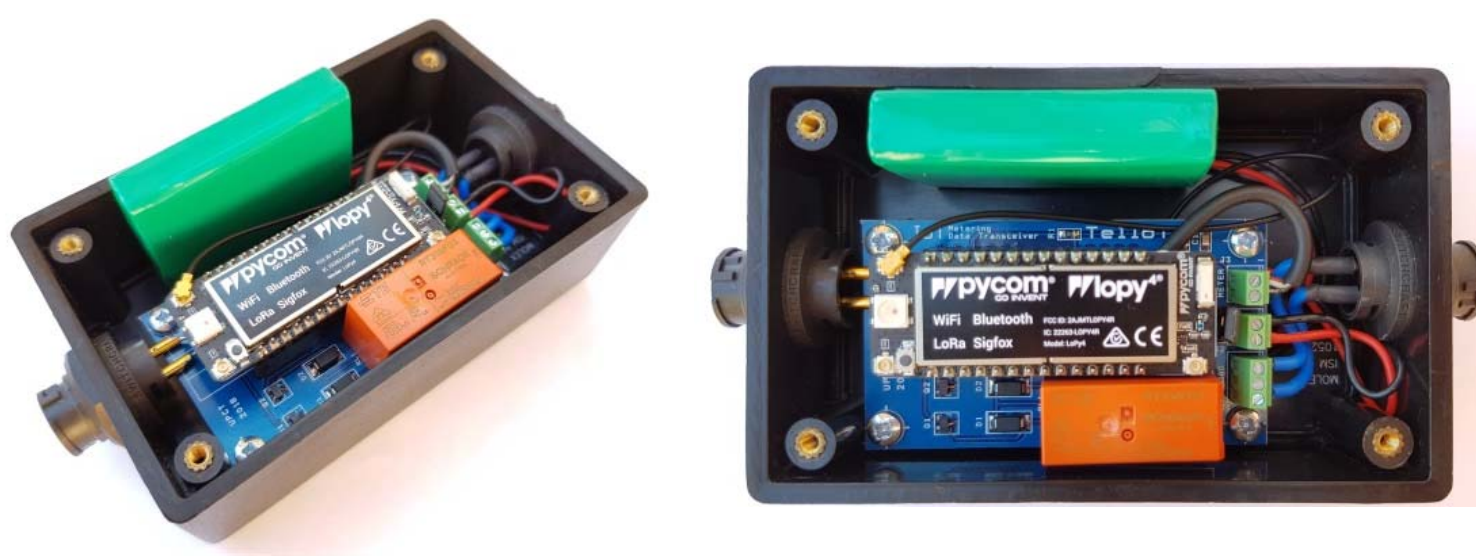

Figura 3.14. Vista interior del dispositivo.

\subsubsection{Desarrollo hardware}

El diseño se basa en el módulo Pycom [Pycom, 2018b] para desarrollo de soluciones loT. A este se le ha añadido la circuitería necesaria para ofrecer la funcionalidad requerida en esta aplicación.

Los módulos Pycom incorporan como procesador el chipset Espressif ESP32 ejecutando un intérprete de MicroPython. Esto ofrece una combinación de potencia, facilidad de uso y 
flexibilidad. Incluyen conectividad Wi-Fi, Bluetooth LE y a una red loT de tipo LPWAN. El modelo seleccionado denominado LoPy4 incluye conectividad a redes Sigfox o LoRaWAN y enlaces punto a punto mediante modulación radio FSK (Frecuency Shift Keying) o LoRa. La antena para Wi-Fi y Bluetooth está integrada en el módulo, no así la de LPWAN para la que se dispone de un conector de tipo UFL. A este se ha conectado una antena flexible y de reducidas dimensiones adecuada a esa banda radio y ubicada en el interior de la carcasa.

Para la adquisición de la señal de pulsos proveniente del contador se utiliza una entrada digital capaz de sacar al procesador del modo de bajo consumo o sueño profundo. La entrada es configurada por software para actuar ante un nivel lógico bajo. Esta señal y masa se llevan al interface de lectura del contador, el cual produce en cada pulso un cierre de circuito por su salida libre de potencial.

Al consumir el relé de salida una corriente superior a la que pueden proporcionar las salidas digitales, se hace necesario incluir circuitería que haga la función de amplificador de corriente. Por tratarse de un relé bi-estable con dos bobinas, es necesario duplicar esta circuitería. Para el amplificador se utilizan transistores NPN trabajando en conmutación y que a través de su colector cierran a masa el circuito proveniente de las bobinas del relé que tienen conectado su extremo común al positivo de la tensión de alimentación. Para cambiar el estado del relé y por tanto conmutar su circuito de salida, se genera un pulso de nivel alto y duración $30 \mathrm{~ms}$ a través de las salidas digitales según el estado en que se quiera dejar la salida. Aunque el relé puede actuar como un conmutador, en esta aplicación se utiliza como un interruptor para cerrar un circuito eléctrico externo del que forma parte la electroválvula. Esta puede ser de baja tensión y corriente continua (12 Vdc o $24 \mathrm{Vdc}$ ) o media tensión alterna (220 Vac).

La alimentación proviene de una batería interna de lón-Litio recargable. El rango de tensión de ésta (3.6 a $4.2 \mathrm{~V}$ ), es compatible con el rango de alimentación admisible por el módulo Pycom $(3.4 \mathrm{~V}-5.5 \mathrm{~V})$. Dado el bajo consumo del diseño, la autonomía puede extenderse durante años en función de la configuración. Cumplido este periodo, la batería puede recargarse sin tener que ser desechada.

Las principales características del controlador PyCom son las siguientes:

Dimensiones y peso

- Dimensiones: $55 \times 20 \times 35 \mathrm{~mm}$

- Peso: $7 \mathrm{~g}$

\section{Energía}

- Rango de voltaje de alimentación: $3.3 \mathrm{~V}-5.5 \mathrm{~V}$

- Salida regulada de $3.3 \mathrm{~V}$ y hasta $400 \mathrm{~mA}$

- Consumo de radio WiFi: 12 mA en modo activo, $5 \mathrm{uA}$ en reposo

- Consumo de radio Sigfox/LoRa: $12 \mathrm{~mA}$ en modo activo, 5 uA en reposo

\section{Procesador}

- Chipset Espressif ESP32

- Sistema on-chip de doble procesador y radio WiFi

- El procesador de red maneja la conectividad WiFi y la pila IP v6

- El procesador principal queda totalmente libre para ejecutar el código de usuario

- Un coprocesador monitoriza las entradas/salidas digitales y canales ADC durante el modo de sueño profundo con solo 25 uA de consumo

- Memoria RAM: $512 \mathrm{kB}$ 
- Memoria Flash: $4 \mathrm{MB}$

- GPIO: Hasta 24

- Aceleración de coma flotante por hardware

- Python multi-hilo

- RTC a 32Khz

Interfaces

- Entradas y salidas digitales: Hasta 24

- Puertos serie UART: 2

- Buses digitales: 2xSPI, I2C, I2S

- Interface para tarjeta micro-SD

- Convertidores analógico-digital: 8XADC 12 bit

- Temporizadores: 4 de 16bits con PWM y captura de la entrada

- Acceso directo a memoria en todos los periféricos

Conectividad WiFi y Bluetooth

- Compatible Bluetooth Low Energy y clásico

- WiFi compatible $802.11 \mathrm{~b} / \mathrm{g} / \mathrm{n}$

- Soporte SSL/TLS

- WPA Enterprise security

- Motor de encriptación AES

- Conector UFL para antena externa

- Rango WiFi hasta $1 \mathrm{Km}$

Conectividad Sigfox

- Compatible zona RCZ1, Europa $868 \mathrm{MHz}$

- Rango de hasta $50 \mathrm{Km}$

- Dispositivo clase 0 . Máxima potencia de salida $+14 \mathrm{dBm}$

- Certificado por Sigfox

- Transceptor de banda estrecha TI CC1125

El diagrama de bloques del dispositivo desarrollado puede consultarse en la Figura 3.15.

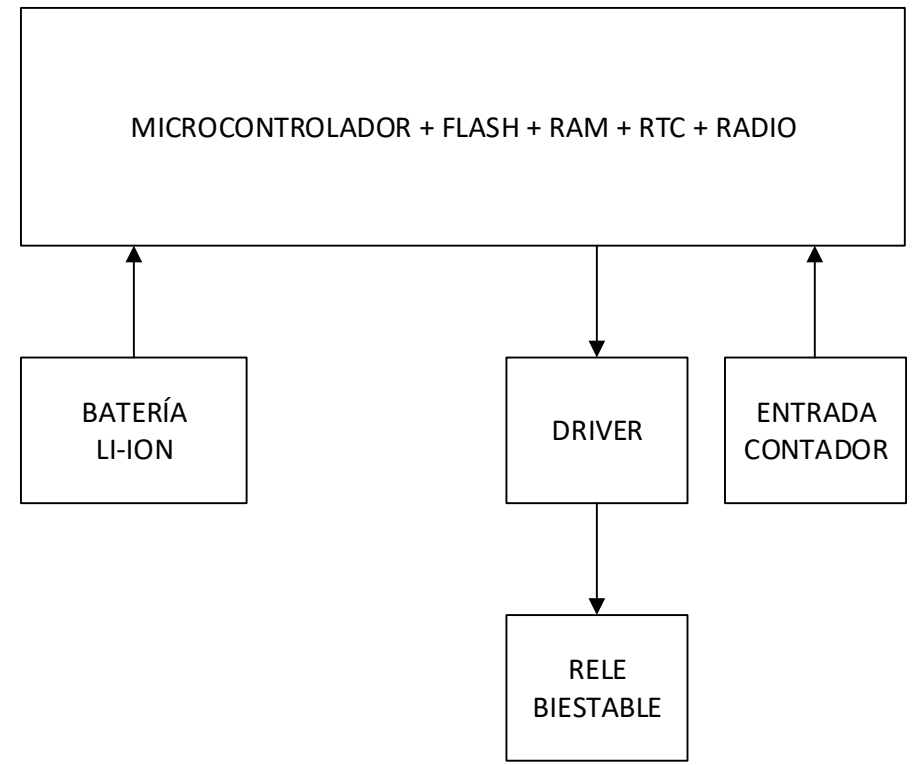

Figura 3.15. Diagrama de bloques. 
El esquemático electrónico se encuentra representado en la Figura 3.16.

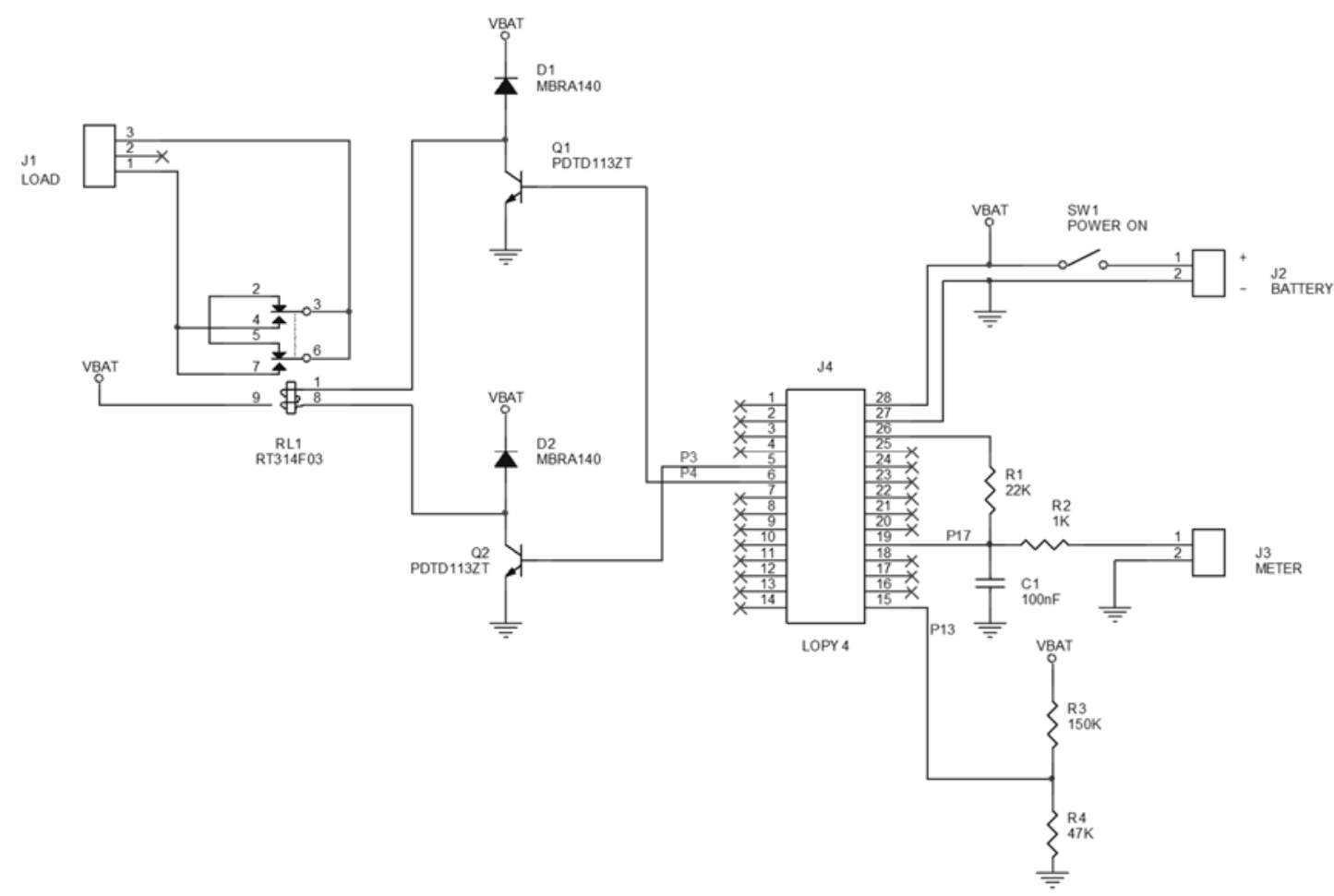

Figura 3.16. Esquemático electrónico.

Las señales del módulo de control utilizadas son las siguientes indicadas en la Tabla 3.2.

\begin{tabular}{|l|l|l|}
\hline Pin & Denominación & Función \\
\hline 28 & VIN & Batería de Litio 3.7 V \\
\hline 27 & GND & Masa \\
\hline 19 & P17 & Entrada de contador (Activa a nivel bajo) \\
\hline 05 & P3 & Salida de relé. Circuito cerrado. Carga activa \\
\hline 06 & P4 & Salida de relé. Circuito abierto. Carga no activa \\
\hline
\end{tabular}

Tabla 3.2. Señales en uso en el módulo de control.

Los cálculos eléctricos son los siguientes:

$$
\left.\begin{array}{r}
\mathrm{R}_{\text {bob typ }}=15 \Omega \rightarrow \mathrm{I}_{\mathrm{c} \text { typ }}=\frac{\mathrm{V}_{\mathrm{c}}}{\mathrm{R}_{\text {bob typ }}}=\frac{3}{15}=0.2 \mathrm{~A} \\
\mathrm{R}_{\text {bob max }}=16.5 \Omega \rightarrow \mathrm{I}_{\mathrm{c} \min }=\frac{\mathrm{V}_{\mathrm{c} \min }}{\mathrm{R}_{\text {bob max }}}=\frac{3}{16.5}=0.182 \mathrm{~A} \\
\mathrm{R}_{\text {bob min }}=13.5 \Omega \rightarrow \mathrm{I}_{\mathrm{c} \max }=\frac{\mathrm{V}_{\mathrm{c} \max }}{\mathrm{R}_{\text {bob min }}}=\frac{3.3}{13.5}=0.244 \mathrm{~A}
\end{array}\right\}
$$




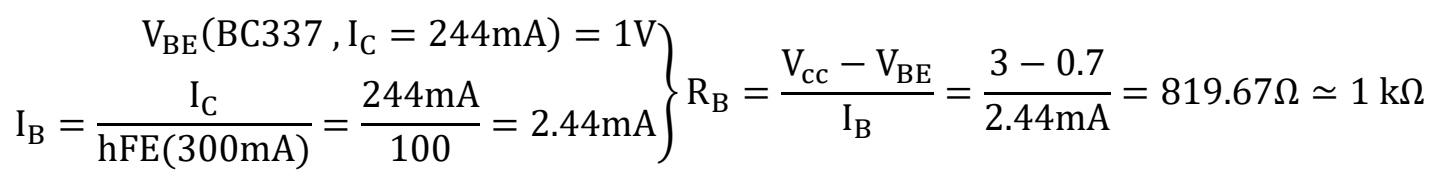

$$
\begin{aligned}
& \mathrm{R}_{\mathrm{B}-\mathrm{GND}}=10 \cdot \mathrm{R}_{\mathrm{B}}=10 \mathrm{k} \Omega
\end{aligned}
$$

Los conectores externos disponibles con su función y las características de la señal que manejan se encuentran listados en la Tabla 3.3.

\begin{tabular}{|l|l|l|}
\hline Contador & Tipo & $\begin{array}{l}\text { Industrial roscado de } 16 \mathrm{~mm} \text { con } 2 \text { pines } \\
\text { macho }\end{array}$ \\
\cline { 2 - 3 } & Función & $\begin{array}{l}\text { Entrada de señal procedente del contador sin } \\
\text { polarización }\end{array}$ \\
\cline { 2 - 3 } & Rango tensión & $3.3 \mathrm{~V}$ \\
\cline { 2 - 3 } & Corriente & $1 \mathrm{~mA}$ \\
\hline
\end{tabular}

\begin{tabular}{|l|l|l|}
\hline Salida & Tipo & $\begin{array}{l}\text { Industrial roscado de } 16 \mathrm{~mm} \text { con } 2 \text { pines } \\
\text { hembra }\end{array}$ \\
\cline { 2 - 3 } & Función & $\begin{array}{l}\text { Contacto libre de potencial para control de } \\
\text { electroválvula }\end{array}$ \\
\cline { 2 - 3 } & Rango tensión & $250 \mathrm{Vac}$ \\
\cline { 2 - 3 } & $\begin{array}{l}\text { Corriente } \\
\text { carga }\end{array}$ & $10 \mathrm{~A}$ \\
\hline
\end{tabular}

Tabla 3.3. Conectores y función.

Para una rápida verificación de cumplimiento de la funcionalidad requerida, se han conectado los componentes discretos necesarios para esta aplicación en una placa de circuito impreso pretaladrada para prototipado como puede verse en la Figura 3.17 junto también a la ya versión definitiva una vez terminado su montaje.
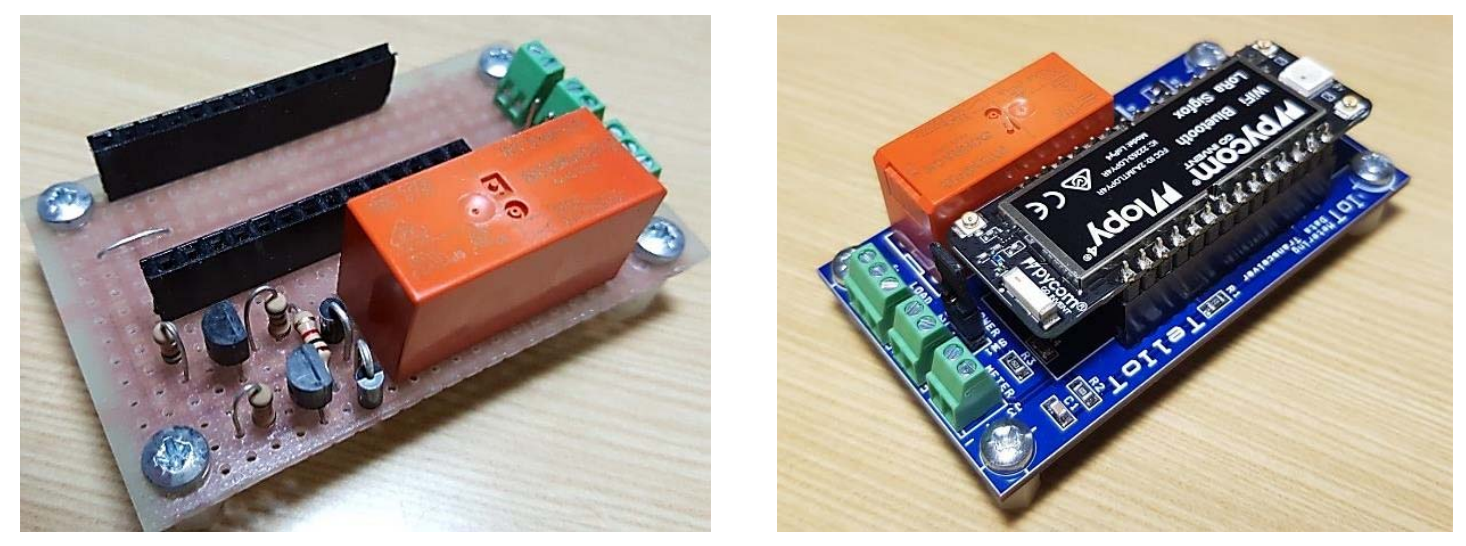

Figura 3.17. Placa de circuito.

Se ha diseñado y fabricado una placa de circuito impreso en la que se han utilizado componentes de montaje superficial tal y como puede verse en la Figura 3.18. 

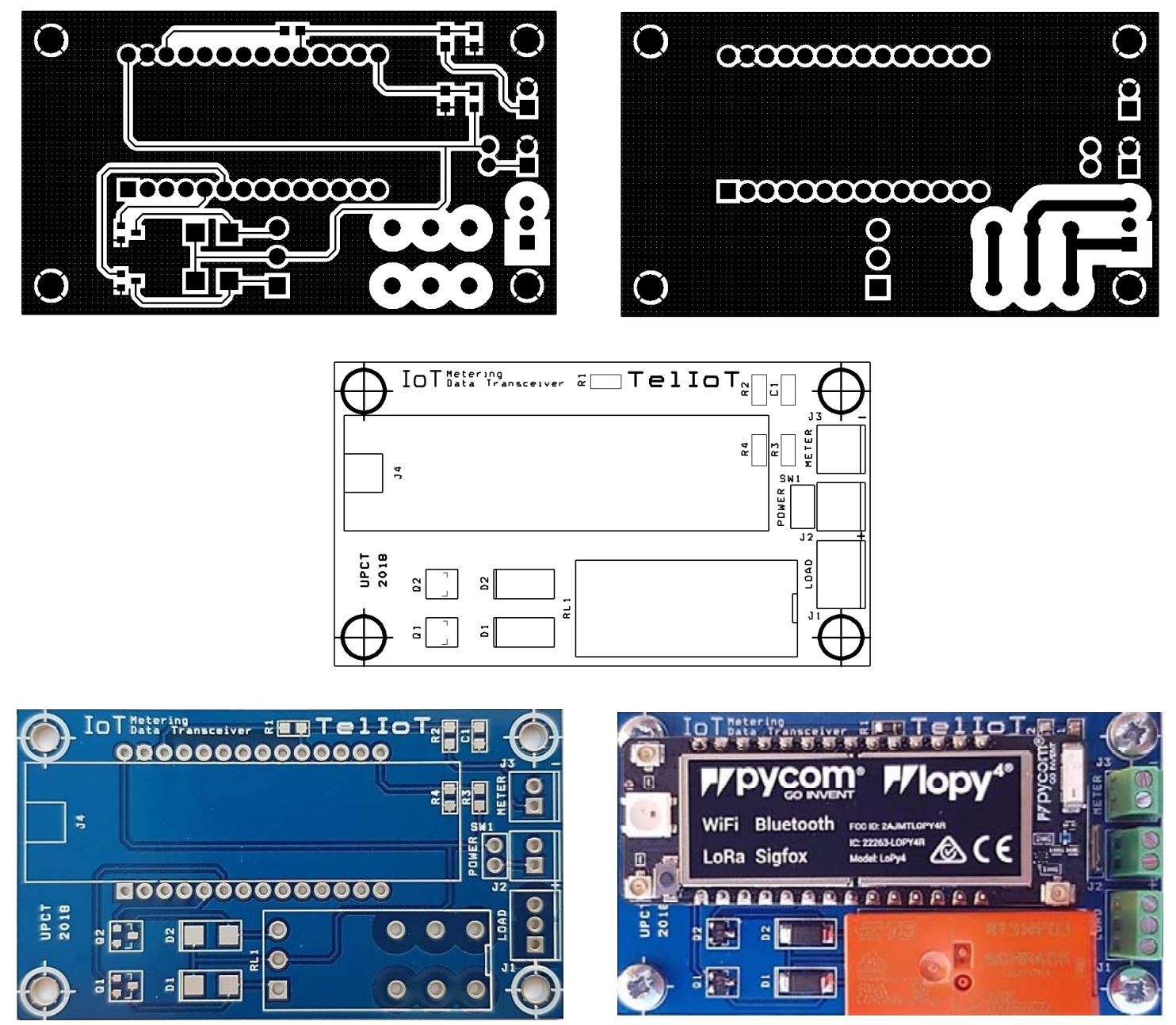

Figura 3.18. Circuito impreso con montaje SMD.

El cableado hacia los conectores externos de la carcasa y otros elementos en su interior, sigue el conexionado indicado en la Figura 3.19.

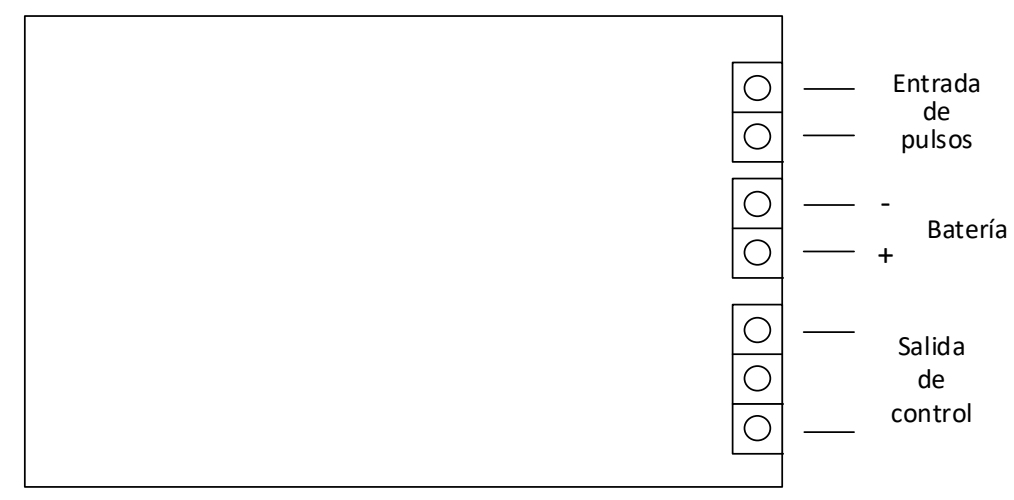

Figura 3.19. Conectores en placa.

El conexionado de la señal de consumo proveniente del contador sigue el diagrama de la Figura 3.20 . 


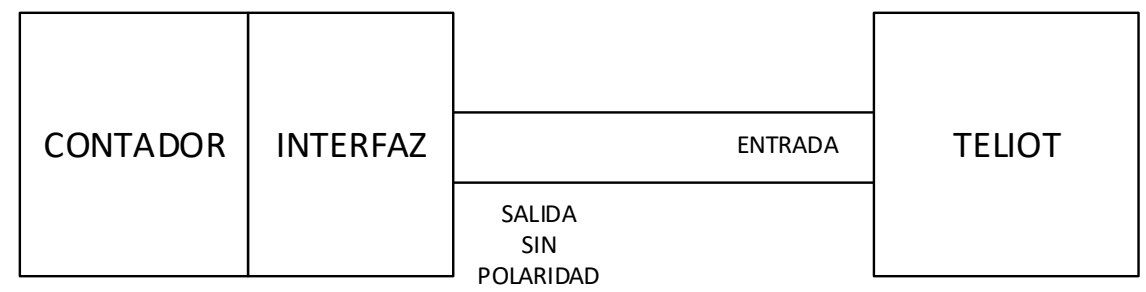

Figura 3.20. Diagrama de conjunto.

El conexionado del circuito de salida sigue el diagrama de la Figura 3.21.

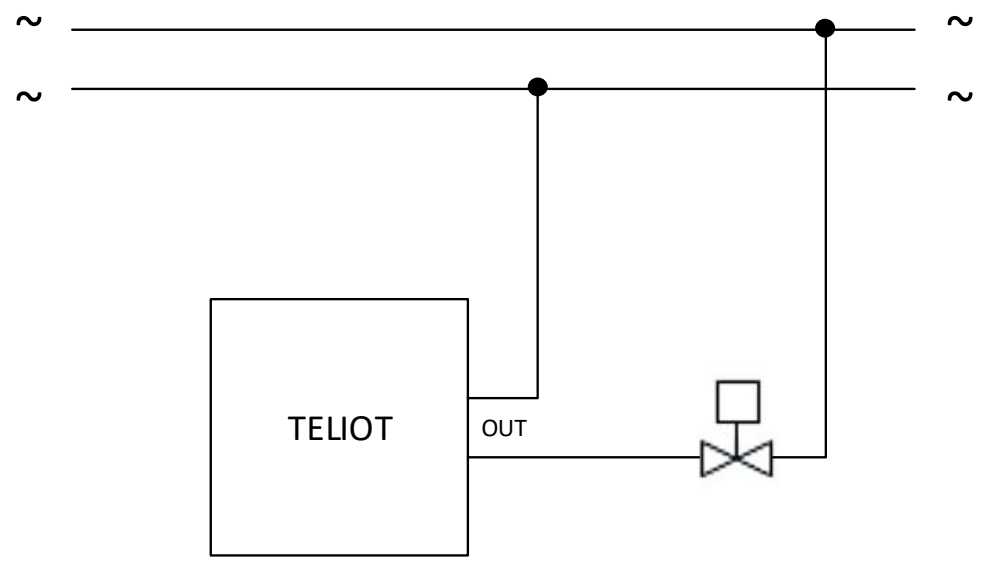

Figura 3.21. Conexionado del circuito de salida.

Aunque la batería incluida es de tipo secundaria y por tanto recargable, dada su capacidad y el bajo consumo a intervalos del dispositivo, con una sola carga la autonomía del dispositivo es aceptable para esta aplicación y por tanto no se ha previsto ningún elemento adicional para la recarga de la misma. Con esto se simplifica el dispositivo y se reducen costes. A pesar de ello es posible la recarga sin extraer la batería. Sólo es necesario desconectarla de la placa electrónica mediante la regleta atornillada y conectar su cableado a un cargador externo para baterías de lón-Litio. Las características del cargador han de ser las siguientes:

- Posibilidad de cargar baterías lón-Litio de $3.7 \mathrm{~V} / 2300 \mathrm{~mA}$

- Corriente recomendada de $1 \mathrm{~A}$

- Conector de salida capaz de aceptar cableado libre

No se debe cargar la batería estando conectada simultáneamente a esta unidad. Al finalizar la carga, se desconectará del cargador y volverá a conectarse a la placa del dispositivo respetando la polaridad indicada en la regleta: cable rojo a terminal $(+)$ y cable negro al terminal (-). La duración de la carga dependerá de la capacidad de suministro del cargador. Con un $1 \mathrm{~A}$ de salida, la carga puede durar 3.5 horas. Un ejemplo de cargador recomendado es la placa de evaluación MCP73871 de Microchip. Incluye el circuito integrado controlador de carga MCP73871 y la circuitería adicional necesaria para efectuar y monitorizar la recarga de baterías de lón-Litio tanto desde USB como desde un alimentador externo con diferentes opciones de carga seleccionables. Sus características son las siguientes: 
- Control de la corriente de entrada

- $A C-D C=1650 \mathrm{~mA}$

- USB High $=500 \mathrm{~mA}$

- USB Low = $100 \mathrm{~mA}$

- 3 LEDs indican estado de la carga, batería baja y alimentación ok

- Voltaje de carga prefijado a $4.2 \mathrm{~V}+-0.5 \%$ para baterías de lón-Litio

- Posible monitorización de temperatura mediante NTC

- Temporizador de seguridad de 6 horas

- Algoritmo de carga con voltaje constante y corriente constante

- Carga de baterías profundamente descargadas a $0.1 \mathrm{C}$

- Máxima corriente de carga programada a $1 \mathrm{~A}$

- Finalización de carga programada a $100 \mathrm{~mA}$ o automática

- Recarga automática

Para la conexión tanto de la batería como del alimentador, la placa dispone de terminales soldados que no disponen de ningún método de sujeción de los cables. Es recomendable preparar la placa para su uso soldando unos cables terminados en regleta para conectar la batería y un cable terminado en USB macho de tipo A para el alimentador. Es necesario además puentear con un cable los terminales CE (Charge Enable) y VDD para habilitar la carga. Las señales disponibles y su función se listan en la Tabla 3.4, la indicación de los LEDs en la Tabla 3.5 y una imagen de la placa ya preparada para su conexionado en la Figura 3.22.

\begin{tabular}{|c|c|c|}
\hline Terminal & Referencia & Descripción \\
\hline VDD & TP2 & Alimentación + \\
\hline VSS & TP1 & Alimentación - \\
\hline VBAT+ & TP4 & Batería + \\
\hline VSS & TP5 & Batería - \\
\hline CE & TP6 & Habilita carga a nivel alto \\
\hline
\end{tabular}

Tabla 3.4. Conexionado de la placa MCP73871.

\begin{tabular}{|l|c|c|c|}
\hline Indicación & $\begin{array}{c}\text { STAT1 } \\
\text { Verde }\end{array}$ & $\begin{array}{c}\text { STAT2 } \\
\text { Rojo }\end{array}$ & $\begin{array}{c}\text { PG } \\
\text { Azul }\end{array}$ \\
\hline Apagado & - & - & - \\
\hline Reposo & - & - & ON \\
\hline Carga en progreso & ON & - & ON \\
\hline Carga completa & - & ON & ON \\
\hline Fallo por temperatura & ON & ON & ON \\
\hline Fallo por temporización & ON & ON & ON \\
\hline Indicador de batería baja & ON & - & - \\
\hline Batería no presente & - & - & ON \\
\hline Sin alimentación de entrada & - & - & - \\
\hline
\end{tabular}

Tabla 3.5. Indicación de los LEDs de la placa MCP73871. 


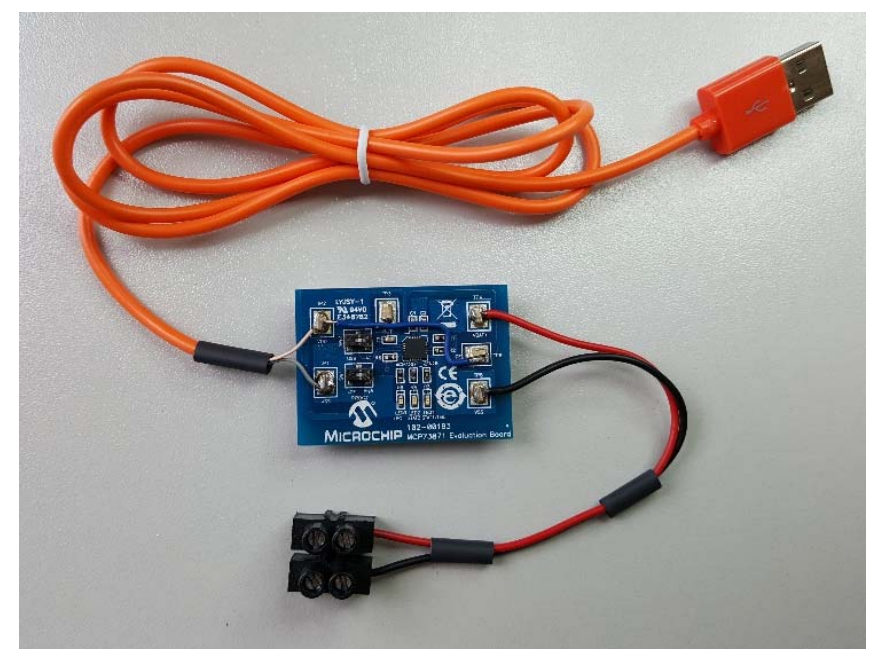

Figura 3.22. Imagen de la placa MCP73871 preparada para su uso.

\subsubsection{Desarrollo software}

El código del software embebido se encuentra escrito en MicroPhyton debido a que el módulo Pycom LoPy4 incluye un intérprete del mismo para las aplicaciones loT de usuario.

La operativa del software embebido en el microcontrolador del dispositivo es la siguiente. Se comienza comprobando la causa del inicio de la ejecución. Los posibles casos son por conexión de alimentación la primera vez, por cambio de estado en la entrada y por vencimiento del temporizador. Si se trata de la primera ejecución al conectar la alimentación, se procede a hacer una transmisión de datos con solitud de respuesta para obtener la configuración, se fija el intervalo entre futuras transmisiones y se pasa al modo de bajo consumo.

Si se ha salido del modo de bajo consumo por cambio de estado en el pin digital de entrada al recibir la señal de pulsos del contador, se lee el valor actual del contador de la memoria no volátil, se actualiza y se guarda. Seguidamente se vuelve al modo de bajo consumo. Para permitir esta operación se ha incluido una resistencia de pull-up externa en el pin digital al que está conectada la entrada de contador, y se habilita por software el despertar por cambio a nivel bajo de esta señal de entrada.

En el caso de haberse cumplido el temporizador que despierta al dispositivo periódicamente, se comprueba si han transcurrido el intervalo entre transmisiones configurado. En ese caso, se procede a comprobar si corresponde una transmisión con solicitud de bajada, algo que se efectúa una vez al día. En caso contrario se lleva a cabo una comunicación sólo de subida. Los contadores de minutos transcurridos entre transmisiones y entre transmisiones con bajada, son inicializados si corresponde. Si se recibe de la red una trama de bajada, se procesa la información en ella contenida. Esto incluye el intervalo de tiempo entre transmisiones y el estado de la salida.

Al pasar al modo de bajo consumo se habilita el despertar transcurridos 20 segundos, que es el máximo que se ha comprobado que se puede utilizar con este módulo antes de que el temporizador watchdog actúe y resetee el módulo.

Un diagrama de intervalos de actividad puede consultarse en la Figura 3.23. 


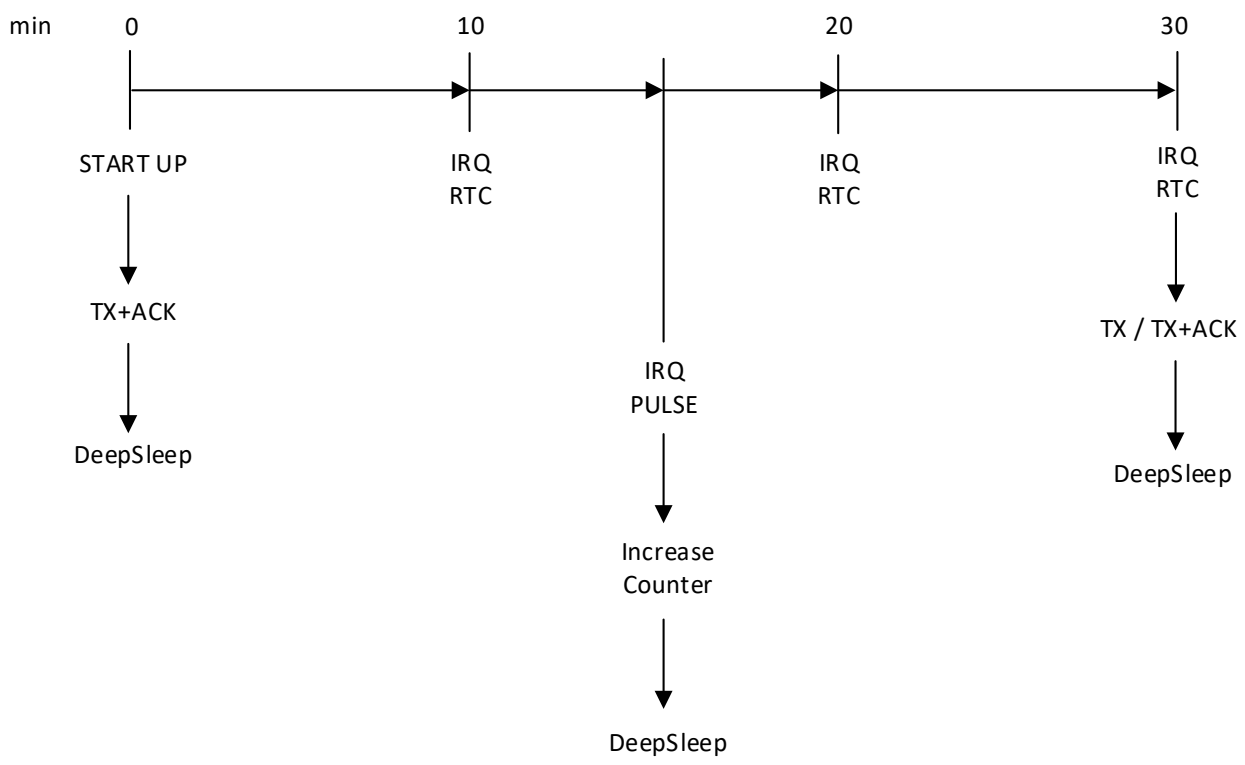

Figura 3.23. Diagrama de intervalos de actividad.

El diagrama de flujo del software embebido puede consultarse en la Figura 3.24.
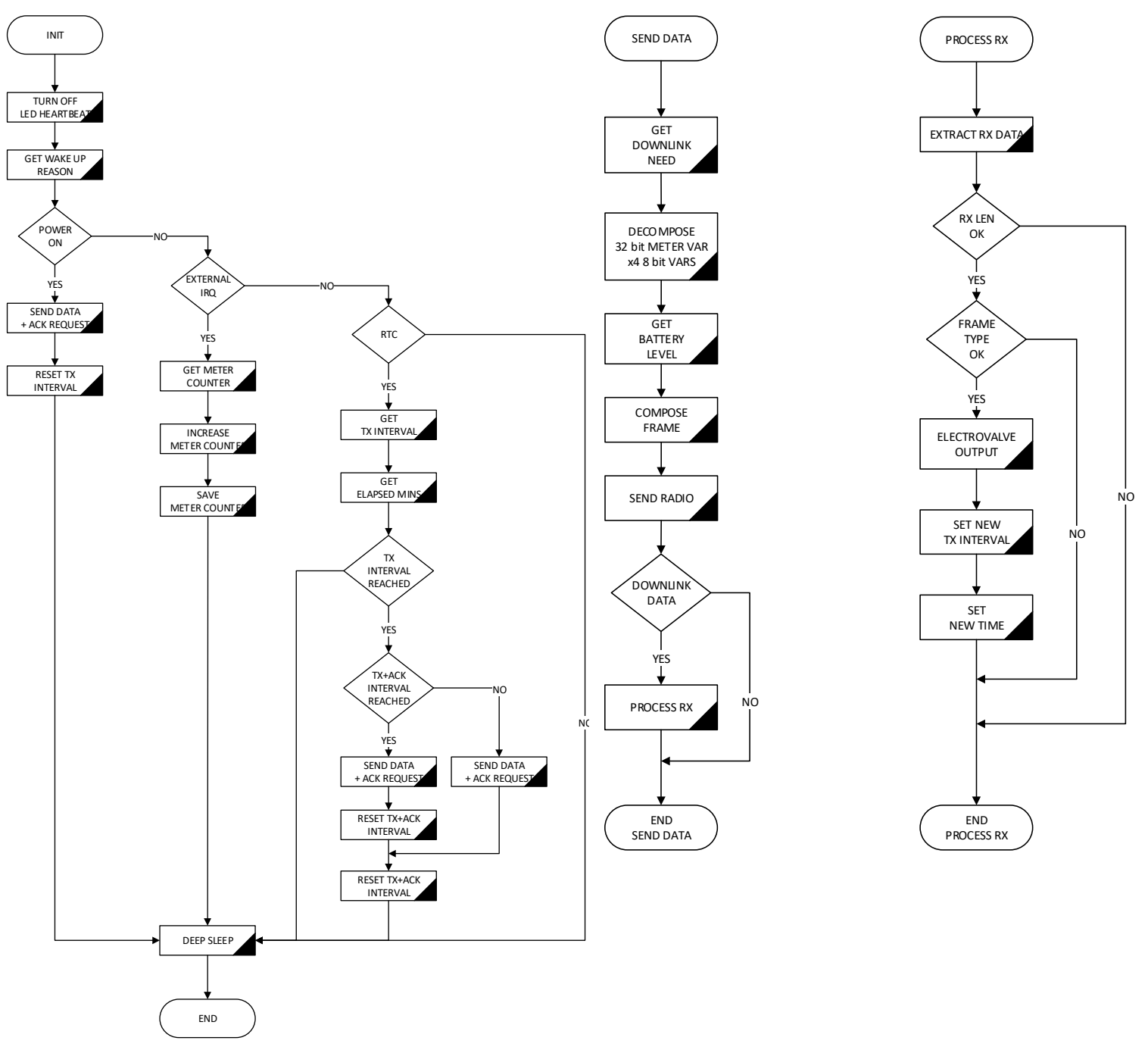

Figura 3.24. Flujograma del firmware del dispositivo PyCom. 
Un diagrama de funciones por nivel del software está representado en la Figura 3.25.

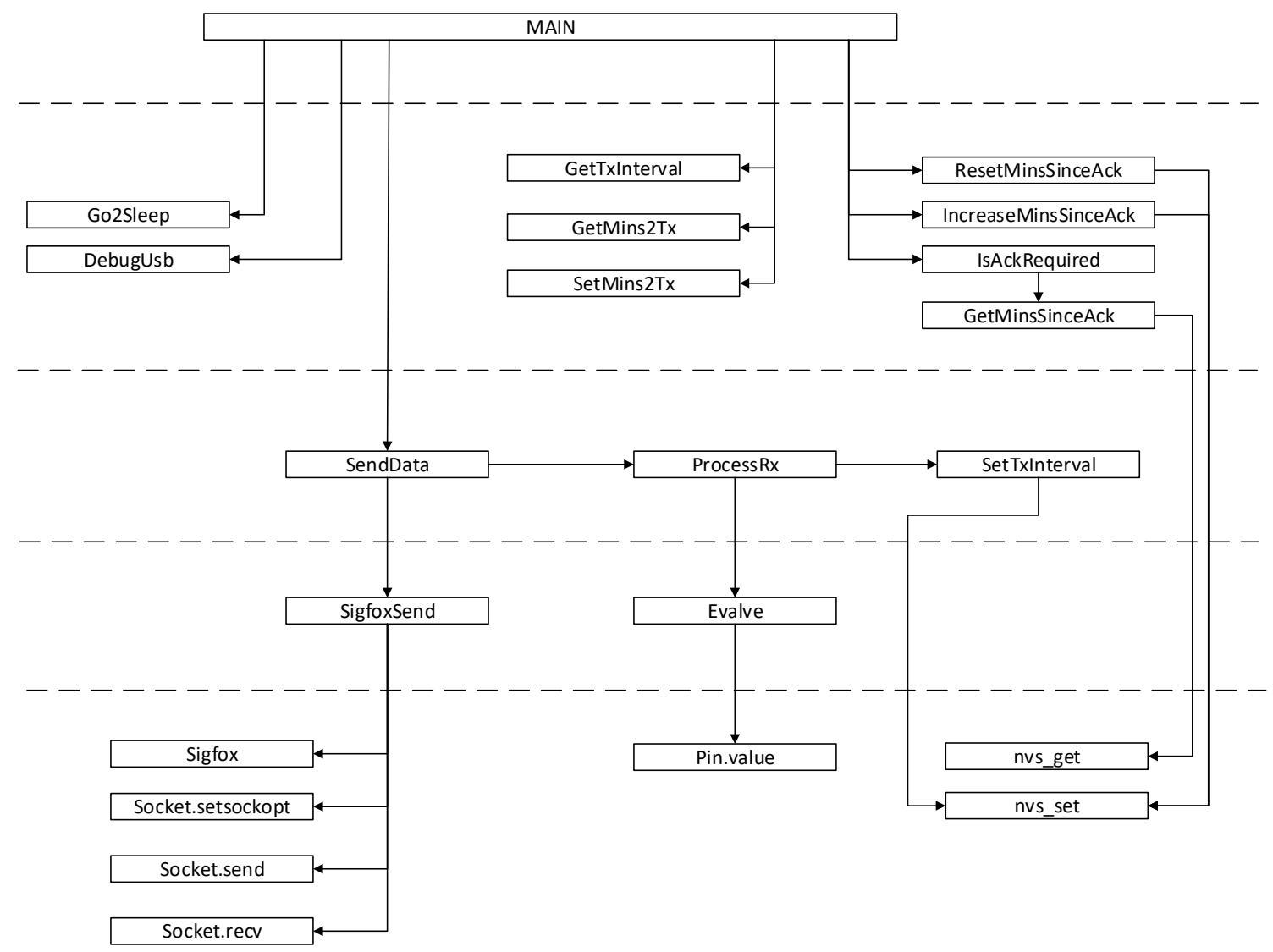

Figura 3.25. Diagrama de funciones por nivel del dispositivo PyCom.

La trama enviada por el dispositivo hacia la red Sigfox sigue el formato mostrado en la Figura 3.26 .

\begin{tabular}{|c|c|c|c|c|c|c|c|c|}
\hline BYTE & 00 & 01 & 02 & 03 & 04 & 05 & 06 & 07 \\
\hline INFO & $\begin{array}{l}\text { FRAME } \\
\text { TYPE }\end{array}$ & \multicolumn{4}{|c|}{ METER VALUE } & DEVICE & STATUS & BATTERY \\
\hline $\begin{array}{c}\text { EXAMPLE } \\
\text { (HEX) }\end{array}$ & 01 & & $\odot \odot \odot$ & $01 \mathrm{D}$ & & 05 & $\odot \odot$ & 58 \\
\hline
\end{tabular}

Figura 3.26. Trama de subida de dispositivo a red.

La longitud de la trama es de 8 bytes (64 bits) y la descripción de los campos puede consultarse en la Tabla 3.6.

\begin{tabular}{|l|ll|}
\hline FRAME TYPE & DESCRIPTION: & Frame type code \\
\cline { 2 - 3 } & LENGHT: & 1 byte / 8 bits \\
\cline { 2 - 3 } & VALUES: & 1: Water meter data \\
\hline \multirow{3}{*}{ METER VALUE } & DESCRIPTION: & Accumulated meter value \\
\cline { 2 - 3 } & LENGHT: & 4 bytes / 32 bits \\
\cline { 2 - 3 } & VALUES: & $0-4294967295$ \\
\hline
\end{tabular}




\begin{tabular}{|l|ll|}
\hline \multirow{3}{*}{ DEVICE } & DESCRIPTION: & Device identifier \\
\cline { 2 - 3 } & LENGHT: & 1 byte / 8 bits \\
\cline { 2 - 3 } & VALUES: & 0: Direct link \\
& & 1-255: Device-id via gateway \\
\hline
\end{tabular}

\begin{tabular}{|l|ll|}
\hline \multirow{3}{*}{ STATUS } & DESCRIPTION: & Operational status \\
\cline { 2 - 3 } & LENGHT: & 1 byte $/ 8$ bits \\
\cline { 2 - 3 } & VALUES: & $\begin{array}{l}0: \text { No error } \\
0-10: \text { Error code }\end{array}$ \\
\hline
\end{tabular}

\begin{tabular}{|l|ll|}
\hline \multirow{3}{*}{ BATTERY } & DESCRIPTION: & Battery power level \\
\cline { 2 - 3 } & LENGHT: & 1 byte $/ 8$ bits \\
\cline { 2 - 3 } & VALUES: & $0-100:$ Battery $\%$ power level \\
\hline
\end{tabular}

Tabla 3.6. Descripción de los campos de la trama de subida.

La trama recibida desde la red Sigfox es acorde al siguiente formato de la Figura 3.27.

\begin{tabular}{|c|c|c|c|c|c|c|c|c|}
\hline BYTE & 00 & 01 & 02 & 03 & 04 & 05 & 06 & 07 \\
\hline INF0 & $\begin{array}{c}\text { FRAME } \\
\text { TYPE }\end{array}$ & OUTPUT & $\begin{array}{c}\text { INTERVAL } \\
\text { UNITS }\end{array}$ & $\begin{array}{c}\text { TX } \\
\text { INTERVAL }\end{array}$ & \multicolumn{2}{|c|}{ TIME } \\
\hline $\begin{array}{c}\text { EXAMPLE } \\
\text { (HEX) }\end{array}$ & 01 & 00 & 01 & 07 & & 5 A $78 \quad 2 C \quad 37$ & \\
\hline
\end{tabular}

Figura 3.27. Trama de bajada de red a dispositivo.

La longitud de la trama de bajada es de 8 bytes (64 bits) y la descripción de los campos es presente en la Tabla 3.7.

\begin{tabular}{|c|c|c|}
\hline \multirow{3}{*}{$\begin{array}{l}\text { FRAME } \\
\text { TYPE }\end{array}$} & DESCRIPTION: & Frame type code \\
\hline & LENGHT: & 1 byte / 8 bits \\
\hline & VALUES: & 1: Water meter configuration \\
\hline \multirow[t]{3}{*}{ OUTPUT } & DESCRIPTION: & New output value \\
\hline & LENGHT: & 1 bytes / 8 bits \\
\hline & VALUES: & $0-255$ \\
\hline \multirow{3}{*}{$\begin{array}{l}\text { INTERVAL } \\
\text { UNITS }\end{array}$} & DESCRIPTION: & Delivery interval units \\
\hline & LENGHT: & 1 byte / 8 bits \\
\hline & VALUES: & $\begin{array}{l}\text { 01: Minutes } \\
\text { 02: Hours } \\
\text { 03: Days }\end{array}$ \\
\hline \multirow{3}{*}{$\begin{array}{c}\text { TX } \\
\text { INTERVAL }\end{array}$} & DESCRIPTION: & Delivery interval \\
\hline & LENGHT: & 1 byte / 8 bits \\
\hline & VALUES: & $0-255$ \\
\hline \multirow[t]{3}{*}{ TIME } & DESCRIPTION: & $\begin{array}{l}\text { Current date and time in EPOC } \\
\text { format (seconds from 1970) }\end{array}$ \\
\hline & LENGHT: & 4 bytes / 32 bits \\
\hline & VALUES: & $0-4294967295$ \\
\hline
\end{tabular}

Tabla 3.7. Descripción de los campos de la trama de bajada. 
Dado que el dispositivo obtiene su energía de una batería interna y con objeto de maximizar la duración de esta, en ausencia de actividad se pasa a un modo de ahorro de energía con un consumo mínimo. Las interrupciones externa y del temporizador sacan al procesador de este modo y se reanuda la operación llevando a cabo las tareas correspondientes antes de volver a modo de bajo consumo.

Para retener información aunque la batería se agotase o desconectase y el dispositivo quedase sin energía, se almacena el valor de determinadas variables en la memoria no volátil de tipo EEPROM integrada en el módulo de control. Las variables retenidas, su uso y las funciones que las utilizan se listan en la Tabla 3.8.

\begin{tabular}{|c|l|l|}
\hline \multicolumn{1}{|c|}{ VARIABLE } & \multicolumn{1}{|c|}{ DESCRIPCIÓN } & \multicolumn{1}{c|}{ FUNCIONES } \\
\hline TxInterval_Mins & Periodo de minutos entre envíos. & $\begin{array}{l}\text { GetTxInterval() } \\
\text { SetTxInterval() }\end{array}$ \\
\hline TxInterval_Mins & Periodo de minutos entre envíos. & $\begin{array}{l}\text { GetTxInterval() } \\
\text { SetTxInterval() }\end{array}$ \\
\hline Elapsed_Mins & Minutos desde el último envío. & $\begin{array}{l}\text { GetMinsTx() } \\
\text { SetMinsTx() }\end{array}$ \\
\hline AckDay & Número del día que se solicitó el ack. & $\begin{array}{l}\text { GetLastAckDay() } \\
\text { SetLastAckDay() }\end{array}$ \\
\hline Mins_Since_Ack & Número de mins desde el último ack. & $\begin{array}{l}\text { IsAckRequired() } \\
\text { GetMinsSinceAck () } \\
\text { ResetMinsSinceAck () } \\
\text { IncreaseMinsSinceAck() }\end{array}$ \\
\hline
\end{tabular}

Tabla 3.8. Variables en memoria no volátil.

\subsubsection{Gateway loT LoRa-Sigfox}

\subsubsection{Descripción}

El dispositivo a continuación descrito tiene como finalidad el facilitar la obtención de información procedente de contadores de consumo de agua situados en ubicaciones donde no es posible enlazar directamente con la red Sigfox por no estar disponible su cobertura en dicha localización.

Para realizar esta labor, el presente dispositivo se sitúa en una ubicación intermedia donde esté presente la cobertura de la red Sigfox y donde a su vez sea posible establecer enlace radio directo con el dispositivo de lectura de contador. Este enlace punto a punto se lleva a cabo utilizando tecnología radio LoRa, la cual está diseñada también para dar soporte a objetos conectados en el llamado Internet de las cosas y permite maximizar tanto el alcance de la señal radio como la autonomía del dispositivo. Ambos enlaces radio, LoRa y Sigfox, se llevan a cabo en bandas ISM cumpliendo los requerimientos de estas, con lo que no es necesario una licencia de operación por parte del organismo regulador del espectro radioeléctrico.

La operativa del dispositivo consiste en mantener recepción permanente en banda ISM a la espera de una transmisión de datos procedente del dispositivo de telemedida y telecontrol ubicado junto al contador del abonado. Un mismo gateway puede enlazar con hasta 255 
dispositivos de contador siempre que dispongan de radio LoRa en la misma banda ISM que el gateway y sean compatibles con el protocolo más adelante descrito. La información de consumo obtenida de los contadores, es almacenada en una memoria no volátil y es enviada posteriormente a la red Sigfox a los intervalos establecidos, ya que hay limitaciones tanto de ocupación del canal radio al operar en banda ISM como del máximo número de tramas que se pueden enviar a la red según el contrato con el operador. El dispositivo puede verse en la Figura 3.28 .
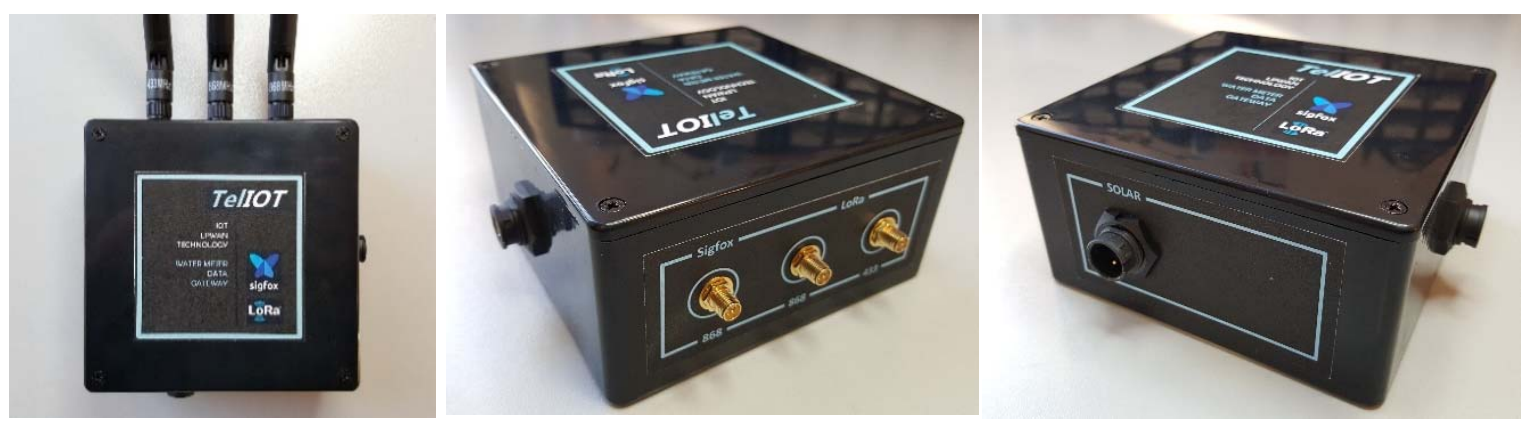

Figura 3.28. Vista exterior del dispositivo gateway.

\subsubsection{Desarrollo hardware}

El equipo consta de una placa base donde se ubican el microcontrolador, la memoria no volátil, el reloj en tiempo real RTC, regulador de tensión y controlador de carga de la batería. Esta placa principal sirve de soporte físico e interconexión eléctrica con dos módulos de comunicaciones, el primero de ellos compatible con LoRa / LoRaWAN y el segundo con Sigfox.

Los módulos de comunicaciones disponen de un conector de radiofrecuencia para antena. Esta señal se ha llevado mediante un latiguillo de cable al borde de la caja para poder situar las antenas en el exterior y hacer más fácil su intercambio. Hay posibilidad de acoplar tres antenas simultáneamente, una para Sigfox en $868 \mathrm{MHz}$ y dos para LoRaWAN tanto en 868 como en 434 $\mathrm{MHz}$.

La conexión USB de la placa principal también se ha dispuesto en la carcasa usando un conector de panel de tipo Micro-USB. Mediante este, puede alimentarse el dispositivo externamente, recargar la batería interna o realizar actualizaciones de firmware.

La batería de tecnología lón-Litio incorporada, permite mantener el funcionamiento en casos de falta de suministro eléctrico externo o llevar a cabo una operación autónoma mediante su recarga con un panel solar externo. Un conector industrial roscado de dos pines, situado en la parte inferior, permite la conexión de este. En la Figura 3.29 se encuentra una vista interior del dispositivo y de operación con panel solar externo. 

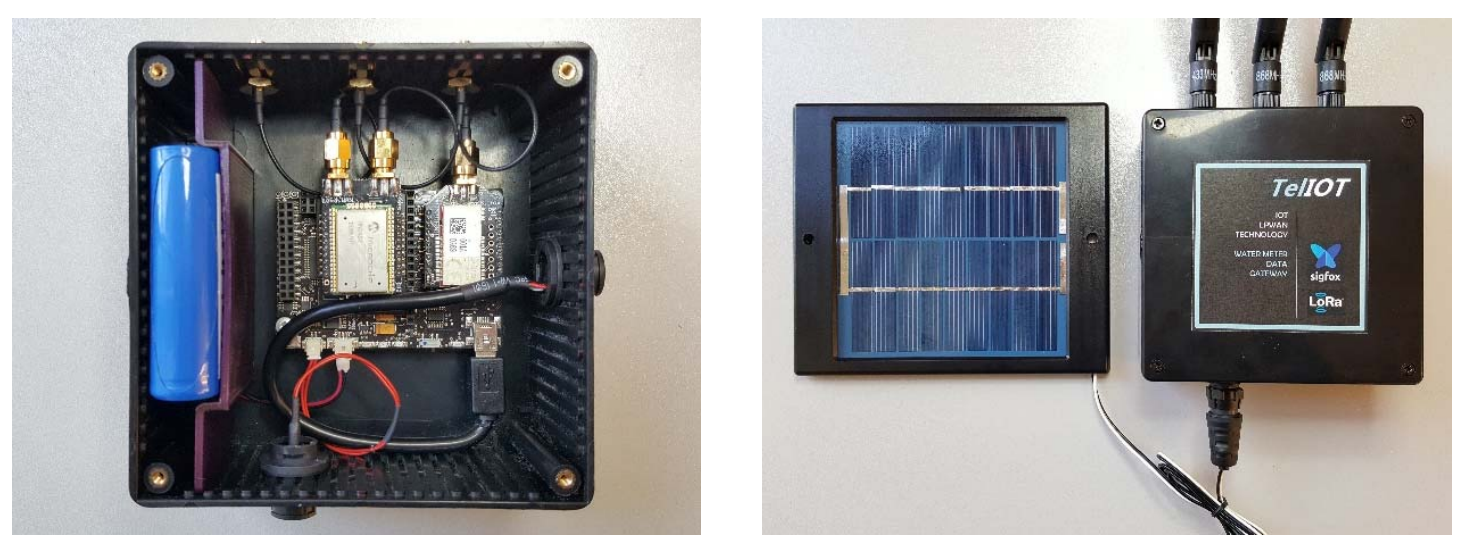

Figura 3.29. Vista interior del dispositivo gateway y conexión de panel solar.

El conjunto se encuentra protegido mediante una carcasa de material ABS en cuyos laterales se sitúan los diferentes conectores para las antenas, el suministro eléctrico y para posibles actualizaciones del software embebido. Un diagrama de bloques del dispositivo puede verse en la Figura 3.30.

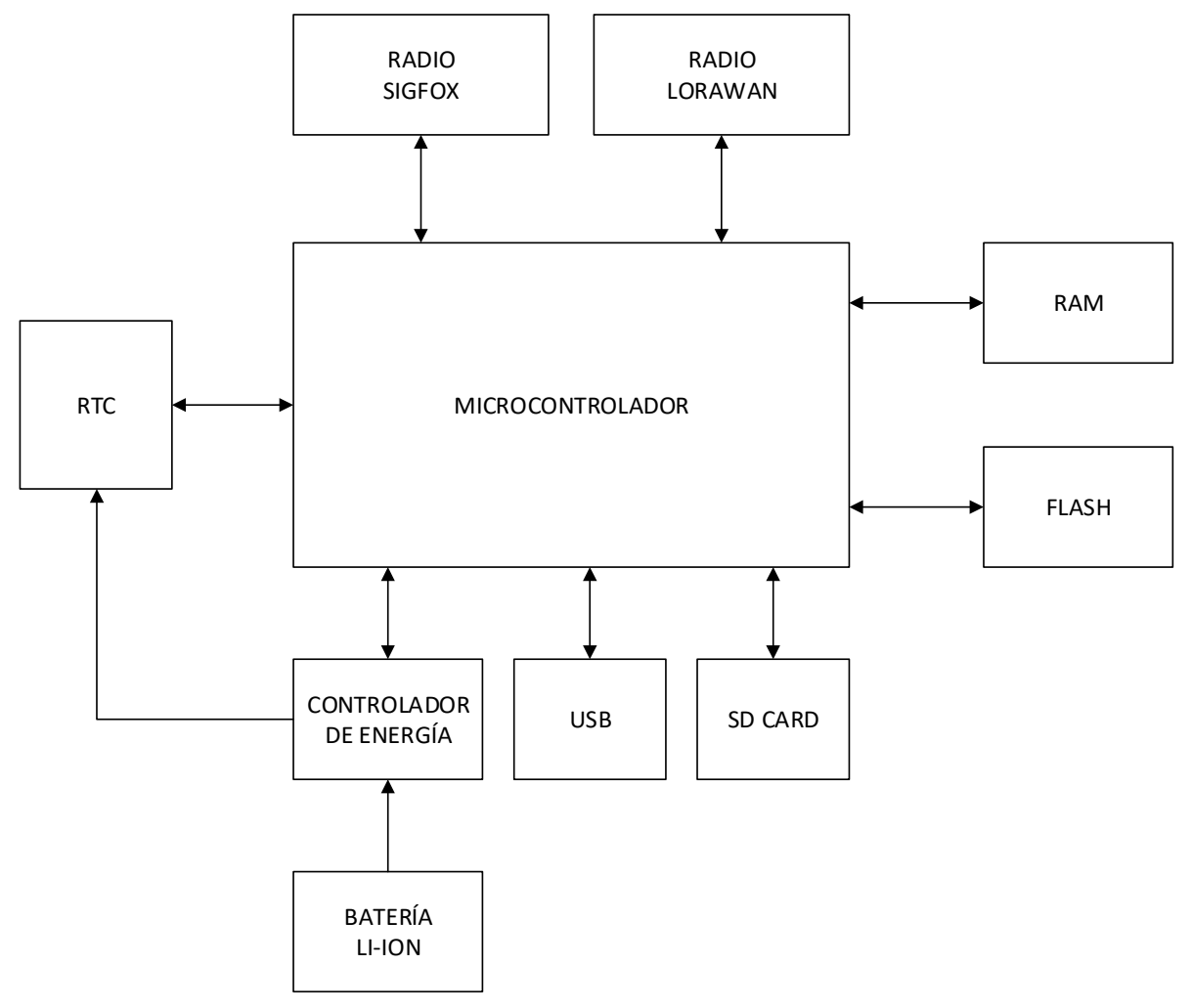

Figura 3.30. Diagrama de bloques del dispositivo gateway. 
El esquemático eléctrico se encuentra representado a continuación en la Figura 3.31.
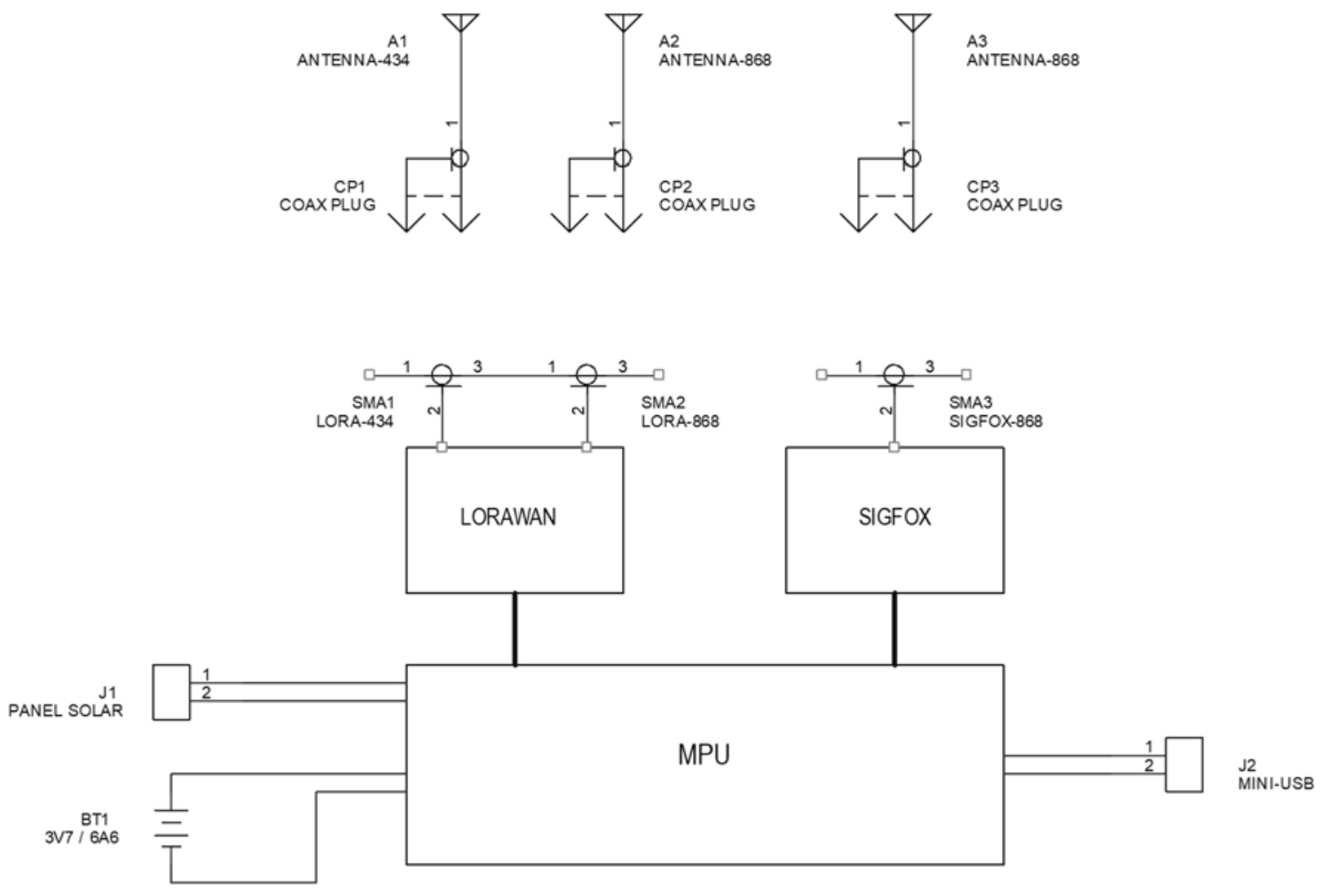

Figura 3.31. Esquemático eléctrico del dispositivo gateway.

Los conectores externos disponibles con su función y características de la señal que manejan se encuentran listados en la Tabla 3.9.

\begin{tabular}{|c|c|c|}
\hline \multirow[t]{5}{*}{ USB } & Tipo & Micro-USB tipo A hembra \\
\hline & Función & Actualizaciónes firmware / Carga de batería \\
\hline & Tensión & $5 \mathrm{~V}$ \\
\hline & Corriente carga & $100 \mathrm{~mA}$ \\
\hline & Tiempo carga & 66 h $(0-100 \%)$ \\
\hline \multirow[t]{5}{*}{ Panel solar } & Tipo & $\begin{array}{l}\text { Industrial roscado de } 16 \mathrm{~mm} \text { con } 2 \text { pines } \\
\text { macho }\end{array}$ \\
\hline & Función & $\begin{array}{l}\text { Entrada de tensión para recarga de batería } \\
\text { mediante panel solar externo }\end{array}$ \\
\hline & Rango tensión & $6-12 \mathrm{~V}$ \\
\hline & Corriente carga & $280 \mathrm{~mA}$ \\
\hline & Tiempo carga & $24 \mathrm{~h}(0-100 \%)$ \\
\hline \multirow{3}{*}{$\begin{array}{l}\text { Antena Sigfox } \\
868\end{array}$} & Tipo & SMA hembra invertido \\
\hline & Frecuencia & $868 \mathrm{MHz}$ \\
\hline & Potencia & $14 \mathrm{dBm}$ \\
\hline \multirow{3}{*}{$\begin{array}{l}\text { Antena Lora } \\
868\end{array}$} & Tipo & SMA hembra invertido \\
\hline & Frecuencia & $868 \mathrm{MHz}$ \\
\hline & Potencia & $14 \mathrm{dBm}$ \\
\hline
\end{tabular}




\begin{tabular}{|c|c|c|}
\hline \multirow{3}{*}{$\begin{array}{ll}\text { Antena } & \text { Lora } \\
433 & \end{array}$} & Tipo & SMA hembra invertido \\
\hline & Frecuencia & $433 \mathrm{MHz}$ \\
\hline & Potencia & $14 \mathrm{dBm}$ \\
\hline
\end{tabular}

Tabla 3.9. Conectores externos del dispositivo gateway.

El proceso de carga de batería puede llevarse a cabo mediante alimentador externo y a través del conector USB o mediante un panel solar a través del conector específico. En el caso de alimentador externo, este ha de tener las siguientes características:

- Tensión constante de $5 \mathrm{~V}$.

- Corriente mínima de $500 \mathrm{~mA}$.

- Conector de salida macho tipo mini-USB.

Para iniciar la carga se ha de conectar el cargador al conector de tipo Mini-USB situado en el lateral del dispositivo. Si se retira la tapa y se visualiza el interior, se puede ver como se ilumina un LED rojo en la placa base cuando la batería está cargando y se apaga cuando la carga ha finalizado. En función del estado de descarga de la batería, el proceso de recarga puede durar hasta 66 horas. Para la recarga mediante panel solar, se utilizará uno con las siguientes características:

- Tensión entre 6 y $12 \mathrm{~V}$.

- Corriente mínima de $280 \mathrm{~mA}$.

- Conector circular de dos polos para panel respetando la polaridad.

Para iniciar la carga, la tensión procedente del panel solar se ha de hacer llegar por el conector situado en el lateral inferior del dispositivo respetando la polaridad como se muestra en la Figura 3.32. El panel solar puede dejarse permanentemente conectado y la batería se recargará cuando su nivel disminuya y el panel sea capaz de suministrar energía suficiente. Si se retira la tapa y se visualiza el interior, se puede ver un led rojo iluminado en la placa base cuando la batería está cargando. Este led se apaga cuando la carga finaliza. En función del estado de descarga de la batería, el proceso de recarga puede durar hasta 24 horas.

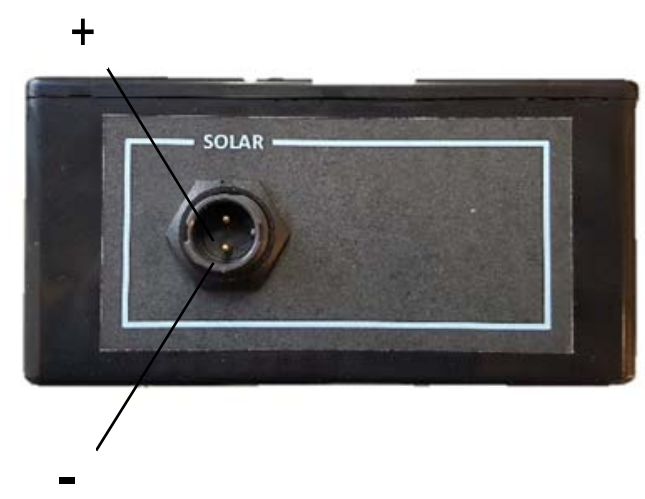

Figura 3.32. Polaridad del conector para panel solar del dispositivo gateway.

Los micro-interruptores de la placa base mostrados en la Figura 3.33 permiten el encendido y habilitar el modo de hibernación. Han de configurarse de la siguiente forma:

- Micro-interruptor superior izquierdo.

Función: POWER (Alimentación del dispositivo). 
Posición: ON / Hacia LEDs (izquierda mirando desde arriba).

- Micro-interruptor inferior izquierdo.

Función: HIBERNATE (Habilita el modo de hibernación).

Posición: OFF / Hacia LEDs (izquierda mirando desde arriba).

- Micro-interruptor superior derecho (versión de placa 1.5).

Función: WATCHDOG (Habilita el temporizador de guarda ante cuelgues).

Posición: OFF.
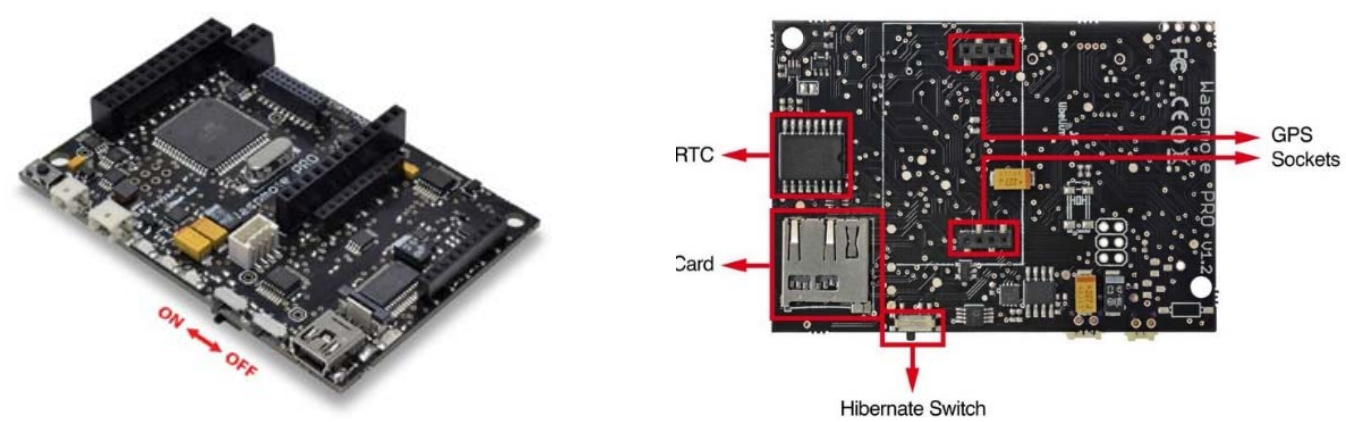

Figura 3.33. Micro-interruptores de configuración de placa Waspmote.

\subsubsection{Desarrollo software}

Se describe a continuación la operativa del software embebido en el dispositivo. Tras el encendido, se procede a inicializar los módulos radio Sigfox y LoRa. En el caso de LoRa, se establece el modo de funcionamiento para enlace directo sin capa LoRaWAN. Se configuran la banda radio, frecuencia, potencia, factor de ensanchamiento, velocidad, ancho de banda y verificación de redundancia cíclica.

Se pasa a la espera continua de una trama LoRa que sea válida acorde con el formato para dispositivos compatibles con el protocolo Teliot aquí descrito. Si esta se recibe, se procede a su envío a la red Sigfox a través de la radio correspondiente. Si se trata de una trama con requisito de respuesta, esta se solicita a su vez a la red Sigfox. En este caso, una vez recibida la trama de bajada y verificada, se extrae el paquete de información útil para Teliot y se reenvía a través del enlace LoRa hacia el dispositivo que inició la comunicación.

El anterior proceso se repite continuamente. Para garantizar la plena disponibilidad en recepción de tramas LoRa procedentes de los dispositivos de telemedida y telecontrol de contador, el software embebido de este gateway no detiene el procesador ni la radio LoRa. En el caso de la radio Sigfox, sí se lleva a cabo su encendido antes de enviar una trama y un apagado al finalizar. El flujograma del software embebido del dispositivo gateway puede consultarse en la Figura 3.34 . 

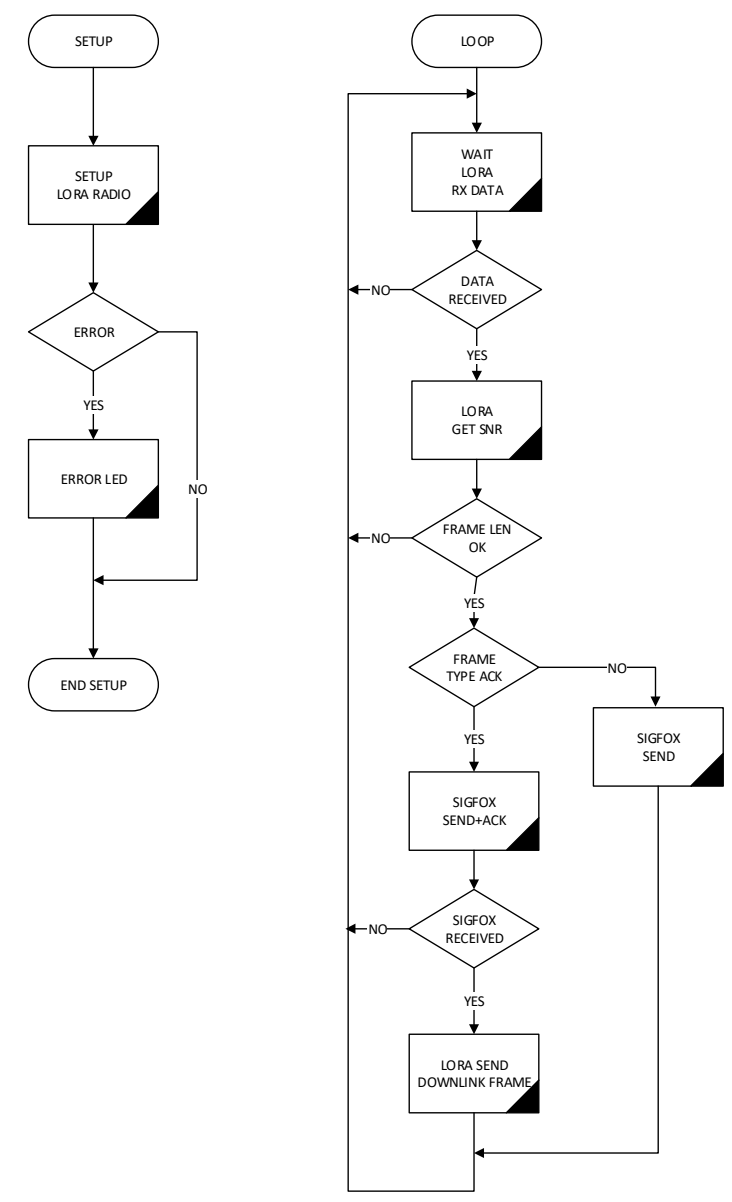

Figura 3.34. Flujograma del software embebido del dispositivo gateway.

La comunicación entre un dispositivo de contador y el gateway se efectúa acorde al siguiente protocolo mostrado en la Figura 3.35.

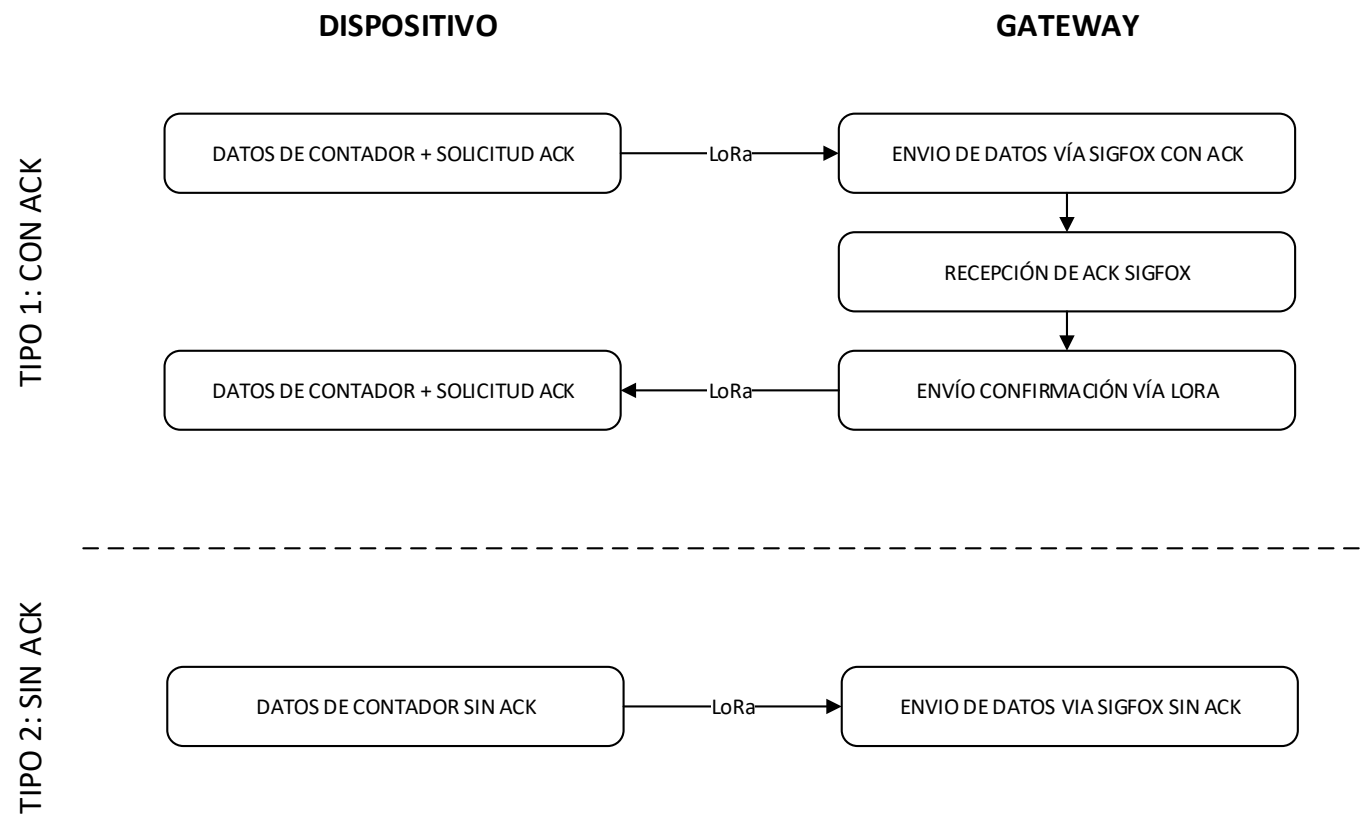

Figura 3.35. Diagrama de comunicación entre dispositivo de telemedida y gateway. 
El gateway con mayor disponibilidad energética permanece siempre a la escucha y puede recibir tramas en cualquier momento. Los dispositivos de contador se mantienen en modo de bajo consumo para prolongar su autonomía y sólo despiertan para enviar una trama a los intervalos prefijados. Esta comunicación puede ser unidireccional o de tipo 2, enviando el dato y pasando de nuevo a bajo consumo sin esperar confirmación, o de tipo 1, enviando el dato y permaneciendo a la espera por un periodo de tiempo para recibir una trama de confirmación y configuración.

La trama enviada por el dispositivo mediante LoRa ha de seguir el formato mostrado en la Figura 3.36.

\begin{tabular}{|c|c|c|c|c|c|c|c|c|}
\hline BYTE & 00 & 01 & 02 & 03 & 04 & 05 & 06 & 07 \\
\hline INFO & $\begin{array}{l}\text { FRAME } \\
\text { TYPE }\end{array}$ & \multicolumn{4}{|c|}{ METER VALUE } & DEVICE & STATUS & BATTERY \\
\hline $\begin{array}{c}\text { EXAMPLE } \\
\text { (HEX) }\end{array}$ & 01 & & $\odot \odot \odot$ & $01 \mathrm{D}$ & & 05 & $\odot \odot$ & 58 \\
\hline
\end{tabular}

Figura 3.36. Trama de subida de dispositivo a gateway.

La longitud de la trama es de 8 bytes (64 bits) y el formato es similar al de la trama enviada por el dispositivo de telemedida y telecontrol directamente a una red Sigfox o LoRaWAN ya descrita en la Tabla 3.6. La principal diferencia es el campo Frame Type, que dependiendo de su valor indica si se solicita una confirmación de bajada o no. Los posibles valores pueden consultarse en la Tabla 3.10 .

\begin{tabular}{|l|ll|}
\hline FRAME TYPE & DESCRIPTION: & Frame type code \\
\cline { 2 - 3 } & LENGHT: & 1 byte / 8 bits \\
\cline { 2 - 3 } & VALUES: & 1: Water meter data \\
\hline
\end{tabular}

Tabla 3.10. Descripción de la trama de subida a gateway.

En caso de enviar el dispositivo una trama de tipo 02 con solicitud de ACK, el gateway responde con una trama acorde al siguiente formato de la Figura 3.37.

\begin{tabular}{l}
\begin{tabular}{|c|c|c|c|c|c|c|c|c|}
\hline BYTE & $\mathbf{0 0}$ & $\mathbf{0 1}$ & $\mathbf{0 2}$ & $\mathbf{0 3}$ & $\mathbf{0 4}$ & $\mathbf{0 5}$ & $\mathbf{0 6}$ & $\mathbf{0 7}$ \\
\hline INF0 & $\begin{array}{c}\text { FRAME } \\
\text { TYPE }\end{array}$ & OUTPUT & $\begin{array}{c}\text { INTERVAL } \\
\text { UNITS }\end{array}$ & $\begin{array}{c}\text { TX } \\
\text { INTERVAL }\end{array}$ & \multicolumn{4}{|c|}{ TIME } \\
\hline $\begin{array}{c}\text { EXAMPLE } \\
\text { (HEX) }\end{array}$ & 01 & 00 & 01 & 07 & & $5 \mathrm{~A} 782 \mathrm{C} 37$ \\
\hline
\end{tabular} \\
\hline
\end{tabular}

Figura 3.37. Trama de bajada de gateway a dispositivo.

La longitud: 8 bytes (64 bits) y los campos presentes y su descripción es la misma que en la trama de bajada directa de red Sigfox o LoRaWAN a dispositivo de telemedida y telemedida tal y como se describió en la Tabla 3.7.

Una vez recibida una trama con información de un dispositivo final de telemedida y telecontrol, el gateway envía a su vez mediante red Sigfox una trama con el mismo formato mostrado en la 
Figura 3.36 pero siendo el campo FRAME TYPE siempre igual a 1. En función de lo requerido por los dispositivos operando a través del gateway, se solicita o no a la red Sigfox un mensaje de bajada.

El firmware se encuentra estructurado en los siguientes archivos:

- Main.pde : Funciones principales del software.

- Debug.pde : Funciones para depuración.

- LoRa.pde : Funciones para el uso del módulo radio LoRa.

- Sigfox.pde : Funciones para el uso del módulo radio Sigfox.

La carga del mismo en el dispositivo se hace con el software Waspmote Pro IDE. El procedimiento de programación del firmware se encuentra descrito en el Anexo A2.

\subsubsection{Estación base LoRaWAN}

\subsubsection{Descripción}

El dispositivo aquí descrito y que puede verse en la Figura 3.38 tiene como finalidad el hacer de puente entre una red LoRaWAN y una red IP. En la red LoRaWAN operarían los dispositivos IoT finales y en la red IP se encontraría el servidor al que se hacen llegar los datos generados por los dispositivos.

Existen en el mercado diversos dispositivos comerciales con la misma funcionalidad pero a un coste mucho mayor. En determinados casos el acabado final de otros productos es más robusto u ofrecen funciones adicionales, pero en un gran número de aplicaciones la solución aquí descrita puede ser suficiente. Se cuenta además con la ventaja de utilizar software open-source con la ventaja de poderlo mejorar, personalizar o corregir errores sin depender de terceros.

A diferencia de otras soluciones de bajo coste, el dispositivo no es un nodo LoRa con el que se establece un enlace punto a punto y que sólo admite una conexión simultánea o utiliza un protocolo de enlace propietario. En este caso, se trata de un concentrador real compatible LoRaWAN con doble radio capaz de atender 8 canales simultáneos de $125 \mathrm{kHz}$. 

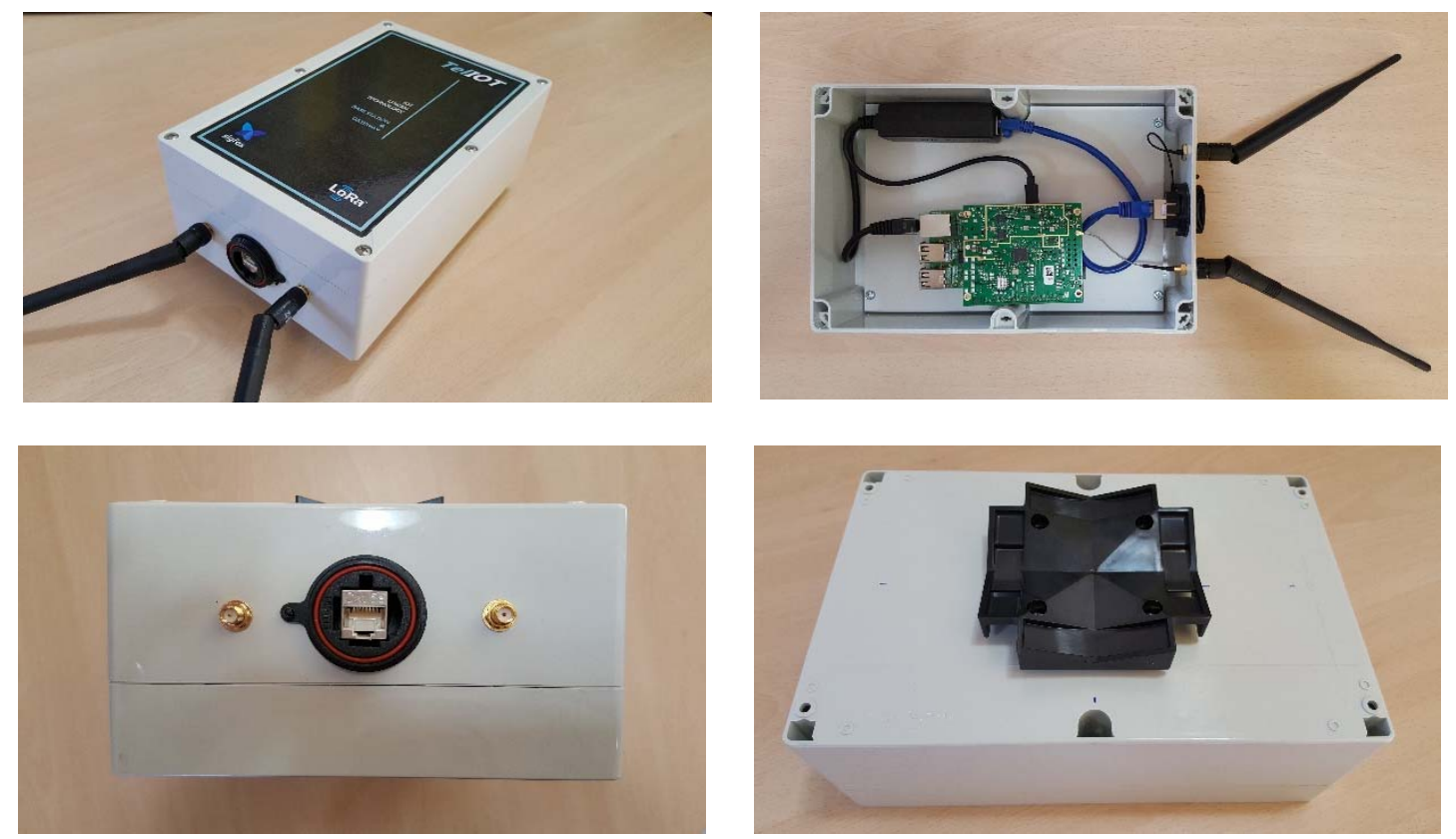

Figura 3.38. Vista exterior e interior del dispositivo gateway.

Las ventajas que ofrece del dispositivo desarrollado respecto a productos comerciales son las siguientes:

- Bajo coste.

- Software Open-Source.

- Compatible con el protocolo LoRaWAN.

- Alimentación POE.

- Carcasa IP67 con montaje en mástil.

- DDR (Ajuste dinámico de la velocidad de datos).

- Sensibilidad de recepción de hasta $-142.5 \mathrm{dBm}$.

- Capacidad de operar con SNR negativo de hasta $-9 \mathrm{~dB}$.

- 2 radios y 8 canales simultáneos de $125 \mathrm{KHz}$.

- Conectividad Sigfox opcional.

El dispositivo aquí descrito lleva a cabo la función de gateway o intermediario entre una red LoRaWAN y una red IP. A grandes rasgos puede verse la estructura seguida en la Figura 3.39.

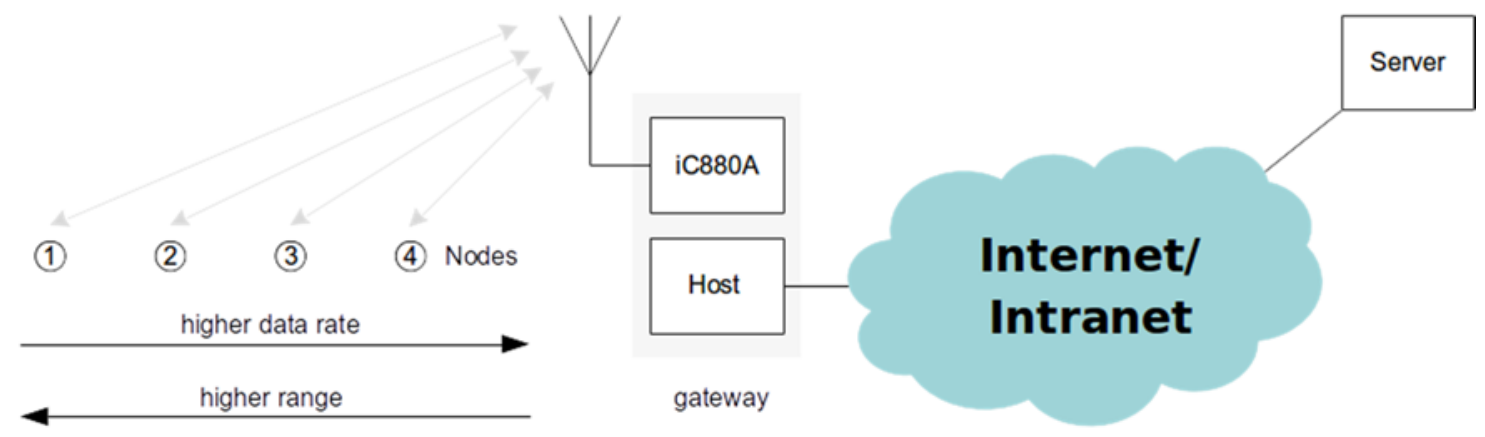

Figura 3.39. Situación del gateway en una red LoRaWAN. 
Con más detalle se distinguen los diferentes bloques funcionales que componen el gateway en el diagrama de la Figura 3.40.

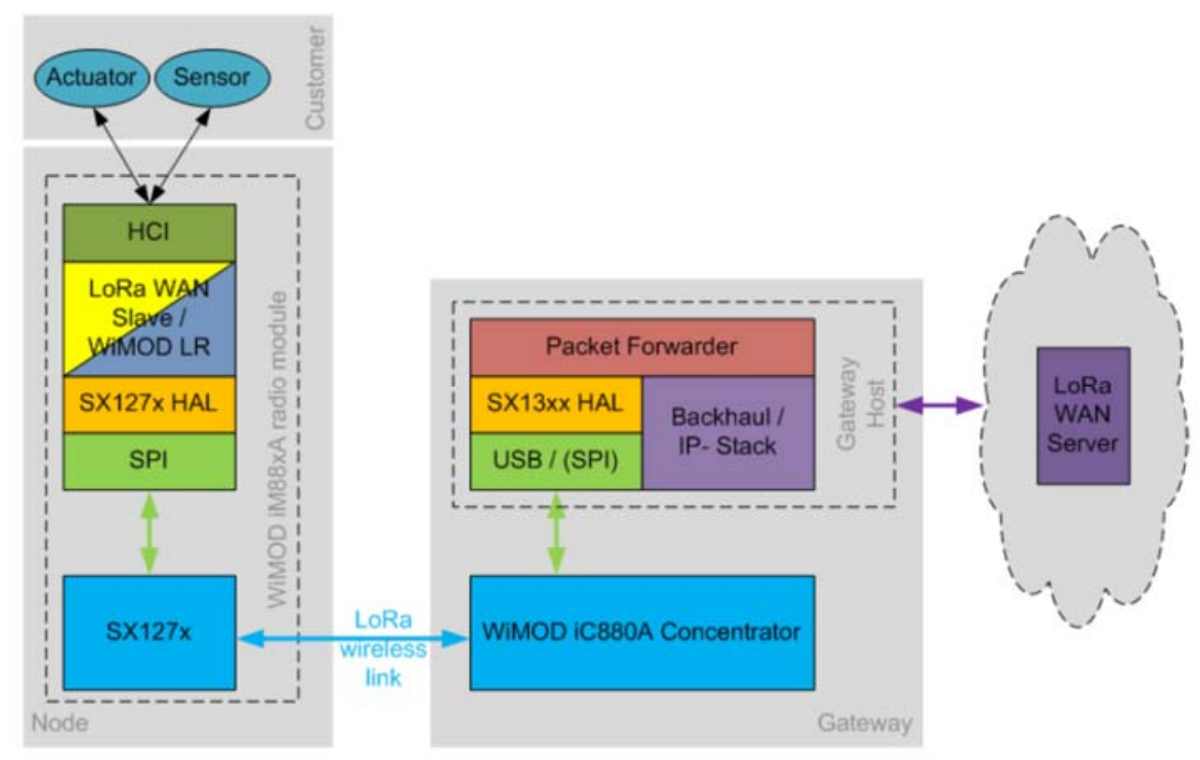

Figura 3.40. Bloques funcionales que componen el gateway.

\subsubsection{Desarrollo hardware}

Los elementos principales son la placa electrónica del concentrador que proporciona la doble radio en banda ISM 868 junto al gestor de estas, y la placa principal o host donde se ejecuta el software que hace la función de gateway y que además proporciona el hardware de red necesario para la conectividad IP vía Ethernet cableado o WiFi inalámbrico.

Los elementos empleados son los siguientes:

- Raspberry Pi 3 Model B

- Concentrador LoRa basado en SX1301 + SX1257 x2

- Placa de adaptación Concentrador-Raspberry

- Separador de tensión y datos para POE (Power Over Ethernet).

- Carcasa IP67

- Kit de montaje en mástil

- Módulo radio Sigfox

- Antena LoRa

- Antena Sigfox 


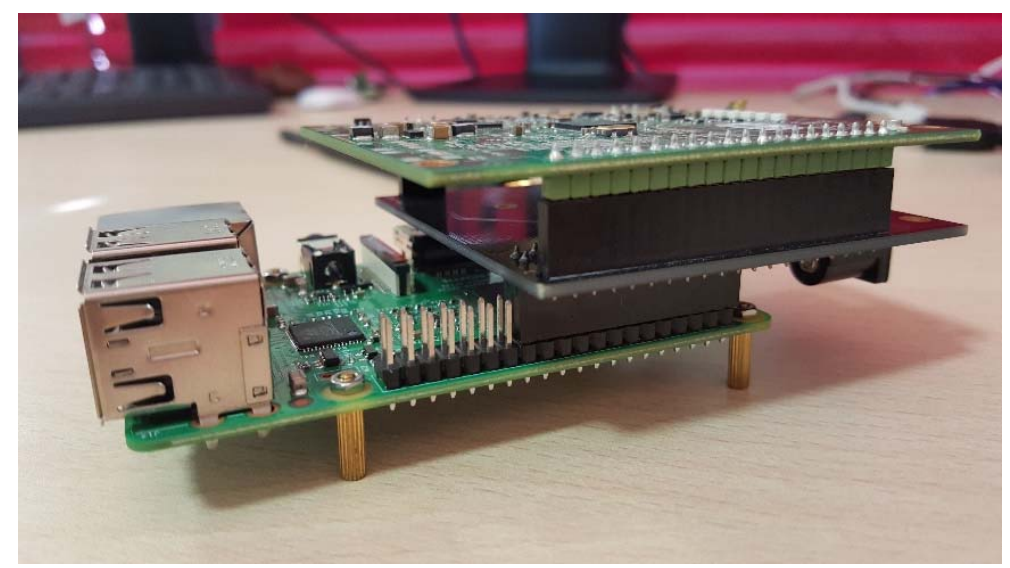

Figura 3.41. Placa base Raspberry-Pi, adaptadora y concentrador LoRaWAN.

Se puede ver la placa del concentrador sobre la placa de adaptación en la Figura 3.41 y la antena conectada al U.FL situado más cerca del conector HDMI como puede verse en la Figura 3.42 .
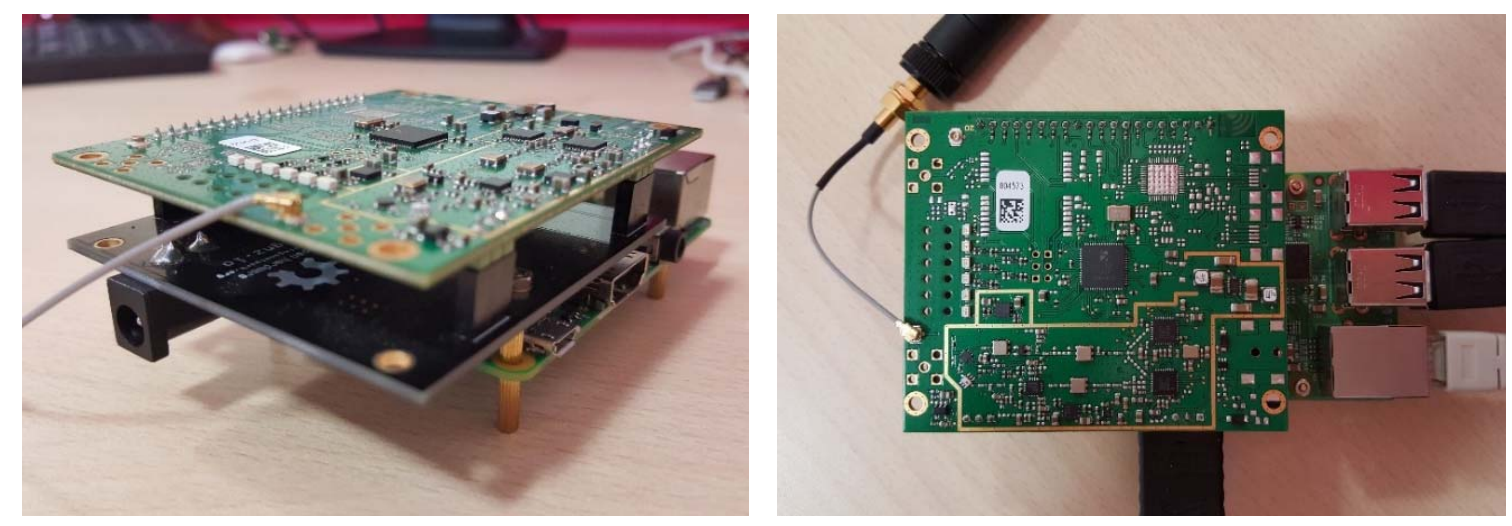

Figura 3.42. Vista del conjunto de placas y del conector de antena UFL del concentrador.

Se ha de alimentar el conjunto a través del USB de alimentación de Raspberry, no el conector de alimentación de la placa de expansión. Usar una fuente de alimentación de $2.5 \mathrm{~A}$ mínimo. Al alimentar debe iluminarse en verde el led superior (6) situando el conjunto con el conector HDMI hacia abajo.

Se puede verificar el correcto funcionamiento observando el encendido de los LEDs del concentrador LoRaWAN. Al recibir una trama LoRaWAN, se iluminarán en amarillo los leds número 4 (RX FSK packet) y número 2 (TX packet).

\subsubsection{Desarrollo software}

Como sistema operativo se utiliza Raspbian, una implementación de Linux para la placa base Raspberry-Pi. Se ha instalado a modo de driver para el chip SX1301 una librería denominada "lora_gateway que hace las veces de capa de abstracción del hardware o HAL. Esta librería es empleada por la utilidad "packet_forwarder" que recoge las tramas LoRa y reenvía sus datos hacía el servidor remoto LoRaWAN via IP. 


\subsubsection{Verificación de funcionamiento}

Para verificar el correcto funcionamiento, la unidad se ha dispuesto en una red formada por dispositivos LoRa ya probados y un servidor LoRaWAN ya funcionando. Ha pasado a sustituir al gateway de esa red que era un modelo comercial del fabricante Multitech [Multitech, 2018], en concreto la referencia MTCDTIP-LEU1-220-868-OP. En la Figura 3.43 puede verse la situación del gateway dentro de una estructura de red típica LoRaWAN.

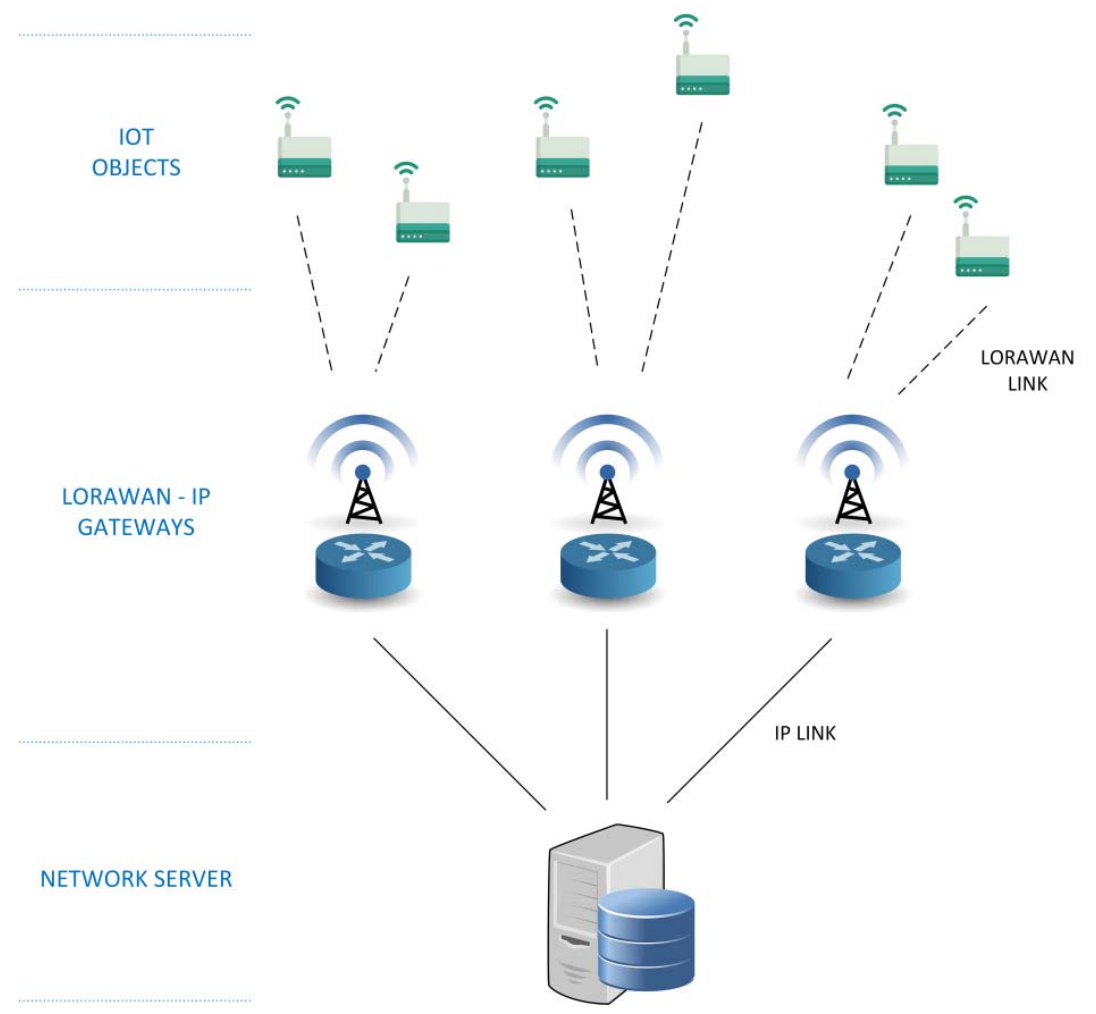

Figura 3.43. Estructura de una red LoRaWAN.

Para inyectar de manera voluntaria una trama en la red LoRaWAN y que el gateway la reenvíe al servidor, se ha utilizado el generador de señal Adeunis RF Field Test Device LoRaWAN 868 con referencia ARF8123AA.

Se ha podido comprobar a través de la pantalla del dispositivo generador, que tras el envío de una trama se ha generado otra de bajada con confirmación y de la que podemos ver el nivel de señal RF. Si la trama generada no hubiese realizado correctamente todo el trayecto hasta el servidor y de vuelta a través de nuestro gateway, no se hubiese visualizado la información de bajada como se aprecia en la Figura 3.44.

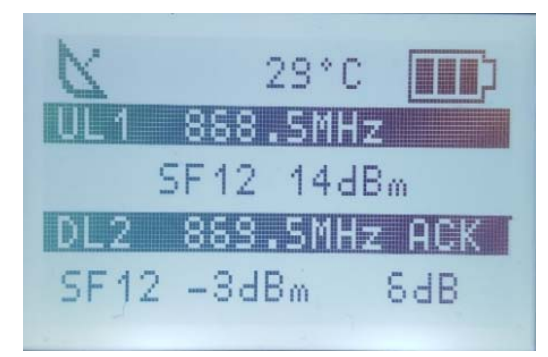

Figura 3.44. Pantalla del emulador de nodo LoRa tras enviar la trama. 


\subsubsection{Dispositivo para el estudio de la cobertura}

\subsubsection{Descripción}

En el momento de abordar despliegues que impliquen comunicaciones a través de redes loT de tipo LPWAN, se hace necesario conocer si la cobertura de la red en determinadas localizaciones es suficiente para poder establecer la comunicación.

Los dispositivos comercialmente disponibles para cubrir esta necesidad únicamente son capaces de enviar una trama a través de un único tipo de red LPWAN para la que están diseñados. En determinados casos son capaces de mostrar la señal en $\mathrm{dBm}$ con la que se recibe la trama de bajada pero su contenido sólo si se trata de caracteres ASCII imprimibles. Las opciones de configuración son muy reducidas y se hace necesario una conexión USB con un PC y el envío de comandos AT. Además, al tratarse de hardware y software propietario y cerrado, las posibilidades de personalización son muy limitadas.

Con objeto de superar estas limitaciones se ha diseñado y construido el dispositivo electrónico de bajo coste a continuación descrito. Se trata de un producto que a diferencia de los disponibles comercialmente, es capaz de comunicación loT tanto Sigfox como LoRaWAN. Dispone de una interface de usuario mediante pantalla TFT táctil en la que presentar la información y modificar la configuración. Puede establecer una conexión de red WiFi con Internet, o actuar como punto de acceso permitiendo la conexión punto a punto con un dispositivo móvil. Además es capaz de recoger y almacenar el nivel de señal devuelto por la red y los datos adquiridos se pueden ir almacenando en una tarjeta de memoria extraíble.

No hay que restar importancia al hecho de que al tratarse de un desarrollo propio, este puede mejorarse, personalizarse según aplicación y admitir futuras ampliaciones. En la Figura 3.45 puede verse el resultado final con y sin la instalación de pantalla para dejar al descubierto los elementos bajo esta.
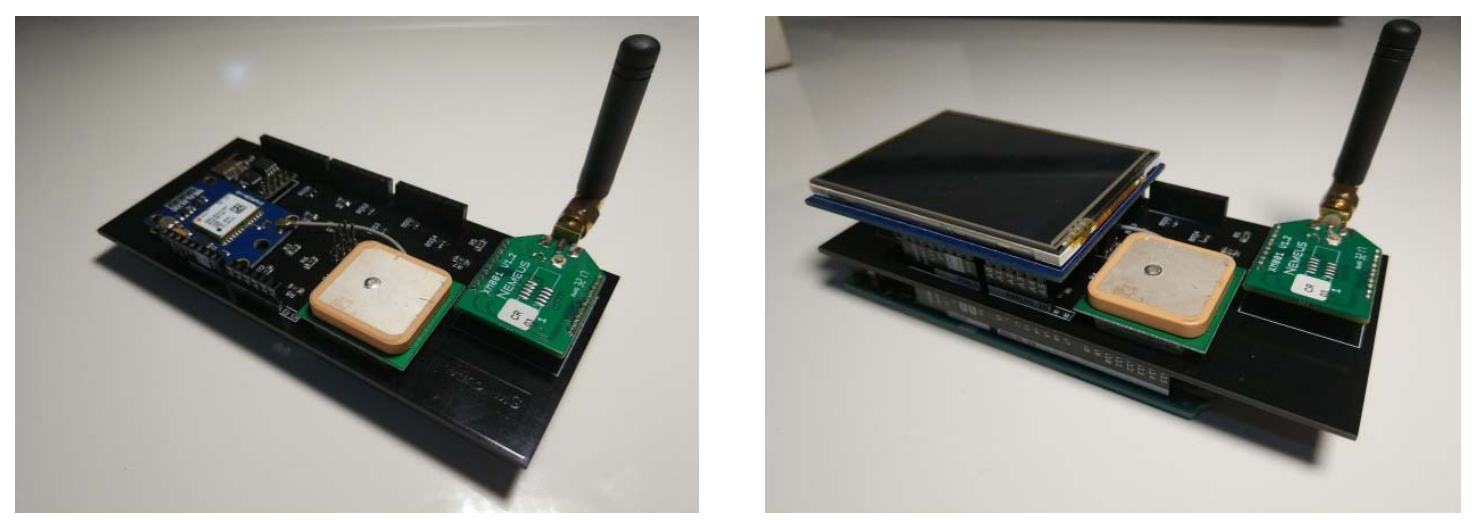

Figura 3.45. Imágenes del dispositivo desarrollado para estudio de cobertura.

\subsubsection{Desarrollo hardware}

Se ha llevado a cabo el desarrollo de circuitería para cubrir la necesidad de interconexión de los diferentes módulos periféricos con la placa de control y con objeto también de conseguir que el conjunto quede compacto y solidario. Para ello, dado que la placa de control utilizada incluye conectores de ampliación para admitir placas de expansión, se ha diseñado una placa de circuito 
impreso que pueda ser insertada sobre la de control y a su vez sobre ella, los diferentes módulos de comunicaciones y pantalla. De manera, el conjunto queda como una sola unidad compacta y solidaria sin cableado externo. En la Figura 3.46 puede verse un diagrama de bloques de conjunto.

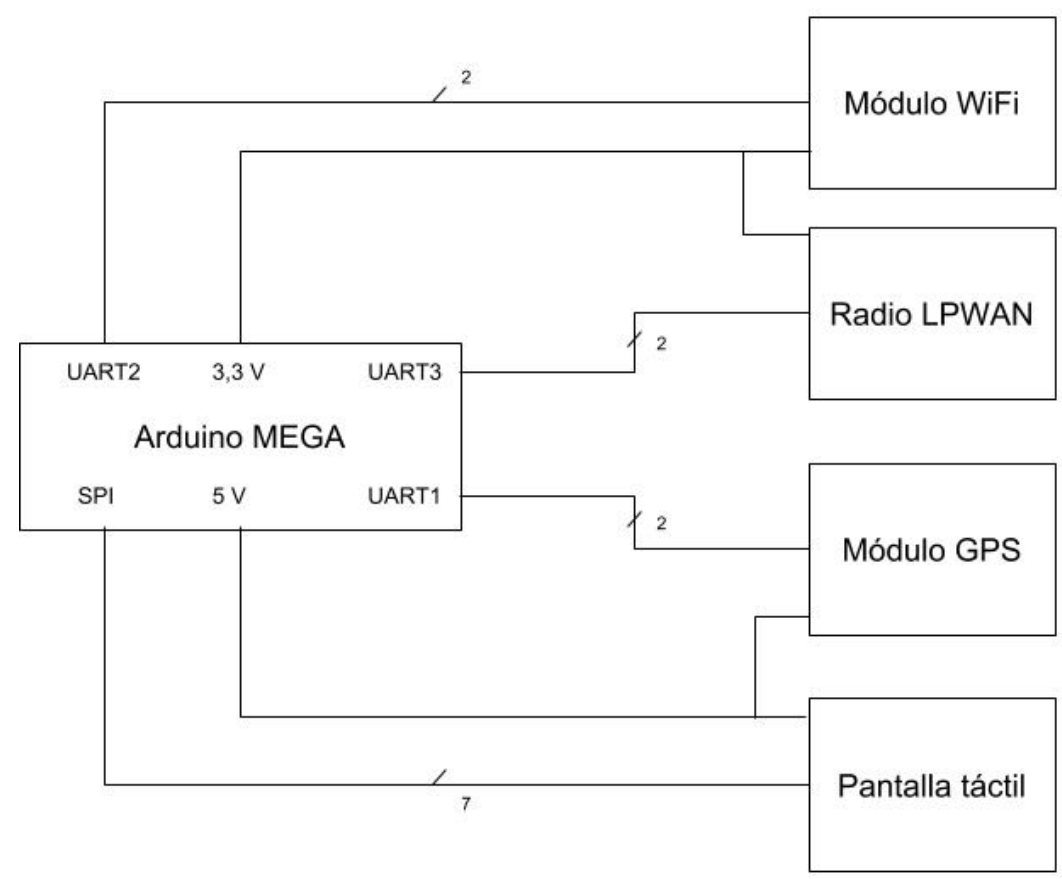

Figura 3.46. Diagrama de bloques del dispositivo para estudio de cobertura.

Los elementos hardware utilizados son:

- Unidad de control Arduino Mega: Se ha basado el diseño en esta placa por tratarse de hardware y software abierto. Trabaja a una tensión de $5 \mathrm{~V}$ y su programación se realiza mediante puerto USB. Dispone de una gran cantidad de puertos de ampliación permiten la conexión de los demás periféricos. Respecto al modelo más popular Arduino UNO, esta placa ofrece una mayor cantidad de memoria de programa, necesaria para esta aplicación y para permitir ampliaciones futuras. Además, el disponer de más de un puerto serie UART posibilita la conexión simultanea de los diferentes módulos a continuación descritos.

- Módulo GPS NEO6MV2: Se encarga de proporcionar la posición global del dispositivo en función de la señal recibida de la red de satélites GPS (Global Position System). La comunicación es a través de un puerto serie UART. Se alimenta a 5V. Incluye una antena de dimensiones $25 \times 25 \mathrm{~mm}$ y las dimensiones totales del módulo son $25 \times 35 \mathrm{~mm}$.

- Radio XM001-EU. Facilita las comunicaciones LPWAN en banda ISM $868 \mathrm{MHz}$ tanto en Sigfox como en LoRaWAN. Integra el módulo MM002-EU en formato XBEE que destaca por su bajo consumo e incluye un conector SMA para una antena externa y con ello aumentar la distancia de enlace. Se alimenta a 3.3V. 
- Módulo WiFi: Está basado en el SoC (System on Chip) ESP8266 capaz de conectar a redes inalámbricas $802.11 \mathrm{~g}$. Admite ser programado con código para trabajar de forma autónoma, aunque esta funcionalidad no ha sido utilizada en esta aplicación.

- Pantalla: Se trata de una pantalla TFT de 2.8" con resolución $240 \times 320$ pixels e interface táctil resistiva. Incluye además un lector de tarjetas $\mu \mathrm{SD}$. La tensión de operación es de $5 \mathrm{~V}$ por lo que se puede conectar directamente a la placa de control. Utiliza el interface de comunicaciones SPI.

Dado que los puertos de entrada y salida de placa de control, incluida la UART, trabajan a una tensión de 5V mientras que los módulos LPWAN y WiFi a 3.3V, se hace necesaria una adaptación bidireccional de niveles en las líneas de comunicación que conectan estos elementos. Para ello se han usado transistores MOSFET de canal N con referencia BSS138.

En la Figura 3.47 puede verse un esquemático eléctrico con la interconexión de los diferentes elementos.

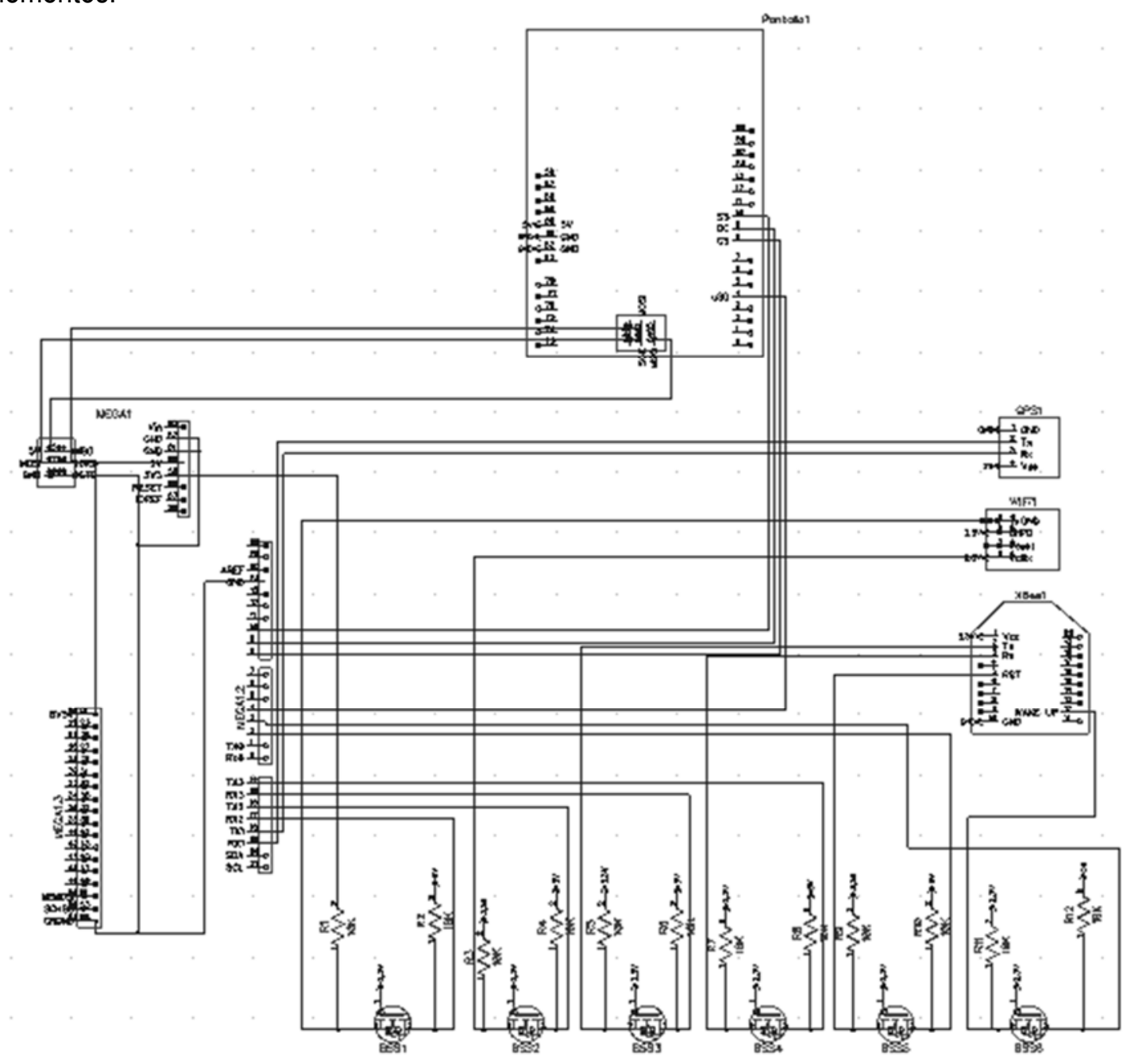

Figura 3.47. Esquemático eléctrico del dispositivo para estudio de cobertura.

En la Figura 3.48 se muestra la placa de circuito impreso en la fase de diseño y ya finalizada. Se trata de una placa doble cara con componentes SMD de montaje superficial, ancho mínimo de pista de $0.3 \mathrm{~mm}$ y acabado en barniz negro. 

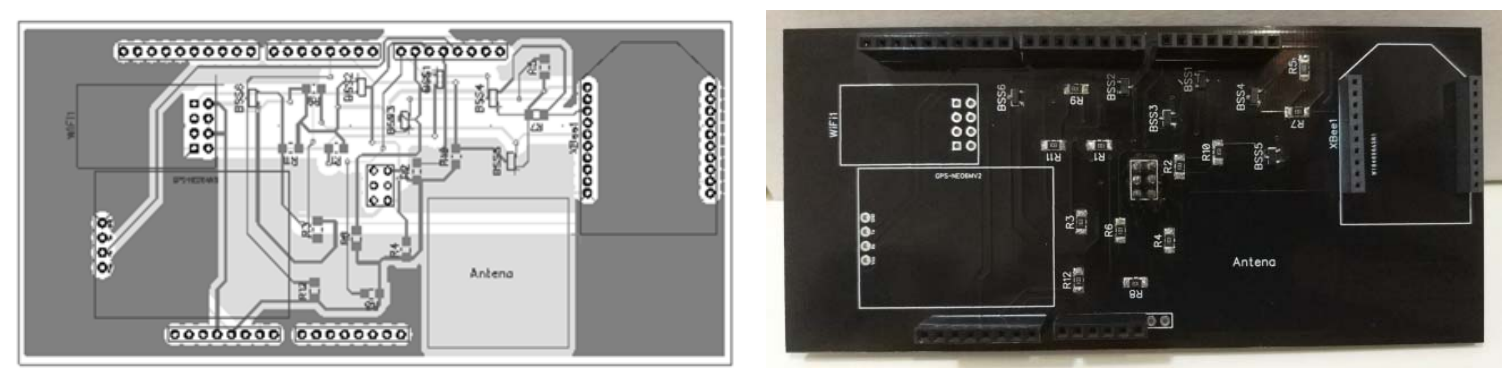

Figura 3.48. Placa de circuito impreso diseñada para el dispositivo para estudio de cobertura.

En la Figura 3.49 puede verse la unidad instalada en un vehículo para una sesión de pruebas de campo. Al ser la tensión de alimentación de $5 \mathrm{~V}$ se hace posible el empleo de una batería externa de tipo "Powerbank" de 2200 mAh y salida USB.

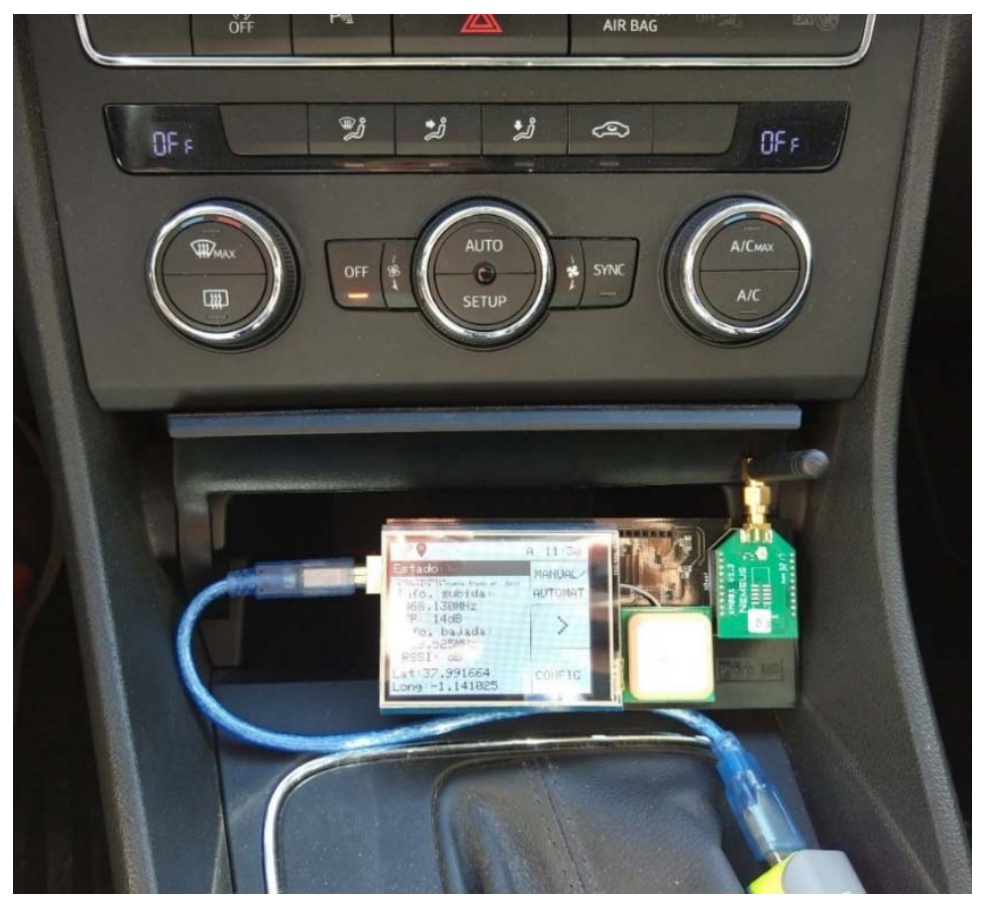

Figura 3.49. Imagen del dispositivo instalado en vehículo durante sesión de pruebas.

\subsubsection{Desarrollo software}

El software que se ha desarrollado para el microcontrolador ATmega de la placa de control lleva a cabo la gestión simultanea de los diferentes periféricos hardware como la pantalla táctil, la memoria externa $\mu S D$, el módulo de comunicaciones LPWAN, el módulo de comunicaciones WiFi y el receptor GPS. En el caso del módulo LPWAN se ha implementado la gestión directamente a través de la interface serie mediante comandos AT sin utilizar ninguna librería intermediaria. En la Figura 3.50 puede verse el diagrama de flujo de operación. 


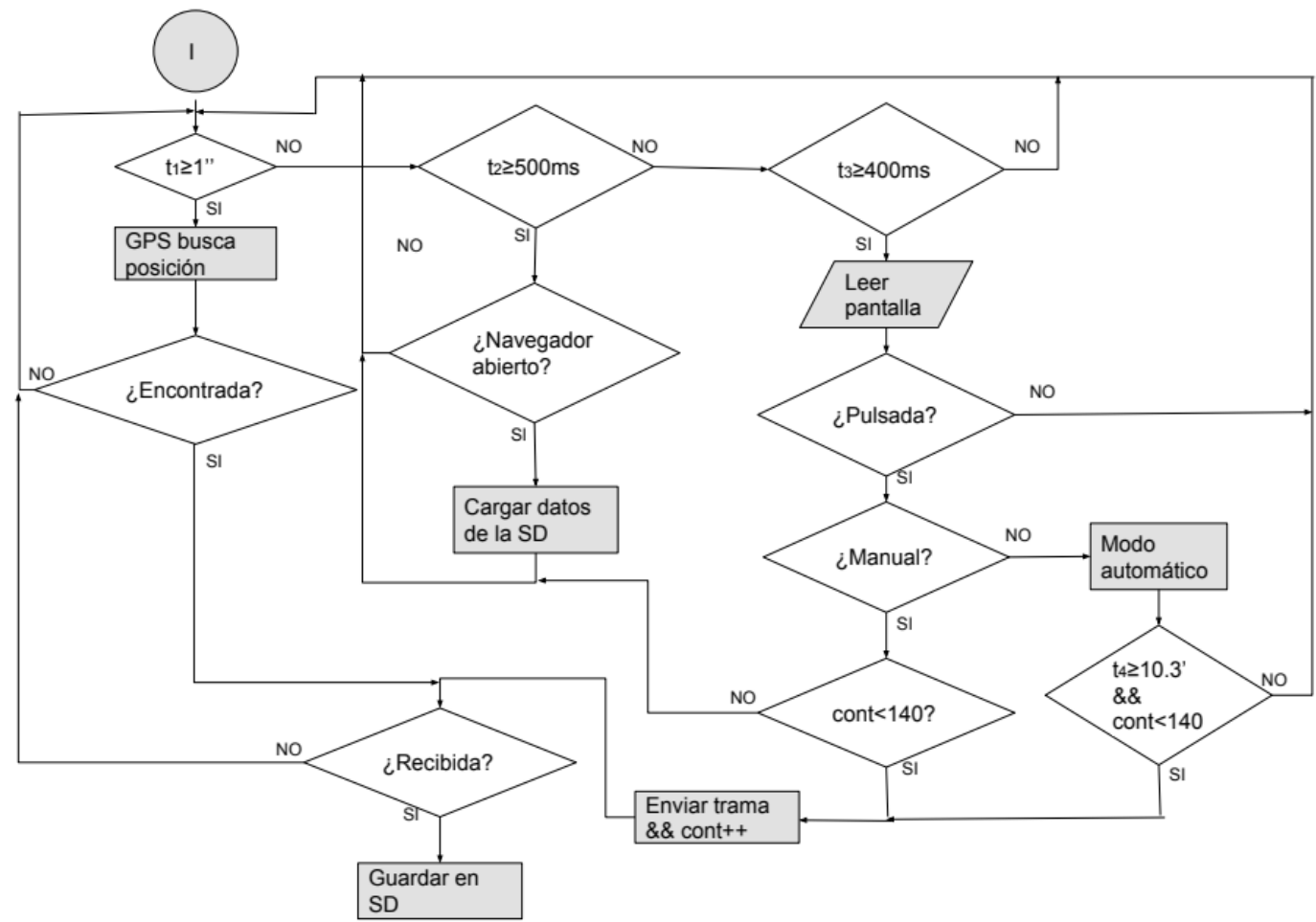

Figura 3.50. Flujograma del software embebido.

En la Figura 3.51 puede verse una imagen de la pantalla con la interface de usuario. En la parte superior se encuentra la barra de estado informando entre otras cosas del estado de las comunicaciones. La parte derecha comprende los pulsadores virtuales para la operación y configuración. En la parte izquierda se muestra los datos de la operación en curso.

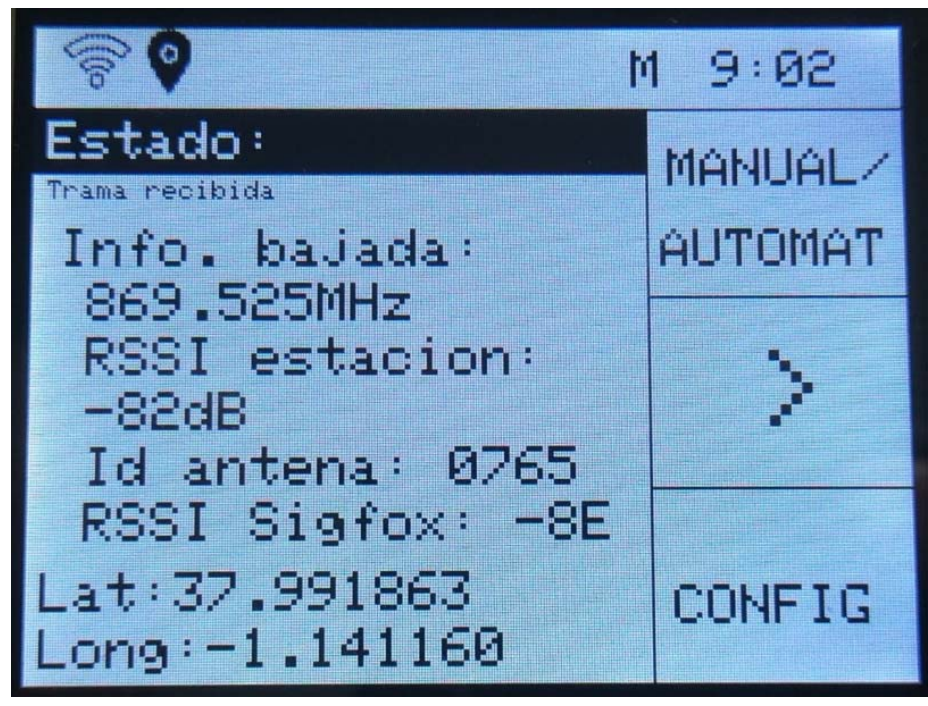

Figura 3.51. Interface de usuario. 
Los datos adquiridos se almacenan en un fichero de texto con formato de valores separados por comas CSV. Cada línea comprende una muestra e incluye: fecha, hora, latitud, longitud, rssi de la señal de la estación base, identificador de la estación base y rssi de nuestra señal recibida por la estación base. Puede verse una muestra de los datos generados en la Figura 3.52.

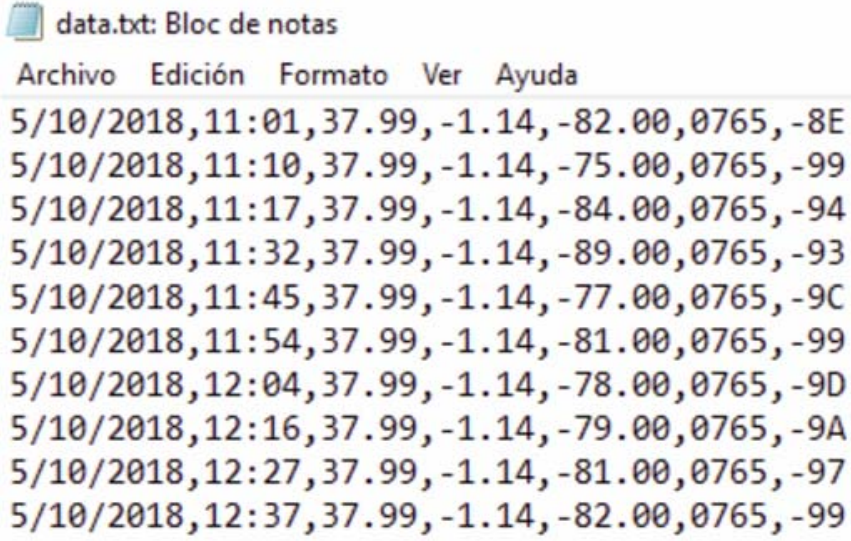

Figura 3.52. Ejemplo de datos descargados. 


\subsection{Implementación software}

\subsubsection{Descripción}

La arquitectura de red loT mixta aquí propuesta consta de una serie de elementos software para dirección, acondicionar y almacenar los datos procedentes de los dispositivos loT. Estos elementos y la relación entre ellos se encuentran representados en la Figura 3.53 indicándose el sentido del flujo en la adquisición de datos por parte de los diferentes elementos.

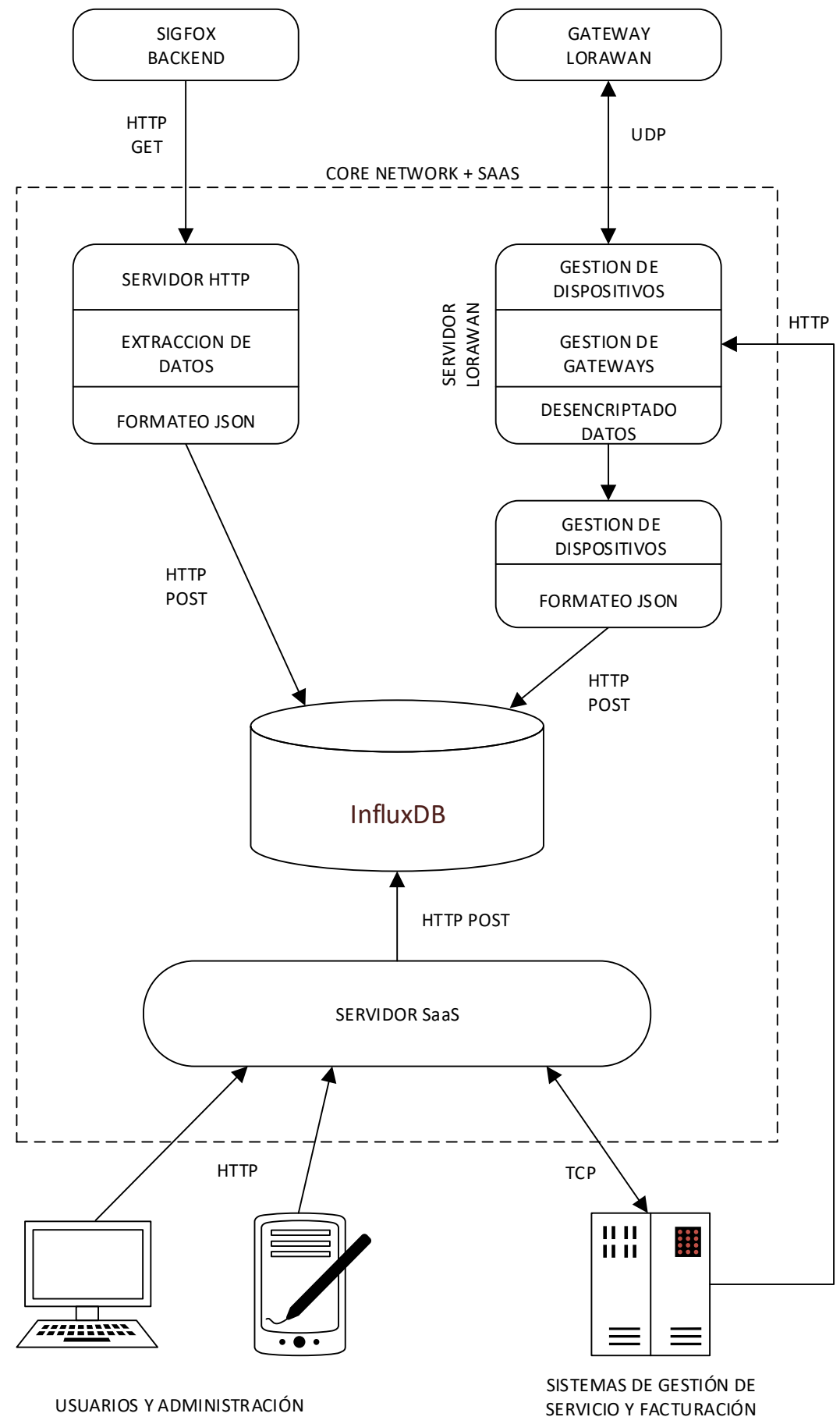

Figura 3.53. Estructura software de la arquitectura propuesta. 
Existen dos caminos principales de llegada de información en función de si el dispositivo que la origina está conectado a la red Sigfox o a una red LoRaWAN. En el caso de Sigfox, cuando se reciben nuevos datos de un dispositivo, el Backend de la red ha sido configurado para llevar a cabo un callback generando una llamada con protocolo HTTP/GET a nuestro servidor propio con función de core network. En esta llamada va tanto la carga útil de datos, como información adicional de red consistente en el nivel de señal radio, la relación señal-ruido y el identificador de la estación base que recibió la trama, lo cual puede ser útil para la mejora de la red y la detección de errores. Un bloque funcional en el servidor propio lleva a cabo la función de intermediario entre el backend de Sigfox y la base de datos. Ha sido implementado en Python y se encarga en primer lugar de ofrecer un servicio HTTP externo para recibir las llamadas del Backend de Sigfox. Estas se efectúan con un método GET, por lo que cuando son recibidas, lo siguiente que se lleva a cabo es una extracción de la URL de los pares de cadenas identificadorvalor y a continuación un formateo de los datos en JSON para su inserción en la base de datos con una llamada HTTP/POST local.

En el caso de que los datos provengan de dispositivos conectados a una red LoRaWAN, la correspondiente estación base que ha recibido la transmisión hace la función de gateway de radio a IP y lleva a cabo el envío de la misma mediante IP/UDP al Core Network propio. Se ha utilizado LoRa-Server por ser una implementación de código abierto del protocolo LoRaWAN y ofrecer posibilidades de ampliación y personalización. Además de recibir la información se encarga de la gestión de los dispositivos manejando el registro, autentificación y encriptación del tráfico de los mismos. Así mismo lleva a cabo tareas de monitorización y optimización de enlaces por dispositivo. En función de la calidad del enlace puede solicitar a los dispositivos reajustes en la velocidad de transmisión y el ancho de banda mediante mecanismos adaptativos del protocolo LoRaWAN. Al respecto de las tramas recibidas, verifica su integridad y descarta duplicados en el caso de que varios gateways reciban al mismo dispositivo final. Se encarga también de la gestión de gateways, manteniendo una base de datos con los registrados en esa red y seleccionando el más adecuado en función del nivel de señal con el que se ha recibido al dispositivo, utilizándolo entonces para transmitir la información de bajada. De los gateway se reciben también datos adicionales del enlace como el nivel de señal RSSI con la que se ha recibido la trama, la frecuencia radio empleada y la tasa de bits. Esta información se registra también en la base de datos a efectos de estudio del rendimiento de la red y detección de errores. La conexión entre LoRa-Server y la base de datos se lleva a cabo mediante llamadas locales de tipo HTTP/POST.

Se ha utilizado InfluxDB [InfluxDB, 2018] como base de datos de código abierto, desarrollada por InfluxData en lenguaje Go y optimizada para un almacenamiento rápido y una alta disponibilidad de los datos, los cuales pueden ser obtenidos en campos de series temporales. LoRa-Server permite una integración directa con ella. Además, InfluxDB es compatible R, el lenguaje de referencia en el mundo de la estadística, lo que la hace adecuada para implementar técnicas de Big-Data e inteligencia artificial en el posterior procesado de la información recopilada. Además incluye soporte de Graphite [Graphite, 2018], una herramienta de código abierto para monitorizar y graficar series temporales de datos.

El servidor LoRaWAN dispone de una interface web desde la que registrar nuevos gateway y dispositivos, así como organizar estos por aplicación y perfil, gestionar usuarios, organizaciones y servicios. En la Figura 3.54 puede verse un organigrama de las diferentes entidades y su relación. 


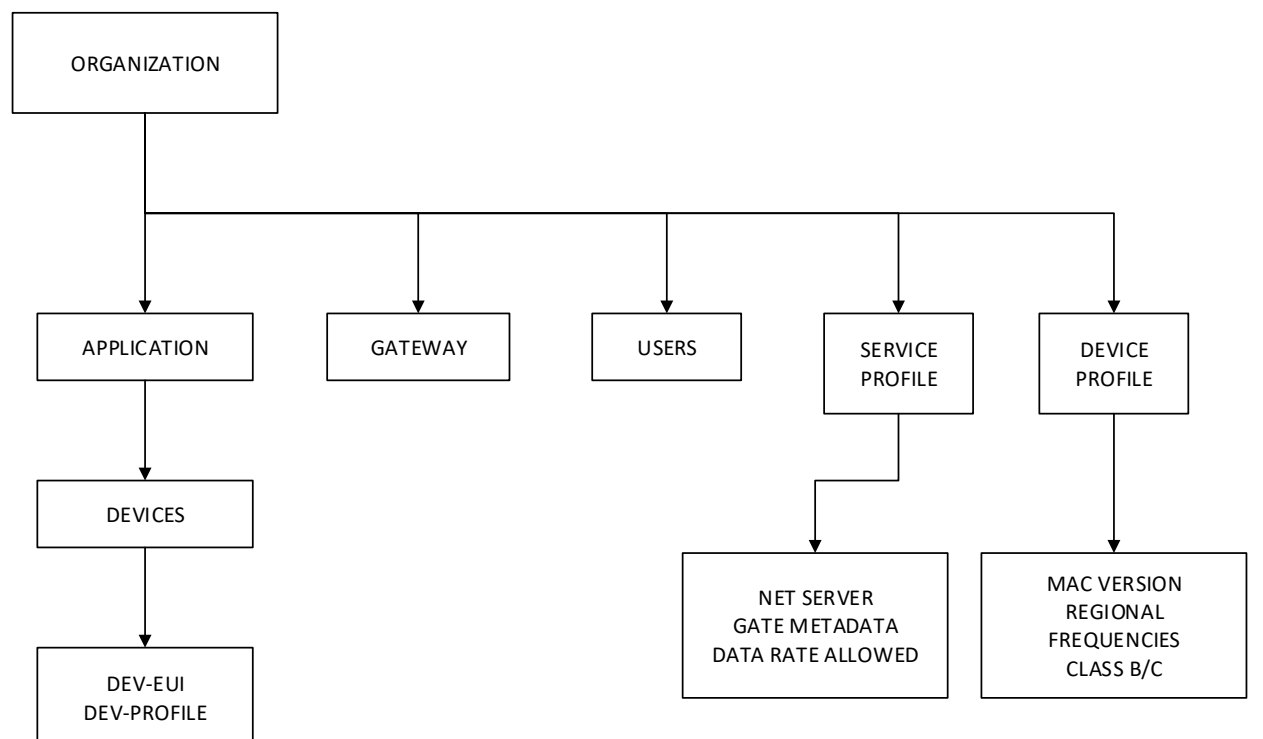

Figura 3.54. Organigrama de las diferentes entidades del servidor LoRaWAN.

A continuación, en la Figura 3.55 puede verse como se han organizado siguiendo la estructura del organigrama, los elementos para una aplicación concreta. Se ha creado una organización de nombre Dintel y a ella pertenecen tanto el gateway Multitech como la aplicación Teliot en la que está definido el dispositivo demostrador de Adeunis. Se ha declarado un usuario admin, un servicio por defecto y dos perfiles de dispositivo tanto para autentificación OTAA como ABP.

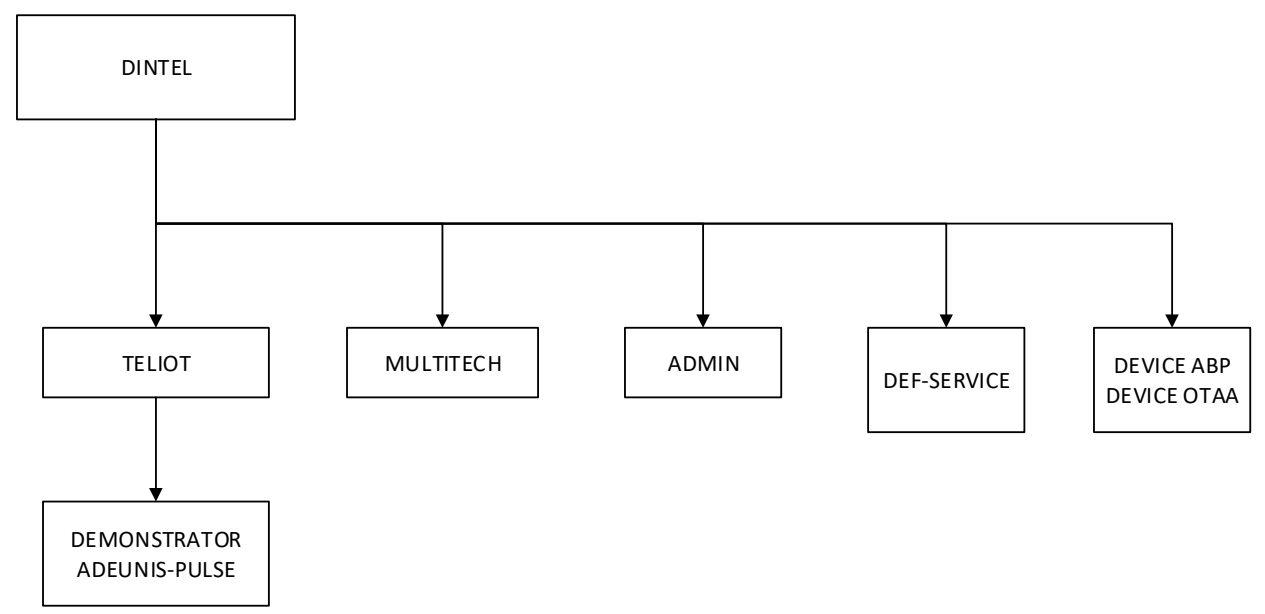

Figura 3.55. Organigrama de las diferentes entidades del servidor LoRaWAN.

La secuencia para hacer operativo el servidor consiste en crear y nombrar una organización. A continuación se declaran los gateways pertenecientes a esa organización indicando su dirección MAC como puede verse en la Figura 3.56. 


\section{LoRa Server}

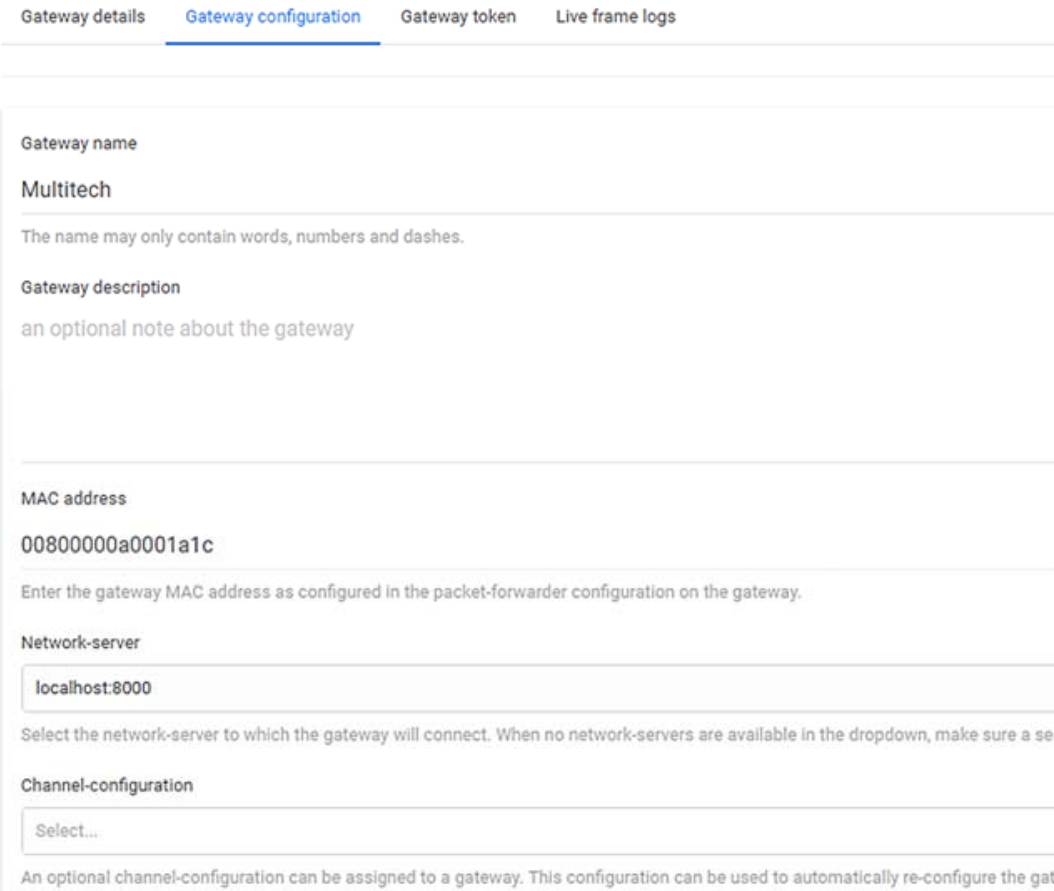

Figura 3.56. Declaración de gateways en el servidor LoRaWAN.

A continuación se crean los usuarios definiendo sus permisos y también los perfiles de dispositivos. En los perfiles de dispositivo es donde se ha de elegir si la seguridad será de tipo JOIN o ABP como puede verse en la Figura 3.57.

\section{LoRa Server}

Organizations / LoRa Server

Applications Gateways Organization configuration Organization users Service profiles Device profiles

Update device-profile

General Join (OTAA / ABP) Class-B Class-C

Device-profile name

Device ABP

A memorable name for the device-profile.

Network-server

localhost: 8000

The network-server on which this device-profile will be provisioned. After creating the device-profile, this value can't be changed.

LORAWAN MAC version

Select.

Version of the LoRaWAN supported by the End-Device.

Figura 3.57. Edición de perfiles de dispositivo en el servidor LoRaWAN. 
Por último dependiente de la organización se crea una aplicación y como pertenecientes a la aplicación se añaden nuevos dispositivos indicando su identificador de dispositivo o Dev-EUI, su clave de aplicación o App-Key y el perfil al que pertenecerán como puede verse en la Figura 3.58 .

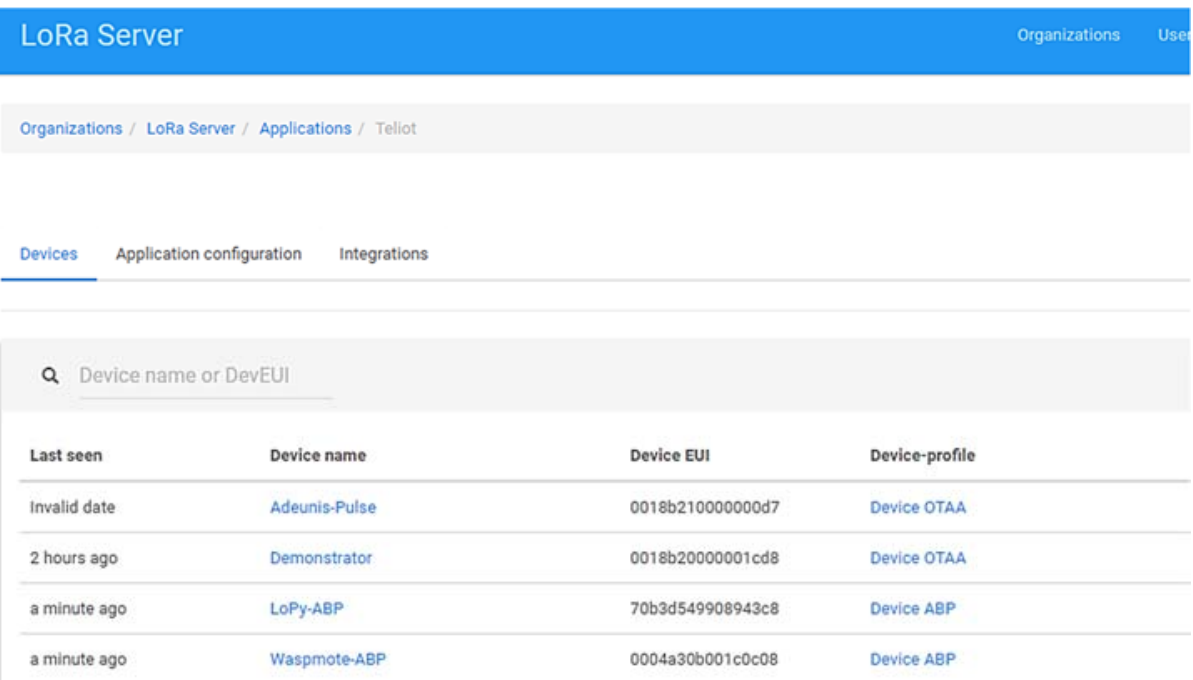

Figura 3.58. Declaración de dispositivos en el servidor LoRaWAN.

El conjunto software aquí descrito se ejecuta sobre sistema operativo Ubuntu versión 16.01 corriendo en una máquina con procesador Intel i7, 8 GB de memoria RAM y 1TB de almacenamiento en disco duro de estado sólido.

\subsection{Entorno de validación en laboratorio}

\subsubsection{Descripción}

Un contador preparado para tele-lectura dispone de una sola salida de impulsos a conectar a un dispositivo de registro o de envío remoto de la información. En el caso de querer enviar la misma información a través de varios dispositivos con fines comparativos entre estos, o de hacer llegar los datos a través de varias redes loT, se hace necesario disponer de un módulo electrónico con una entrada para contador y varias salidas para dispositivos en las que se replican los impulsos de la entrada.

Un emulador de contador genera los mismos impulsos que este produciría a diferentes caudales que son seleccionables mediante un interface de usuario implementado en una pantalla táctil. La selección entre la entrada externa de contador y el emulador se realiza mediante un conmutador. Diferentes dispositivos de envío de información a través de redes loT se reúnen a fines comparativos entre fabricantes y de validación de los propios creados en este proyecto. Así mismo, el uso simultaneo de dispositivos para diferentes redes loT tales como Sigfox y LoRaWAN permite comparar funcionalidad y rendimiento de estas.

Para dar soporte a todos estos elementos y los correspondientes conectores, se construye la consola aquí descrita y que puede verse en la Figura 3.59. 
La consola loT consta de un bastidor de aluminio en el que se ha fijado un carril DIN. Sobre el carril DIN se ubican los siguientes elementos:

- Transceptor Sigfox para contador de AdeunisRF.

- Transceptor LoRaWAN para contador de AdeunisRF.

- Transceptor WmBus para contador de AdeunisRF.

- Transceptor para sensores de tensión y corriente de AdeunisRF.

- Replicador de pulsos TelloT.

- Emulador de contador TelloT.

Las tapas laterales de plástico del bastidor se han mecanizado para ubicar los siguientes elementos:

- Conector de panel mini-usb para alimentación a 5V del emulador de contador.

- Conector de panel macho de 2 polos para entrada de pulsos de contador.

- Conmutador para selección de entrada externa o proveniente del emulador.

- Tres conectores de panel hembra de 2 polos para salidas de pulsos.

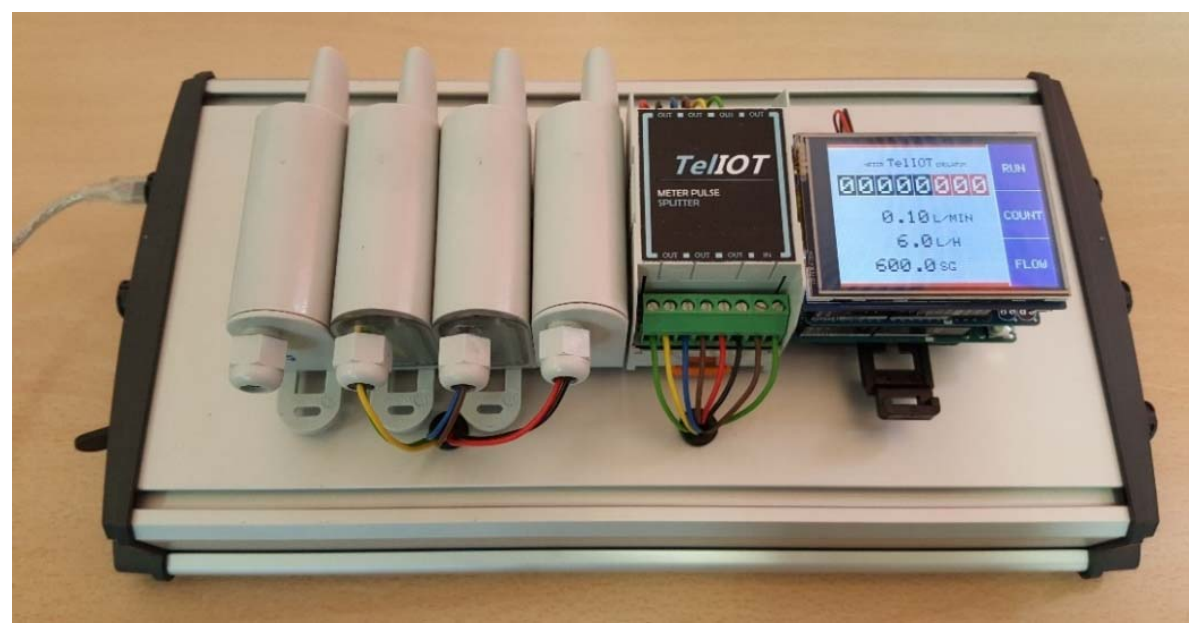

Figura 3.59. Consola para dispositivos loT.

Los elementos sobre el panel de la consola loT siguen la distribución de la Figura 3.60:

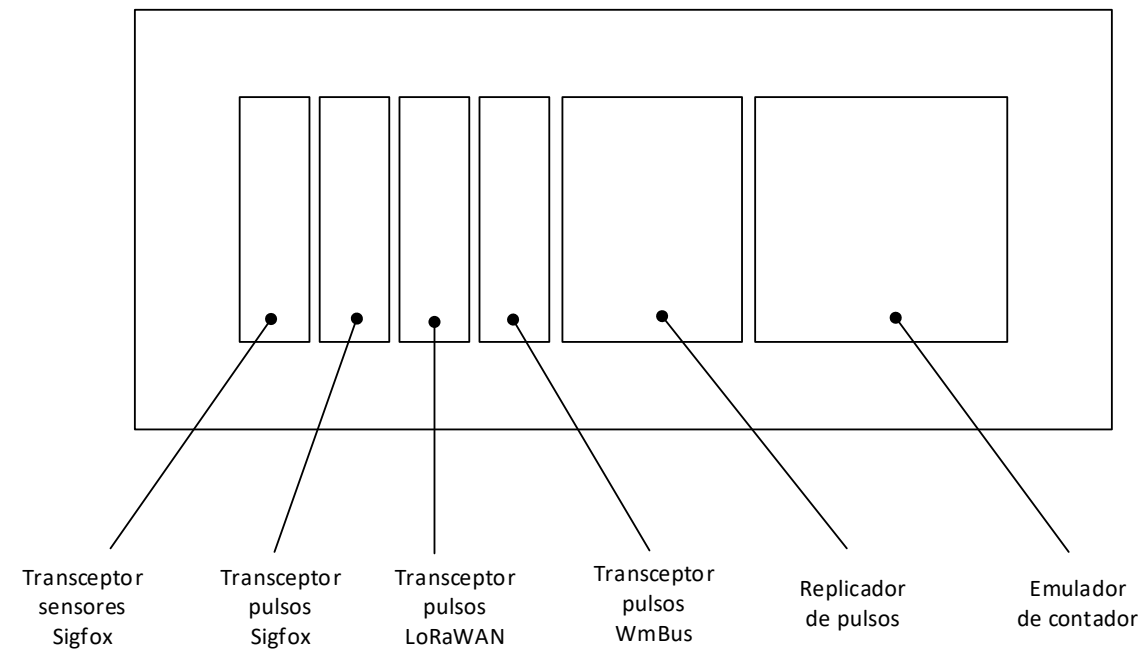

Figura 3.60. Elementos en la consola. 
La consola dispone de los siguientes conectores mostrados en la Figura 3.61.

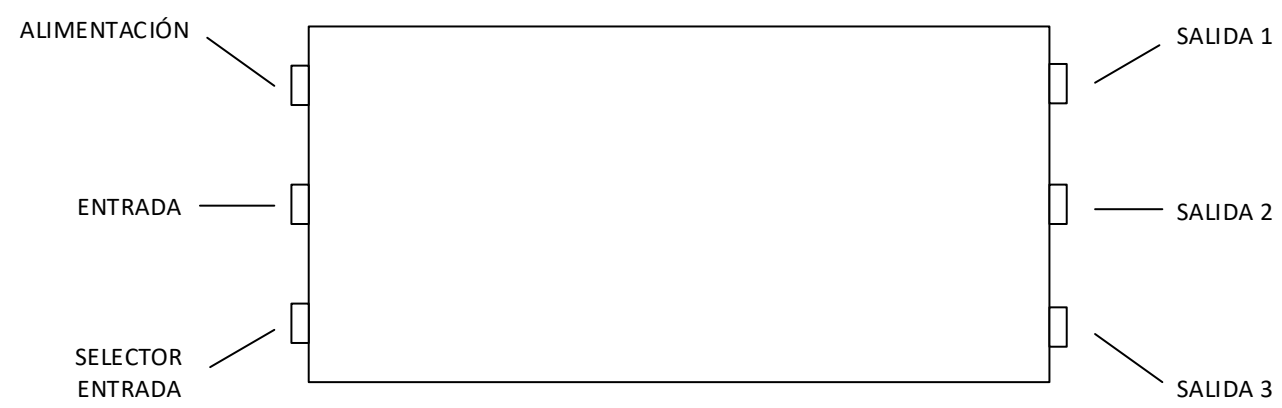

Figura 3.61. Distribución de conectores de la consola.

Alimentación: Entrada de tensión de 5V/1A de tipo micro-usb requerida para el funcionamiento del emulador de contador. Si este no se utiliza, no es necesario alimentar la consola para el resto de funciones.

Entrada: Pulsos provenientes de un contador externo. La salida ha de ser libre de potencial.

Selector entrada: Conmuta entre los pulsos provenientes de la entrada externa (posición superior) o del emulador de contador integrado (posición inferior).

Salidas 1, 2 3: Pulsos disponibles para dispositivos loT externos. Son salidas libres de potencial.

Los diferentes elementos que componen la consola se relacionan entre sí según el siguiente diagrama de bloques de la Figura 3.62.

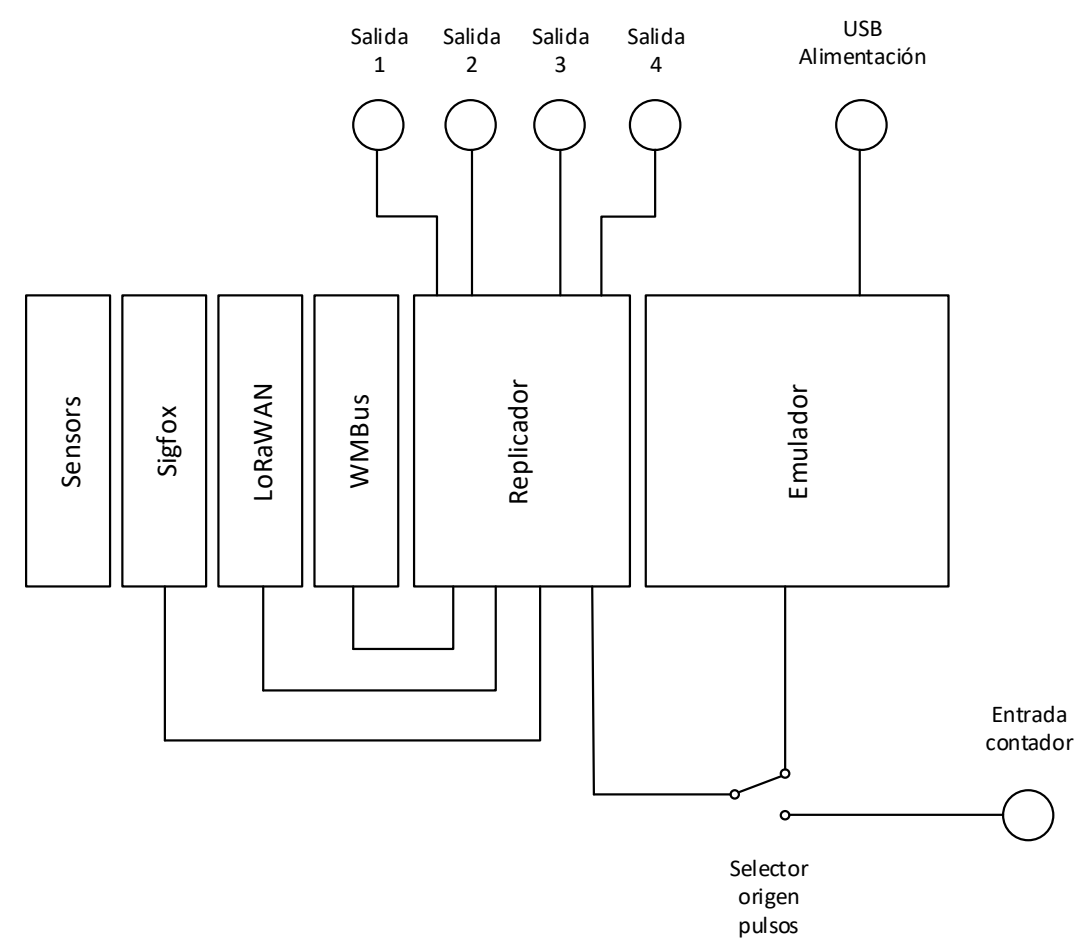

Figura 3.62. Diagrama de bloques. 


\subsubsection{Puesta en marcha}

El conexionado de cables a los módulos loT ha de hacerse según el siguiente diagrama en la Figura 3.63.
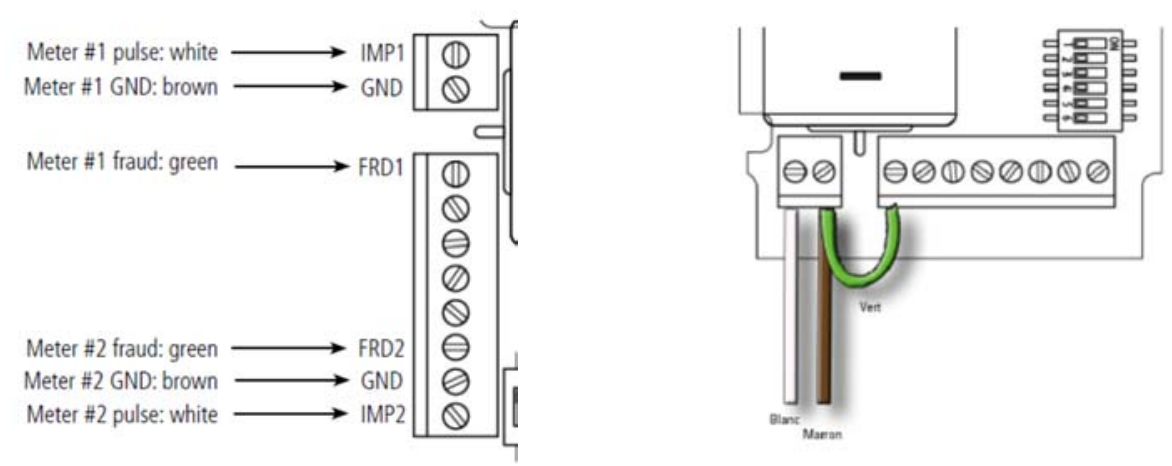

Figura 3.63. Conexionado.

La configuración según los micro-interruptores ofrece las posibilidades de la Tabla 3.11.

\begin{tabular}{|c|l|c|c|c|c|c|c|}
\hline Parámetro & \multicolumn{1}{|c|}{ Valor } & SW1 & SW2 & SW3 & SW4 & SW5 & SW6 \\
\hline \multirow{4}{*}{ Tipo de lectura } & Electricity & ON & OFF & & & & \\
\cline { 2 - 8 } & Water & OFF & OFF & & & & \\
\cline { 2 - 8 } & Gas & OFF & ON & & & & \\
\cline { 2 - 8 } & Heat & ON & ON & & & & \\
\hline \multirow{3}{*}{ Unidades por pulso } & $1 \mathrm{~L} / 10 \mathrm{dm}^{3} / 1 \mathrm{Wh}$ & & & OFF & OFF & & \\
\cline { 2 - 8 } & $10 \mathrm{~L} / 10 \mathrm{dm}^{3} / 10 \mathrm{Wh}$ & & & OFF & ON & & \\
\cline { 2 - 8 } & $100 \mathrm{~L} / 100 \mathrm{dm}^{3} /$ & & & ON & OFF & & \\
\cline { 2 - 8 } & $1000 \mathrm{~L} / 1 \mathrm{~m}^{3} / 1000 \mathrm{Wh}$ & & & ON & ON & & \\
\hline \multirow{3}{*}{$\begin{array}{l}\text { Periodo de } \\
\text { transmisión }\end{array}$} & $12 \mathrm{~h}$ & & & & & OFF & OFF \\
\cline { 2 - 8 } & $30 \mathrm{mn}$ & & & & & OFF & ON \\
\cline { 2 - 8 } & $15 \mathrm{mn}$ & & & & & ON & ON \\
\cline { 2 - 7 } & $10 \mathrm{mn}$ & & & & ON \\
\hline
\end{tabular}

Tabla 3.11. Posibilidades de configuración mediante los micro-interruptores.

En esta aplicación la configuración de los módulos se ha establecido con los siguientes parámetros en la Tabla 3.12.

\begin{tabular}{|c|c|c|c|}
\hline Parameter & Value & \multicolumn{2}{|c|}{ SW } \\
\hline Type & Water & SW1: OFF & SW2: OFF \\
\hline Pulse weight & $1 \mathrm{~L}$ & SW3: OFF & SW4: OFF \\
\hline Period & $30 \mathrm{~min}$ & SW5: OFF & SW6: ON \\
\hline
\end{tabular}

Tabla 3.12. Selección para esta aplicación de los micro-interruptores de configuración. 
La configuración por micro-interruptores debe ser establecida antes de activar los dispositivos. Una vez que el cable de fraude ha sido instalado y el dispositivo ha sido iniciado con el imán, la configuración no puede ser cambiada.

Después de realizar el conexionado del cableado y la configuración mediante los microinterruptores, el dispositivo ha de ser iniciado situando un imán en la posición indicada en la Figura 3.64 durante un mínimo de 6 segundos. Al iniciarse transmitirá una trama.

Una vez iniciado, no puede apagarse. Sin embargo, si se sitúa el imán una segunda vez, el dispositivo conmuta a modo configuración durante 4 minutos y luego vuelve al modo operativo.

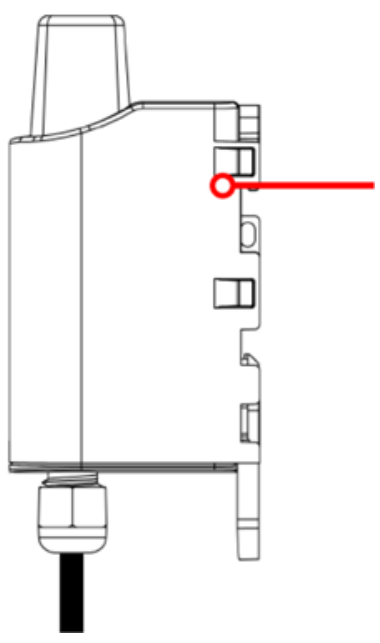

Figura 3.64. Posición del imán para el inicio de operación.

\subsubsection{Registro en el backend de Sigfox}

Los datos relacionados con el dispositivo que son necesarios para su registro en el Backend de Sigfox son:

- ID

- PAC

- Certificado del fabricante

Para incluir en un contrato ya existente a un nuevo dispositivo, los pasos a realizar son:

- Group $\rightarrow$ New

- Device Type $\rightarrow$ New

- Seleccionar grupo anteriormente creado

- Seleccionar contrato

- Device $\rightarrow$ New

- Seleccionar grupo anteriormente creado

- Seleccionar tipo anteriormente creado

Para configurar una visualización personalizada de las tramas enviadas por el dispositivo:

Device Type $\rightarrow$ (Dispositivo) $\rightarrow$ Payload display

Payload parsing: Custom gramar 
Custom configuration: Frame-Type: : uint: 8 Frame-Index:1:uint: $4:: 7$ Error:1:uint:4::3 Pulse-Type: uint:8 Counter::uint:32:littleendian

Los mensajes recibidos y mostrados en el Backend son del tipo mostrado en la Figura 3.65.

\begin{tabular}{|c|c|c|c|c|}
\hline Time & Data / Decoding & Location & Link quality & Callbacks \\
\hline 2018-05-29 19:10:47 & $02 f 006760 c 00000000000000$ & $\theta$ & IIIll & () \\
\hline $2018-05-29$ 18:41:59 & $02 \mathrm{e} 006540 \mathrm{~b} 00000000000000$ & $\theta$ & IIIll & () \\
\hline 2018-05-29 18:13:04 & $02 d 006320 a 00000000000000$ & $\theta$ & III & (1) \\
\hline $2018-05-29$ 17:44:10 & $02 c 006100900000000000000$ & $\phi$ & ıIlll & (4) \\
\hline 2018-05-29 17:15:21 & 02b006ee 0700000000000000 & $\theta$ & ıIlll & () \\
\hline 2018-05-29 16:46:32 & $02 \mathrm{a} 006 \mathrm{~d} 60600000000000000$ & $\psi$ & IIIll & (1) \\
\hline $2018-05-29$ 16:17:38 & $029006 \mathrm{~b} 40500000000000000$ & $\theta$ & IIIII & () \\
\hline $2018-05-29$ 15:48:50 & 028006920400000000000000 & $\theta$ & III & (1) \\
\hline 2018-05-29 15:19:57 & 027006700300000000000000 & $\theta$ & IIIll & (1) \\
\hline $2018-05-2914: 51: 03$ & $0260064 \mathrm{e} 0200000000000000$ & $\theta$ & IIIl & (1) \\
\hline 2018-05-29 14:22:11 & $0250062 c 0100000000000000$ & $\theta$ & IIIII & (1) \\
\hline $2018-05-29$ 13:53:20 & $0240060 a 0000000000000000$ & $\theta$ & ıIIII & ( \\
\hline 2018-05-29 13:53:02 & 0530000000000000 & $\theta$ & IIIll & () \\
\hline $2018-05-29$ 13:52:50 & $042000000 a 000000$ & $\theta$ & IIIll & (1) \\
\hline 2018-05-29 13:52:34 & 03100200000103030001 & $\theta$ & ıllll & ( \\
\hline
\end{tabular}

Figura 3.65. Visualización de mensajes en el backend de Sigfox.

\subsubsection{Replicador de pulsos}

Un contador preparado para tele-lectura dispone de una salida de impulsos con el fin de hacer llegar la información de consumo a un registrador o a un dispositivo de telecomunicaciones. Si se necesita utilizar un mismo contador con más de un dispositivo de manera simultánea, se hace necesario un replicador de pulsos. Esta es la finalidad del dispositivo aquí descrito.

\subsubsection{Descripción}

El dispositivo cuenta con una entrada para un contador con salida de impulsos. La salida del contador ha de ser libre de potencial. Así mismo, se encuentran disponibles 7 salidas libres de 
potencial mediante relés de estado sólido que replican los pulsos presentes a la entrada. No es necesario alimentar el dispositivo pues dispone de una batería interna de Litio. El formato de la carcasa permite su anclaje en carril DIN y puede verse en la Figura 3.66.
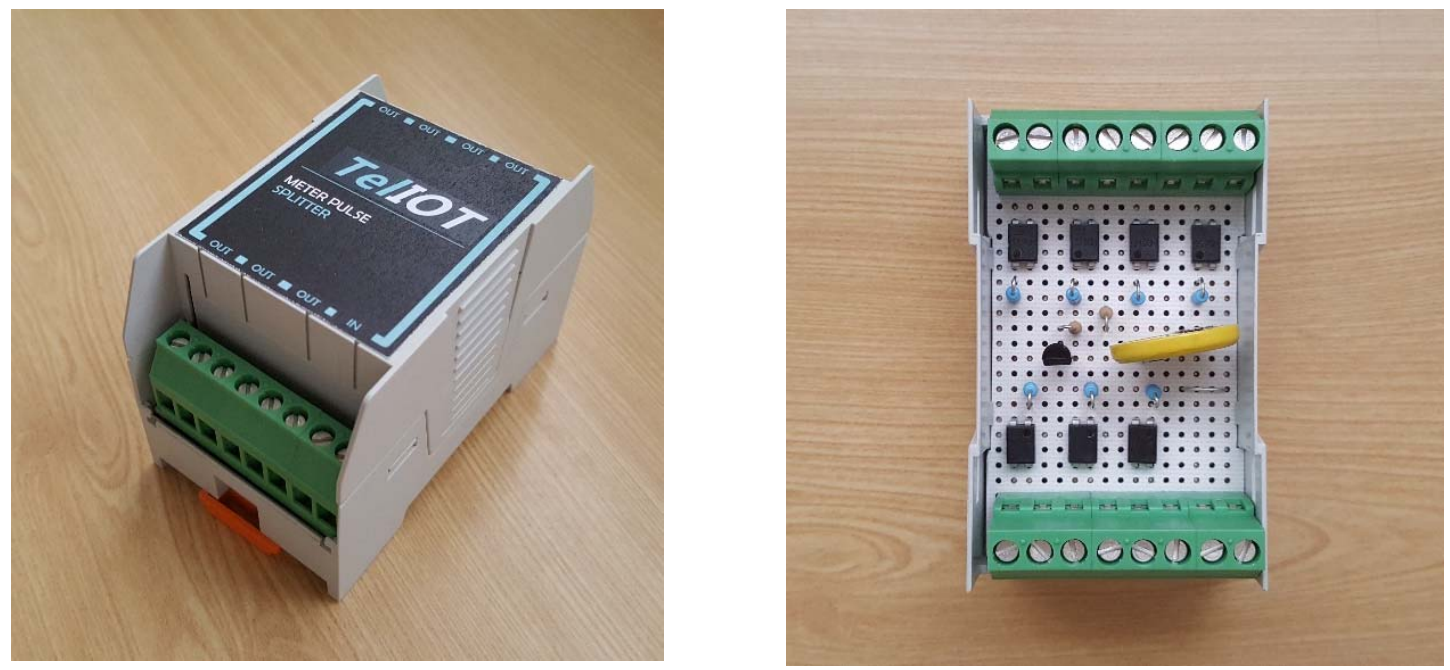

Figura 3.66. Vistas exterior e interior del dispositivo.

\subsubsection{Desarrollo hardware}

El esquemático electrónico puede consultarse en la Figura 3.67.

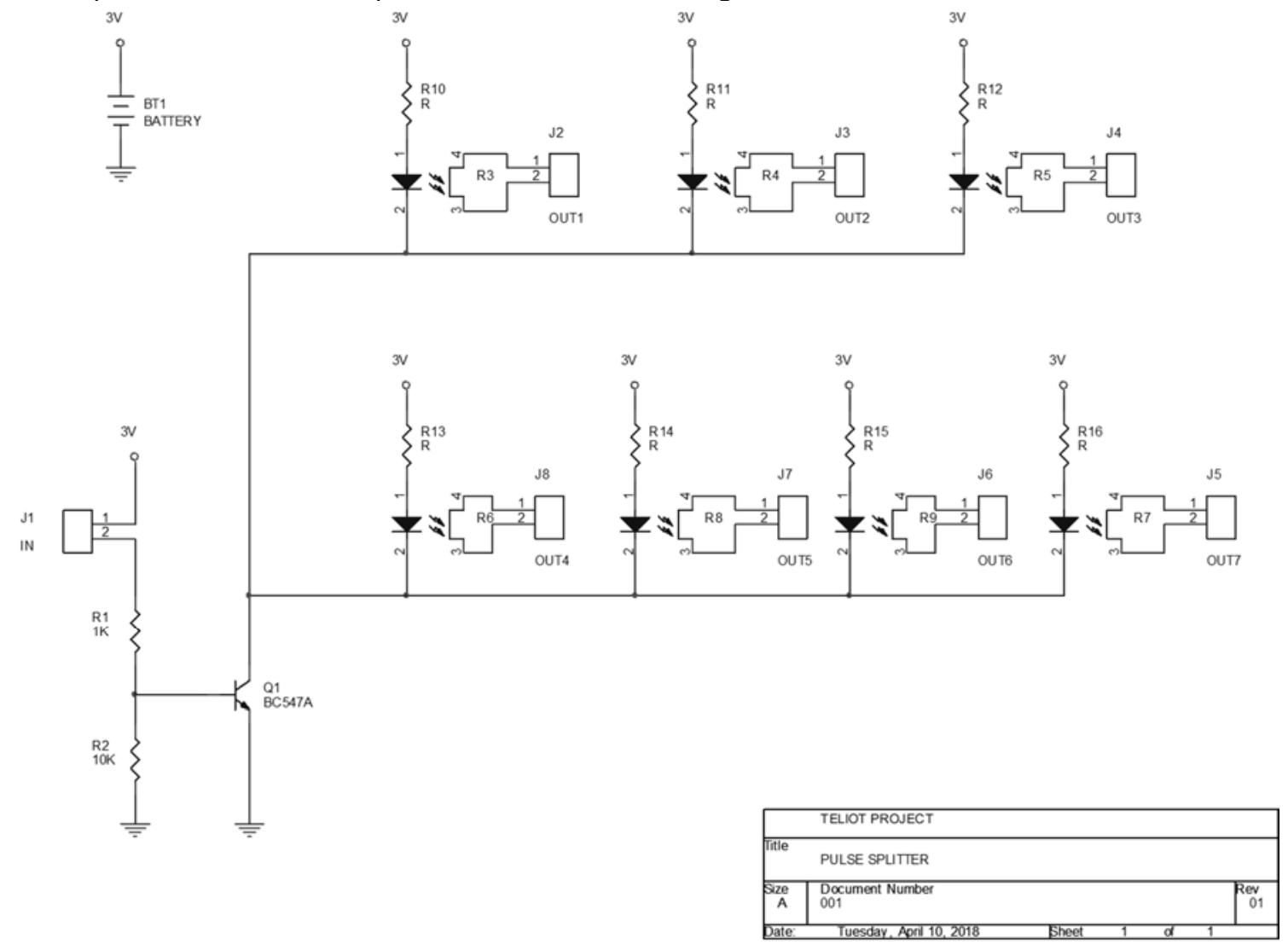

Figura 3.67. Esquemático eléctrico. 
A partir de los datos proporcionados por el fabricante de los relés de estado sólido de caída de tensión y corriente de excitación, se calcula la resistencia limitadora de la corriente de activación que se ha considerado de $7.8 \mathrm{~mA}$ como un valor medio entre los límites de $5 \mathrm{~mA}$ y $10 \mathrm{~mA}$ :

$$
\left.\begin{array}{c}
\mathrm{V}_{\mathrm{F}}=1.32 \mathrm{~V} \\
\mathrm{I}_{\mathrm{F}}=5-10 \mathrm{~mA}
\end{array}\right\} \rightarrow \mathrm{R}_{\mathrm{F}}=\frac{3-1.32}{7.8 \mathrm{~mA}}=220 \Omega
$$

La alimentación del dispositivo proviene de una batería interna de Litio de tipo botón, dado que en ausencia de pulso de entrada el consumo es despreciable y cuando este se produce, al ser el consumo también reducido, proporciona una autonomía en el rango a la ofrecida por los dispositivos transmisores. Las mediciones de diferentes parámetros con objeto de su verificación pueden verse en la Tabla 3.13.

\begin{tabular}{|c|c|c|c|}
\hline PARÁMETRO & CONDICIÓN & MEDIDA & UNIDAD \\
\hline I activacion 1 SCR & & 8.1 & $\mathrm{~mA}$ \\
\hline I activacion 8 SCR & & 62.7 & $\mathrm{~mA}$ \\
\hline V entrada SCR & & 1.17 & $\mathrm{~V}$ \\
\hline I batería & Reposo & 0.0 & $\mathrm{uA}$ \\
\hline I entrada contador & Pulso & 1.6 & $\mathrm{~mA}$ \\
\hline V batería & Reposo & 3 & $\mathrm{~V}$ \\
\hline V batería & Pulso & 2.4 & $\mathrm{~V}$ \\
\hline I batería 8 SCR & Vbat $=2.4$ & 43 & $\mathrm{~mA}$ \\
\hline
\end{tabular}

Tabla 3.13. Valores medidos de los diferentes parámetros eléctricos.

El conexionado eléctrico del replicador de pulsos a los otros elementos de la consola de test, es el descrito a continuación y mostrado en la Figura 3.68.

Entradas:

1. Generador de pulsos Itron Cyble Sensor, 2 conductores (sin polaridad)

Salidas:

1. Adeunis Sigfox Pulse (sin polaridad)

2. Adeunis LoRaWAN Pulse (sin polaridad)

3. Adeunis W-MBus (sin polaridad)

4. Teliot Wasmote Sigfox/LoRaWAN/LoRa (sin polaridad)

5. Teliot Pycom Sigfox (sin polaridad)

6. Teliot Pycom LoraWAN (sin polaridad) 


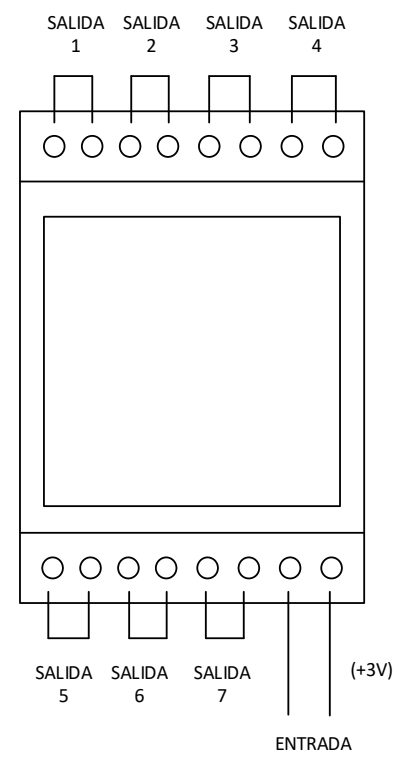

Figura 3.68. Diagrama de conexionado.

\subsubsection{Emulador de contador}

Un contador preparado para tele-lectura dispone de una salida de impulsos con el fin de hacer llegar la información de consumo a un registrador o a un dispositivo de telecomunicaciones. En un entorno de pruebas se hace necesario el disponer de un emulador de contador capaz de generar la misma salida que este con el fin de verificar el correcto funcionamiento de otros dispositivos.

\subsubsection{Descripción}

El emulador de contador presenta una interface de usuario mediante pantalla TFT táctil, a través de la cual es posible comprobar el estado de operación y modificar la configuración. A través de una salida libre de potencial se generan impulsos similares a los que el interface de lectura del contador produciría a diferentes caudales. Se pretende emular un contador modelo Flodis de Itron con un caudal nominal de $1.5 \mathrm{~m} 3 / \mathrm{h}$, un mínimo de $15 \mathrm{~L} / \mathrm{h}$ y un máximo de $3 \mathrm{~m} 3 / \mathrm{h}$, dotado de un interface de lectura con una constante de proporcionalidad $\mathrm{K}=1$.

En la Figura 3.69 pueden verse unas imágenes de la pantalla y del conjunto. 

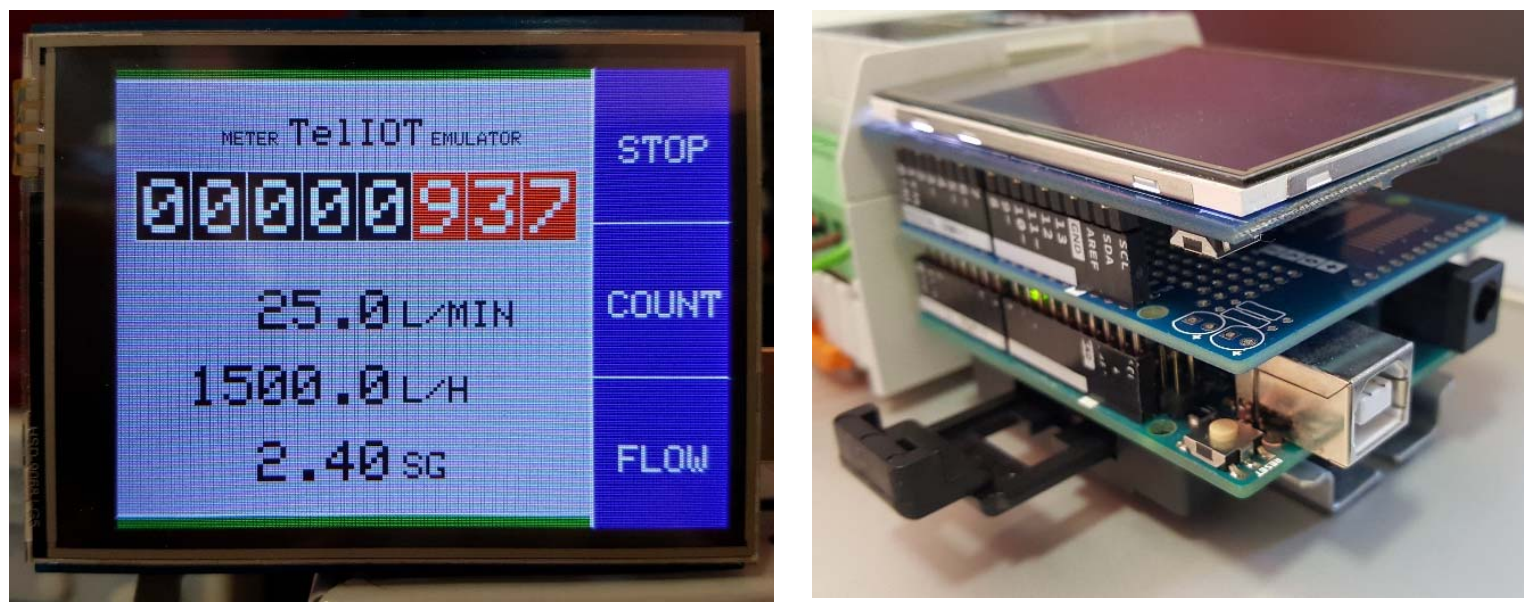

Figura 3.69. Interface de usuario y vista de conjunto.

Mediante los pulsadores en la parte derecha son posibles las siguientes funciones:

- START/STOP: Iniciar o detener la operación

- COUNT: Reiniciar el contador

- FLOW: Configurar el caudal

En la zona principal es posible visualizar:

- Valor actual de consumo como se presentaría en un contador. Las unidades son m3 en la última cifra de fondo negro y litros en la última de fondo rojo.

- Caudal emulado configurado en diferentes unidades: $1 / \mathrm{min}$, I/hora y segundos entre impulsos.

- Estado de operación en las zonas superior e inferior. Verde indica actividad y rojo que no se genera ninguna salida.

\subsubsection{Desarrollo hardware}

Como elemento controlador se ha utilizado una unidad Arduino Uno. A ella y utilizando los conectores de expansión, se ha acoplado una placa de prototipado donde se ubican los componentes necesarios para proporcionar una salida libre de potencial. A su vez, a esta placa se acopla la pantalla TFT, quedando el conjunto formado por tres placas apiladas pero sin sobrepasar las dimensiones de ancho y largo de Arduino Uno y sin necesitar cableado adicional entre ellas. Se incluye además un elemento de acople para carril DIN.

La generación de pulsos se lleva a cabo a través de una salida digital que a su vez excita un relé de estado sólido. Este relé permite controlar un circuito externo mediante la apertura o cierre de su salida libre de potencial. Al tratarse de un relé de estado sólido sin partes mecánicas móviles, su durabilidad es mayor y permite unos tiempos de conmutación inferiores.

La pantalla TFT de 2.8" y 240x320 pixels de resolución, se comunica con el procesador a través del bus SPI, tanto para la visualización como para el interface de entrada táctil resistivo. Recibe alimentación de $5 \mathrm{~V}$ desde la placa principal.

La alimentación del conjunto puede realizarse a través del conector USB, que también sirve para actualizar el firmware, o a través de una regleta dispuesta para tal fin en la placa de expansión. 
En ambos casos ha de ser una tensión regulada de $5 \mathrm{~V}$ y al menos $500 \mathrm{~mA}$ de corriente. Otra opción es una tensión de entre 7-12 V por el conector circular específico de alimentación.

En la Figura 3.70 se encuentra representado el esquemático eléctrico del dispositivo.

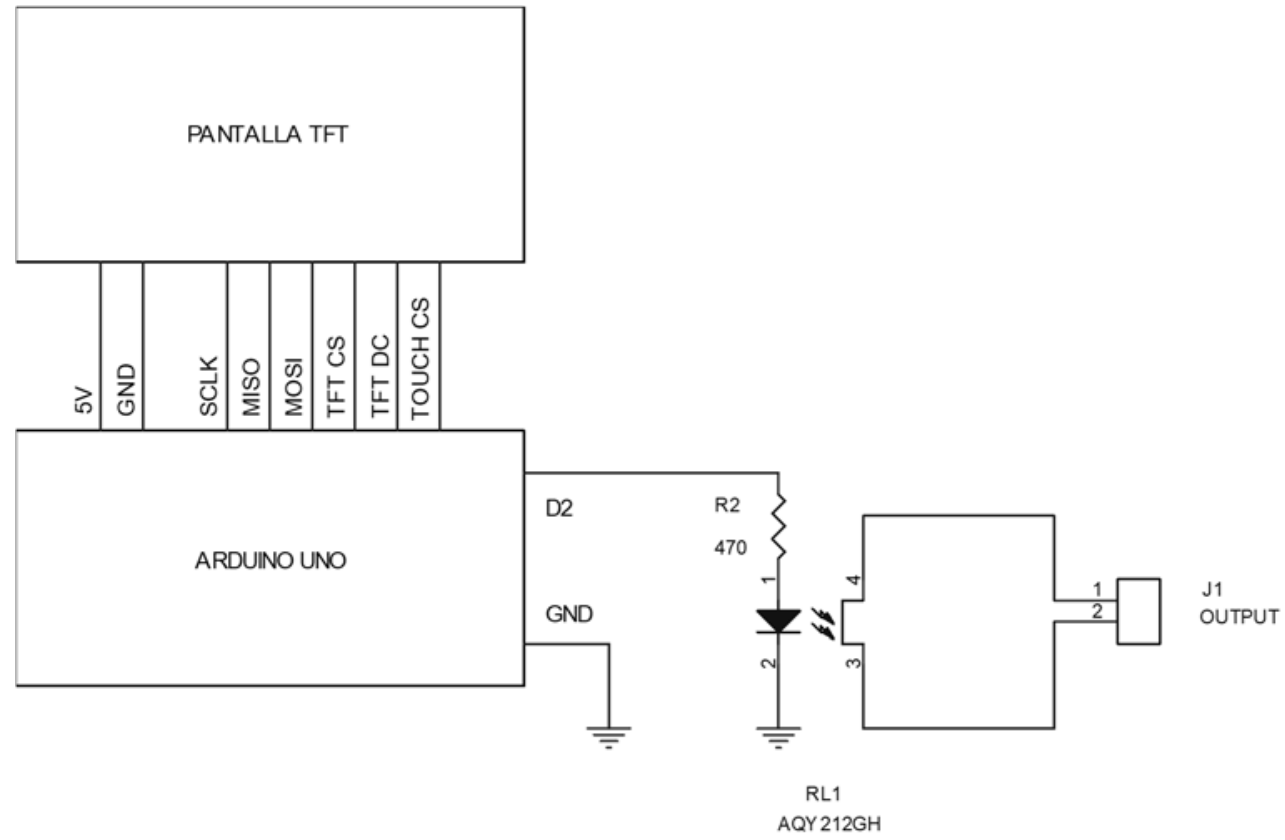

Figura 3.70. Esquemático eléctrico.

El cálculo eléctrico de la resistencia limitadora de corriente para la excitación del relé de estado sólido, es el siguiente acorde a la tensión directa Vf y corriente directa If proporcionadas por el fabricante:

$$
\left.\begin{array}{c}
\mathrm{V}_{\mathrm{F}}=1.32 \mathrm{~V} \\
\mathrm{I}_{\mathrm{F}}=5-10 \mathrm{~mA}
\end{array}\right\} \rightarrow \mathrm{R}_{\mathrm{F}}=\frac{5-1.32}{7.8 \mathrm{~mA}}=471 \Omega
$$

El rango de la señal generada puede verse en la Tabla 3.14, la señal generada por el módulo de control para la excitación del relé de salida se encuentra en la Figura 3.71 y la forma de onda de la salida generada en la carga, está en la Figura 3.72.

\begin{tabular}{|c|c|c|c|c|}
\cline { 2 - 5 } \multicolumn{1}{c|}{} & $\mathrm{m}^{3} / \mathrm{h}$ & $\mathrm{I} / \mathrm{h}$ & $\mathrm{I} / \mathrm{min}$ & $\mathrm{s} / \mathrm{pulso}(\mathrm{K}=\mathbf{1})$ \\
\hline Mínimo & 0,015 & 15 & 0,25 & 240 \\
\hline Nominal & 1,5 & 1500 & 25 & 2,4 \\
\hline Máximo & 3 & 3000 & 50 & 1,2 \\
\hline
\end{tabular}

Tabla 3.14. Rango de salida 


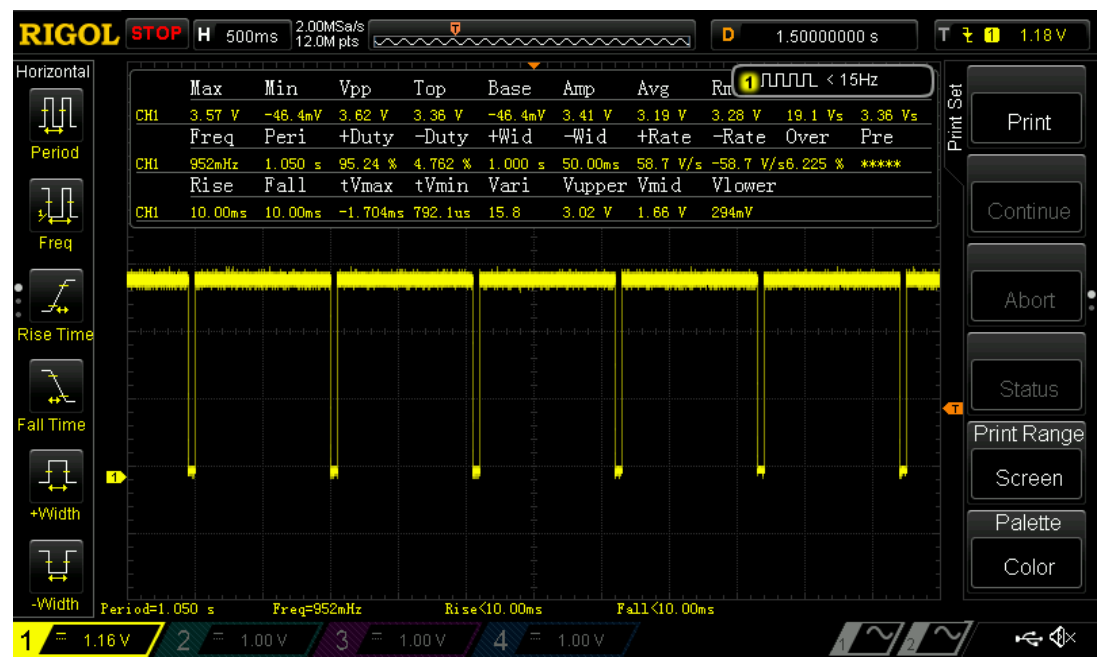

Figura 3.71. Tensión en extremos del relé de salida.

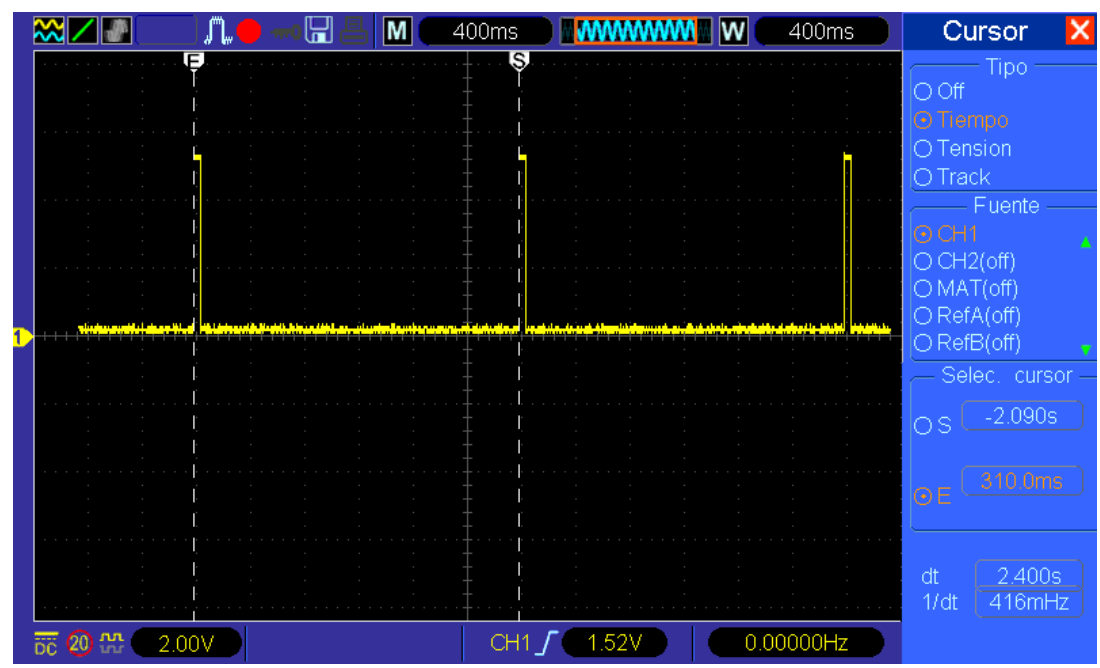

Figura 3.72. Pulsos generados en la carga.

\subsubsection{Desarrollo software}

La operación del software embebido es la siguiente. Tras conectar la alimentación e iniciarse la ejecución del software embebido, se llevan a cabo una serie de tareas iniciales tales como la configuración en modo salida del pin digital que generará la forma de onda y la configuración e inicialización de la pantalla y su panel táctil.

Pasada la fase de inicialización, se llevan a cabo una serie de tareas de manera continua hasta que se retira la alimentación. Estas son, la lectura del panel táctil y obtención de coordenadas en caso de que se haya efectuado una pulsación. La ejecución de las correspondientes acciones en caso de que se haya pulsado alguno de los botones virtuales. Y la generación en la salida de la correspondiente forma de onda en caso de que esta se haya habilitado.

Son 3 los botones virtuales que se encuentran en la parte derecha de la pantalla. El etiquetado como "RUN" activa o desactiva la generación de la onda en la salida. El estado se indica con unas barras de color verde (on) y rojo (off) en la parte superior e inferior de la zona principal de la 
pantalla. El botón "COUNT" permite inicializar el contador a un valor de cero. Por último, el botón "FLOW" permite ir variando con cada pulsación el caudal de agua que circularía por el contador emulado. Los valores varían de $0.10 \mathrm{~L} / \mathrm{min}(6 \mathrm{~L} / \mathrm{h})$, lo que supone un pulso cada $600 \mathrm{sg}$, a 50 $\mathrm{L} / \mathrm{min}(3000 \mathrm{~L} / \mathrm{h})$, lo que supone un pulso cada 1.2 segundos. Los valores configurados son mostrados en la zona principal de la pantalla, así como el conteo actual.

En la Figura 3.73 se encuentra indicado el flujograma del software embebido.
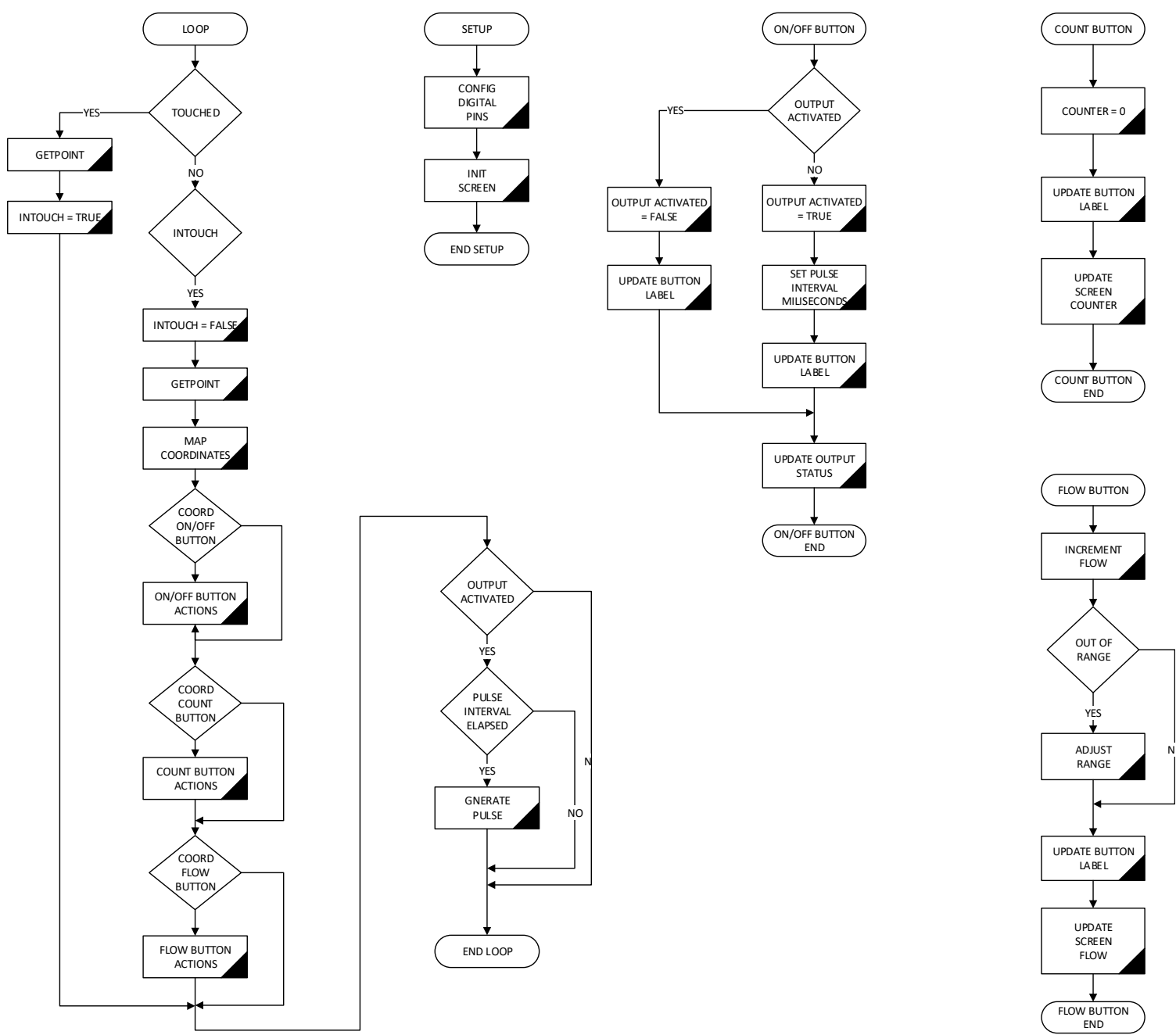

Figura 3.73. Diagrama de flujo.

\subsubsection{Maqueta de pruebas en laboratorio}

Para llevar a cabo las primeras verificaciones en laboratorio se ha utilizado una maqueta consistente en un circuito cerrado de agua impulsada por una electrobomba y con un contador instalado del mismo a los utilizados en las viviendas de abonados.

La maqueta consta de los siguientes elementos:

- Depósito de agua con salida de drenaje 
- Electrobomba

- Tuberías conformando un circuito cerrado con salida y llegada al depósito

- Manómetro

- Contador de consumo en circuito principal

- Contador de consumo en circuito secundario simulando línea de abonado

- Llave de paso

En la Figura 3.74 puede verse una imagen de la maqueta.

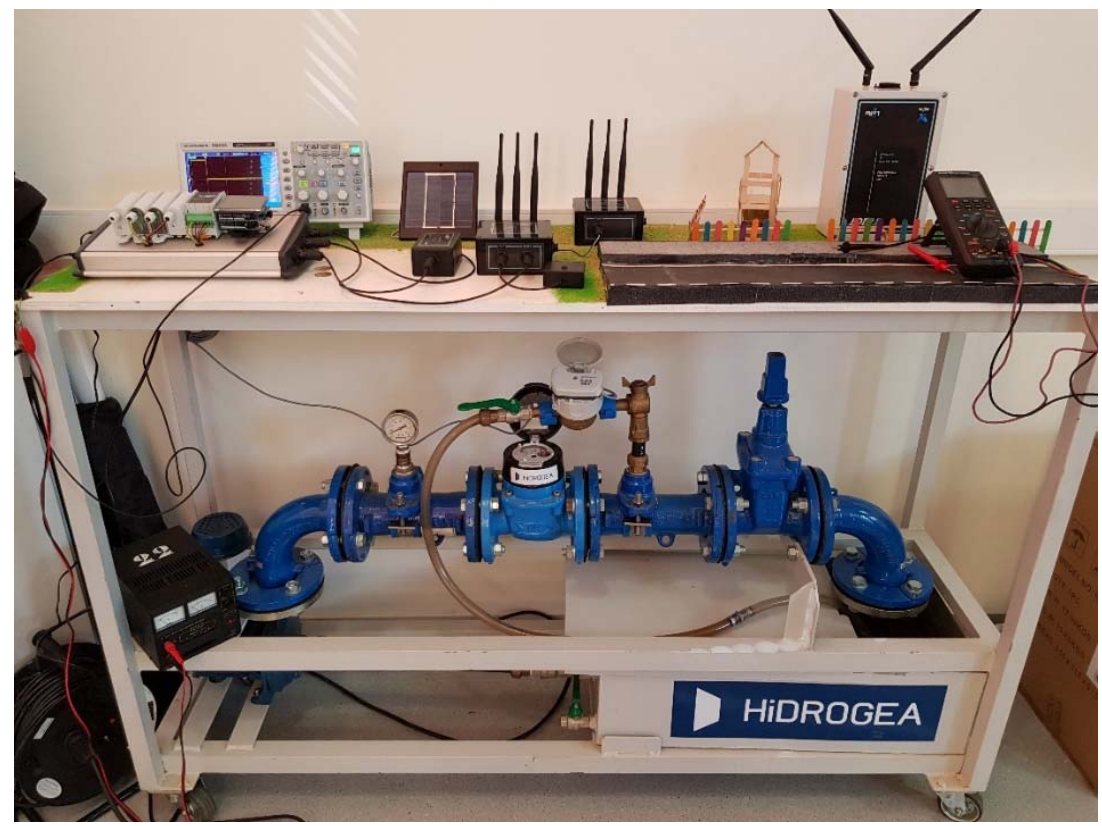

Figura 3.74. Maqueta de pruebas en laboratorio.

Se ha podido verificar el funcionamiento del dispositivo de captura de información (Itron Cyble Sensor) y visualizar su salida acoplándolo al contador de abonado de la maqueta. Así mismo, poniendo en funcionamiento la electrobomba y creando un flujo de agua que simula un consumo en una vivienda, se ha podido verificar que los dispositivos de tele-lectura diseñados, eran capaces de adquirir la información del contador y enviarla correctamente a través de las redes loT. En la Figura 3.75 pueden verse los dispositivos operando con la maqueta.

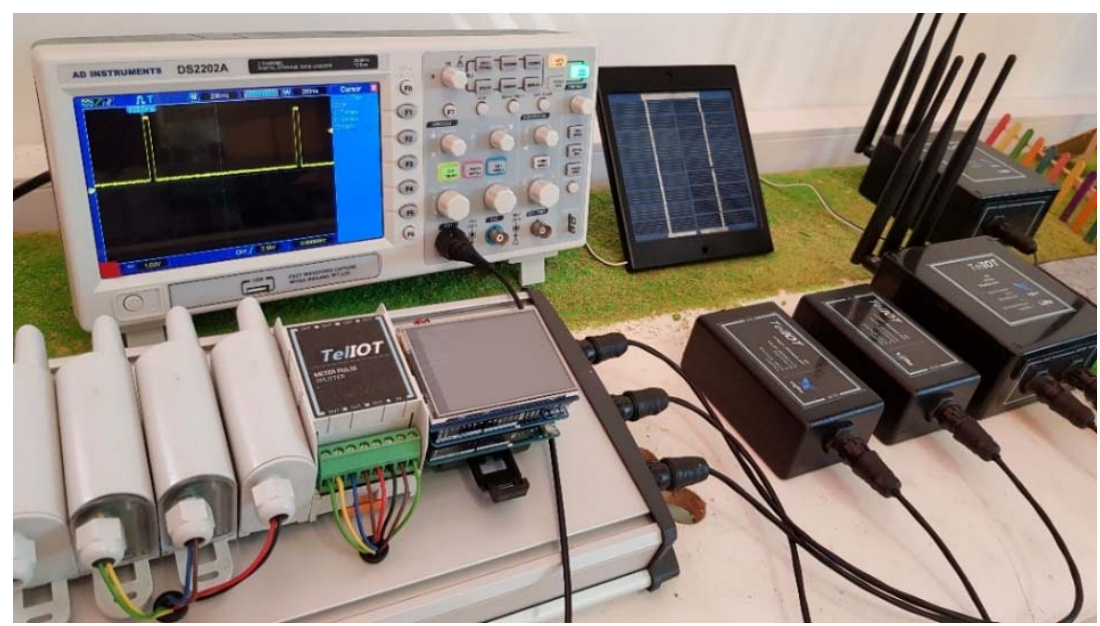

Figura 3.75. Dispositivos diseñados operando con la maqueta. 


\subsection{Conclusiones}

Se ha diseñado una arquitectura de red mixta y flexible, capaz de combinar diferentes soluciones de comunicación loT, que permite la conexión de objetos loT sin la restricción de la tecnología empleada y capaz de adaptarse durante el despliegue a las necesidades de cada aplicación. Se ofrece además una solución para ubicaciones conflictivas en zonas de sombra de cobertura.

Además de habilitar la conexión de los dispositivos comercialmente disponibles en la actualidad para tele-lectura, se ha identificado como una prestación adicional innovadora y que crea un valor añadido a los nodos finales, la capacidad de actuar de manera remota sobre un elemento externo tal como una electroválvula. De esta manera, la compañía suministradora del suministro de agua, sería capaz no sólo de tener información de consumo, sino de controlar el servicio al abonado final.

Se han desarrollado y construido varios dispositivos hardware propios para su integración en la arquitectura propuesta con objeto de satisfacer las necesidades que se han ido presentando en el despliegue de la red y que no estaban cubiertas por productos ya existentes. La intención también ha sido de diseñar empleando hardware y software abiertos, que proporcionasen libertad para implementar la funcionalidad requerida y facilitasen posibles ampliaciones futuras y soluciones propias sin las limitaciones de los productos comerciales cerrados.

Los dispositivos propios son los siguientes. El elemento principal es un dispositivo de adquisición de datos de contador de agua residencial capaz de controlar un elemento externo como una electroválvula y con conectividad múltiple, tanto a redes LoRaWAN, como a la red Sigfox directamente o mediante el gateway propio. Se han creado dos versiones de este dispositivo, la primera de ellas empleando el controlador Waspmote. Como una optimización tanto en tamaño físico como en costes, se ha diseñado una nueva versión basada en el controlador PyCom pero contando con las mismas características de conectividad y control. El denominado gateway loT es un dispositivo intermedio que situado en una ubicación estratégica y haciendo uso de un enlace LoRa, soluciona la operación del dispositivo contador en zonas de sombra de cobertura Sigfox. Por último, se ha construido una estación base LoRaWAN basada en hardware y software abiertos y con coste inferior al de soluciones comerciales que ofrecen la misma funcionalidad.

De manera adicional y como asistencia en el desarrollo de este trabajo, se ha creado un dispositivo propio para facilitar el estudio de cobertura, así como una consola de pruebas con emulador de contador y repartidor de su señal para la fase de validación en laboratorio. 
CAPÍTULO 4

EVALUACIÓN DE LA SOLUCIÓN PROPUESTA 



\subsection{Introducción}

Previo a la implantación de un caso de real de estudio, se hace necesario verificar que una conectividad específica para loT utilizando tecnologías LPWAN es aplicable. Para ello, se procederá a estudiar y probar la tecnología en entorno urbano y en situaciones desfavorables. Se verificará la cobertura ofrecida por el operador Sigfox a nivel de calle y se comprobará la cobertura proporcionada por una estación base LoRaWAN de propia instalación.

Con objeto de evaluar tanto los dispositivos comerciales disponibles como los desarrollados a propósito de este trabajo, se llevará a cabo una validación en el entorno de laboratorio montado al efecto.

Una vez que se ha confirmado en laboratorio el correcto funcionamiento de los dispositivos, se han puesto a prueba todas sus capacidades, y se han verificado los datos transmitidos, se ha pasado a desarrollar un caso de estudio en condiciones reales de funcionamiento. Ha consistido en la puesta en marcha de dispositivos en múltiples ubicaciones del entorno urbano con diferentes tipos de conectividad, para posteriormente evaluar los resultados obtenidos.

\subsection{Detección de la actividad del contador}

Se ha experimentado la posibilidad de detectar por medios propios el movimiento de la aguja del contador y generar una señal eléctrica utilizable por un transmisor de consumo.

Para las pruebas se ha utilizado el contador Flodis de Itron. La aguja tiene acoplado un elemento metálico, pero dado que este no está imantado, no es posible detectar su paso mediante relés de tipo reed o sensores de efecto hall. La superficie metálica es en torno a $5 \mathrm{~mm}$ y la aguja se encuentra a una profundidad de $4 \mathrm{~mm}$ respecto a la superficie. Para detectarla se ha seleccionado el sensor inductivo E2B-S08KN04-WP-C1 2M OMS del fabricante Omron por las características mostradas en la Tabla 4.1 y que se consideran idoneas para el fin perseguido.

\begin{tabular}{|c|c|}
\hline CARACTERÍSTICA & VALOR \\
\hline Tecnología & Inductivo \\
\hline Distancia detección & $4 \mathrm{~mm}$ \\
\hline Superficie detección & $6.6 \mathrm{~mm}$ \\
\hline Objeto detectado & $12 \times 12 \times 1$ \\
\hline Rosca & $\mathrm{M} 8$ \\
\hline Cuerpo & Cilíndrico de metal \\
\hline Alimentación & $12-24 \mathrm{Vdc}$ \\
\hline Carga máxima & $200 \mathrm{~mA}$ \\
\hline Salida & $\mathrm{NPN}$ \\
\hline Protección & $\mathrm{IP67}$ \\
\hline
\end{tabular}

Tabla 4.1. Especificaciones técnicas del sensor E2B-S08KN04-WP-C1. 
La alimentación utilizada ha sido de 12 Vdc y debido a que la salida es con transistor de tipo NPN, se genera un nivel lógico a cada paso de la aguja. La correcta conexión de alimentación y carga se muestra en la Figura 4.1.

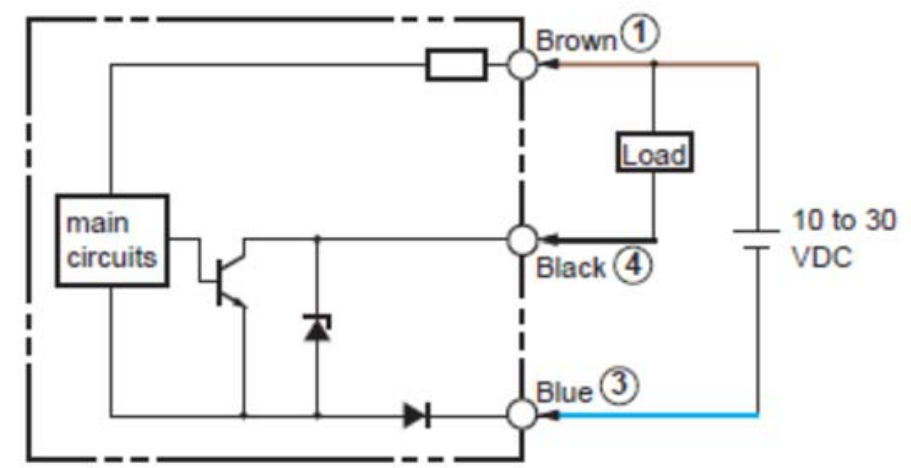

Figura 4.1. Conexión del sensor.

Se ha procedido a situar el sensor de forma vertical sobre la superficie del contador de manera que se produzca detección de la aguja al pasar esta por una zona concreta de la esfera tal y como puede verse en la Figura 4.2. A pesar de que la superficie metálica adjunta a la aguja del contador es inferior a los requisitos mínimos de detección del fabricante del sensor, se ha podido comprobar que la detección de esta se produce correctamente.
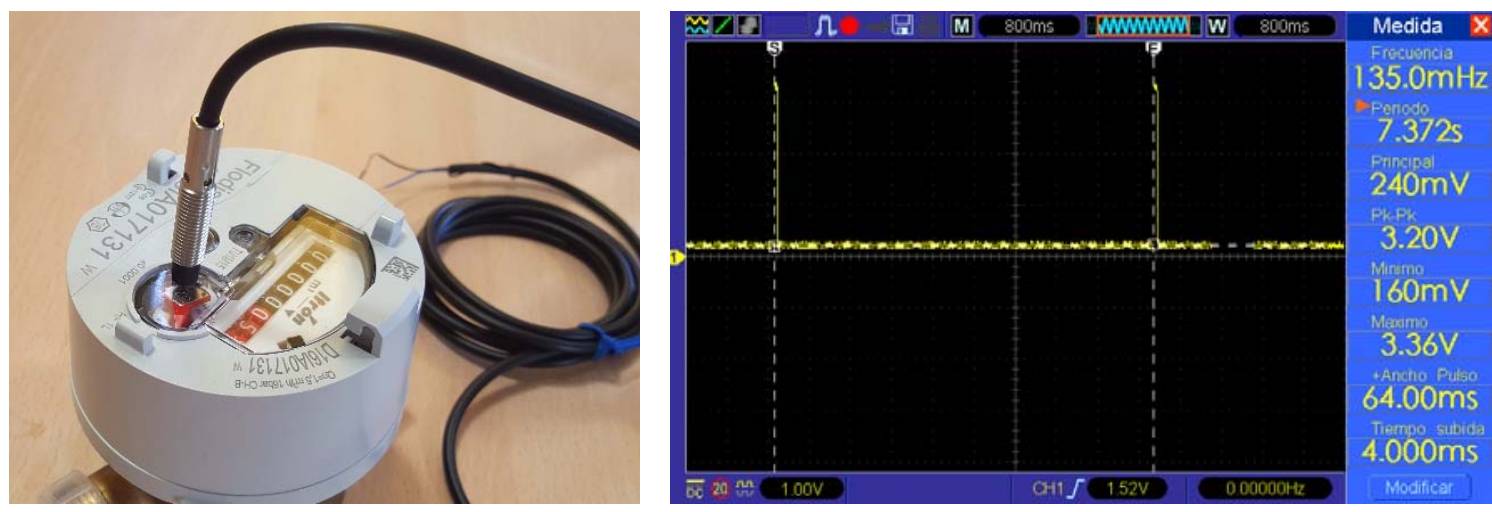

Figura 4.2. Detección de giro de aguja con sensor inductivo.

Al respecto del empleo de un sensor industrial para la lectura de consumo, a pesar de que los resultados fueron satisfactorios, en aplicaciones prácticas, debido a la longitud del sensor y su método de sujeción roscado, sería necesario diseñar y construir una carcasa adaptadora para su firme fijación al contador. Al tratarse de un solo sensor, no es posible discernir el sentido de giro de la aguja, para ello serían necesarios al menos dos de ellos. Además, la tensión de alimentación mínima de 12 Vdc y el consumo de corriente continuo de $10 \mathrm{~mA}$, requeriría alimentación permanente 0 una batería de gran capacidad capaz de proporcionar una autonomía aceptable. En caso de que el dispositivo al que se conectase no aceptase la salida NPN del sensor por requerir una salida libre de potencial o de lógica positiva, sería necesario añadir en el interior de la carcasa circuitería electrónica adaptadora de niveles. Por todo ello, se concluye el uso de un conjunto sensor y generador de impulsos comercial como el proporcionado por el fabricante del contador ya diseñado para acoplarse a este y con fiabilidad homologada, centrándonos en la transmisión de información con tecnologías específicas de loT. 


\subsection{Estudio de cobertura de redes LPWAN}

Se pretende verificar que puede haber cobertura de servicio en el núcleo urbano de una ciudad como Cartagena y es por tanto factible dotar de conectividad IoT a dispositivos que se ubiquen entre edificios, sin línea visual en el enlace radio, que incluyan una antena integrada, con potencia de salida de $25 \mathrm{~mW}$ en la banda de $868 \mathrm{MHz}$, modulación Sigfox o LoRa y con el ruido radioeléctrico y perturbaciones propias de un área densamente poblada.

Para esta fase se han utilizado los medidores de campo del fabricante Adeunis para la generación de una señal acorde a las frecuencias, modulaciones y protocolos necesarios para establecer una comunicación tanto Sigfox como LoRaWAN. Se consideran aplicables a los dispositivos de telemedida y telecontrol los resultados obtenidos con el demostrador de campo ya que, la sensibilidad del receptor es la misma, $-130 \mathrm{dBm}$ en el caso de Sigfox y $-140 \mathrm{dBm}$ en el caso de LoRa; la potencia de transmisión es la misma, $14 \mathrm{dBm}$; y aunque la antena del medidor de campo es externa, corresponde a $1 / 4$ de longitud onda y $0 \mathrm{dBi}$.

Se ha tomado en consideración el RSSI y SNR presentados en la pantalla del equipo que corresponden respectivamente a la intensidad de señal y a la relación señal-ruido con las que se ha recibido la transmisión de bajada procedente de una estación base de la red. Sin embargo, como en nuestra aplicación el flujo de información relevante son los datos de consumo del usuario que van en el enlace ascendente, se han tenido en mayor consideración los niveles de señal con que la red recibe al dispositivo móvil.

Las pruebas se han efectuado dispositivo en mano caminando en el entorno urbano de la ciudad de Cartagena. Se han tratado de buscar localizaciones difíciles para la propagación de una señal de radiofrecuencia en la banda de $868 \mathrm{MHz}$, en calles con edificios altos, sin línea visual con las estaciones base, que sería el caso ideal para la propagación de señal en esa banda y por tanto máximo alcance. En determinados casos se han provocado casos difíciles como la prueba de enlace en plantas bajas interiores, cajas de contador en interior de pared con puerta metálica e incluso sótanos, ya que en el caso de aplicación real, los contadores de abonado están en localizaciones de este tipo.

\subsubsection{Estudio de cobertura Sigfox}

\subsubsection{Medidas en entorno urbano}

En la Figura 4.3 puede verse un mapa con marcadores posicionados en los puntos de la ciudad donde se ha efectuado una medida de cobertura a nivel de calle con el medidor de campo Adeunis ARF8121AA. El color está asociado al nivel de señal con que la red recibe al dispositivo, variando desde los tonos más claros de amarillo que corresponden a valores por encima de -80 $\mathrm{dBm}$, hasta los tonos más oscuros de violeta que corresponden a valores de entre -130 y -140 $\mathrm{dBm}$. En la Tabla 4.2 se encuentra toda la correspondencia de niveles. 


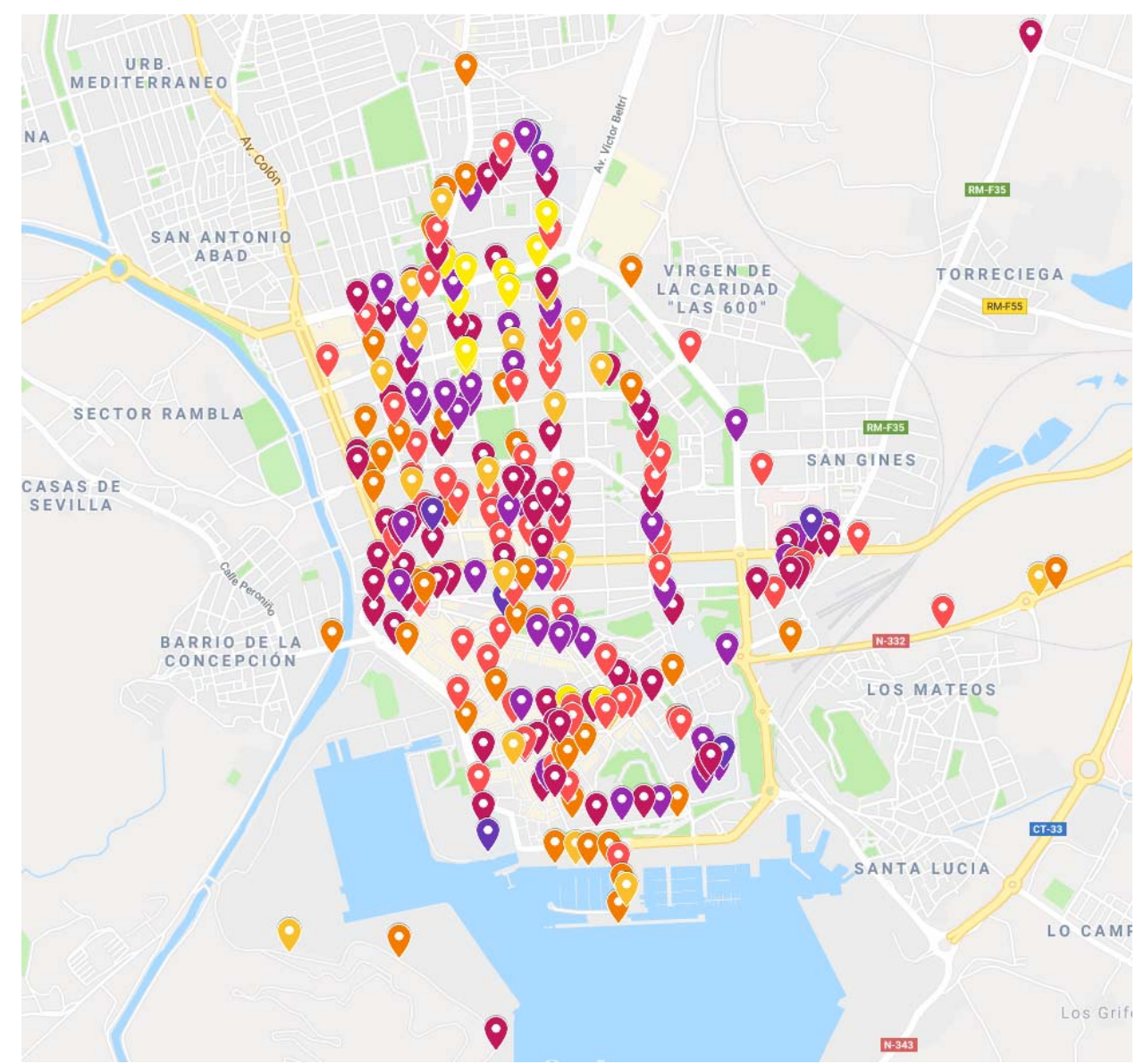

Figura 4.3. Medidas de cobertura Sigfox en Cartagena.

\begin{tabular}{|c|c|}
\hline RSSI (dBm) & COLOR \\
\hline$<-140$ & NEGRO \\
\hline$[-140,-130]$ & VIOLETA \\
\hline$[-130,-120]$ & ROSA \\
\hline$[-120,-110]$ & ROJO \\
\hline$[-110,-100]$ & BUTANO \\
\hline$[-100,-090]$ & NARANJA \\
\hline$[-90,-080]$ & AMBAR \\
\hline$>-80$ & AMARILLO \\
\hline
\end{tabular}

Tabla 4.2. Relación entre niveles de señal y color de marcadores.

En la zona del casco antiguo de la ciudad ha podido comprobarse que a una distancia de 250 metros de la estación base, bajo tierra, en el sótano de un edificio de 4 plantas, se puede establecer comunicación siendo recibido por la red y recibiendo la transmisión de bajada de esta. Los niveles de RSSI recibidos en la estación base son de entre -135 y -140 dBm.

Continuando con las pruebas de cobertura en interior, a una distancia de 400 metros de la estación base y en el interior del edificio de la Escuela de Ingeniería Industrial que, por su antigua función militar, dispone de muros de piedra de 1 metro de espesor, se ha conseguido establecer enlace con la red con los niveles mostrados en la Tabla 4.3. Se ha establecido incluso comunicación en algún punto localizado en el sótano del edificio. 


\begin{tabular}{|c|c|c|c|c|c|c|}
\hline ID & UBICACIÓN & HORA & $\begin{array}{c}\text { ID } \\
\text { BASE }\end{array}$ & $\begin{array}{l}\text { RSSI } \\
\text { BASE } \\
(\mathrm{dBm})\end{array}$ & $\begin{array}{c}\text { SNR } \\
\text { BASE } \\
(\mathrm{dBm})\end{array}$ & $\begin{array}{c}\text { RSSI } \\
\text { DEVICE } \\
\text { (dBm) }\end{array}$ \\
\hline 1 & Sótano Entrada Izquierda & 10:11 & OBAD & -134 & 11.80 & -108 \\
\hline 2 & Media Escalera Izquierda & 10:12 & OBAD & -129 & 11.34 & -115 \\
\hline 3 & Entrada izquierda & $10: 14$ & 1DDB & -126 & 12.77 & -86 \\
\hline 4 & Entrada principal & 10:15 & 1DD7 & -128 & 6.54 & -85 \\
\hline 5 & Media Escalera Derecha & 10:17 & OBAD & -129 & 13.68 & -107 \\
\hline 6 & Entrada derecha & 10:18 & 1DD7 & -121 & 13.43 & -101 \\
\hline 7 & Bajo Este Sur - PB3 & $10: 20$ & OBAD & -121 & 10.44 & -99 \\
\hline 8 & Bajo Este Norte & $10: 22$ & OBAD & -123 & 13.42 & -112 \\
\hline 9 & Bajo Norte Este - PB5 & $10: 23$ & OBAD & -128 & 14.73 & -110 \\
\hline 10 & Bajo Cafetería & $10: 25$ & OBAD & -135 & 13.78 & -118 \\
\hline 11 & Bajo Norte Oeste & $10: 27$ & $1 \mathrm{DD} 7$ & -127 & 14.48 & -114 \\
\hline 12 & Bajo Oeste Norte - Aula informática & $10: 29$ & OBAD & -133 & 15.85 & -118 \\
\hline 13 & $1^{\mathrm{a}}$ Oeste Norte - P1.8 & 10:31 & 1DD7 & -124 & 14.31 & -106 \\
\hline 14 & $1^{\mathrm{a}}$ Oeste Sur - P1.3 & 10:34 & 1DD7 & -115 & 9.88 & -88 \\
\hline 15 & $1^{\mathrm{a}}$ Este Sur - Electricidad & $10: 36$ & $1 \mathrm{DDB}$ & -133 & 7.04 & -109 \\
\hline 16 & $1^{\mathrm{a}}$ Este Norte - DTE & 10:39 & OBAD & -117 & 14.25 & -102 \\
\hline 17 & Sótano Entrada Derecha & 10:05 & - & - & - & - \\
\hline 18 & Sótano Fondo Derecha & 10:07 & - & - & - & - \\
\hline 19 & Sótano Fondo Centro & 10:09 & - & - & - & - \\
\hline 20 & Sótano Fondo Izquierda & $10: 10$ & - & - & - & - \\
\hline
\end{tabular}

Tabla 4.3. Resultados de pruebas de cobertura en interiores.

La Figura 4.4 muestra un listado parcial en el backend de las tramas recibidas por la red desde esta ubicación difícil. Puede apreciarse como el indicador de nivel de señal está en valores medios y bajos y la calidad de señal, indicada por el color, no corresponde al caso ideal pero aun así sigue recibiéndose la información.

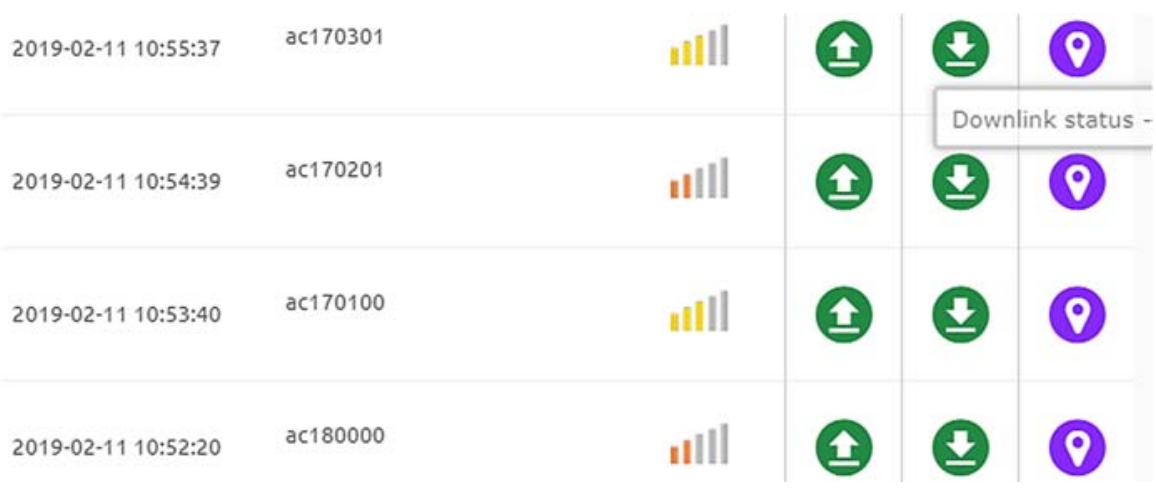

Figura 4.4. Listado de tramas recibidas desde un edificio fortificado.

Otra prueba destacable ha sido la efectuada en el garaje ubicado en un sótano en una barriada en las afueras del centro de la ciudad. La estación base que recibió la señal (1002) se encontraba ubicada a $1.56 \mathrm{~km}$ de distancia y la intensidad recibida osciló entre $-115 \mathrm{dBm}$ y -117 $\mathrm{dBm}$. En este caso no se consiguió recibir ninguna trama de bajada en el sentido red a dispositivo. Un listado parcial de tramas recibidas en el backend está en la Figura 4.5. 


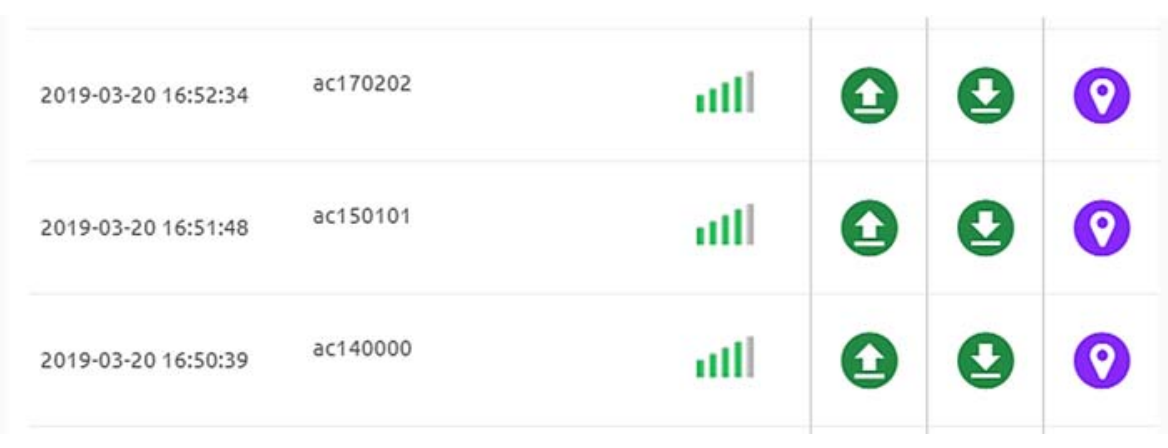

Figura 4.5. Listado de tramas recibidas desde un sótano.

En la mayoría de ubicaciones con posibilidad de enlace Sigfox, la cobertura de varias estaciones base se solapa para asegurar redundancia y ofrecer localización. Esto se ha podido apreciar en las pruebas ya que en algunas zonas, a pesar de tener más próxima cierta estación base, la señal ha sido recibida por otras estaciones de la red más lejanas. En algunas localizaciones como sótanos, no se ha llegado a recibir en el medidor de campo la señal de bajada procedente de la red, sin embargo esta ha sido capaz de recibir correctamente la señal transmitida por el dispositivo. Este hecho puede deberse además de por las mejores condiciones de recepción de las estaciones base, con antenas de mayor ganancia y amplificador de bajo ruido, al hecho de que el tipo de modulación es diferente en el enlace de subida y el de bajada, siendo más exigente en este último. En cualquier caso, al tratarse de una comunicación asíncrona que no requiere establecer una conexión bidireccional, los datos originados en el dispositivo final son recibidos y reenviados sin necesidad de completar el enlace descendente.

Como curiosidad cabe destacar que en la zona de la Muralla del Mar, al sur de la ciudad y lindando con el puerto, a pesar del obstáculo geológico natural que es el Cerro de La Concepción, la señal transmitida por el medidor de campo a nivel de calle fue recibida por la estación base ubicada en Los Barreros, en la zona norte de la ciudad a unos $3.5 \mathrm{~km}$ de distancia en línea recta y con todo el núcleo urbano entre ambas ubicaciones.

\subsubsection{Localización de estaciones base}

En base a la intensidad de la señal de la estación base que el medidor de campo ha recibido en diferentes localizaciones del entorno urbano y la intensidad de la señal con la que la red ha recibido al dispositivo, se han detectado zonas de la ciudad con unos valores de señal superiores a la media. Esto hace presuponer que una estación base de Sigfox se encuentra en las inmediaciones.

Como puede verse en la Figura 4.6, en la zona del casco urbano de la ciudad hay puntos en los que la red recibe al medidor de campo con intensidades superiores a $-80 \mathrm{dBm}$, representadas en amarillo, y puntos con intensidades entre -80 y $-90 \mathrm{dBm}$ representadas en color ámbar. Lo mismo sucede en la zona norte en las proximidades de la Plaza de las Salelas y en el barrio de Los Barreros en la zona de la Media Sala. 

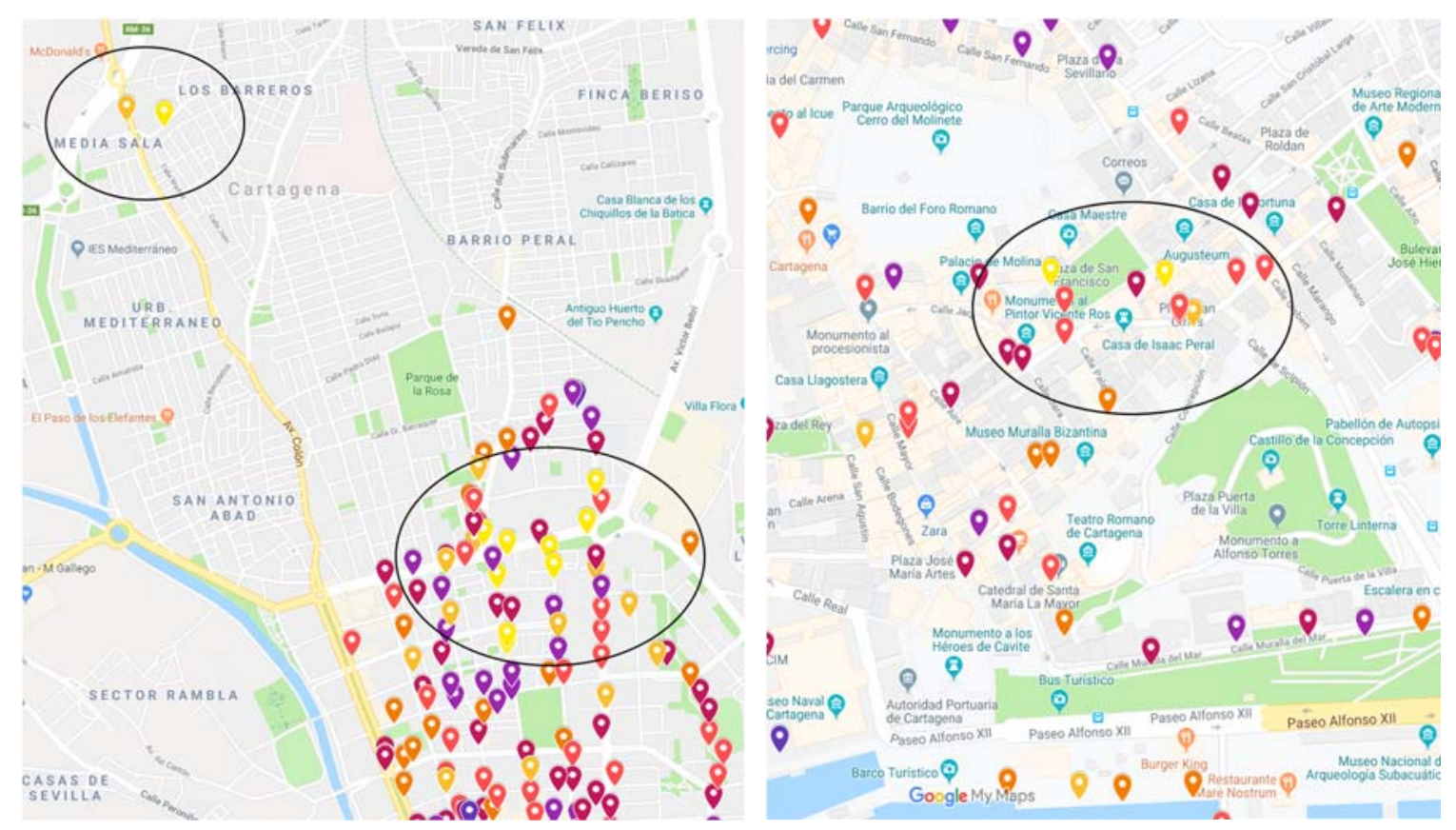

Figura 4.6. Zonas con mayor intensidad de señal en entorno urbano.

Medidas adicionales a pie de calle en cada zona y una exploración visual han servido para localizar las antenas correspondientes a las estaciones base que operan en dicha área. En el caso del casco antiguo, la estación base se encuentra en la terraza de un edificio de viviendas de cuatro plantas, junto a la fachada, y puede verse desde la calle. El identificador asociado es 1DD7 en base a la información devuelta por el backend en la trama de bajada. Incluye una antena de fibra de vidrio de color verde montada sobre mástil con un longitud aproximada de 1.5 metros que puede corresponder con un modelo de $6 \mathrm{dBi}$ de ganancia en la banda de $868 \mathrm{MHz}$. En el caso de la zona norte de la ciudad, con identificador 28E2, la antena de la estación base se localiza sobre un edificio de ocho plantas en un mástil al que se ha añadido un suplemento perpendicular, posiblemente para separar más la antena de la torreta del edificio y minimizar en lo posible el efecto de ésta en el lóbulo de radiación. Se trata de una unidad de fibra de vidrio de color blanco, puntera roja, base dorada y longitud inferior a $1 \mathrm{~m}$ que corresponde al modelo CLX900-3LW de Procom con 5 dBi de ganancia. Similar es la localizada en la barriada de Los Barreros con identificador 1002. En este caso se ubica en la torre de telecomunicaciones de un operador de telecomunicaciones y es compartida con una estación base de telefonía móvil y otros servicios. En la Figura 4.7 se sitúan las estaciones base de Sigfox localizadas en la ciudad de Cartagena. 


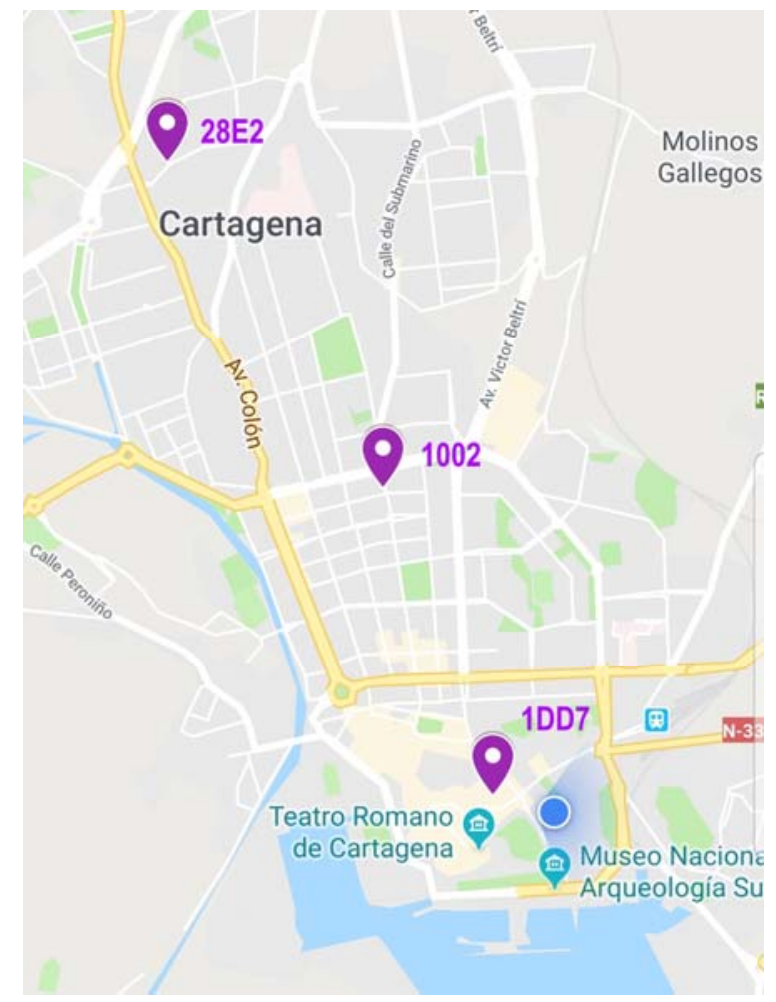

Figura 4.7. Ubicación de estaciones base Sigfox en entorno urbano.

En la Figura 4.8 puede verse una fotografía de la antena correspondiente a la estación base Sigfox de la zona norte de la ciudad. Comparte ubicación con dos BTS (Base Transceiver Station) de telefonía móvil, posiblemente gestionadas por el mismo operador (Cellnex Telecom). En la imagen puede apreciarse también el amplificador LNA intercalado en la línea coaxial de la antena y fijado cerca de ésta en el mismo soporte transversal.
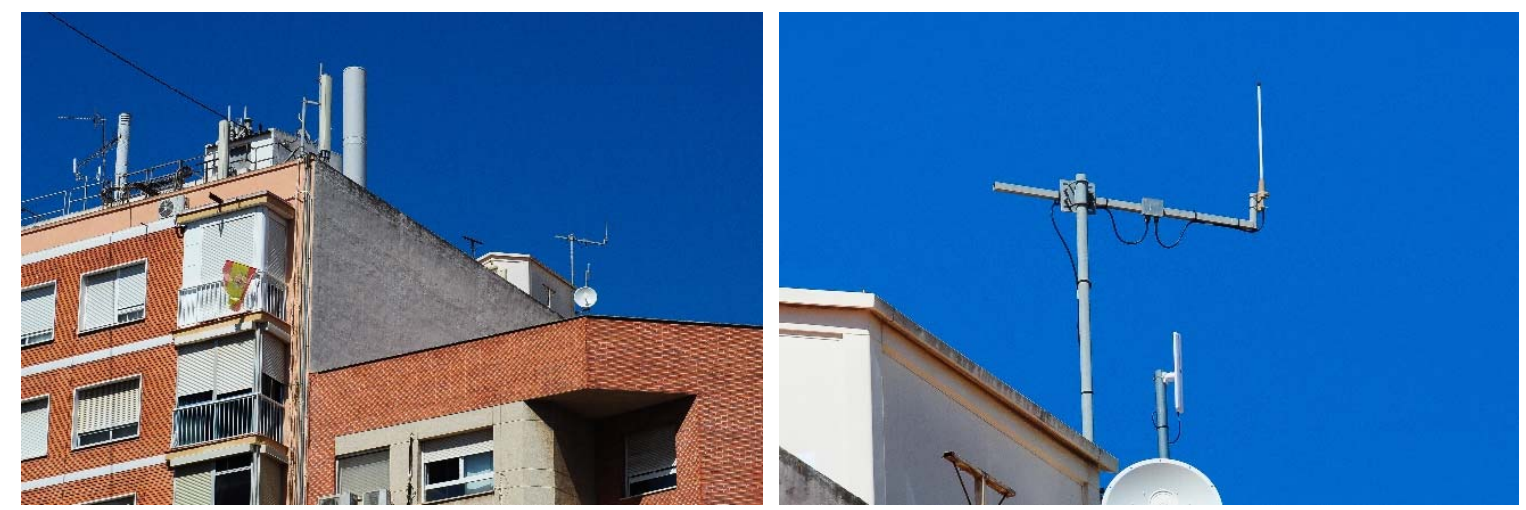

Figura 4.8. Imagen de la antena de una estación base Sigfox. 


\subsubsection{Estudio de cobertura LoRaWAN}

\subsubsection{Medidas en entorno urbano}

Para las pruebas LoRaWAN, al no haber ningún servicio establecido en la zona, ha sido necesitado crear una zona de cobertura propia. Para ello se ha configurado e instalado una estación base de exterior modelo Multitech Conduit IP67, a la que enlazarán los dispositivos de telemedida y telecontrol. Se elegido para su montaje una ubicación del casco urbano con la mayor altura posible y los menores obstáculos naturales y artificiales a su alrededor. La estación base ha sido instalada a 71 metros sobre el nivel del mar según un receptor GPS. En concreto, en la en la azotea del edificio ELDI de la Universidad Politécnica de Cartagena, donde su antena omnidireccional de fibra de vidrio y $3 \mathrm{~dB}$ de ganancia queda a la altura de una planta 5 , estando ya de por sí el edificio construido en una zona de la ciudad de natural elevación y despejada hacía el casco urbano y buena parte del campo de Cartagena como puede verse en la Figura 4.9. El único obstáculo orográfico cercano es el cerro de La Concepción, en dirección suroeste, pudiendo afectar a una zona muy reducida de la ciudad y el área militar del puerto.

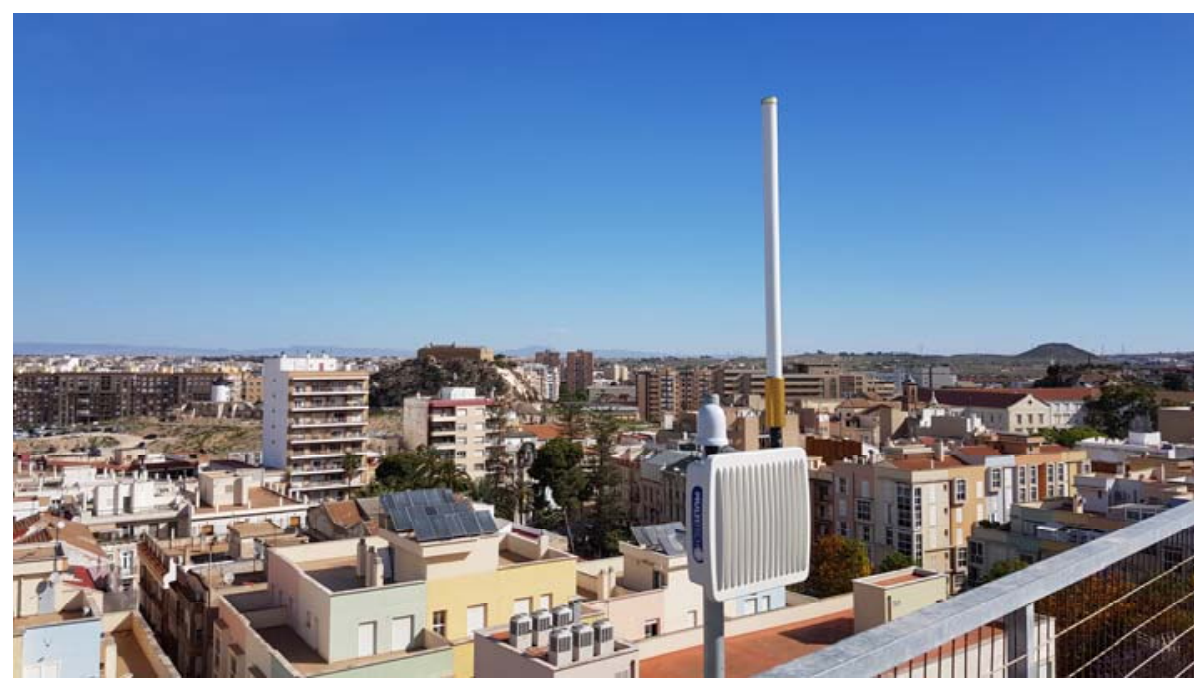

Figura 4.9. Estación base LoRaWAN instalada.

Para comprobar hasta qué distancia puede enlazarse con la estación base y los niveles de señal presentados, se han llevado a cabo medidas a nivel de calle con el medidor de campo Adeunis ARF8123A. En primer lugar, se ha verificado que en puntos a una distancia de $2 \mathrm{~km}$ y línea visual, es posible establecer enlace y el nivel de señal es del orden de $-97 \mathrm{dBm}$. En segundo lugar, se ha comprobado cobertura en el área suroeste de la ciudad, con posible zona de sombra provocada por el cerro de La Concepción. Sin embargo, el enlace es posible y los niveles de señal junto a edificios de cinco plantas son más que aceptables, del orden de $-117 \mathrm{dBm}$. Por último, se ha procedido a ir tomando distancia respecto a la estación base con el dispositivo de test en mano por calles de la ciudad flanqueadas por edificios de hasta ocho plantas. Puede verse en la Figura 4.10 como los niveles de señal medidos a una distancia de $1 \mathrm{~km}$ son de -110 $\mathrm{dBm}$ y van descendiendo hasta $-121 \mathrm{dBm}$ a una distancia de $2 \mathrm{~km}$, teniendo aún margen pues la sensibilidad del receptor es de $-140 \mathrm{dBm}$. En ningún momento se contaba con línea visual con la estación base ni ha habido ausencia de enlace en ninguno de los puntos verificados. 


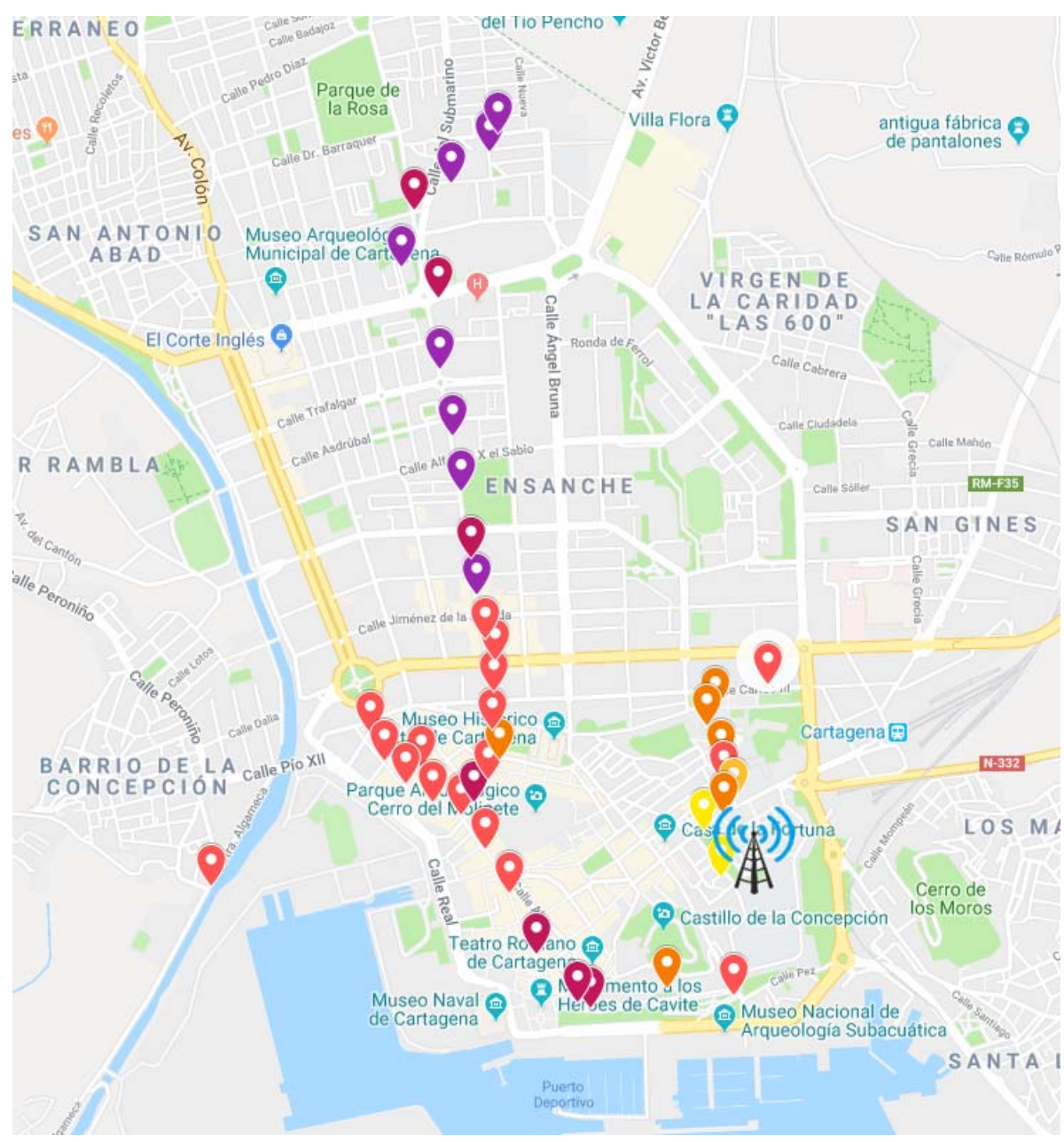

Figura 4.10. Medidas de cobertura LoRaWAN en Cartagena.

Se ha efectuado también pruebas en un entorno muy adverso, el interior del edificio de la Escuela de Ingeniería Industrial, con muros de piedra de 1 metro de espesor. Los niveles de señal son del orden de $-140 \mathrm{dBm}$ en la zona del hall, $-114 \mathrm{dBm}$ y $-123 \mathrm{dBm}$ en pasillos y escaleras respectivamente, y $-117 \mathrm{dBm}$ en los patios. Finalmente hay cobertura en la bajada al sótano pero no en el interior este.

\subsection{Validación en laboratorio}

La validación del sistema desarrollado se ha realizado en dos fases. Una primera fase se ha realizado a nivel de laboratorio. Se ha dispuesto de una maqueta como la representada en la Figura 4.11, la cual consta de un circuito cerrado de agua, electrobomba, contador residencial similar al que utiliza la compañía de distribución y un dispositivo generador de impulsos, el cual se conecta al contador, cuya salida iría conectada al conector de entrada del dispositivo de adquisición descrito en el capítulo 3.

En la Figura 4.11 se muestran también las versiones finales de cada uno de los diferentes dispositivos desarrollados y descritos en secciones anteriores que se han ido probando durante esta fase de laboratorio. 
El objetivo fundamental de esta fase de pruebas a nivel de laboratorio es verificar que la electrónica funcionaba correctamente y se capturaban todos los pulsos eléctricos suministrados por el contador y que la información se transmitía por red de forma adecuada, con objeto de comprobar que los dispositivos presentaban un comportamiento adecuado, tanto a nivel hardware como software y podrían considerarse prototipos finales completamente operativos.
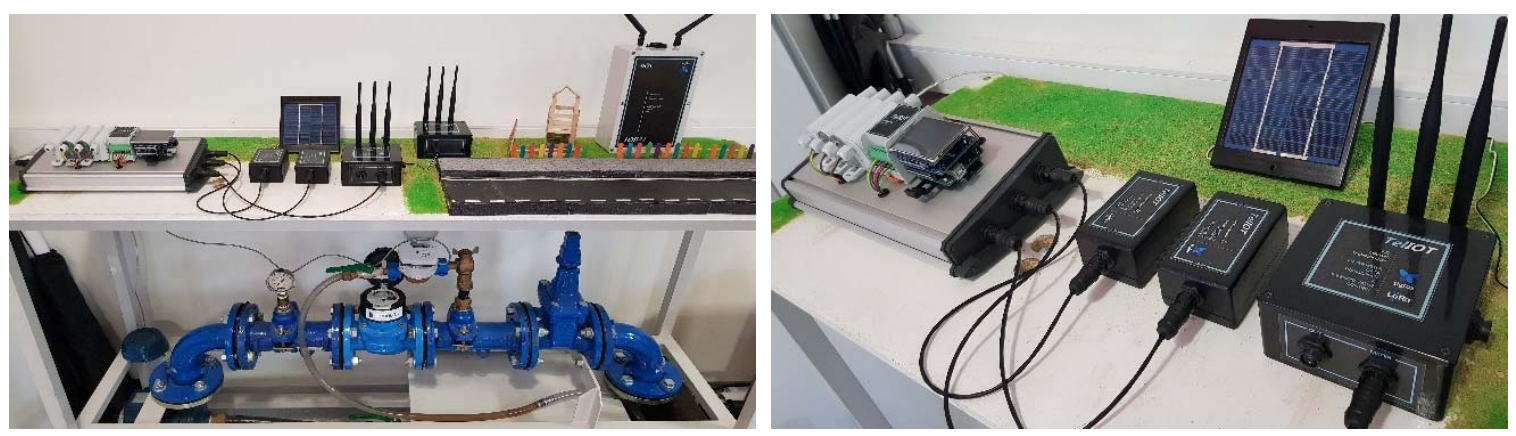

Figura 4.11. Entorno de pruebas con maqueta y dispositivos desarrollados.

Para llevar a cabo una simulación más en consonancia con los patrones de consumo domésticos, se ha controlado la electroválvula mediante un programador que provoca que haya circulación de agua a determinados intervalos del día y con diferentes duraciones.

\subsection{Casos de estudio en entornos reales}

\subsubsection{Prueba piloto en vivienda unifamiliar}

Se ha procedido a acoplar en el contador de consumo de agua de una vivienda unifamiliar un generador de impulsos y un emisor de datos con conectividad Sigfox con objeto de evaluar la tecnología en condiciones reales y a su vez llevar a cabo una recopilación de datos de consumo durante un periodo de tiempo de tres días. La instalación se ha llevado a cabo en el espacio de contador ubicado en la pared exterior de la vivienda y cerrado con una puerta metálica. Puede verse el conjunto de elementos en la Figura 4.12. Se ha configurado el dispositivo loT para una resolución de 1 litro y una transmisión de la información de consumo cada 30 minutos. Durante la prueba se ha solicitado a la familia que anotase el tipo de actividad de consumo de agua que se estaba realizando.

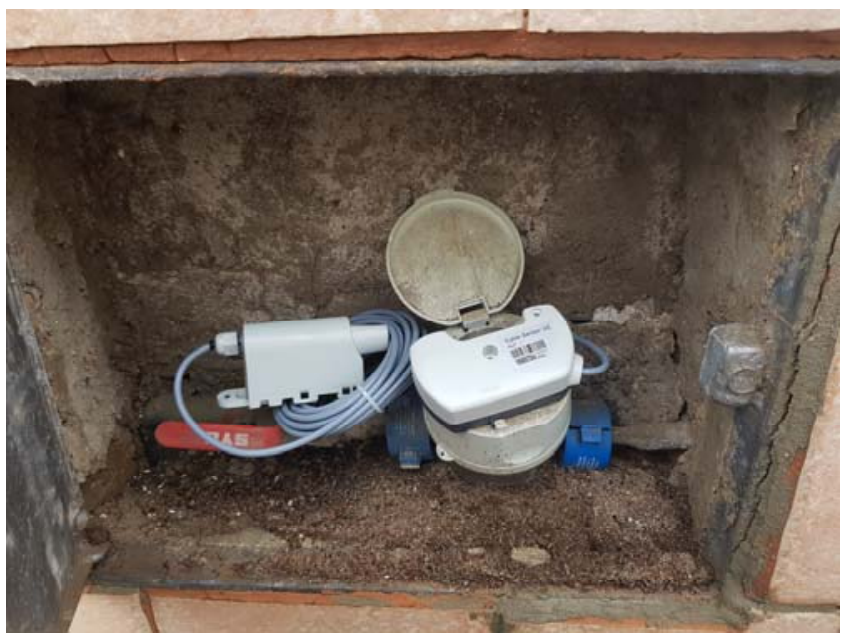

Figura 4.12. Medidas de cobertura LoRaWAN en Cartagena. 
En la Figura 4.13 se encuentran representados los datos de consumo de la vivienda recibidos durante los tres días de prueba. El comienzo y fin de la prueba se llevó a cabo al mediodía. Puede distinguirse a primera vista el periodo diurno de los tres días en los que hay mayor actividad en la vivienda y por tanto consumo, y los periodos nocturnos en los que disminuye el consumo. A pesar de ello se producen consumos debidos a la descarga de cisternas en aseos. Destaca un pico de consumo en la mañana del segundo día correspondiente a un sábado. Es posible que coincidieran varios consumidores tal como lavadora, lavaplatos, riego y/o duchas.

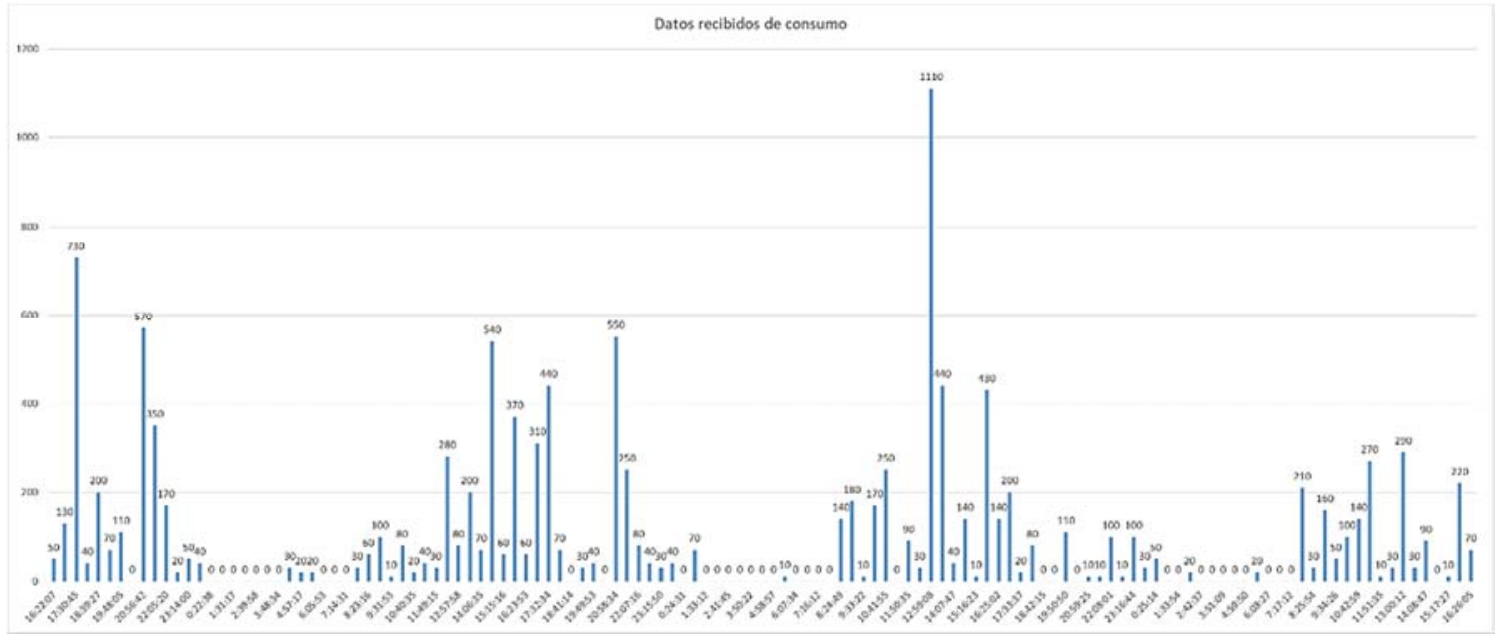

Figura 4.13. Datos de consumo obtenidos de vivienda unifamiliar.

En la Figura 4.14 puede verse detallado un día de consumo. Puede apreciarse como la actividad de consumo comienza en la mañana sobre las 7:30 horas, crece una hora más tarde con los desayunos y coincidiendo con aseos, se prolonga durante la mañana por consumo regular para beber y lavado de ropa, para intensificarse sobre las 12 horas con el inicio del cocinar. Sobre las 15 horas al finalizar la comida hay un pico de consumo por actividad en el fregador y puesta en funcionamiento del lavaplatos. Durante la tarde el siguiente pico de consumo corresponde al llenado de cubos para el fregado de la vivienda y al riego del jardín. Durante todo el día hay un consumo distribuido por descarga de cisternas y uso del lavabo en el aseo, así como de agua del grifo para beber. Destacar que durante dos tramos de lectura por la tarde, el consumo en esos intervalos es nulo. Por último, a la hora de la cena vuelve a haber un incremento por las mismas razones que en la comida, y posteriormente decrece hasta hacerse nulo a medianoche que los inquilinos se acuestan.

La capacidad de tener una evolución temporal de los datos de consumo durante todo el día con suficiente resolución permite adicionalmente la detección de fugas de agua en la instalación. En este caso concreto hay un periodo nocturno de 4.5 horas en el que el consumo acumulado se mantiene a cero. Teniendo en cuenta que la resolución del sistema es de 1 litro, esto significa que 0 no hay fugas de agua en absoluto, 0 que al menos, estas son inferiores a $1 \mathrm{~L}$ cada 4.5 horas. En caso contrario de existir una fuga considerable por avería en la instalación, podría detectarse inmediatamente en la evolución del consumo que este nunca llega a alcanzar el valor cero. Esto además podría correlacionarse con los patrones de consumo de esa vivienda, en los que aparecerán de manera repetitiva intervalos de tiempo diarios, especialmente por la noche, en los que el consumo suele ser cero. 


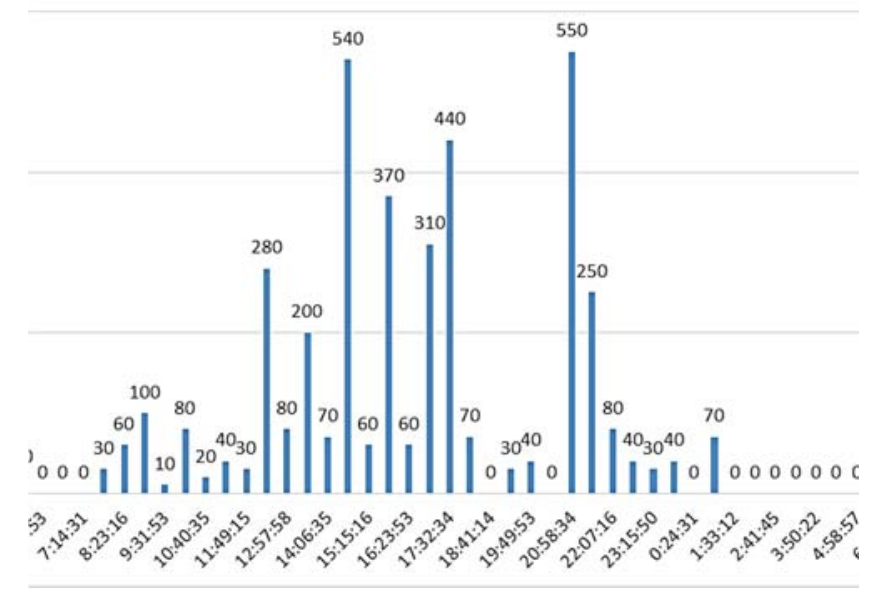

Figura 4.14. Datos de consumo en un día de vivienda unifamiliar.

\subsubsection{Instalación en finca rural}

Se ha querido comprobar la efectividad de estas tecnologías de comunicación loT fuera del entorno urbano donde la concentración de estaciones base que proporcionan cobertura siempre es mayor. En concreto se ha seleccionado una finca rural separada de cualquier núcleo de población y localizada a $28 \mathrm{~km}$ en línea recta del centro de la ciudad de Murcia, cerca de la pedanía de Campules. La localización concreta puede verse en el mapa de la Figura 4.15.

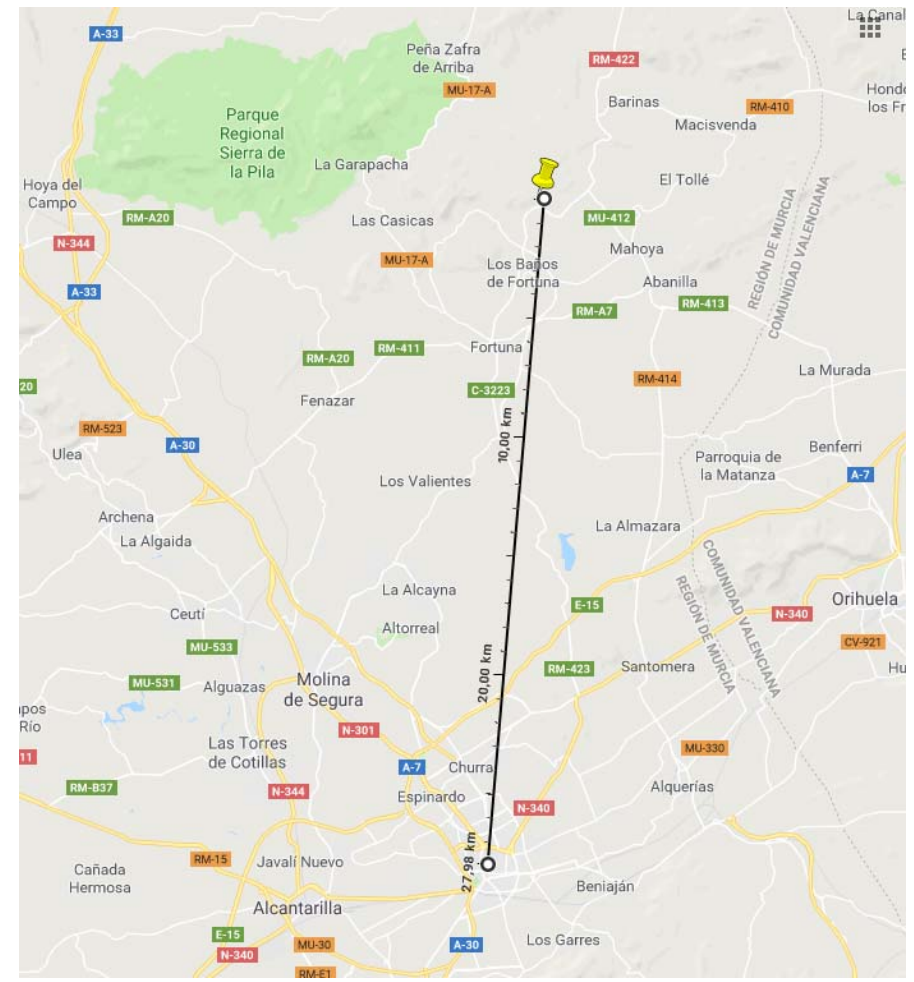

Figura 4.15. Ubicación de la instalación en finca rural.

A pesar de que en esta ubicación la cobertura de telefonía móvil 3G y $4 G$ es escasa, se ha podido comprobar usando el medidor de campo que no se presentaban problemas de enlace con la red Sigfox. En concreto la red recibió al dispositivo con $-101 \mathrm{dBm}$ de señal y en la pantalla del dispositivo se mostró un nivel de $-108 \mathrm{dBm}$ en la señal de bajada desde la red. 
Se procedió a acoplar al contador de consumo de agua de la finca de tipo Itron Flodis, un generador de pulsos Itron Cyble con $\mathrm{K}=1$ y un dispositivo Sigfox ARF8048PA de Adeunis configurado para la transmisión de datos cada 10 minutos. En la Figura 4.16 pode verse el montaje en el contador de agua de la finca, con el lector sobre el contador y el transmisor en la parte inferior derecha y tras la tubería metálica con objeto de forzar unas condiciones de comunicación RF aún más desfavorables.

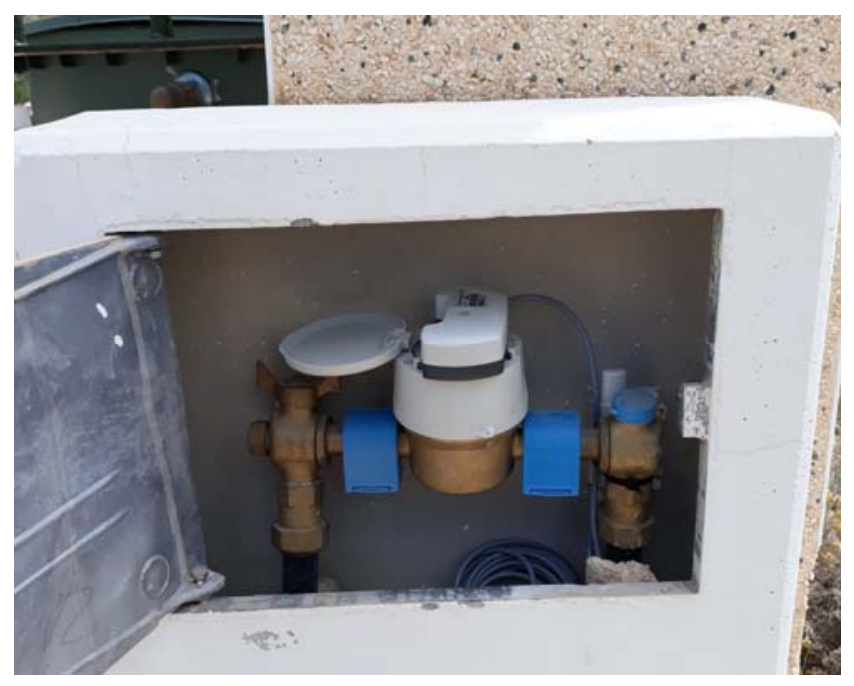

Figura 4.16. Instalación en finca rural.

A pesar de la localización en entorno rural apartada de núcleos de población, de la instalación en el interior de una caja de contador con puerta metálica y de haberse instalado el transmisor en una posición interior y desfavorable, se puede comprobar en el listado de los mensajes recibidos en el backend de Sigfox en la Figura 4.17, como la comunicación es factible y estable, no manifestándose perdida de datos en ningún momento. Destacar además el nivel de señal representado en los gráficos de barras con color verde y un nivel 4 de 5.

\begin{tabular}{|c|c|c|c|c|}
\hline Time & Data / Decoding & LQI & Callbacks & Location \\
\hline 2019-05-30 19:21:39 & 029006680600000000000000 & & & \\
\hline 2019-05-30 19:11:20 & 028006680600000000000000 & & & \\
\hline 2019-05-30 19:00:56 & 027006680600000000000000 & & & \\
\hline 2019-05-30 18:50:38 & 026006680600000000000000 & III & & \\
\hline $2019-05-3018: 40: 12$ & 025006680600000000000000 & III & & \\
\hline
\end{tabular}

Figura 4.17. Tramas recibidas de finca rural. 


\subsection{Despliegue real}

\subsubsection{Selección de ubicaciones}

Una vez que se ha confirmado en laboratorio el correcto funcionamiento de los dispositivos y que se ha verificado tanto su comportamiento hardware como que los datos se transmitían correctamente al sistema de información del usuario final, se pasó a la fase 2, consistente en el desarrollo de un caso de estudio en condiciones reales de funcionamiento. Para ello, se solicitó a una compañía distribuidora de Agua los datos asociados a diversos contadores que monitorizan de forma remota mediante tele-lectura. El sistema de tele-lectura utilizado por la compañía se basa en una comunicación mediante enlace radio de $169 \mathrm{MHz}$ en la Banda ISM. La antena de recepción se encuentra ubicada en la azotea de un edificio que la compañía tiene en la ciudad de Cartagena. El sistema de adquisición de la compañía se encuentra configurado de tal forma que captura y transmite una medida cada hora, por lo que una lectura de un día supondría la adquisición y transmisión de 24 medidas.

La empresa suministró las lecturas correspondientes a un periodo de 100 días de 78 contadores ubicados en diferentes zonas de la ciudad tomadas al azar. La Figura 4.18 resume los datos suministrados por estos contadores. Al ser lecturas correspondientes a 100 días, lo previsible es que se hubieran recibido 2400 muestras en total por cada contador (100 días x 24 muestras/día). Sin embargo, como se aprecia en la Figura 4.18, esto no ha ocurrido así para todos los contadores, lo que implica que hay problemas asociados a la toma de medidas. Se ha dividido el rango de muestras en tres zonas diferentes, utilizando para ello los cuartiles estadísticos Q1 (25\%) y Q3 (75\%). Se han clasificado como 'alarmantes' (marcados en rojo en la figura) todos aquéllos contadores de los que se han recibido una cantidad de medidas inferior al $25 \%$ de la cantidad esperada durante el periodo de lectura considerado. Se han clasificado como 'críticos' (marcados en amarillo en la figura) los contadores de los que se han recibido una cantidad de medidas comprendida entre el $25 \%$ y el $75 \%$ de la cantidad esperada. Finalmente, se han considerado como 'aceptables' (marcados en verde) aquellos contadores de los que se ha recibido una cantidad de medidas superior al $75 \%$ de la cantidad esperada.

Analizando 'a posteriori' la ubicación de los contadores, se ha comprobado que los contadores ubicados por debajo del cuartil $25 \%$ provienen en su mayoría de dispositivos colocados en sótanos de edificios, lo que hace pensar que las pérdidas vienen producidas fundamentalmente porque la señal radio tiene problemas para atravesar la estructura del edificio. Las lecturas procedentes de contadores ubicados entre las zonas de los cuartiles del $25 \%$ y $75 \%$ presentan una mayor diversidad en el origen. Algunos se encuentran ubicados en sótanos, con quizás una mejor cobertura y otros se encuentran ubicados en plantas bajas o entradas de viviendas residenciales a la intemperie, lo que hace pensar que quizás se encuentran en zonas de sombra de recepción debidas al hecho de encontrarse en un entorno urbano en el que la cobertura puede presentar una cierta variabilidad.

Conviene recalcar que la compañía realiza una campaña periódica en la que personal cualificado pasa por cada vivienda tomando lectura manual del valor del contador, por lo que a efectos de facturación esta pérdida de muestras no influye en la generación de la factura de cara al cliente y no tiene impacto económico en lo facturado como servicio por la compañía distribuidora. Sin embargo, estas pérdidas de datos sí influyen de cara a la parte estratégica y operativa de la compañía, no sólo porque implica que es necesario mantener una persona dedicada a la lectura manual, sino que también imposibilita el poder crear en algunas viviendas una estrategia que permita estimar los hábitos de consumo de los usuarios, ya que, aunque los actuales contadores 
están configurados para muestrear cada hora, podrían ser configurados para tener un tiempo de muestreo de hasta 15 minutos.

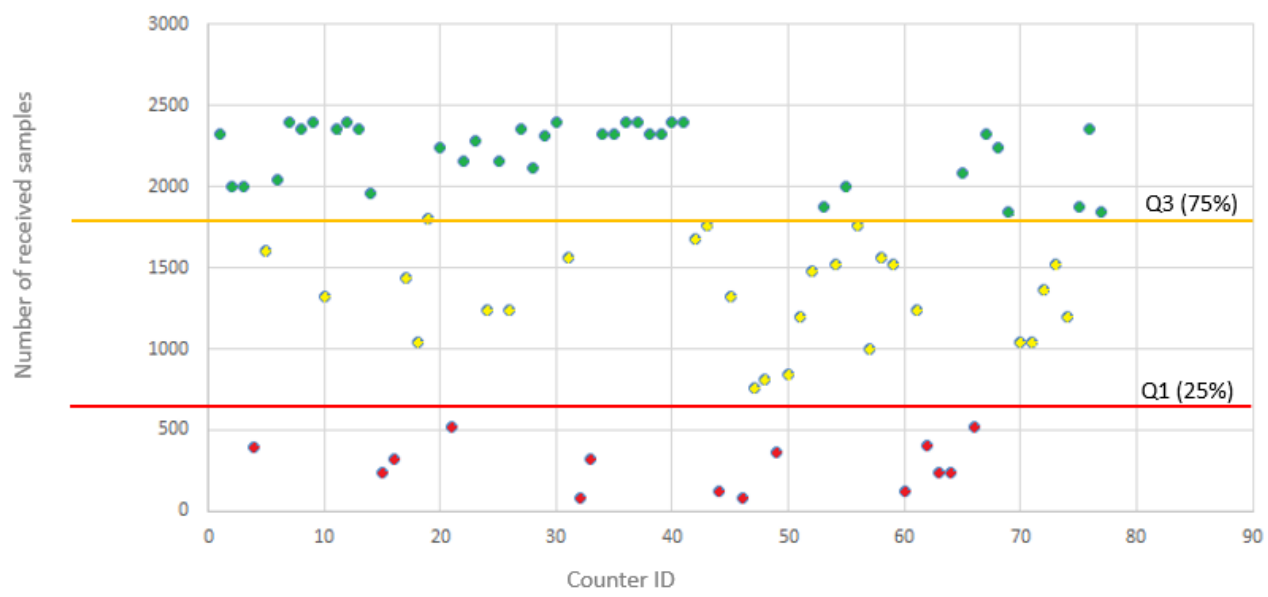

Figura 4.18. Muestras procedentes de 78 contadores seleccionados al azar.

\subsubsection{Descripción del despliegue}

Se ha diseñado un caso de estudio en el que se han seleccionado 5 edificios en los que se dispone de contadores con una tasa de recepción de muestras inferior al 25\%. En estos edificios, en paralelo con el contador de la empresa suministradora, se ha colocado un prototipo de cada clase (Sigfox, LoRaWAN y LoRa-Sigfox Gateway). En 3 edificios con contadores ubicados en zonas críticas (25\%-75\%) se ha colocado también un prototipo de cada clase. Finalmente, en 3 edificios ubicados en las zonas aceptables se han colocado solamente un dispositivo Sigfox y otro LoRaWAN. En el despliegue de la instalación, se ha dispuesto una estación base LoRaWAN en la azotea de un edificio equidistante a la ubicación de todos los anteriores. En los edificios en los que se ha colocado un dispositivo LoRa-Sigfox Gateway, éste se ha colocado en un patio de luces de la vivienda. La Tabla 4.4 resume la estructura del despliegue.

\begin{tabular}{|c|c|c|c|c|}
\cline { 2 - 5 } \multicolumn{1}{c|}{} & Sigfox & LoRaWAN & $\begin{array}{c}\text { LoRa-Sigfox } \\
\text { Gateway }\end{array}$ & $\begin{array}{c}\text { Edificios } \\
\text { Seleccionados }\end{array}$ \\
\hline Alarmantes & $\checkmark$ & $\checkmark$ & $\checkmark$ & 5 \\
\hline Críticos & $\checkmark$ & $\checkmark$ & $\checkmark$ & 3 \\
\hline Aceptables & $\checkmark$ & $\checkmark$ & & 3 \\
\hline
\end{tabular}

Tabla 4.4. Tipo de tecnología en cada edificio seleccionado.

En total, se han colocado 30 prototipos: 11 contadores Sigfox, 11 contadores LoRaWAN y 8 contadores con LoRa-Sigfox Gateway. En paralelo a cada uno de ellos, se ha dejado tomando muestras un contador de los que actualmente utiliza la compañía. Se ha estado monitorizando el comportamiento del despliegue durante 10 días consecutivos. Los resultados del caso de estudio son los que se describen a continuación.

La Figura 4.19 muestra una gráfica sobre la lectura de contadores obtenida durante un periodo de 10 días (lo que equivaldría a 240 muestras) recibidos por cada uno de los diferentes 
dispositivos en el caso de los denominados 'alarmantes' (Q1<25\%). Este contador se encuentra ubicado en el sótano de un edificio de viviendas.

ISM (56 samples received)

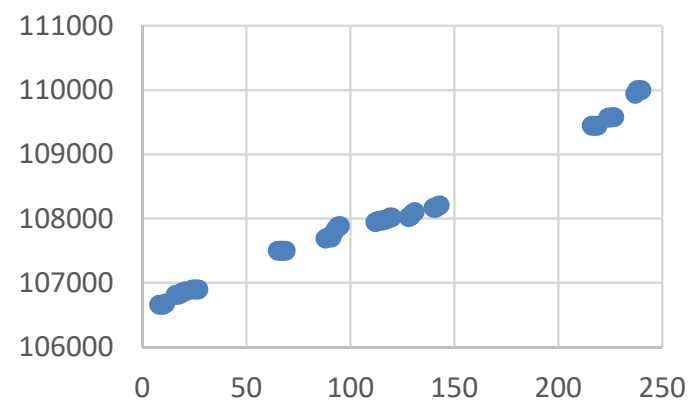

(a)

\section{LoRaWAN (213 samples received)}

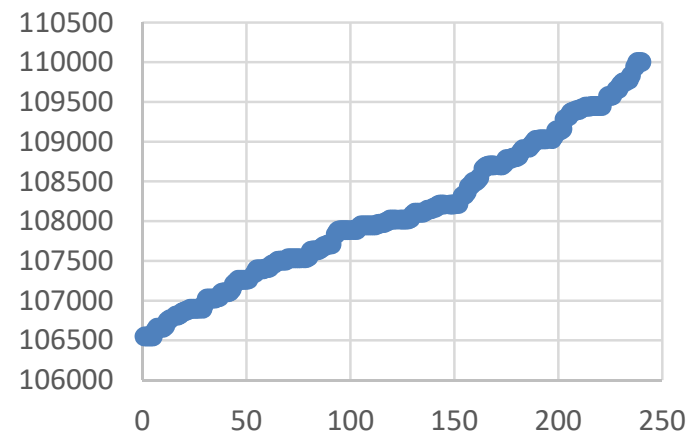

(c)
Sigfox (134 samples received)

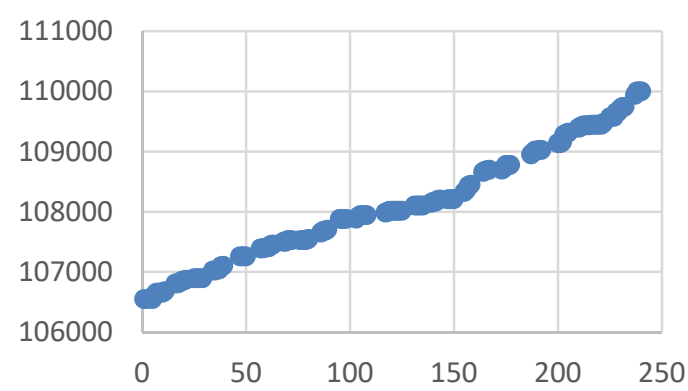

(b)

Lora-Sigfox Gateway

(240 samples received)

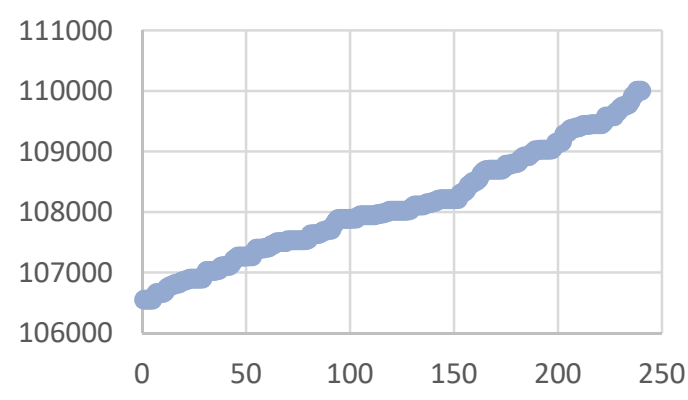

(d)

Figura 4.19. Muestras recibidas en el caso tasa de recepción por debajo del 25\%.

Como se puede observar en la Figura 4.19, en la banda ISM se cumple lo que se preveía. La tasa de recepción de muestras, sobre un esperado de 240 , sólo es de 56 , lo que significa un $25 \%$ de porcentaje de llegadas. Con el prototipo Sigfox se ve una mejora en la tasa de recepción, alcanzando las 134 muestras recibidas (55.8\%). El prototipo LoRaWAN recibe durante el periodo 213 muestras (88.8\%). La estación base LoRaWAN se encuentra situada más cercana al contador que la estación Sigfox, cuya ubicación viene marcada por la elegida por el operador comercial para la ciudad de Cartagena. Los datos obtenidos para este contador parecen indicar que el problema asociado a la recepción viene asociado a que la señal no tiene potencia suficiente para traspasar hasta la ubicación física del contador. Como se puede observar, la solución con un prototipo LoRa-Sigfox Gateway permite obtener una potencia de señal que permita penetrar y alcanzar el sótano del edificio, logrando una tasa de recepción del $100 \%$.

La Tabla 4.5 resume los resultados del caso de estudio para cada uno de los diferentes edificios con contadores ubicados en la franja de recepción menor del 25\% en la banda ISM. Observando los datos, se comprueba que la franja ISM sigue presentando pérdidas de datos en la recepción. Las soluciones basadas en Sigfox y LoRaWAN mejoran la recepción, estando la recepción LoRaWAN, con estación base colocada más cercana al contador que la Sigfox, con una tasa de recepción mayor, lo que parece reforzar la idea de que las pérdidas vengan debidas a que la 
señal de radio no es capaz de alcanzar la ubicación del contador. Finalmente, en la solución LoRa-Sigfox Gateway sí se consigue alcanzar una recepción del 100\%. En consecuencia, para contadores situados en esta franja en la que la recepción es inferior al 25\%, la solución LoRaSigfox Gateway parece ser la más indicada si se desea obtener una tasa de recepción del 100\%.

\begin{tabular}{|c|c|c|c|c|}
\hline Edificio & ISM & Sigfox & LoRaWAN & $\begin{array}{c}\text { LoRa-Sigfox } \\
\text { Gateway }\end{array}$ \\
\hline B01 & $56(23 \%)$ & $134(55.8 \%)$ & $213(88.8 \%)$ & $240(100 \%)$ \\
\hline B02 & $36(15 \%)$ & $101(42.1 \%)$ & $166(69.2 \%)$ & $240(100 \%)$ \\
\hline B03 & $32(13.3 \%)$ & $92(38.3 \%)$ & $173(72.1 \%)$ & $240(100 \%)$ \\
\hline B04 & $41(17.08 \%)$ & $110(45.8 \%)$ & $188(78 \%)$ & $240(100 \%)$ \\
\hline B05 & $24(10)$ & $89(37.1 \%)$ & $146(60.1 \%)$ & $240(100 \%)$ \\
\hline
\end{tabular}

Tabla 4.5. Tasas de recepción de contador con un porcentaje inferior al 25\%.

La Figura 4.20 muestra una gráfica sobre la lectura de contadores obtenida durante el mismo periodo de 10 días (240 muestras) recibidos por cada uno de los diferentes dispositivos en el caso de los denominados 'críticos' (recepción entre el 25\% y el 75\%). Este contador se encuentra ubicado en el sótano de un edificio de viviendas.

ISM (121 samples receives)

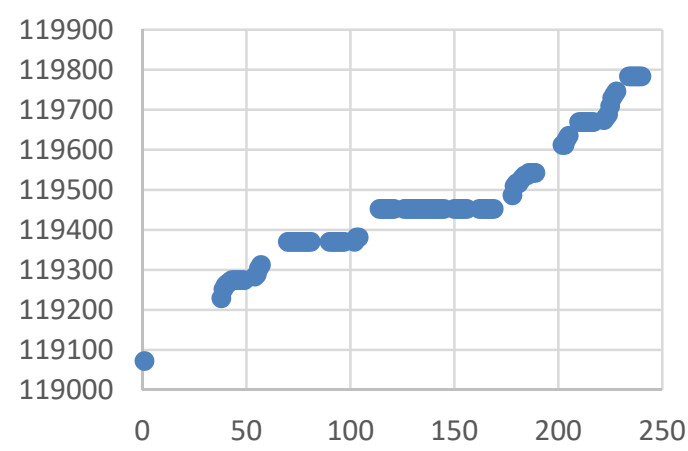

(a)

LoRaWAN (240 samples received)

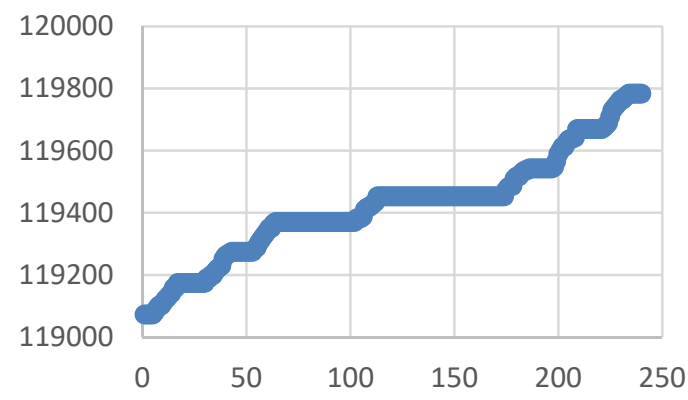

(c)

\section{Sigfox (218 samples received)}

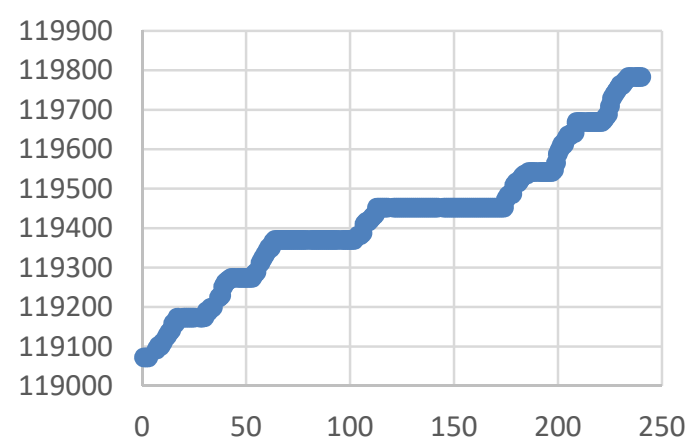

(b)

Lora-Sigfox Gateway

(240 samples received)

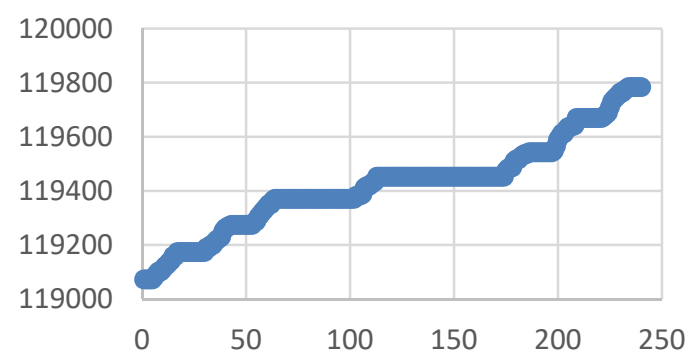

(d)

Figura 4.20. Número de muestras recibidas con tasa de recepción entre 25\% y 75\%.

Como se puede observar en la figura, en la banda ISM la tasa de recepción de muestras, sobre un esperado de 240 es de 121, lo que significa un 50.4\% de porcentaje de llegadas. Con el prototipo Sigfox se ve una mejora en la tasa de recepción, alcanzando las 218 muestras 
recibidas (90.8\%). En esta ocasión, tanto el prototipo basado en LoRaWAN como el prototipo LoRa-Sigfox Gateway permite obtener una potencia de señal que permita penetrar y alcanzar el sótano del edificio, logrando una tasa de recepción del 100\%. En este caso, parece que como la estación base LoRaWAN se encuentra más cercana al contador que la Sigfox, la cobertura LoRaWAN asegura una adecuada tasa de recepción.

La Tabla 4.6 resume los resultados del caso de estudio para cada uno de los diferentes edificios con contadores ubicados en la franja de recepción entre el $25 \%$ y el $75 \%$ en la banda ISM. Se puede observar que Sigfox mejora la tasa de recepción, llegando en algún caso a alcanzar el 100\%. Sin embargo, son las soluciones basadas en LoRaWAN y LoRa-Sigfox Gateway las que permiten alcanzar una tasa de recepción del 100\%. En el caso de LoRaWAN, posiblemente sea debido a que la estación base se encuentra ubicada más cercana que la Sigfox, prefijada por la ubicación seleccionada por el operador de la zona.

\begin{tabular}{|c|c|c|c|c|}
\hline Edificio & ISM & Sigfox & LoRaWAN & $\begin{array}{c}\text { LoRa-Sigfox } \\
\text { Gateway }\end{array}$ \\
\hline B06 & $121(50.4 \%)$ & $218(90.8 \%)$ & $240(100 \%)$ & $240(100 \%)$ \\
\hline B07 & $148(61.7 \%)$ & $229(95.4 \%)$ & $240(100 \%)$ & $240(100 \%)$ \\
\hline B08 & $170(70.8 \%)$ & $240(100 \%)$ & $240(100 \%)$ & $240(100 \%)$ \\
\hline
\end{tabular}

Tabla 4.6. Tasas de recepción en contador entre el $25 \%$ y el $75 \%$.

La Figura 4.21 muestra una gráfica sobre la lectura de contadores obtenida durante el mismo periodo de 10 días (240 muestras) recibidos por cada uno de los diferentes dispositivos en el caso de los denominados 'aceptables' (recepción superior al 75\%). Este contador se encuentra ubicado en la arqueta de entrada a una vivienda residencial (chalet), similar al de la figura 20.c.

ISM (217 samples received)

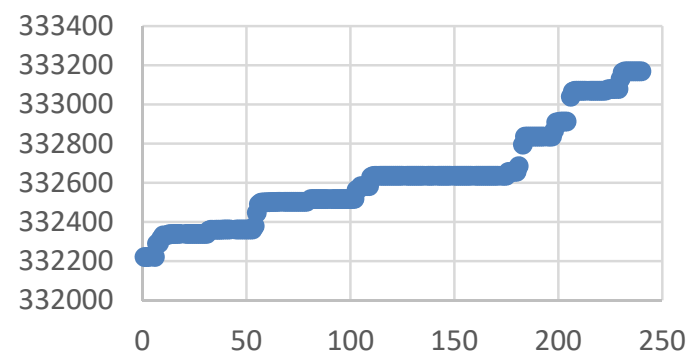

(a)

\section{LoRaWAN (240 samples received)}

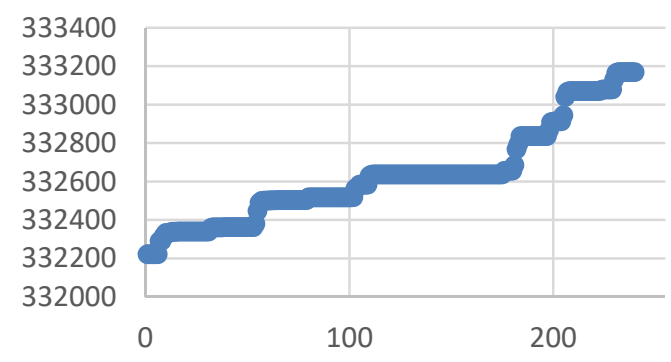

(c)
SigFox (240 samples received)

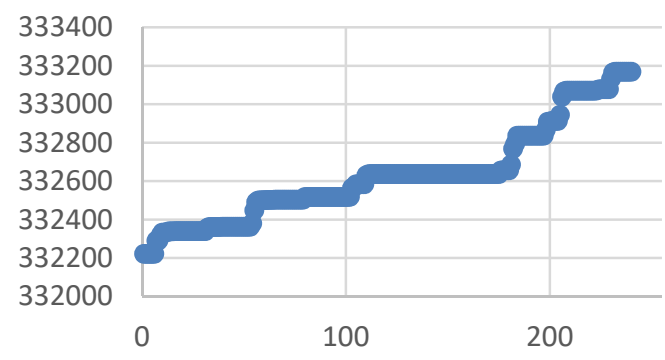

(b)

Lora-Sigfox Gateway

(240 samples received)

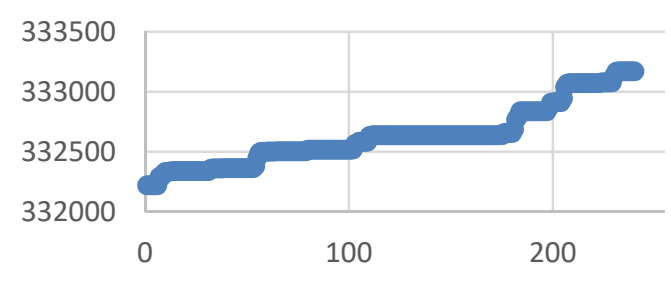

(d)

Figura 4.21. Muestras recibidas en el caso de tasa de recepción superior al 75\%. 
Como se puede observar en la Figura 4.21, en la banda ISM la tasa de recepción de muestras, sobre un esperado de 240 es de 217, lo que significa un 90.4\% de porcentaje de llegadas. En esta ocasión, las tres alternativas de prototipos consiguen obtener una tasa de llegada del $100 \%$. La Tabla 4.7 resume los resultados del caso de estudio para cada uno de los diferentes edificios con contadores ubicados en la franja de recepción superior al $75 \%$ en la banda ISM. En esta ocasión, los tres prototipos desarrollados alcanzan una tasa de recepción del 100\%, lo que indica claramente que si no hay zonas de sombra, las tecnologías loT mejoran las prestaciones de soluciones clásicas como las ISM utilizadas por el distribuidor de agua.

\begin{tabular}{|c|c|c|c|c|}
\hline Edificio & ISM & Sigfox & LoRaWAN & $\begin{array}{c}\text { LoRa-Sigfox } \\
\text { Gateway }\end{array}$ \\
\hline B09 & $217(90.4 \%)$ & $240(100 \%)$ & $240(100 \%)$ & $240(100 \%)$ \\
\hline B10 & $187(77.9 \%)$ & $240(100 \%)$ & $240(100 \%)$ & $240(100 \%)$ \\
\hline B11 & $198(82.5 \%)$ & $240(100 \%)$ & $240(100 \%)$ & $240(100 \%)$ \\
\hline
\end{tabular}

Tabla 4.7. Tasas de recepción en contador con porcentaje superior al 75\%. 


\subsection{Conclusiones}

Se ha podido verificar que en el núcleo urbano de la ciudad de Cartagena, a nivel de calle entre edificios, hay presencia de cobertura de la red Sigfox. Incluso se ha podido comprobar que esta es utilizable en localizaciones adversas como sótanos e interior de edificios con gruesos muros. La disponibilidad de servicio se ha comprobado también en entorno rural alejado de poblaciones.

Se ha verificado que con la instalación de una única estación base de tipo LoRaWAN en una ubicación favorable de la ciudad, la cobertura de ésta se encuentra presente en gran parte del núcleo urbano con niveles aceptables hasta una distancia de $2 \mathrm{~km}$ de la estación base.

Respecto al despliegue llevado a cabo, se puede concluir lo siguiente:

En las ubicaciones en las que la cobertura en la banda ISM tiene una tasa de recepción inferior al 25\%, parece que una solución como la descrita en el prototipo LoRa-Sigfox Gateway son las que permiten asegurar una tasa de recepción óptima. Las tecnologías Sigfox o LoRaWAN mejoran la recepción, pero no permiten alcanzar el 100\%, posiblemente por pérdidas en la potencia de la señal que no permiten la penetración hasta la ubicación de los contadores.

En las ubicaciones en las que la tasa de recepción en la banda ISM se encuentra entre el 25\% y el $75 \%$, los prototipos LoRa-Sigfox y LoRaWAN son los que ofrecen mayores prestaciones. Esto parece indicar que la comunicación con la estación Sigfox, cuya ubicación se encuentra predeterminada por el operador de red de la zona, en ocasiones no tiene la potencia suficiente para penetrar hasta la ubicación del contador. Por ello, habría que valorar o bien, colocar una estación base en alguna azotea de un edificio dentro del radio de acción de los contadores y luego colocar un dispositivo de adquisición LoRaWAN en cada contador, o bien acudir a la estrategia de colocación de 2 dispositivos (un dispositivo de adquisición LoRa-SigFox Gateway + el propio Gateway colocado en un patio de luces de la vivienda, que transmita la señal hasta una zona donde haya visibilidad Sigfox y se pueda comunicar con la estación del operador de red local), evitando así tener que ubicar una estación base LoRaWAN en una azotea. En las zonas en las que la cobertura la tasa de recepción en la banda ISM es superior al $75 \%$ los tres prototipos permiten alcanzar una tasa de recepción del 100\%. En este caso, la solución más adecuada parecería ser la solución basada en el prototipo Sigfox, ya que no sería necesario desplegar ninguna estación base adicional, tal y como implicaría la solución basada en el prototipo LoRaWAN, y sólo sería necesario un dispositivo frente a los dos que implicaría la solución basada en el prototipo LoRa-SigFox Gateway.

Una mejora adicional frente a la solución dada por el dispositivo en banda ISM es que los tres prototipos desarrollados permitirían alcanzar una tasa de muestreo cada 10 minutos, frente a la resolución de 15 minutos que ofrece la solución ISM, mejorando así no sólo la tasa de recepción, sino también ampliando la resolución del muestreo.

Las soluciones propuestas permitirían evitar la necesidad de tener personal cualificado revisando mensualmente todos los contadores, siendo sólo necesario su actuación en casos puntuales 0 en situaciones periódicas de revisión con objeto de confirmar el correcto funcionamiento de las instalaciones.

Finalmente, permitiría a la compañía distribuidora tener un mayor número de medidas sobre el que se podrían aplicar técnicas Big Data con objeto de estimar los hábitos de consumo de los usuarios y prever picos de consumo futuros, mejorando las estrategias de mantenimiento y ampliación de la red de distribución de la compañía. 
CAPÍtULO5

CONCLUSIONES 



\subsection{Conclusiones y contribuciones}

Este trabajo de Tesis Doctoral con un enfoque innovador se ha centrado en diseñar, desplegar y verificar el correcto funcionamiento de una arquitectura de red loT mixta aplicada al desarrollo de sistemas de telelectura inteligente. Se describen a continuación las conclusiones más relevantes alcanzadas durante el desarrollo de la misma.

Del estudio de la tecnología existente se extrae que una implementación de lectura automatizada en el servicio de suministro de agua requiere contadores de consumo pre-equipados con una salida digital de la información o el uso de un elemento adicional acoplado que genere dicha información a partir de la detección de la actividad del contador. La información de consumo se presenta como una salida pulsante que corresponde a una revolución de la aguja o dígito de menor peso, o bien, un múltiplo de estos. Los dispositivos disponibles comercialmente para la transmisión de información empleando tecnologías específicas loT permiten una rápida puesta en marcha si las condiciones son favorables, sin embargo, se trata de productos cerrados de prestaciones concretas, con capacidades de configuración limitadas, no económicos, sin posibilidad de ampliación o personalización, con una vida útil determinada por su batería integrada no recargable y con capacidad de conexión a un único tipo de red loT.

Se ha identificado como una prestación adicional innovadora y que crea un valor añadido a los nodos finales, la capacidad de actuar de manera remota sobre un elemento externo como una electroválvula. De esta manera, la compañía encargada del servicio sería capaz no sólo de tener información de consumo, sino de decidir sobre el suministro al abonado final en cada vivienda. El estudio de las posibilidades de las diferentes tecnologías LPWAN para IOT ha revelado que la capacidad de telecontrol es posible pero limitada. Esto es debido a dos condicionantes. En primer lugar al empleo de bandas ISM con limitaciones impuestas por regulación en la ocupación del canal. En concreto, en $868 \mathrm{MHz}$ un mismo dispositivo no puede transmitir en el mismo segmento en un intervalo inferior a 10 minutos. Por otra parte, en el caso particular de Sigfox, a diferencia de LoRaWAN, la licencia del servicio sólo permite 4 mensajes de bajada hacía el dispositivo al día. Esto condiciona las aplicaciones de telecontrol si se requiere una actuación inmediata, pero sigue siendo aceptable para el caso de control del suministro de agua a un abonado.

Previo a la implementación de cualquier solución, se ha verificado sobre el terreno la capacidad de ofrecer un área de cobertura extensa por parte de las tecnologías de comunicación LPWAN para IOT. Para ello, se ha realizado un estudio de cobertura de red de ambas tecnologías en la ciudad de Cartagena. En concreto, en el caso de Sigfox se ha comprobado que en el núcleo urbano de una ciudad, a nivel de calle entre edificios de ocho plantas, hay presencia de señal de la red y capacidad de enlace. Incluso se ha podido comprobar que esta es utilizable en localizaciones adversas como sótanos e interior de edificios con gruesos muros. Así mismo, la disponibilidad del servicio se ha verificado también en un entorno rural alejado de núcleos de población aún en el caso del interior de una caja de contador con puerta metálica. En el caso de una red de tipo LoRaWAN, se ha comprobado que con la instalación de una única estación base en una ubicación favorable de la ciudad, la cobertura de ésta se encuentra presente en gran parte del núcleo urbano con niveles aceptables hasta una distancia medida experimentalmente de $2 \mathrm{~km}$ de la estación base.

Ambas tecnologías LPWAN para IoT, Sigfox y LoRa, comparten el enfoque de habilitar la conectividad de objetos que requieren transmitir poca cantidad de datos no de forma continua, precisan una gran autonomía y enlazar a largas distancias en condiciones desfavorables. Sin embargo, el modelo de uso y negocio asociado es completamente diferente. Sigfox actúa como 
un operador de red y los dispositivos, una vez activados, proceden a utilizar una red ya desplegada y con amplia cobertura internacional. Esta tecnología, a pesar de suponer un gasto en forma de licencia anual por cada dispositivo que se quiera conectar, es adecuada en casos en los que se requiera una puesta en marcha rápida o no compense el montar una red propia de otra tecnología. Hay que considerar que no supone más gastos que los de conectividad, es el operador el que se encarga del mantenimiento, ampliación y gestión de la red para garantizar su disponibilidad. La información puede finalmente transferirse a la infraestructura del usuario. En el caso de LoRaWAN, es el propio usuario el que ha de establecer y gestionar toda la infraestructura de red, desde estaciones base distribuidas en localizaciones estratégicas para garantizar comunicación en el area de cobertura deseada, como de los servidores de red para permitir el registro y autenticación de dispositivos, así como la gestión de la información enviada por los dispositivos finales. Se presenta como una solución adecuada en el caso de que se desee un control absoluto sobre todos los elementos de la red y mayores posibilidades de personalización, así como cuando se prefiera una inversión inicial mayor para la instalación y puesta en marcha, pero un gasto periodico posterior limitado al mantenimiento de la misma. Como puede verse, cada una de estas tecnologías tiene sus ventajas e inconvenientes y de manera habitual es necesario tener que elegir entre ambas para llevar a cabo un despliegue de dispositivos IOT en cada caso particular. Sin embargo, la arquitectura loT propuesta en este trabajo permite combinar el empleo de ambas en una misma red IOT, seleccionando la más adecuada en cada caso o area de despliegue, aprovechando las virtudes de cada una y haciendo que la información fluya desde el usuario hasta el proveedor de servicios de forma transparente e independiente de la tecnología de conectividad empleada. Se trata por tanto de una solución con una relación coste/eficiencia óptima con posibilidad de adaptación a las necesidades del usuario.

Se ha diseñado una arquitectura de red mixta y flexible, capaz de combinar diferentes soluciones de comunicación IoT, que permite la conexión de objetos IoT sin la restricción de la tecnología empleada y capaz de adaptarse durante el despliegue a las necesidades de cada aplicación. Con esta solución se habilita la coexistencia tanto de dispositivos Sigfox como LoRaWAN y queda abierta la posibilidad de otras tecnologías. Además se facilita la interoperabilidad tanto de los dispositivos desarrollados en este trabajo, como los comercialmente disponibles aunque provengan de diferentes fabricantes. Se ofrece una solución innovadora y de bajo coste para ampliar el área de cobertura de la red a zonas de sombra en ubicaciones adversas utilizando también tecnologías loT.

Se han desarrollado y construido dispositivos hardware propios para su integración en la arquitectura propuesta con objeto de satisfacer las necesidades que se han ido presentando en el despliegue de la red y que no estaban cubiertas por productos ya existentes. Para el dispositivo de telemedida y telecontrol se han desarrollado dos versiones, llevando a cabo una optimización del mismo tanto en tamaño físico como en coste. A diferencia de las soluciones comerciales dispone de conectividad múltiple, tanto a redes LoRaWAN, como a Sigfox, así como al gateway propio. La trama de información puede ser personalizada según el caso particular de aplicación. Otra capacidad diferenciadora es la posibilidad de recargar su batería interna, no teniendo que ser desechado el producto o suponer costosas intervenciones. Se han seleccionado además conectores roscados de tipo industrial que a diferencia de los comercialmente disponibles, evitan la apertura del dispositivo para conexión del cableado a una regleta interna.

Como elementos integrantes de la red que faciliten el transporte de la información se han desarrollado también los siguientes dispositivos. El denominado gateway loT es un dispositivo intermedio que situado en una ubicación estratégica y haciendo uso de un enlace LoRa, permite la comunicación con el dispositivo de contador en zonas de sombra de cobertura Sigfox como 
sótanos u otras localizaciones adversas para recibir la señal de la red. Su capacidad de abastecimiento por energía fotovoltaica le dota de una elevada autonomía. Otro elemento de red propio ha sido la estación base LoRaWAN para exteriores basada en hardware y software abiertos y con coste inferior al de soluciones comerciales que ofrecen similar funcionalidad. Para facilitar el estudio de cobertura se ha construido un dispositivo con funciones no incluidas en productos comerciales. De manera adicional y como asistencia en el desarrollo de este trabajo, se ha montado una consola de pruebas en bastidor para la fase de validación en laboratorio que incluye posibilidad de recibir la señal de un contador o emularlo, replicarla y enviarla mediante transmisores de las diferentes tecnologías loT.

Tras llevar a cabo un despliegue en entorno urbano y con respecto a un sistema de telelectura en servicio en banda ISM de $169 \mathrm{MHz}$, se ha mejorado la tasa de recepción de datos aplicando tecnologías LPWAN para IoT. En ubicaciones con una tasa de recepción no ideal pero igual 0 superior al 75\%, las soluciones de conectividad Sigfox, LoRaWAN o LoRa-Sigfox a través del dispositivo propio con función de gateway, permiten alcanzar una tasa de recepción del 100\%. En el caso de ubicaciones pertenecientes al intervalo de entre un $75 \%$ y un $25 \%$ de éxito de datos recibidos, la aplicación de una solución basada en LoRaWAN o a través del gateway LoRa-Sigfox, son las que han demostrado que puede alcanzarse el $100 \%$ en la tasa de recepción. La aplicación de Sigfox también mejora el servicio pero no en la misma medida, quizás por la ubicación concreta de la estación base del operador. En el caso de tasas de recepción actuales inferiores al 25\%, tanto Sigfox y LoRaWAN ofrecen una mejora pero la única solución que permite alcanzar el 100\% es la basada en el dispositivo gateway propio por tratarse de localizaciones especialmente adversas que impiden la suficiente penetración de la señal de las otras redes.

Una mejora frente a soluciones en servicio es que los tres prototipos desarrollados posibilitan alcanzar una tasa de muestreo del consumo acumulado cada 10 minutos. Esta mayor resolución permitiría a la compañía distribuidora tener una mayor cantidad de datos en un periodo de tiempo determinado y por tanto, manejar una información más detallada sobre la que se podrían aplicar técnicas de Big Data con objeto de estimar hábitos de consumo de los usuarios y prever picos de consumo futuros, mejorando las previsiones del servicio así como las estrategias de mantenimiento y ampliación de la red de distribución. A pesar de ello no se debe dejar de considerar el mayor coste energético que esto lleva asociado y que en dispositivos operados por baterías puede suponer encontrar el equilibrio entre prestaciones y coste de mantenimiento.

Se ha preferido un diseño empleando tanto hardware como software abiertos que proporcionen libertad para implementar la funcionalidad requerida y faciliten ampliaciones futuras y soluciones propias tanto a la comunidad científica como a posibles operadores del sistema sin las limitaciones de los productos comerciales cerrados. La implantación masiva de un sistema de telelectura y telecontrol basado en tecnologías genéricas para loT que se muestre eficaz, de coste contenido, personalizable y escalable, permitirían entre otras, evitar la necesidad de tener personal cualificado revisando mensualmente todos los contadores, siendo sólo necesario su actuación en casos puntuales o en situaciones periódicas de revisión y mantenimiento. Esto lo hace más interesante en situaciones en las que las viviendas se encuentran localizadas en accesos remotos tal y como se demuestra en el caso piloto de la zona rural de Campules.

El trabajo realizado en esta tesis queda enmarcado dentro del denominado proyecto Tellot, orientado a la optimización de las infraestructuras hidráulicas de abastecimiento con telelectura de contadores inteligente basa en internet de las cosas (Ref. 2I16SA000044), alineado con la estrategia de investigación e innovación para la especialización Inteligente de la Región de Murcia, RIS3MUR. 
-148 - 
ANEXO 1

INFORMACIÓN ADICIONAL DEL HARDWARE 



\section{A1.1. Dispositivo de contador con Waspmote}

En este apartado se incluye información complementaria que se considera de utilidad para la fabricación del dispositivo de contador que incorpora la placa base Waspmote. Comprende el listado de los componentes necesarios, el diagrama de mecanizado de la carcasa y las etiquetas exteriores.

\section{A1.1.1. Listado de componentes}

El listado de componentes de la parte de control se puede consultar en la Tabla A1.1.

\begin{tabular}{|c|c|c|c|c|c|c|c|}
\hline Ud & Descripción & Fabricante & Ref. Fábrica & Distribuidor & Ref. Distrib & Ud $€$ & Total $€$ \\
\hline 1 & Waspmote Sigfox & Libelium & WSFEULIC & cooking hacks & WSFEULIC & 228 & 228,00 \\
\hline 1 & $\begin{array}{l}\text { Radio LoRaWAN + } \\
\text { Puerto } 2 \text { Expansion } \\
\text { Board }\end{array}$ & Libelium & $\begin{array}{l}\text { LWSMA } \\
\text { 4433EXP }\end{array}$ & cooking hacks & LWSMA4433EXP & 107 & 107,00 \\
\hline 1 & $\begin{array}{l}\text { Antena } 868 \text { para } \\
\text { LoRaWAN }\end{array}$ & Libelium & 10665 & cooking hacks & 10665 & 10 & 10,00 \\
\hline 1 & $\begin{array}{c}\text { Events Sensor } \\
\text { Board }\end{array}$ & Libelium & WEVENTS & cooking hacks & WEVENTS & 90 & 90,00 \\
\hline 1 & $\begin{array}{l}\text { Separadores placa } \\
\text { expansión }\end{array}$ & Libelium & - & cooking hacks & - & 0 & 0,00 \\
\hline 1 & Batería & Libelium & 6041 & cooking hacks & 6041 & 36 & 36,00 \\
\hline 3 & $\begin{array}{l}\text { latiguillo RP-SMA } \\
\text { RP-SMA macho }\end{array}$ & MULTICOMP & MC000792 & Farnell & 2452685 & 11,67 & 35,01 \\
\hline 1 & $\begin{array}{l}\text { Latiguillo USB, Serie } \\
\text { GTC Sealed, } \\
\text { 200mm, Mini a Mini }\end{array}$ & RS-Pro & $111-6755$ & Amidata & $111-6755$ & 20,8 & 20,80 \\
\hline 1 & $\begin{array}{c}\text { Conector panel } 2 \\
\text { pin (electroválvula) }\end{array}$ & RS-Pro & EN3P2F16X & Amidata & 878-7077 & 4,02 & 4,02 \\
\hline 1 & $\begin{array}{c}\text { Conector } \\
\text { enchufable } 2 \text { pin } \\
\text { (electroválvula) }\end{array}$ & RS-Pro & EN3C2M16X & Amidata & 878-6998 & 3,81 & 3,81 \\
\hline 1 & $\begin{array}{c}\text { Conector panel } 2 \\
\text { pin (contador) }\end{array}$ & RS-Pro & EN3P2M16X & Amidata & 878-7073 & 3,24 & 3,24 \\
\hline 1 & $\begin{array}{c}\text { Conector } \\
\text { enchufable } 2 \text { pin } \\
\text { (contador) }\end{array}$ & RS-Pro & EN3C2F16X & Amidata & $878-7023$ & 5,15 & 5,15 \\
\hline 1 & $\begin{array}{c}\text { Caja ABS } \\
\text { Hammond, IP54, } \\
120 \times 120 \times 59 \mathrm{~mm}\end{array}$ & Hammond & 1591UBK & Amidata & $493-6010$ & 9,09 & 9,09 \\
\hline 2 & $\begin{array}{c}\text { Separadores pcb } \\
8 \mathrm{~mm} \mathrm{M} 3 \mathrm{x} \text { M3 }\end{array}$ & RS Pro & $325-722$ & Amidata & $325-722$ & 0,23 & 0,46 \\
\hline 2 & Tornillos M3 x 5mm & RS Pro & $\begin{array}{c}\text { MMS603 } \\
005 C R 3\end{array}$ & Amidata & $908-7668$ & 2,31 & 4,62 \\
\hline 1 & Soporte batería & Dintel & - & - & - & 3 & 3,00 \\
\hline 1 & Pegatinas caja & Dintel & - & - & - & 3 & 3,00 \\
\hline
\end{tabular}

Tabla A1.1. Listado de componentes de la placa de control. 
El listado de componentes de la placa de expansión se encuentra en la Tabla A1.2.

\begin{tabular}{cccccccc}
\hline Ud & Descripción & Fabricante & Ref. Fábrica & Distribuidor & Ref. Distrib & Ud $€$ & Total $€$ \\
\hline 1 & $\begin{array}{c}\text { Relé biestable } \\
\text { bobinas 5V }\end{array}$ & $\begin{array}{c}\text { OEG-TE } \\
\text { Connectivity }\end{array}$ & RT314F05 & Farnell & 2500717 & 6,34 & 6,34 \\
\hline 2 & Transistor BC337 & $\begin{array}{c}\text { ON } \\
\text { Semiconductor }\end{array}$ & BC33725TA & Amidata & $671-1116$ & 0,14 & 0,28 \\
\hline 2 & Resistencia 1 k $\Omega$ & Vishay & $\begin{array}{c}\text { MBB02070C } \\
\text { 1001FCT00 }\end{array}$ & Amidata & $477-7928$ & 0,082 & 0,16 \\
\hline 2 & Resistencia 10 k $\Omega$ & Vishay & $\begin{array}{c}\text { MBB02070C } \\
\text { 1002FCT00 }\end{array}$ & Amidata & $477-8246$ & 0,111 & 0,22 \\
\hline 1 & PCB 100x50mm & C.I.F. & 8903575 & Amidata & $890-3575$ & 2,94 & 2,94 \\
\hline 1 & $\begin{array}{c}\text { Terminal para PCB } \\
\text { C/tornillo 2 vías,5mm }\end{array}$ & $\begin{array}{c}\text { TE } \\
\text { Connectivity }\end{array}$ & 282836-2 & Amidata & $361-7667$ & 0,58 & 0,58 \\
\hline 1 & $\begin{array}{c}\text { Terminal para PCB } \\
\text { C/tornillo 3 vías,5mm }\end{array}$ & $\begin{array}{c}\text { TE } \\
\text { Connectivity }\end{array}$ & 282836-3 & Amidata & $361-7673$ & 0,63 & 0,63 \\
\hline 2 & Diodo 1N4007 & Vishay & 1N4007GP-E3/54 & Amidata & $708-7984$ & 0,13 & 0,26 \\
\hline 1 & Condensador 100 $\mathrm{nF}$ & AVX & SR205C104KAR & Amidata & $537-3707$ & 0,3 & 0,30 \\
\hline & & & & & 11,72
\end{tabular}

Tabla A1.2. Listado de componentes de la placa de expansión.

\section{A1.1.2. Diagrama de mecanizado de la carcasa}

El mecanizado de la carcasa desglosado por caras puede verse en la Figura A1.1, Figura A1.2 y Figura A1.3.
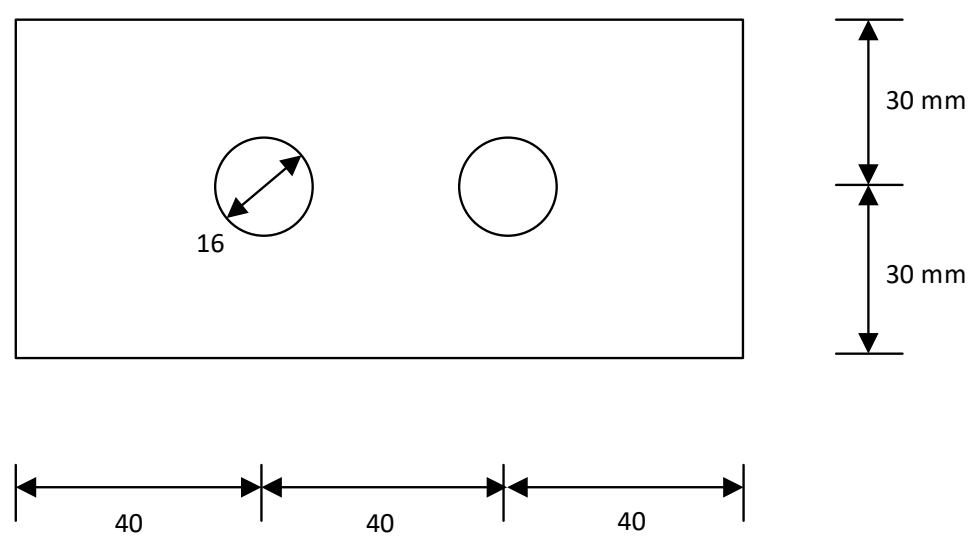

Figura A1.1. Conectores de entrada y salida. Lateral inferior. 


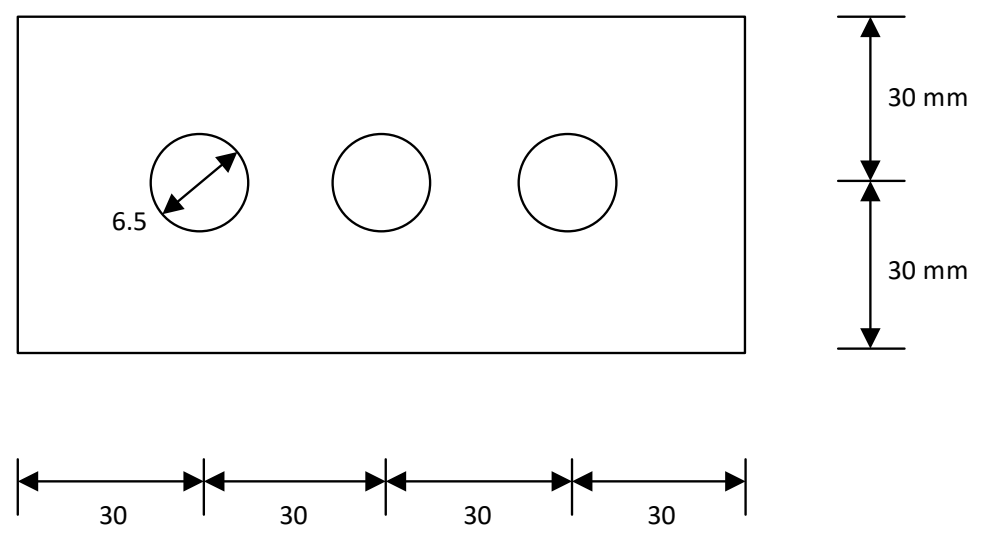

Figura A1.2. Conectores de antena. Lateral superior.
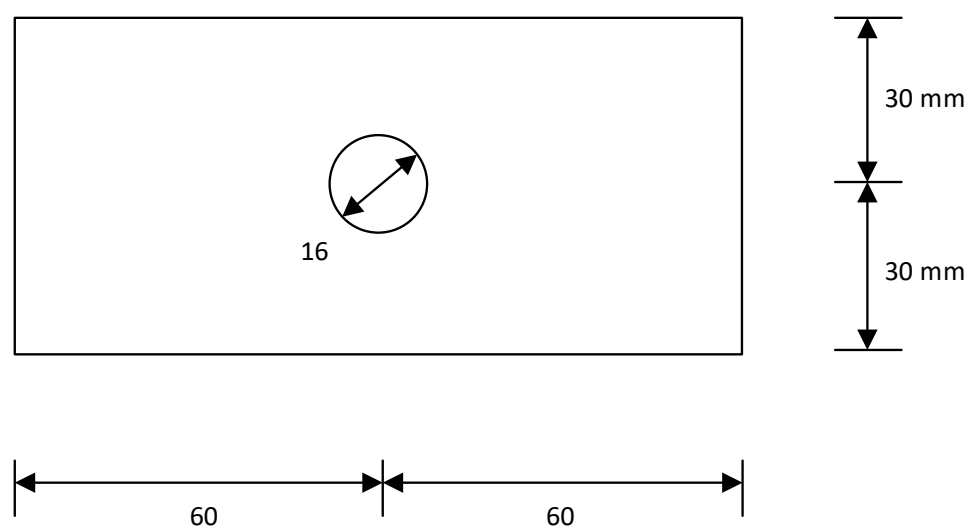

Figura A1.3. Conector USB. Lateral derecho. 


\section{A1.1.3. Etiquetas}

Las etiquetas exteriores de la carcasa son las siguientes: Frontal, de dimensiones $75 \times 75 \mathrm{~mm}$ en la Figura A1.4; conectores RF, de dimensiones 100 x 40 mm en la Figura A1.5 y conexión de panel solar de dimensiones $90 \times 45 \mathrm{~mm}$ en la Figura A1.6.

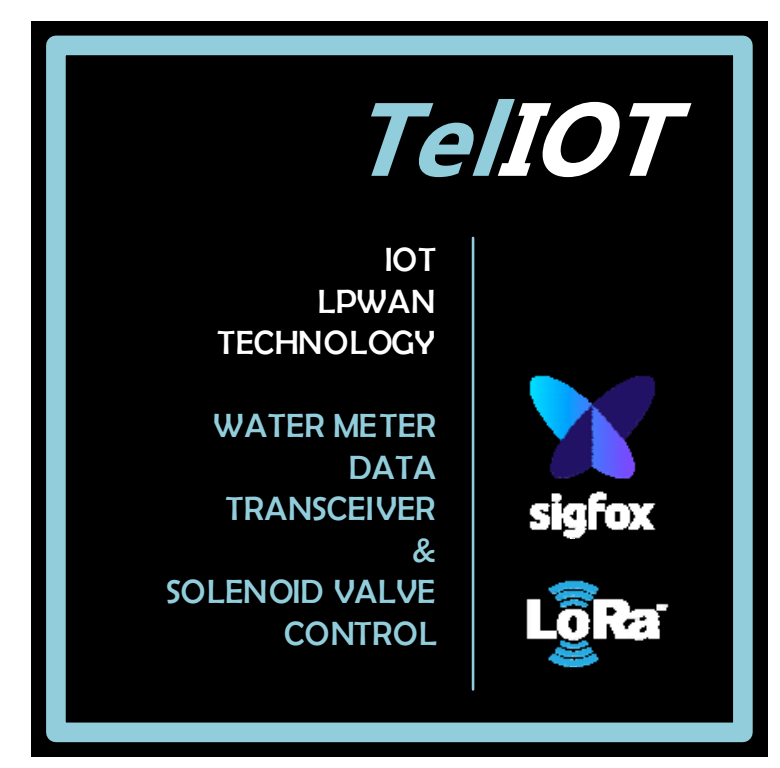

Figura A1.4. Etiqueta de panel frontal.

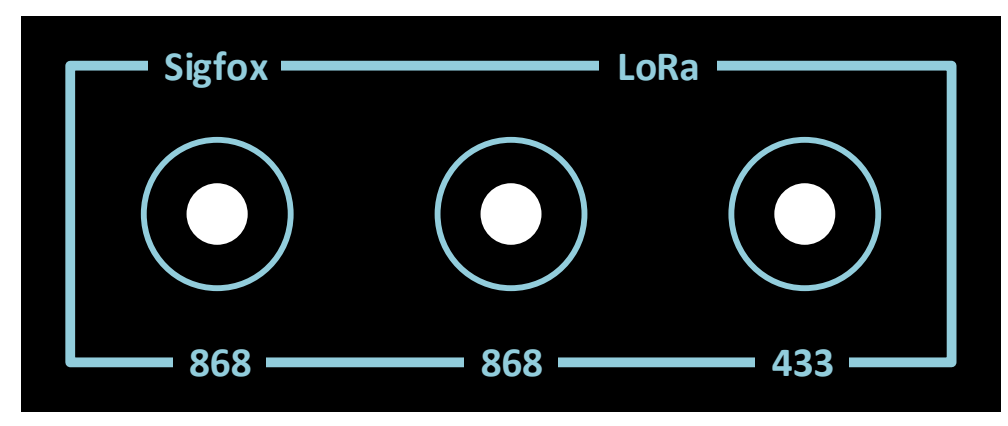

Figura A1.5. Etiqueta de conectores RF.

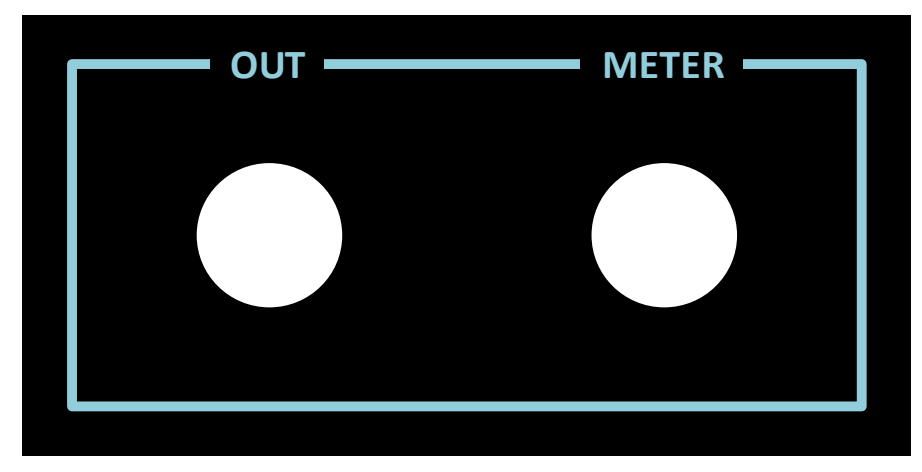

Figura A1.6. Etiqueta de conector de panel solar. 


\section{A1.1.4. Secuencia de montaje}

El montaje ha de realizarse preferentemente en el orden descrito a continuación:

1. Mecanizado de la carcasa plástica:

1.1. Taladros para conectores de entrada y salida: $16 \mathrm{~mm}$, lado inferior

1.2. Taladro para conector USB: $16 \mathrm{~mm}$, lateral derecho

1.3. Taladros para conectores SMA: $6 \mathrm{~mm}$, lado superior

2. Montaje de adhesivos en la caja.

3. Fabricación del cableado entre el conector externo de panel solar la placa principal.

4. Fijación a la caja del conector de panel solar.

5. Conexión de los latiguillos RF RSMA-macho a RSMA-hembra a la placa Waspmote.

6. Montaje de la batería y conexión a la placa base.

7. Fijación de los latiguillos RF a la caja.

8. Montaje de separadores en la placa base y fijación a la caja.

9. Impresión y montaje del separador de batería.

10. Montaje de las antenas externas.

11. Encendido de la placa base (ambos micro-interruptores hacia LEDs).

12. Programación del firmware.

13. Verificación del funcionamiento. 


\section{A1.1.5. Especificaciones técnicas}

\section{Características físicas}

\begin{tabular}{ll}
\hline Dimensiones externas sin antenas & $120 \times 120 \times 59 \mathrm{~mm}$ \\
\hline Peso & $1704 \mathrm{~g}$ \\
\hline Grado de protección IP & -- \\
\hline Material de la carcasa & Aluminio + ABS \\
\hline Temperatura de funcionamiento & $-40^{\circ} \mathrm{C} \mathrm{a}+85^{\circ} \mathrm{C}$ \\
\hline Temperatura de almacenamiento & $-40^{\circ} \mathrm{C} \mathrm{a}+100^{\circ} \mathrm{C}$ \\
\hline
\end{tabular}

\section{Características eléctricas}

\begin{tabular}{ll}
\hline Voltaje de alimentación USB & $5 \mathrm{~V}$ \\
\hline Consumo máximo USB en operación & $500 \mathrm{~mA}$ \\
\hline Tipo de entrada & Cierre de circuito a +3V \\
\hline Voltaje entrada contador & $3 \mathrm{~V}$ \\
\hline Intensidad entrada contador & $43 \mathrm{~mA}$ \\
\hline Tipo de salida & Contacto libre de potencial \\
\hline Intensidad máxima continua a la salida & $1.1 \mathrm{~A}$ \\
\hline Intensidad máxima de pico a la salida & $3.0 \mathrm{~A}$ \\
\hline Tensión de carga de pico en $\mathrm{AC}$ & $60 \mathrm{~V}$ \\
\hline
\end{tabular}

\section{Características radioeléctricas}

\begin{tabular}{ll}
\hline Banda de frecuencias radio & ISM 868 MHz + ISM 434 MHz \\
\hline Rango de frecuencias de transmisión & $868.0-868.6 \mathrm{MHz}$ \\
\hline Rango de frecuencias de recepción & $869.4-869.65 \mathrm{MHz}$ \\
\hline Potencia de emisión & $14 \mathrm{dBm}(25 \mathrm{~mW})$ \\
\hline Ganancia de antena & $4.5 \mathrm{dBm}$ \\
\hline Tecnologías LPWAN soportadas & Sigfox + LoRa \\
\hline Sigfox & Clase 0u \\
\hline Certificación radio & $100 \mathrm{bps}$ \\
\hline Tasa de bits en transmisión & DBPSK \\
\hline Modulación en transmisión & $600 \mathrm{bps}$ \\
\hline Tasa de bits en recepción & GFSK \\
\hline Modulación en recepción & $-126 \mathrm{dBm}$ \\
\hline Sensibilidad de recepción & $52 \mathrm{~mA}$ \\
\hline Consumo en transmisión & $13 \mathrm{~mA}$ \\
\hline Consumo en recepción & $45 \mathrm{~s}$ \\
\hline Duración de transmisión con ack & \\
\hline LoRa & LoRaWAN 1.0 Clase A \\
\hline Certificación radio & $14 \mathrm{dBm}(25 \mathrm{~mW})$ \\
\hline Potencia de emisión & $250-5470 \mathrm{bps}$ \\
\hline Tasa de bits emisión y recepción & $-136 \mathrm{dBm}$ \\
\hline Sensibilidad de recepción & $38.9 \mathrm{~mA}$ \\
\hline Consumo en transmisión & $14.2 \mathrm{~mA}$ \\
\hline Consumo en recepción &
\end{tabular}

Tabla A1.3. Especificaciones técnicas. 


\section{A1.2. Dispositivo de contador con Pycom}

En este apartado se incluye información complementaria que se considera de utilidad para la fabricación del dispositivo de contador con el controlador PyCom. Comprende el listado de componentes necesarios, el diagrama de mecanizado de la carcasa y las etiquetas exteriores.

\section{A1.2.1. Listado de componentes}

El listado de componentes se puede consultar en la Tabla A1.4.

\begin{tabular}{|c|c|c|c|c|c|c|}
\hline Ud & Descripción & Fabricante & Ref. Fábrica & $\begin{array}{l}\text { Ref. } \\
\text { Distrib }\end{array}$ & Ud $€$ & $\begin{array}{l}\text { Total } \\
€\end{array}$ \\
\hline 1 & Pycom LoPy4 & Pycom & LoPy4 RCZ1 & Amidata & $162-8047$ & 46,15 \\
\hline 2 & $\begin{array}{c}\text { Tira de } 20 \text { pines } 1 \text { fila paso } \\
2.54 \mathrm{~mm}\end{array}$ & Winslow & W35520TRC & Amidata & $267-7400$ & 2,92 \\
\hline 1 & $\begin{array}{c}\text { Antena interna ISM-868 con } \\
\text { U.FL }\end{array}$ & Molex & $105262-0002$ & Amidata & $819-7389$ & 3,58 \\
\hline 2 & $\begin{array}{l}\text { Terminal de rosca para } \\
\text { PCB } 2 \text { vías, } 2.54 \mathrm{~mm}\end{array}$ & Phoenix Contact & 1725656 & Amidata & $220-4260$ & 2,84 \\
\hline 1 & $\begin{array}{l}\text { Terminal de rosca para } \\
\text { PCB } 3 \text { vías, } 2.54 \mathrm{~mm}\end{array}$ & Phoenix Contact & 1725669 & Amidata & $220-4276$ & 4,16 \\
\hline 1 & Batería Litio $2300 \mathrm{~mA}$ & LG & LGP523450-01026SZ & Amidata & $826-3470$ & 25,13 \\
\hline 1 & $\begin{array}{l}\text { Conector panel } 2 \text { pin } \\
\text { (electroválvula) }\end{array}$ & RS-Pro & EN3P2F16X & Amidata & $878-7077$ & 3,91 \\
\hline 1 & $\begin{array}{l}\text { Conector enchufable } 2 \text { pin } \\
\text { (electroválvula) }\end{array}$ & RS-Pro & EN3C2M16X & Amidata & $878-6998$ & 3,71 \\
\hline 1 & $\begin{array}{c}\text { Conector panel } 2 \text { pin } \\
\text { (contador) }\end{array}$ & RS-Pro & EN3P2M16X & Amidata & $878-7073$ & 3,15 \\
\hline 1 & $\begin{array}{c}\text { Conector enchufable } 2 \text { pin } \\
\text { (contador) }\end{array}$ & RS-Pro & EN3C2F16X & Amidata & $878-7023$ & 5,02 \\
\hline 1 & $\begin{array}{c}\text { Caja ABS Hammond } \\
\text { 1594CBK }\end{array}$ & Hammond & 1594CBK & Amidata & $528-6978$ & 9,92 \\
\hline 4 & $\begin{array}{l}\text { Separadores pcb 8mm M3 x } \\
\text { M3 }\end{array}$ & RS Pro & $325-722$ & Amidata & $325-722$ & 12,85 \\
\hline 4 & Tornillos M3 x 5mm & RS Pro & MMS603 005CR3 & Amidata & $908-7668$ & 2,37 \\
\hline 2 & Transistor PDTD113ZT & Nexperia & PDTD113ZT,215 & Amidata & $508-541$ & 3,30 \\
\hline 1 & Resistencia $1 \mathrm{k} \Omega 0805$ & TE Connectivity & CRG0805F1K0 & Amidata & $223-0427$ & 0,85 \\
\hline 1 & Resistencia $22 \mathrm{k} \Omega 0805$ & TE Connectivity & CRG0805F22K & Amidata & $223-0607$ & 1,30 \\
\hline 1 & Resistencia $150 \mathrm{k} \Omega 0805$ & TE Connectivity & CRG0805F150K & Amidata & $223-0714$ & 1,25 \\
\hline 1 & Resistencia $47 \mathrm{k} \Omega 0805$ & TE Connectivity & CRG0805F47K & Amidata & $223-0641$ & 1,90 \\
\hline 2 & Diodo MBRA140T3G & ON Semi & MBRA140T3G & Amidata & $625-5650$ & 1,52 \\
\hline 1 & Condensador 100 nF 0805 & KEMET & C0805C104J5RACTU & Amidata & $648-0941$ & 0,30 \\
\hline 1 & Puente & RS Pro & $251-8575$ & Amidata & $251-8575$ & 3,91 \\
\hline 1 & Pines para puente & Molex & $42375-0002$ & Amidata & $896-7620$ & 2,70 \\
\hline
\end{tabular}

Tabla A1.4. Listado de componentes. 


\section{A1.2.2. Diagrama de mecanizado de la carcasa}

El mecanizado de la carcasa se encuentra detallado en las siguientes figuras: Figura A1.7: Conector de entrada de pulsos (Lateral izquierdo) y Figura A1.8: Conector de salida para electroválvula (Lateral derecho).
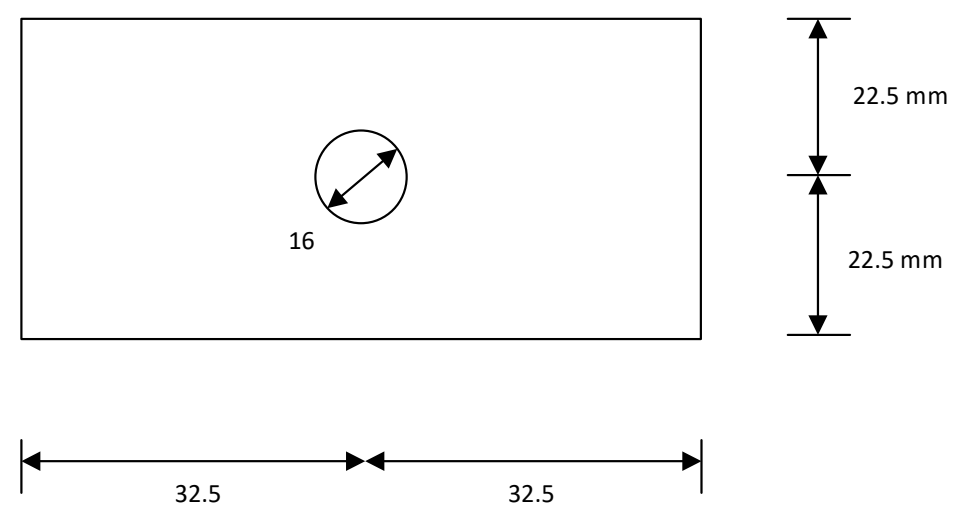

Figura A1.7. Conector de entrada de pulsos. Lateral izquierdo.

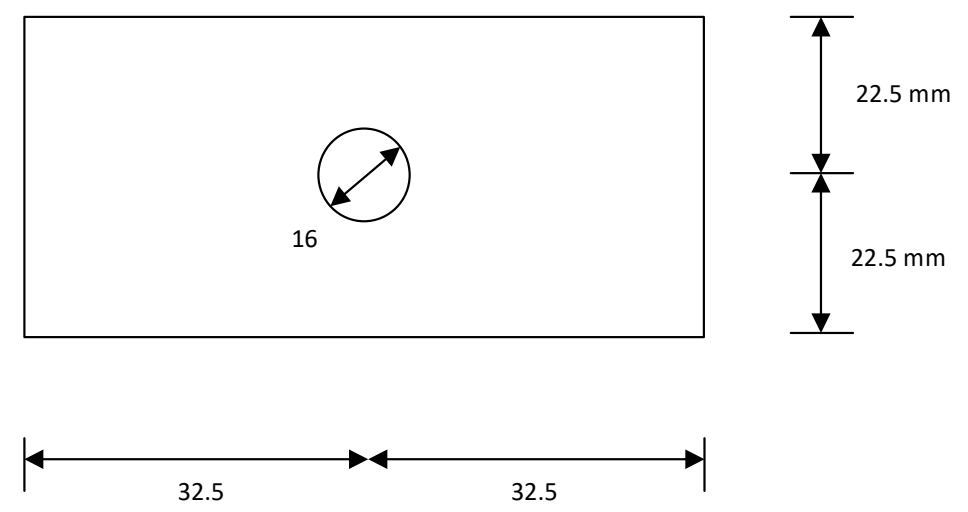

Figura A1.8. Conector de salida para electroválvula. Lateral derecho. 


\section{A1.2.3. Etiquetas}

La etiqueta de la carcasa se puede ver en la Figura A1.9. Sus dimensiones son de $75 \times 75 \mathrm{~mm}$.

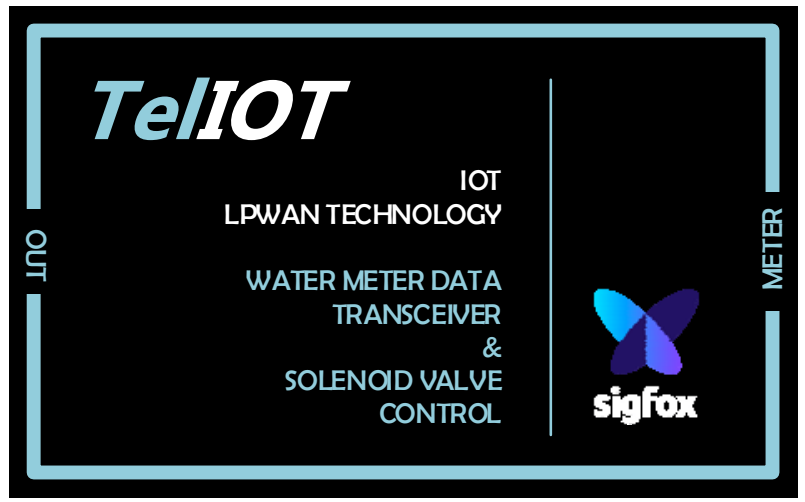

Figura A1.9. Etiqueta en la cara superior de la carcasa.

\section{A1.2.4. Secuencia de montaje}

El montaje se recomienda realizarse preferentemente en el orden descrito a continuación:

1. Programación del módulo Pycom en placa de expansión.

2. Mecanizado de la caja. Taladros de $16 \mathrm{~mm}$ a ambos lados para conectores.

3. Pegar antena interior en la cara mayor.

4. Soldar cableado a conectores.

5. Montaje de conectores.

6. Fijar la batería con adhesivo.

7. Montaje de separadores en la placa base y fijación a la caja con adhesivo.

8. Conexionado del cable de antena al módulo Pycom.

9. Conexionado a la placa del cableado de los conectores externos

10. Quitar puente de alimentación de la placa base.

11. Conexionado del cableado de la batería.

Para la puesta en marcha del dispositivo se han de llevar a cabo las siguientes acciones:

1. Conexión de la señal de contador al conector de entrada.

2. Conexión del circuito a controlar al conector de salida.

3. Poner puente de activación y verificar encendido del LED.

4. Verificación de la trama transmitida en el servidor.

5. Verificación de la operación periódica.

6. Verificación de actuación de la salida modificando el parámetro en el servidor.

7. Verificación de lectura de pulsos en la entrada según valor periódico enviado. 


\section{A1.2.5. Especificaciones técnicas}

\section{Características físicas}

\begin{tabular}{ll}
\hline Dimensiones externas & $105 \times 65 \times 45 \mathrm{~mm}$ \\
\hline Peso & $210 \mathrm{~g}$ \\
\hline Grado de protección IP & IP54 \\
\hline Material de la carcasa & ABS pirorretardante conforme a UL94V-0 \\
\hline Rango de temperatura & $-10^{\circ} \mathrm{C} \mathrm{a}+85^{\circ} \mathrm{C}$ \\
\hline
\end{tabular}

\section{Características eléctricas}

\begin{tabular}{ll}
\hline Voltaje de operación & $3.5-5.5 \mathrm{~V}$ \\
\hline Consumo en operación & $37.7 \mathrm{~mA}$ \\
\hline Consumo en hibernación & $58 \mathrm{uA}$ \\
\hline Consumo utilizando radio LPWAN & $157 \mathrm{~mA}$ \\
\hline Batería tecnología & Polímero de lon-Litio \\
\hline Batería características eléctricas & $3.7 \mathrm{~V} / 2300 \mathrm{~mA}$ \\
\hline Batería rango de voltaje & $2.6-4.2 \mathrm{~V}$ \\
\hline Batería máximas tensión y corriente de carga & $4.2 \mathrm{~V} / 1150 \mathrm{~mA}$ \\
\hline Microcontrolador & Espressif ESP32 \\
\hline Memoria SRAM & $520 \mathrm{kB}$ \\
\hline Memoria Flash & $1 \mathrm{MB}$ \\
\hline
\end{tabular}

\section{Características radioeléctricas}

\begin{tabular}{ll}
\hline Banda de frecuencias radio & ISM $868 \mathrm{MHz}$ \\
\hline Rango de frecuencias de transmisión & $868.130-869.525 \mathrm{MHz}$ \\
\hline Potencia de emisión & $14 \mathrm{dBm}(25 \mathrm{~mW})$ \\
\hline Ganancia de antena & $4.5 \mathrm{dBm}$ \\
\hline Tecnologías LPWAN soportadas & Sigfox / LoRa \\
\hline Sigfox & \\
\hline Certificación radio & Clase 0u \\
\hline Tasa de bits en transmisión & $100 \mathrm{bps}$ \\
\hline Modulación en transmisión & DBPSK \\
\hline Tasa de bits en recepción & $600 \mathrm{bps}$ \\
\hline Modulación en recepción & GFSK \\
\hline Sensibilidad de recepción & $-126 \mathrm{dBm}$ \\
\hline Consumo en transmisión & $47 \mathrm{~mA}$ \\
\hline Consumo en recepción & $17 \mathrm{~mA}$ \\
\hline Duración de transmisión con ack & $45 \mathrm{~s}$ \\
\hline LoRa & \\
\hline Certificación radio & LoRaWAN 1.0 Clase A \\
\hline Potencia de emisión & $14 \mathrm{dBm}(25 \mathrm{~mW})$ \\
\hline Tasa de bits emisión y recepción & $250-5470 \mathrm{bps}$ \\
\hline Sensibilidad de recepción & $-136 \mathrm{dBm}$ \\
\hline Consumo en transmisión & $38.9 \mathrm{~mA}$ \\
\hline Consumo en recepción & $14.2 \mathrm{~mA}$ \\
\hline
\end{tabular}

Tabla A1.5. Especificaciones técnicas. 


\section{A1.3. Gateway loT LoRa-Sigfox}

A continuación se incluye información complementaria que se considera de utilidad para la fabricación del dispositivo gateway loT LoRa-Sigfox.

\section{A1.3.1. Listado de componentes}

El listado de componentes es el reflejado en la siguiente Tabla A1.6.

\begin{tabular}{|c|c|c|c|c|c|c|c|}
\hline Ud & Descripción & Fabricante & Ref. Fábrica & Distribuidor & Ref. Distrib & Ud $€$ & Total $€$ \\
\hline 1 & $\begin{array}{lll}\text { Waspmote } & \text { Sigfox } & \text { con } \\
\text { antena }\end{array}$ & Libelium & WSFEULIC & cooking hacks & WSFEULIC & 228 & 228,00 \\
\hline 1 & $\begin{array}{l}\text { Radio LoRaWAN ron } \\
\text { antena + Expansion Board } \\
\text { puerto } 2\end{array}$ & Libelium & $\begin{array}{l}\text { LWSMA } \\
\text { 4433EXP }\end{array}$ & cooking hacks & $\begin{array}{l}\text { LWSMA4433 } \\
\text { EXP }\end{array}$ & 107 & 107,00 \\
\hline 1 & Antena 868 para LoRaWAN & Libelium & 10665 & cooking hacks & 10665 & 10 & 10,00 \\
\hline 1 & Events Sensor Board & Libelium & WEVENTS & cooking hacks & WEVENTS & 90 & 90,00 \\
\hline 1 & $\begin{array}{ll}\text { Separadores } & \text { placa } \\
\text { expansión }\end{array}$ & Libelium & - & cooking hacks & - & 0 & 0,00 \\
\hline 1 & Batería Li-lon 3V7 6600mA & Libelium & 6041 & cooking hacks & 6041 & 36 & 36,00 \\
\hline 3 & $\begin{array}{lr}\text { Latiguillo } & \text { RP-SMA } \\
\text { pasamuros } & \text { RP-SMA macho }\end{array}$ & Multicomp & MC000792 & Farnell & 2452685 & 11,67 & 35,01 \\
\hline 1 & $\begin{array}{l}\text { Latiguillo USB, Serie GTC } \\
\text { Sealed, 200mm, Mini a Mini }\end{array}$ & RS-Pro & - & RS-Amidata & $111-6755$ & 20,8 & 20,80 \\
\hline 1 & Conector panel 2 pin (solar) & RS-Pro & EN3P2M16X & RS-Amidata & 878-7073 & 3,24 & 3,24 \\
\hline 1 & $\begin{array}{l}\text { Conector enchufable } 2 \text { pin } \\
\text { (solar) }\end{array}$ & RS-Pro & EN3C2F16X & RS-Amidata & $878-7023$ & 5,15 & 5,15 \\
\hline 1 & $\begin{array}{ll}\text { Caja ABS } & \text { Hammond } \\
1591 \text { UBK, 1591, IP54, } 120 \\
\text { x } 120 \times 59 \mathrm{~mm}\end{array}$ & Hammond & 1591UBK & RS-Amidata & $493-6010$ & 9,09 & 9,09 \\
\hline 2 & $\begin{array}{l}\text { Separadores PCB 8mm M3 } \\
\text { x M3 }\end{array}$ & RS Pro & $325-722$ & RS-Amidata & $325-722$ & 0,23 & 0,46 \\
\hline 2 & Tornillos M3 x 5mm & RS Pro & $\begin{array}{l}\text { MMS603 } \\
005 C R 3 \\
\end{array}$ & RS-Amidata & $908-7668$ & 2,31 & 4,62 \\
\hline 1 & Soporte batería & Dintel & - & - & - & 3,00 & 3,00 \\
\hline 1 & Juego de pegatinas caja & Dintel & - & - & - & 2,00 & 2,00 \\
\hline 1 & Panel solar & BP Solar & MSX-01F & RS-Amidata & $194-133$ & 49,17 & 49,17 \\
\hline
\end{tabular}

Tabla A1.6. Listado de componentes. 


\section{A1.3.2. Diagrama de mecanizado de la carcasa}
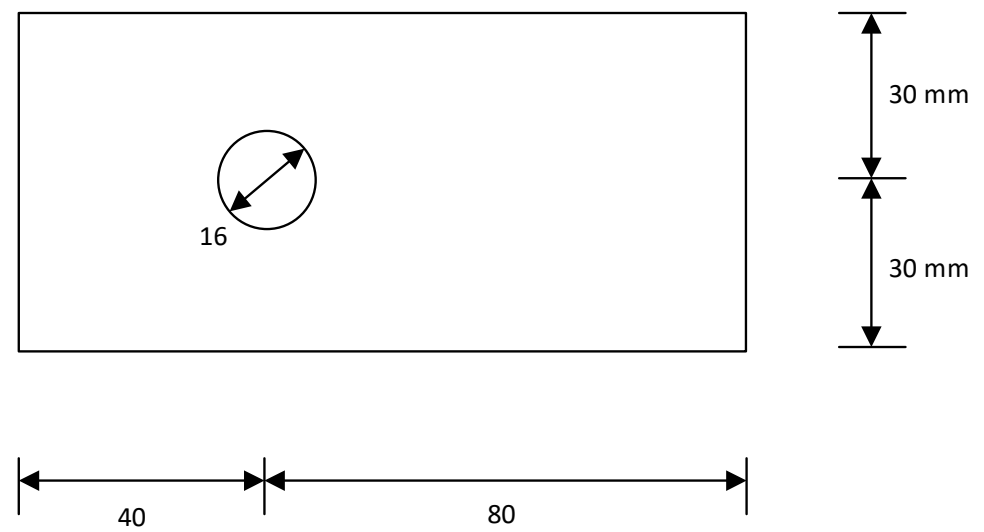

Figura A1.10. Conector de panel solar. Lateral inferior.
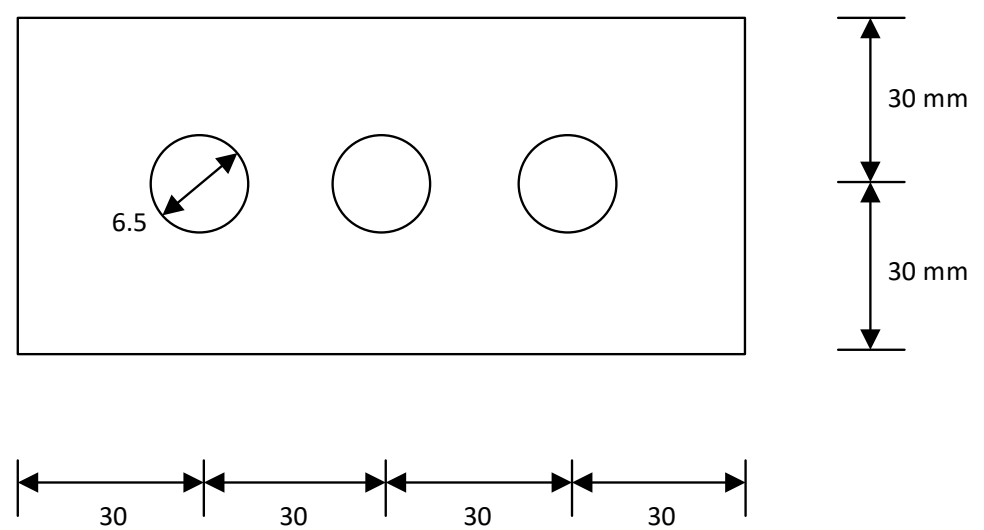

Figura A1.11. Conectores de antena. Lateral superior.
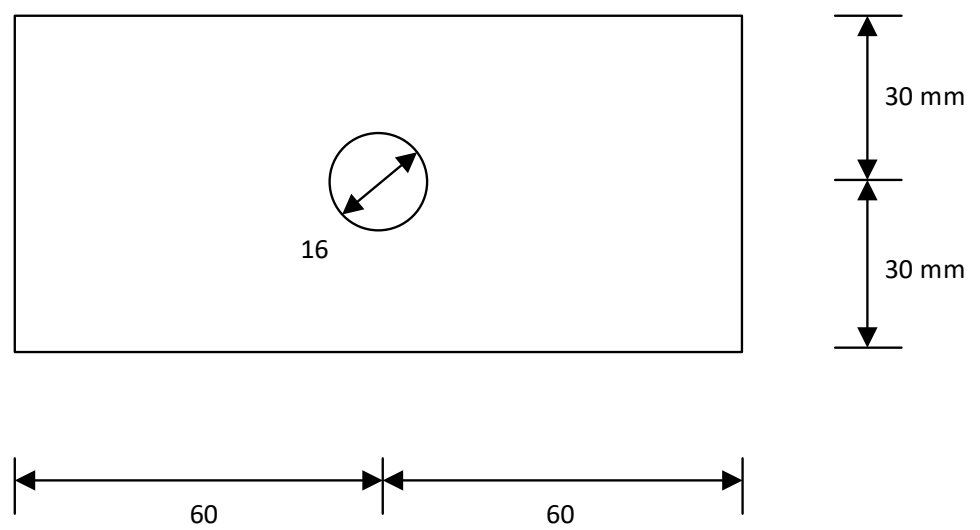

Figura A1.12. Conector USB. Lateral derecho. 


\section{A1.3.3. Etiquetas}

Las etiquetas exteriores de la carcasa son las siguientes: Frontal de dimensiones $75 \times 75 \mathrm{~mm}$ en la Figura A1.13, conectores RF de dimensiones 100 x 40 mm en la Figura A1.14 y conexión de panel solar de dimensiones $90 \times 45 \mathrm{~mm}$ en la Figura A1.15.

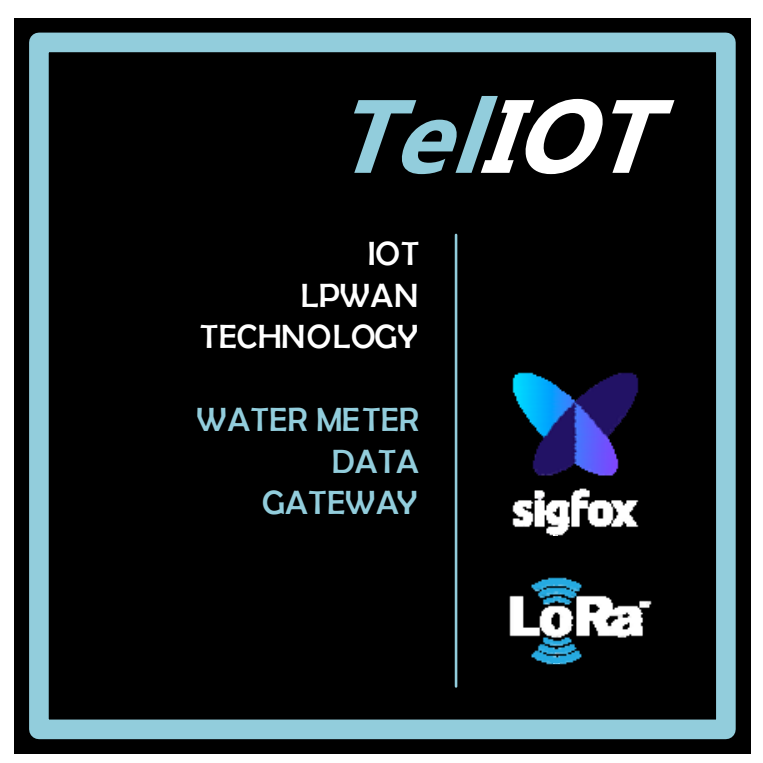

Figura A1.13. Etiqueta de panel frontal.

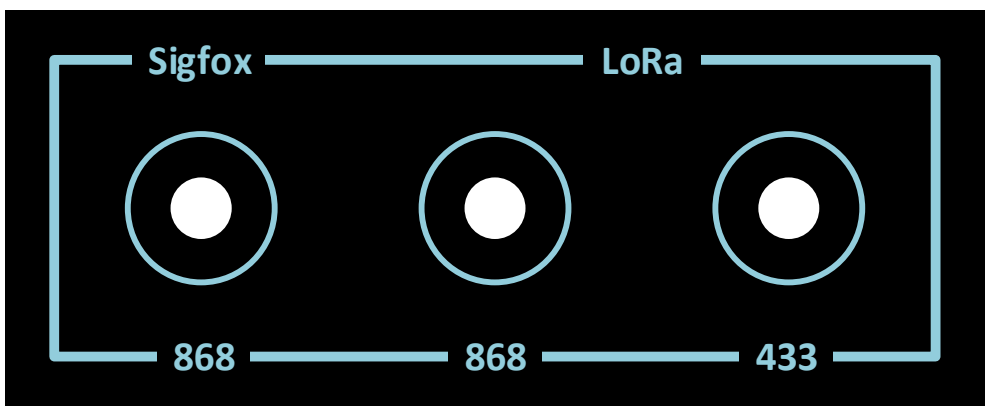

Figura A1.14. Etiqueta de conectores RF.

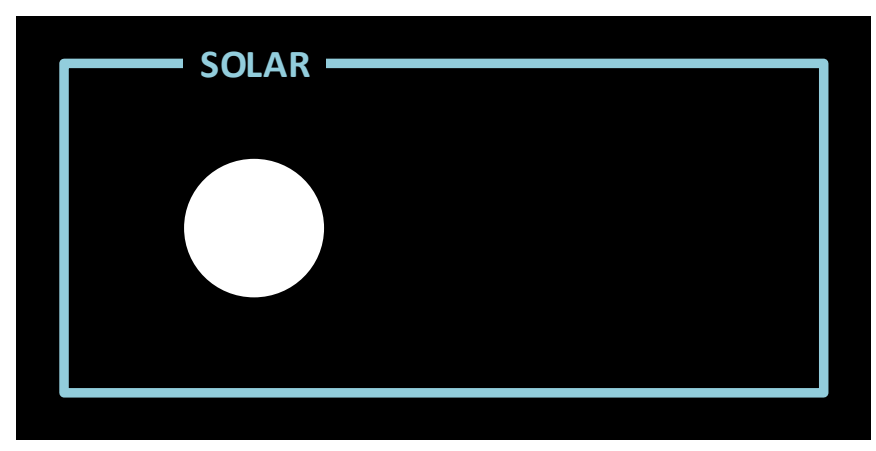

Figura A1.15. Etiqueta de conector de panel solar. 


\section{A1.3.4. Secuencia de montaje}

La secuencia de montaje ha de realizarse preferentemente en el orden descrito a continuación:

1. Mecanizado de la carcasa plástica:

1.1. Taladros para conector de panel solar: $16 \mathrm{~mm}$, lado inferior

1.2. Taladro para conector USB: $16 \mathrm{~mm}$, lateral derecho.

1.3. Taladros para conectores SMA: $6 \mathrm{~mm}$, lado superior.

2. Montaje de adhesivos en la caja.

3. Fabricación del cableado entre el conector externo de panel solar la placa principal.

4. Fijación a la caja del conector de panel solar.

5. Conexión de los latiguillos RF RSMA-macho a RSMA-hembra a la placa Waspmote.

6. Montaje de la batería y conexión a la placa base.

7. Fijación de los latiguillos RF a la caja.

8. Montaje de separadores en la placa base y fijación a la caja.

9. Impresión y montaje del separador de batería.

10. Montaje de las antenas externas.

11. Encendido de la placa base (ambos micro-interruptores hacia LEDs).

12. Programación del firmware.

13. Verificación del funcionamiento. 


\section{A1.3.5. Especificaciones técnicas}

\section{Características físicas}

Dimensiones externas sin antenas $120 \times 120 \times 59 \mathrm{~mm}$

Peso $457 \mathrm{~g}$

Grado de protección IP IP54

Material de la carcasa ABS

Rango de temperatura $-10{ }^{\circ} \mathrm{Ca}+65^{\circ} \mathrm{C}$

\section{Características eléctricas}

Voltaje de operación

$3.3 \mathrm{~V}$

Consumo en espera

$9 \mathrm{~mA}$

Voltaje entrada USB

$5 \mathrm{~V}$

Máxima corriente USB

$100 \mathrm{~mA}$

Voltaje entrada panel solar

$6-12 \mathrm{~V}$

Máxima corriente de panel solar

$240 \mathrm{~mA}$

Batería interna tecnología

Polímero de lón-Litio

Batería interna características eléctricas

$3.7 \mathrm{~V} / 6600 \mathrm{~mA}$

Tiempo mínimo de recarga 0-100\%

Microcontrolador

Frecuencia de operación

Memoria SRAM

66 horas USB / 24 horas solar

ATmega1281

Memoria Flash

$14.74 \mathrm{MHz}$

Memoria SD

$8 \mathrm{~KB}$

$128 \mathrm{kB}$

$2 \mathrm{~GB}$

\section{Características radioeléctricas}

Banda de frecuencias radio

ISM $868 \mathrm{MHz}+$ ISM $434 \mathrm{MHz}$

\begin{tabular}{ll}
\hline Rango de frecuencias de transmisión & $868.0-868.6 \mathrm{MHz}$ \\
\hline Rango de frecuencias de recepción & $869.4-869.65 \mathrm{MHz}$ \\
\hline Potencia de emisión & $14 \mathrm{dBm}(25 \mathrm{~mW})$ \\
\hline Ganancia de antena & $4.5 \mathrm{dBm}$ \\
\hline Sigfox & Clase 0u \\
\hline Certificación radio & $100 \mathrm{bps}$ \\
\hline Tasa de bits en transmisión & DBPSK \\
\hline Modulación en transmisión & $600 \mathrm{bps}$ \\
\hline Tasa de bits en recepción & GFSK \\
\hline Modulación en recepción & $-126 \mathrm{dBm}$ \\
\hline Sensibilidad de recepción & $52 \mathrm{~mA}$ \\
\hline Consumo en transmisión & $13 \mathrm{~mA}$ \\
\hline Consumo en recepción & $45 \mathrm{~s}$ \\
\hline Duración de transmisión con ack & \\
\hline LoRa & LoRaWAN 1.0 Clase A \\
\hline Certificación radio & $14 \mathrm{dBm}(25 \mathrm{~mW})$ \\
\hline Potencia de emisión & $250-5470 \mathrm{bps}$ \\
\hline Tasa de bits emisión y recepción & $-136 \mathrm{dBm}$ \\
\hline Sensibilidad de recepción & $38.9 \mathrm{~mA}$ \\
\hline Consumo en transmisión & $14.2 \mathrm{~mA}$
\end{tabular}

Tabla A1.7. Especificaciones técnicas. 


\section{A1.4. Estación base LoRaWAN}

A continuación se incluye información complementaria que se considera de utilidad para la fabricación de la estación base LoRaWAN.

\section{A1.4.1. Listado de componentes}

El listado de componentes es el reflejado en la siguiente Tabla A1.8.

\begin{tabular}{|c|c|c|c|c|c|c|c|}
\hline Ud & Descripción & Fabricante & Ref. Fábrica & Distribuidor & Ref. Distrib & $\mathrm{Ud} €$ & Total $€$ \\
\hline 1 & Caja policarbonato & Spelsberg & 20041201 & Amidata & $772-1091$ & 29.46 & 29.46 \\
\hline 1 & $\begin{array}{ll}\text { Panel } & \text { interior } \\
220 \times 152 \times 2.5 & \\
\end{array}$ & Spelsberg & 18601201 & Amidata & 7721149 & 7.32 & 7.32 \\
\hline 1 & Kit montaje mástil & Rolec & 501.100 .000 & Amidata & $819-4037$ & 15.15 & 15.15 \\
\hline 1 & $\begin{array}{l}\text { Latiguillo SMA H-Inv a M- } \\
\text { Inv }\end{array}$ & Multicomp & MC000792 & Farnell & 2452685 & 24.76 & 24.76 \\
\hline 1 & Raspberry PI 3 model B & Raspberry & $\mathrm{PI} 3$ & Amidata & 8968660 & 30.19 & 30.19 \\
\hline 1 & iC880A-SPI LoRaWAN & IMST & - & IMST & - & 119 & 119 \\
\hline 1 & Pigtail para iC880A & IMST & - & IMST & - & 6.5 & 6.5 \\
\hline 1 & Antena SMA & IMST & - & IMST & - & 6.5 & 6.5 \\
\hline 1 & $\begin{array}{l}\text { Placa backplane para } \\
\text { ic880A }\end{array}$ & gnz & - & Tindie & - & 5 & 5 \\
\hline 1 & Módulo Sigfox XBee & Libelium & - & $\begin{array}{l}\text { Cooking- } \\
\text { Hacks }\end{array}$ & - & 66 & 66 \\
\hline 1 & Latiguillo red $300 \mathrm{~mm}$ & $\begin{array}{l}\text { Hellermann } \\
\text { Tyton }\end{array}$ & PCBLU1 & Amidata & 8323430 & 1.07 & 1.07 \\
\hline 1 & $\begin{array}{l}\text { Cables macho a hembra } \\
10 \mathrm{Ud}\end{array}$ & $\begin{array}{l}\text { MikroElektro } \\
\text { nika }\end{array}$ & MIKROE-512 & Amidata & 7916454 & 2.41 & 2.41 \\
\hline 1 & $\begin{array}{ll}\text { Bolsa } & \text { separadores } \\
\text { M3x8mm } & \\
\end{array}$ & RS Pro & $325-722$ & Amidata & $325-722$ & 0.236 & 0.236 \\
\hline 1 & Tornillos M3x5mm & RS Pro & $908-7668$ & Amidata & $908-7668$ & 2.37 & 2.37 \\
\hline 1 & $\begin{array}{l}\text { Tarjeta MicroSDHC } 32 \\
\text { GB }\end{array}$ & Samsung & $\begin{array}{c}\text { MB- } \\
\text { MC32GA/EU }\end{array}$ & Amidata & 1449017 & 15.61 & 15.61 \\
\hline 1 & Conector panel RJ45 & Bulgin & PX0833 & Amidata & 4686232 & 31.25 & 31.25 \\
\hline 1 & Tapa conector panel & Bulgin & PX0733 & Amidata & 5044588 & 1.16 & 1.16 \\
\hline 1 & PoE Splitter & DSLRKIT & - & Amazon & DSLRKIT & 9.5 & 9.5 \\
\hline 1 & Inyector PoE & Ubiquiti & $\begin{array}{l}\text { POE-48- } \\
24 W-G\end{array}$ & Amazon & $\begin{array}{l}\text { POE-48- } \\
24 W-G\end{array}$ & 17.73 & 17.73 \\
\hline 1 & $\begin{array}{l}\text { Placa de matriz paso } \\
2 \times 2 \mathrm{~mm}\end{array}$ & $\begin{array}{c}\text { Roth } \\
\text { Elektronik }\end{array}$ & RE130-LF & Amidata & $516-7568$ & 5.17 & 5.17 \\
\hline
\end{tabular}

Tabla A1.8. Listado de componentes. 


\section{A1.4.2. Etiqueta}

En la Figura A1.16 puede verse la etiqueta utilizada en la carcasa de la estación base LORaWAN.

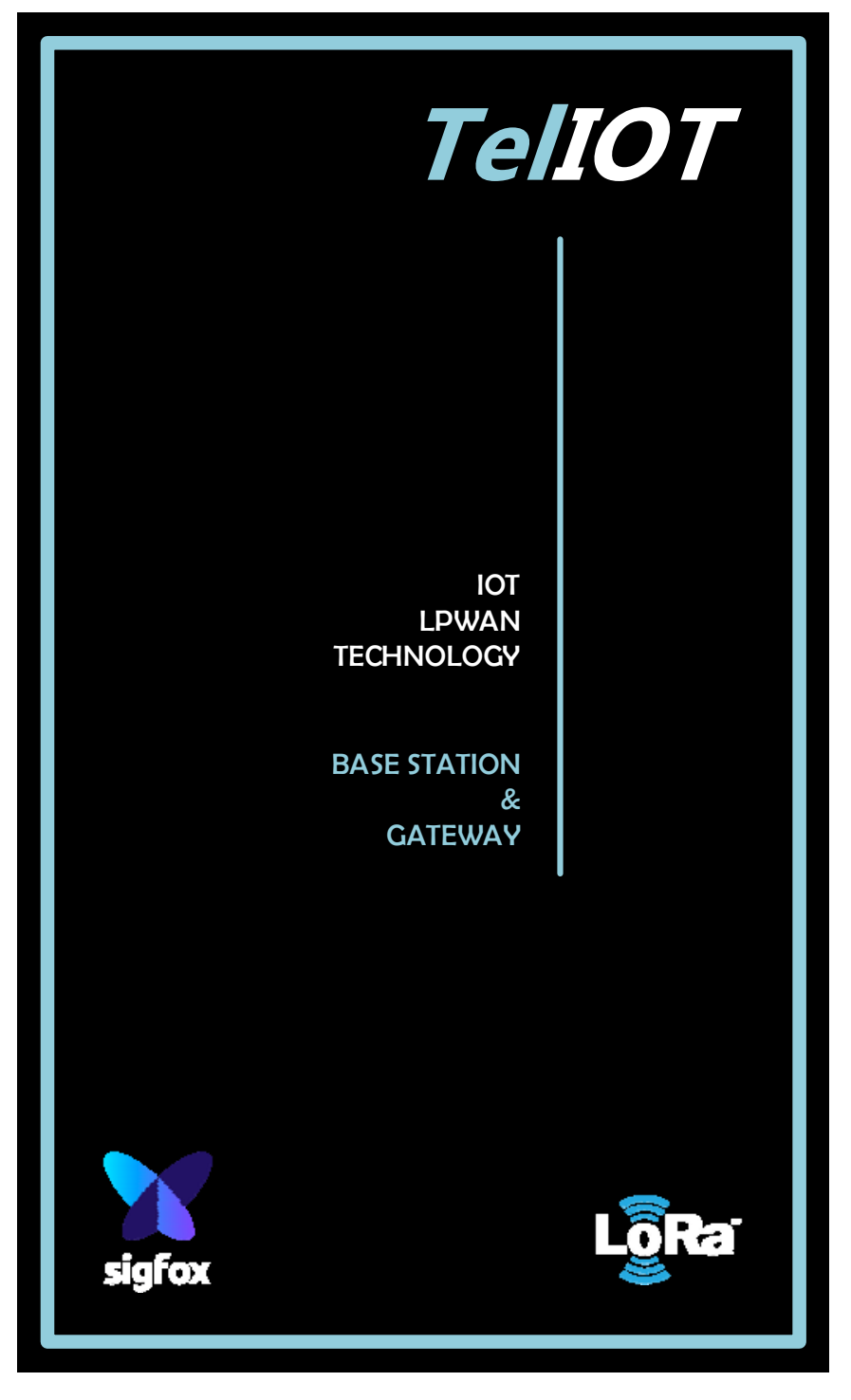

Figura A1.16. Etiqueta de la carcasa de la estación base LoRaWAN. 


\section{A1.5. Consola de pruebas}

A continuación se incluye información complementaria referente al hardware de la consola de pruebas.

\section{A1.5.1. Listado de componentes}

El listado de los elementos que componen la consola es el presente en la Tabla A1.9.

\begin{tabular}{|c|c|c|c|c|c|c|c|}
\hline Ud & Descripción & Fabricante & Referencia & Distribuidor & Referencia & $U d €$ & Total $€$ \\
\hline 1 & Bastidor & Bopla & 92865300MT1 & Amidata & $704-3944$ & 47,61 & 47,61 \\
\hline 1 & $\begin{array}{c}\text { Juego patas de } \\
\text { goma }\end{array}$ & Bopla & 84006700 & Amidata & 704-3978 & 2,17 & 2,17 \\
\hline 1 & Carril DIN & RS Pro & 188-2207 & Amidata & $188-2207$ & 3,79 & 3,79 \\
\hline 1 & Bolsa pasamuros & SES & 01070017010 & Amidata & $136-6935$ & 5,41 & 5,41 \\
\hline 1 & Conmutador & $C \& K$ & $\begin{array}{c}\text { 7201J91ZQ } \\
\text { E22 } \\
\end{array}$ & Amidata & 283-8992 & 7,31 & 7,31 \\
\hline 1 & $\begin{array}{c}\text { Conector panel } \\
\text { macho } 2 \text { pin }\end{array}$ & Switchcraft & EN3P2M16X & Amidata & 878-7073 & 3,24 & 3,24 \\
\hline \multirow[t]{2}{*}{3} & $\begin{array}{l}\text { Conector panel } \\
\text { hembra } 2 \text { pin }\end{array}$ & Switchcraft & EN3P2F16X & Amidata & 878-7077 & 4,02 & 4,02 \\
\hline & $\begin{array}{l}\text { Conector panel } \\
\text { micro-USB }\end{array}$ & RS Pro & $111-6751$ & Amidata & $111-6751$ & 17,57 & 17,57 \\
\hline 1 & $\begin{array}{c}\text { Replicador de } \\
\text { pulsos }\end{array}$ & TelloT & - & - & - & 59,55 & 59,55 \\
\hline 1 & $\begin{array}{c}\text { Emulador de } \\
\text { contador }\end{array}$ & TelloT & - & - & - & 77,16 & 77,16 \\
\hline 1 & $\begin{array}{c}\text { Transceptor pulsos } \\
\text { Sigfox } \\
\end{array}$ & AdeunisRF & ARF8048PA & Matrix & ARF8048PA & 89 & 89 \\
\hline 1 & $\begin{array}{c}\text { Transceptor pulsos } \\
\text { LoRaWAN } \\
\end{array}$ & AdeunisRF & ARF8046PA & Matrix & ARF8046PA & 89 & 89 \\
\hline 1 & $\begin{array}{c}\text { Transceptor pulsos } \\
\text { WmBus }\end{array}$ & AdeunisRF & ARF8043AA & Matrix & ARF8043AA & 79 & 79 \\
\hline 1 & $\begin{array}{c}\text { Transceptor } \\
\text { sensores Sigfox } \\
\end{array}$ & AdeunisRF & ARF8047PA & Matrix & ARF8047PA & 99 & 99 \\
\hline 1 & $\begin{array}{c}\text { Cableado fino de } \\
\text { color }\end{array}$ & - & - & Rayte & - & 0,5 & 0,5 \\
\hline
\end{tabular}

Tabla A1.9. Listado de componentes.

Listado de los conectores a usar en el cable que enlaza los dispositivos externos a la consola, se indica en la Tabla A1.10

\begin{tabular}{clllcccc}
\hline Ud & Descripción & Fabricante & Referencia & Distribuidor & Referencia & $€$ Ud & $€$ \\
\hline 1 & Entrada de contador & Switchcraft & EN3C2F16X & Amidata & $878-7023$ & 5,15 & 5,15 \\
\hline 1 & Salidas a dispositivos & Switchcraft & EN3C2M16X & Amidata & $878-6998$ & 3,81 & 3,81 \\
\hline
\end{tabular}

Tabla A1.10. Listado de componentes. 


\section{A1.5.2. Especificaciones técnicas}

\section{Características físicas}

Dimensiones externas sin antenas

Peso

Grado de protección IP

Material de la carcasa

Temperatura de funcionamiento

Temperatura de almacenamiento
$120 \times 120 \times 59 \mathrm{~mm}$

$1704 \mathrm{~g}$

$--$

Aluminio + ABS

$-40^{\circ} \mathrm{C} \mathrm{a}+85^{\circ} \mathrm{C}$

$-40^{\circ} \mathrm{C} \mathrm{a}+100^{\circ} \mathrm{C}$

\section{Características eléctricas}

Voltaje de alimentación USB

$5 \mathrm{~V}$

Consumo máximo USB en operación

$500 \mathrm{~mA}$

Tipo de entrada

Voltaje entrada contador Cierre de circuito a $+3 \mathrm{~V}$

Intensidad entrada contador $3 \mathrm{~V}$

Tipo de salida

Intensidad máxima continua a la salida

Intensidad máxima de pico a la salida

Tensión de carga de pico en AC

$43 \mathrm{~mA}$

Contacto libre de potencial

$1.1 \mathrm{~A}$

$3.0 \mathrm{~A}$

$60 \mathrm{~V}$

\section{Características radioeléctricas}

Banda de frecuencias radio

ISM $868 \mathrm{MHz}+$ ISM $434 \mathrm{MHz}$

Rango de frecuencias de transmisión

$868.0-868.6 \mathrm{MHz}$

Rango de frecuencias de recepción $869.4-869.65 \mathrm{MHz}$

Potencia de emisión

Ganancia de antena $14 \mathrm{dBm}(25 \mathrm{~mW})$

Tecnologías LPWAN soportadas

Sigfox

\begin{tabular}{ll}
\hline Certificación radio & Clase 0u \\
\hline Tasa de bits en transmisión & $100 \mathrm{bps}$ \\
\hline Modulación en transmisión & DBPSK \\
\hline Tasa de bits en recepción & $600 \mathrm{bps}$ \\
\hline Modulación en recepción & GFSK \\
\hline Sensibilidad de recepción & $-126 \mathrm{dBm}$ \\
\hline Consumo en transmisión & $52 \mathrm{~mA}$ \\
\hline Consumo en recepción & $13 \mathrm{~mA}$ \\
\hline Duración de transmisión con ack & $45 \mathrm{~s}$ \\
\hline LoRa & \\
\hline Certificación radio & LoRaWAN 1.0 Clase A \\
\hline Potencia de emisión & $14 \mathrm{dBm}(25 \mathrm{~mW})$ \\
\hline Tasa de bits emisión y recepción & $250-5470 \mathrm{bps}$ \\
\hline Sensibilidad de recepción & $-136 \mathrm{dBm}$ \\
\hline Consumo en transmisión & $38.9 \mathrm{~mA}$ \\
\hline Consumo en recepción & $14.2 \mathrm{~mA}$ \\
\hline
\end{tabular}

Tabla A1.11. Listado de componentes. 


\section{A1.6. Replicador de pulsos}

\section{A1.6.1. Listado de componentes}

\begin{tabular}{cllccccc}
\hline Ud & Descripción & Fabricante & Referencia & Distribuidor & Referencia & $€$ Ud & $\boldsymbol{\epsilon}$ \\
\hline 1 & Caja DIN & Phoenix Cont & 2202529 & Amidata & $909-3290$ & 8.71 & 8.71 \\
\hline 1 & PCB 100x50mm & C.I.F. & 8903575 & Amidata & $890-3575$ & 2.94 & 2.94 \\
\hline 8 & Relé est sólido & Panasonic & AQY212GH & Amidata & $811-3261$ & 5.73 & 45.84 \\
\hline 8 & Resistencia 220 & Vishay & MBB02070C22 & Amidata & $506-4863$ & 0.098 & 0.784 \\
& & & 00FCT00 & & & & \\
\hline 1 & Resistencia & Vishay & MBB02070C10 & Amidata & $477-7928$ & 0.082 & 0.082 \\
& $1 \mathrm{k} \Omega$ & 01FCT00 & & & & \\
\hline 1 & Resistencia & Vishay & MBB02070C10 & Amidata & $477-8246$ & 0.111 & 0.111 \\
& $10 \mathrm{k} \Omega$ & & 02FCT00 & & & & \\
\hline 1 & BC547 & ON Semi & BC547CTA & Amidata & $761-9822$ & 0.048 & 0.048 \\
\hline 1 & Batería CR2032 & Panasonic & CR-2032NCN & Amidata & $745-0875$ & 1.04 & 1.04 \\
\hline & & & & & & & 59.55
\end{tabular}

Tabla A1.12. Listado de componentes.

La versión SMD del relé de estado sólido tiene como referencia: Panasonic AQY212GHA

\section{A1.6.2. Etiqueta}

Las dimensiones de la etiqueta para la carcasa acoplable a carril DIN son: $4.1 \mathrm{~mm}$ (ancho) $\times 4.8$ $\mathrm{mm}$ (alto) y puede verse representada en la Figura A1.17.

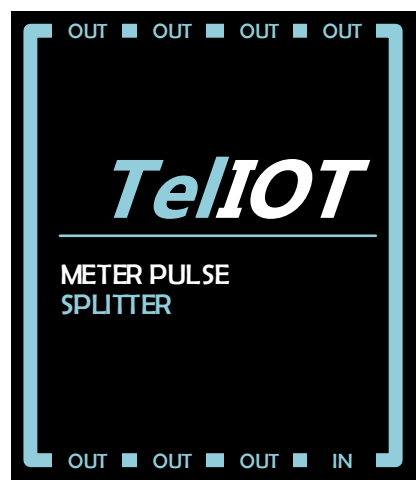

Figura A1.17. Etiqueta de panel frontal.

\section{A1.6.3. Especificaciones técnicas}

\begin{tabular}{l|l}
\hline Dimensiones físicas & $75.4 \times 45 \times 53.5 \mathrm{~mm}$ \\
\hline Alimentación & Pila interna CR2032 \\
\hline Tensión de alimentación & $3 \mathrm{~V}$ \\
\hline Consumo & $43 \mathrm{~mA}$ durante el impulso \\
\hline Tipo de entrada & Cierre de circuito a +3V \\
\hline Intensidad a la entrada & $0.5 \mathrm{~mA}$ \\
\hline Tensión a la entrada & $3 \mathrm{~V}$ \\
\hline Tipo de salida & Contacto libre de potencial
\end{tabular}


Intensidad máxima continua a la salida

$1.1 \mathrm{~A}$

Intensidad máxima de pico a la salida

Tensión de carga de pico en AC

Temperatura de funcionamiento

$3.0 \mathrm{~A}$

Temperatura de almacenamiento

$60 \mathrm{~V}$

$-40^{\circ} \mathrm{C} \mathrm{a}+85^{\circ} \mathrm{C}$

$-40^{\circ} \mathrm{C} \mathrm{a}+100^{\circ} \mathrm{C}$

Tabla A1.13. Listado de componentes.

\section{A1.7. Emulador de contador}

\section{A1.7.1. Listado de componentes}

\begin{tabular}{|c|c|c|c|c|c|c|c|}
\hline Ud & Descripción & Fabricante & $\begin{array}{c}\text { Referencia } \\
\text { fabricante }\end{array}$ & Distribuidor & $\begin{array}{l}\text { Referencia } \\
\text { distribuidor }\end{array}$ & $\begin{array}{l}\text { Precio } \\
\text { Ud }\end{array}$ & $\begin{array}{c}\text { Precio } \\
\text { Total } \\
\end{array}$ \\
\hline 1 & Arduino Uno & Arduino & A000066 & Amidata & $715-4081$ & 21,25 & 21,25 \\
\hline 1 & $\begin{array}{l}\text { PROTO SHIELD } \\
\text { REV3 (UNO SIZE) }\end{array}$ & Arduino & TSX00083 & Arduino & TSX00083 & 6,9 & 6,90 \\
\hline 1 & $\begin{array}{c}2.8 " 240 \times 320 \text { TFT } \\
\text { Touch }\end{array}$ & Adafruit & ADF-1651 & Electan & ADF-1651 & 42,03 & 42,03 \\
\hline 1 & $\begin{array}{c}\text { Resistencia } 470 \\
\text { ohmios } 1 / 4 \mathrm{~W}\end{array}$ & Vishay & $\begin{array}{c}\text { MRS25000 } \\
\text { C4703FCT00 }\end{array}$ & Amidata & $683-3730$ & 0,129 & 0,13 \\
\hline 1 & Relé estado sólido & Panasonic & $\mathrm{AQY} 212 \mathrm{GH}$ & Amidata & $811-3261$ & 5,73 & 5,73 \\
\hline 2 & $\begin{array}{l}\text { Regleta de } \\
\text { conexiones }\end{array}$ & $\begin{array}{l}\text { Phoenix } \\
\text { Contact }\end{array}$ & 1725656 & Amidata & $220-4260$ & 0,56 & 1,12 \\
\hline 1 & $\begin{array}{c}\text { Abrazaderas para } \\
\text { Carril DIN }\end{array}$ & Bopla & 22035000 & Amidata & $186-6788$ & 2,94 & 2,94 \\
\hline
\end{tabular}

Tabla A1.14. Listado de componentes.

\section{A1.7.2. Especificaciones técnicas}

\begin{tabular}{l|l}
\hline Dimensiones físicas & $75.4 \times 45 \times 53.5 \mathrm{~mm}$ \\
\hline Tensión de alimentación & $5 \mathrm{~V} \mathrm{DC}$ \\
\hline Consumo típico & $170 \mathrm{~mA}$ \\
\hline Tipo de salida & Contacto libre de potencial \\
\hline Intensidad máxima continua a la salida & $1.1 \mathrm{~A}$ \\
\hline Intensidad máxima de pico a la salida & $3.0 \mathrm{~A}$ \\
\hline Tensión de carga de pico en $\mathrm{AC}$ & $60 \mathrm{~V}$ \\
\hline Temperatura de funcionamiento & $-40^{\circ} \mathrm{C} \mathrm{a}+85^{\circ} \mathrm{C}$ \\
\hline Temperatura de almacenamiento & $-40^{\circ} \mathrm{C} \mathrm{a}+100^{\circ} \mathrm{C}$ \\
\hline
\end{tabular}

Tabla A1.15. Especificaciones técnicas. 
ANEXO 2

INFORMACIÓN ADICIONAL DEL FIRMWARE 



\section{A2.1. Dispositivo de contador con Waspmote}

En este apartado se incluye información complementaria referente al software embebido en el dispositivo de contador que incluye la placa base Waspmote. Comprende el listado de funciones utilizadas y las instrucciones necesarias para la programación del firmware.

\section{A2.1.1. Funciones utilizadas}

El listado de funciones a las que se hace llamada en el software embebido desarrollado, se encuentran clasificadas por tipo en la Tabla A2.1.

\section{Principal}

\begin{tabular}{|l|l|l|}
\hline Prototipo & void setup(void) \\
\hline Descripción & $\begin{array}{l}\text { Se ejectuta la primera vez que se enciende el dispositivo } \\
\text { Configura el hardware e inicializa variables }\end{array}$ \\
\hline Devuelve & void & \\
\hline Argumento 1 & void & \\
\hline
\end{tabular}

\begin{tabular}{|l|l|l|}
\hline Prototipo & \multicolumn{2}{|l|}{ void loop(void) } \\
\hline Descripción & Bucle principal de ejecución \\
\hline Devuelve & void & \\
\hline Argumento 1 & void & \\
\hline
\end{tabular}

\begin{tabular}{|l|l|l|}
\hline Prototipo & \multicolumn{2}{|l|}{ void LedTx(bool bSt) } \\
\hline Descripción & Cambia de estado el indicador led \\
\hline Devuelve & void & \\
\hline Argumento 1 & bool & Nuevo estado de la salida \\
\hline
\end{tabular}

\section{Comunicaciones}

\begin{tabular}{|l|l|l|}
\hline Prototipo & void SendData(void) \\
\hline Descripción & Envía una trama de datos \\
\hline Devuelve & void & \\
\hline Argumento 1 & void & \\
\hline Prototipo & void ProcessRx(void) \\
\hline Descripción & Procesa los datos recibidos en una trama de bajada \\
\hline Devuelve & void & \\
\hline Argumento 1 & void & \\
\hline
\end{tabular}

Unidades de comunicación Sigfox / LoRaWAN / LoRa2Gate

\begin{tabular}{|l|l|l|}
\hline Prototipo & \multicolumn{2}{l|}{ uint8_t SendRadio(uint8_t SendData[], uint8_t } \\
& SendData_Len, bool bACK) \\
\hline Descripción & Envía una trama de datos via radio \\
\hline Devuelve & void & \\
\hline Argumento 1 & uint8_t[] & Datos a enviar \\
\hline Argumento 2 & uint8_t & Cantidad de datos a enviar \\
\hline Argumento 3 & bool & Solicitud de trama de bajada \\
\hline
\end{tabular}




\begin{tabular}{|c|c|}
\hline Prototipo & $\begin{array}{l}\text { bool ExtractRxData(char }{ }^{*} \text { rxData, int iRxDataLen, int } \\
\text { iDataout []) }\end{array}$ \\
\hline Descripción & Extrae la información útil de la trama recibida \\
\hline Devuelve & \begin{tabular}{|l|l|} 
bool & True: $0 k$ \\
& False: Error
\end{tabular} \\
\hline Argumento 1 & Cadena de texto recibida \\
\hline Argumento 2 & Longitud de la cadena recibida \\
\hline Argumento & Datos extraídos \\
\hline Prototipo & uint8_t SetupLoRaModule() \\
\hline Descripción & Inicializa el modulo LoRaWAN para su uso en modo LoRa \\
\hline Devuelve & \begin{tabular}{l|l} 
uint8_t & $\begin{array}{l}\text { : Ok } \\
>\odot: \text { Código de error }\end{array}$ \\
\end{tabular} \\
\hline
\end{tabular}

\section{Operación}

\begin{tabular}{|l|l|l|}
\hline Prototipo & void SetTxPeriod(int iUnits, int iPeriod) \\
\hline Descripción & Establece un nuevo intervalo de envío de datos \\
\hline Devuelve & void & \multicolumn{1}{|l|}{} \\
\hline Argumento 1 & int & $\begin{array}{l}\text { 1:Minutos } \\
\text { 2:Horas } \\
\text { 3:Días }\end{array}$ \\
\hline Argumento 2 & int & Intervalo de tiempo según unidades \\
\hline Prototipo & bool RequestAck() \\
\hline Descripción & $\begin{array}{l}\text { Comprueba si es posible solicitar trama de bajada o se han } \\
\text { superado los límites permitidos }\end{array}$ \\
\hline Devuelve & bool & $\begin{array}{l}\text { True: Ack posible } \\
\text { False: Ack consumidos }\end{array}$ \\
\hline Argumento 1 & void & \\
\hline
\end{tabular}

\begin{tabular}{|l|l|l|}
\hline Prototipo & bool RequestAck() \\
\hline Descripción & $\begin{array}{l}\text { Comprueba si es posible solicitar trama de bajada o se han } \\
\text { superado los límites permitidos }\end{array}$ \\
\hline Devuelve & bool & $\begin{array}{l}\text { True: Ack posible } \\
\text { False: Ack consumidos }\end{array}$ \\
\hline Argumento 1 & void & \\
\hline
\end{tabular}

\begin{tabular}{|l|l|l|l|}
\hline Prototipo & \multicolumn{4}{|l|}{ int GetLastACKDay( ) } \\
\hline Descripción & Obtiene el día que se solicitó la última trama de bajada \\
\hline Devuelve & int & Número del día que se solicitó trama de bajada \\
\hline Argumento 1 & void & \\
\hline
\end{tabular}

\begin{tabular}{|l|l|l|}
\hline Prototipo & \multicolumn{4}{|l|}{ void SetLastACKDay(int iDay) } \\
\hline Descripción & Guarda el día que se ha solicitado trama de bajada \\
\hline Devuelve & void & \\
\hline Argumento 1 & int & $\begin{array}{l}\text { Número del día que se ha solicitado la última } \\
\text { trama de bajada }\end{array}$ \\
\hline
\end{tabular}

\section{RTC}

\begin{tabular}{|l|l|l|}
\hline Prototipo & \multicolumn{2}{|l|}{ void SetNewTime(uint32_t lEpoch) } \\
\hline Descripción & Configura una nueva hora en el RTC \\
\hline Devuelve & void & \\
\hline Argumento 1 & uint32_t & Nuevo tiempo en formato epoch \\
\hline
\end{tabular}




\section{Salida}

\begin{tabular}{|l|l|l|}
\hline Prototipo & \multicolumn{2}{|l|}{ void Eletrovalve(bool bout) } \\
\hline Descripción & Fija el estado de la salida de control \\
\hline Devuelve & void & \\
\hline Argumento 1 & bool & Nuevo estado de la salida \\
\hline
\end{tabular}

\section{Utilidades}

\begin{tabular}{|c|c|}
\hline Prototipo & void Save_Counter_EE() \\
\hline Descripción & $\begin{array}{l}\text { Guarda en la e2prom el valor del contador } \\
\text { Variable global utilizada: luPulsecounter }\end{array}$ \\
\hline Devuelve & \begin{tabular}{l|l} 
Void & \\
\end{tabular} \\
\hline Prototipo & void Read_Counter_EE( ) \\
\hline Descripción & $\begin{array}{l}\text { Lee de la e2prom el valor del contador } \\
\text { Variable global utilizada: lupulsecounter }\end{array}$ \\
\hline Devuelve & \begin{tabular}{l|l} 
void & \\
\end{tabular} \\
\hline Prototipo & void LedTx(bool bSt) \\
\hline Descripción & Cambia de estado el indicador led \\
\hline Devuelve & \begin{tabular}{l|l} 
void &
\end{tabular} \\
\hline Argumento 1 & \begin{tabular}{l|l} 
bool & Nuevo estado de la salida \\
\end{tabular} \\
\hline Prototipo & void LedRx(bool bSt) \\
\hline Descripción & Cambia de estado el indicador led \\
\hline Devuelve & \begin{tabular}{l|l} 
Void & \\
\end{tabular} \\
\hline Argumento 1 & Nuevo estado de la salida \\
\hline
\end{tabular}

\section{Depuración}

\begin{tabular}{|c|c|}
\hline Prototipo & void DebugInit () \\
\hline Descripción & Inicializa el puerto usb para la depuración \\
\hline Devuelve & \begin{tabular}{l|l} 
void & \\
\end{tabular} \\
\hline Prototipo & void DebugEnd() \\
\hline Descripción & Cierra el puerto usb para la depuración \\
\hline Devuelve & \begin{tabular}{l|l} 
void & . \\
\end{tabular} \\
\hline
\end{tabular}

\begin{tabular}{|l|l|l|}
\hline Prototipo & \multicolumn{1}{|l|}{ void DebugString(const _FlashstringHelper* str) } \\
\hline Descripción & Envía una cadena de texto al interface de depuración \\
\hline Devuelve & void & \\
\hline Argumento 1 & const _FlashStringHelper ${ }^{*}$ & Texto \\
\hline Prototipo & void Debugstring(char * ${ }^{*}$ str) \\
\hline Descripción & Envía una cadena de texto al interface de depuración \\
\hline Devuelve & void & \\
\hline Argumento 1 & char ${ }^{*}$ & Texto \\
\hline
\end{tabular}

\section{LoRa}

\begin{tabular}{|l|l|}
\hline Prototipo & uint8_t LoRa_SetupModule( ) \\
\hline Descripción & Inicializa el modulo LoRaWAN para su uso en modo LoRa \\
\hline Devuelve & uint8_t $\begin{array}{l}\text { O: Ok } \\
>\odot: \text { Código de error }\end{array}$ \\
\hline
\end{tabular}




\begin{tabular}{|c|c|}
\hline Prototipo & uint8_t LoRaWAN.OFF(uint8_t socket) \\
\hline Descripción & Finaliza el uso del módulo radio \\
\hline Devuelve & \begin{tabular}{l|l} 
uint8_t & $\odot:$ Ok \\
& $>\odot:$ Código de error
\end{tabular} \\
\hline Argumento 1 & $\begin{array}{l}\text { Puerto al que está conectado el módulo: } \\
\text { SOCKET@ } \\
\text { SOCKET1 }\end{array}$ \\
\hline
\end{tabular}

\begin{tabular}{|l|l|l|}
\hline Prototipo & \multicolumn{2}{|l|}{ uint8_t LoRaWAN.resetMacConfig(char ${ }^{*}$ ) } \\
\hline Descripción & \multicolumn{2}{|l|}{ Establece la banda de frecuencias } \\
\hline Devuelve & uint8_t & $\begin{array}{l}\text { 0: 0k } \\
>0: \text { Código de error }\end{array}$ \\
\hline Argumento 1 & char * & $\begin{array}{l}\text { "433" } \\
\text { "868" }\end{array}$ \\
\hline
\end{tabular}

\begin{tabular}{|l|l|l|}
\hline Prototipo & \multicolumn{2}{|l|}{ uint8_t LoRaWAN.macPause( ) } \\
\hline Descripción & Usa LoRa en lugar de LoRaWAN \\
\hline Devuelve & uint8_t \begin{tabular}{l}
$\begin{array}{l}0: \text { Ok } \\
>0: \text { Código de error }\end{array}$ \\
\hline Argumento 1
\end{tabular} int & Tiempo máximo a esperar en ms \\
\hline
\end{tabular}

\begin{tabular}{|l|l|l|}
\hline Prototipo & \multicolumn{2}{|l|}{ uint8_t LoRaWAN.setRadioFreq(long) } \\
\hline Descripción & \multicolumn{2}{|l|}{ Establece la frecuencia de operación } \\
\hline Devuelve & uint8_t & $\begin{array}{l}\text { }: \text { Ok } \\
>0: \text { Código de error }\end{array}$ \\
\hline Argumento 1 & long & Frecuencia en Hz \\
\hline
\end{tabular}

\begin{tabular}{|l|l|l|l|}
\hline Prototipo & \multicolumn{4}{|l|}{ uint8_t LoRaWAN.setRadioPower(int) } \\
\hline Descripción & \multicolumn{2}{|l|}{ Establece la potencia de transmisión } \\
\hline Devuelve & uint8_t & $\begin{array}{l}\odot: \text { Ok } \\
>0: \text { Código de error }\end{array}$ \\
\hline Argumento 1 & int & Nivel de potencia: -3 a 15 \\
\hline
\end{tabular}

\begin{tabular}{|l|l|l|}
\hline Prototipo & uint8_t LoRaWAN.setRadioSF $\left(\right.$ char ${ }^{*}$ ) \\
\hline Descripción & Establece el factor de esparcimiento \\
\hline Devuelve & uint8_t & $\begin{array}{l}\text { ○: Ok } \\
>0 \text { : Código de error }\end{array}$ \\
\hline Argumento 1 & char * & $\begin{array}{l}\text { Spreading Factor } \\
\text { "sf7" } \\
\text { "sf8" }\end{array}$ \\
& & $\begin{array}{l}\text { "sf9" } \\
\text { "sf10" } \\
\text { "sf11" } \\
\text { "sf12" }\end{array}$ \\
\hline
\end{tabular}

\begin{tabular}{|l|l|l|}
\hline Prototipo & uint8_t LoRaWAN. SetRadioCR(char ${ }^{*}$ ) \\
\hline Descripción & Establece el ratio de codificación de la comunicación \\
\hline Devuelve & uint8_t & $\begin{array}{l}0: \text { : Ok } \\
>0: \text { Código de error }\end{array}$ \\
\hline Argumento 1 & char * & $\begin{array}{l}\text { Coding rate } \\
\text { "4/5" } \\
\end{array}$ \\
& & "4/6" \\
& & $4 / 7$ " \\
& & $4 / 8$ " \\
\hline
\end{tabular}




\begin{tabular}{|c|c|c|}
\hline Prototipo & \multicolumn{2}{|c|}{ uint8_t LoRaWAN.setRadioBandwidth(int) } \\
\hline Descripción & \multicolumn{2}{|c|}{ Establece el ancho de banda } \\
\hline Devuelve & uint8_t & $\begin{array}{l}\text { ๑: Ok } \\
>\odot: \text { Código de error }\end{array}$ \\
\hline Argumento 1 & int & $\begin{array}{l}\text { Ancho de banda en } \mathrm{KHz} \\
125 \\
250 \\
500\end{array}$ \\
\hline
\end{tabular}

\begin{tabular}{|l|l|l|}
\hline Prototipo & uint8_t LoRaWAN . setRadioCRC(char ${ }^{*}$ ) \\
\hline Descripción & $\begin{array}{l}\text { Activa el envío de un chequeo de redundancia cíclica CRC } \\
\text { de los datos }\end{array}$ \\
\hline Devuelve & uint8_t $\begin{array}{l}\text { ०: Ok } \\
>\odot: \text { Código de error }\end{array}$ \\
\hline Argumento 1 & char * & $\begin{array}{l}\text { "on": Activado } \\
\text { "off": Desactivado }\end{array}$ \\
\hline
\end{tabular}

\section{Librería de comunicaciones Sigfox}

\begin{tabular}{|l|l|l|}
\hline Prototipo & uint8_t Sigfox.on(uint8_t socket) \\
\hline Descripción & Inicializa el módulo radio para su uso \\
\hline Devuelve & uint8_t & $\begin{array}{l}\text { 0: Ok } \\
>0: \text { Código de error }\end{array}$ \\
\hline Argumento 1 & uint8_t & $\begin{array}{l}\text { Puerto al que está conectado el módulo } \\
\text { SOCKET० } \\
\text { SOCKET1 }\end{array}$ \\
\hline
\end{tabular}

\begin{tabular}{|l|l|l|}
\hline Prototipo & uint8_t Sigfox.send(uint8_t data, int len) \\
\hline Descripción & Envía una trama de datos sin esperar confirmación \\
\hline Devuelve & uint8_t & $\begin{array}{l}\text { ○: Ok } \\
>\odot: \text { Código de error }\end{array}$ \\
\hline Argumento 1 & uint8_t & Datos a enviar \\
\hline Argumento 2 & int & Cantidad de datos a enviar \\
\hline
\end{tabular}

\begin{tabular}{|l|l|l|}
\hline Prototipo & \multicolumn{1}{|l|}{ uint8_t Sigfox.sendACK(uint8_t data, int len) } \\
\hline Descripción & Envía una trama y espera confirmación y datos de bajada \\
\hline Devuelve & uint8_t & $\begin{array}{l}\text { 0: Ok } \\
>0: \text { Código de error }\end{array}$ \\
\hline Argumento 1 & uint8_t & Datos a enviar \\
\hline Argumento 2 & int & Cantidad de datos a enviar \\
\hline
\end{tabular}

\begin{tabular}{|l|l|l|}
\hline Prototipo & \multicolumn{2}{|l|}{ uint8_t Sigfox.0FF(uint8_t socket) } \\
\hline Descripción & Finaliza el uso del módulo radio \\
\hline Devuelve & uint8_t & $\begin{array}{l}\text { 0: Ok } \\
>0: \text { Código de error }\end{array}$ \\
\hline Argumento 1 & uint8_t & $\begin{array}{l}\text { Puerto al que está conectado el módulo } \\
\text { SOCKET॰ } \\
\text { SOCKET1 }\end{array}$ \\
\hline
\end{tabular}

Tabla A2.1. Listado de funciones del software embebido en Waspmote. 


\section{A2.1.2. Programación del firmware}

El firmware se encuentra estructurado en los siguientes archivos:

- Main.pde : Funciones principales

- Debug.pde: Funciones para depuración

- LoRa.pde : Funciones para el uso del módulo radio LoRa

- Sigfox.pde : Funciones para el uso del módulo radio Sigfox

En el archivo Main.pde están disponibles las siguientes opciones que si se considera oportuno deberán modificarse antes de la compilación y carga del código en el dispositivo.

Activación del modo de depuración. Envía información adicional por el interface serie virtual del puerto USB sobre la operación en curso y su resultado.

\#define DEBUG

1

Intervalo por defecto en minutos del envío de datos a través de radio.

\#define SENDDATA_DEF_MINS 10

Realizar un primer envío de datos y recogida de información al encenderse.

\#define FIRST_TX

1

Forzar a que todas las transmisiones soliciten una trama de bajada de configuración.

\#define FORCE_ACK

$\odot$

Identificador de dispositivo para el caso de conexión a través de gateway.

\#define SUBDEVICE

3

Entre los modos de operación Sigfox/LoRaWan/LoRa, sólo uno de ellos puede estar activo a la vez.

$\begin{array}{lll}\text { \#define } & \text { MODE_SIGFOX } & \odot \\ \text { \#define } & \text { MODE_LORAWAN } & \odot \\ \text { \#define MODE_LORA2GATE } & 1\end{array}$

La carga del firmware en el dispositivo se hace desde el entorno de edición y programación Waspmote Pro IDE. El procedimiento a seguir es el descrito a continuación:

- Menú Archivo $\rightarrow$ Abrir $\rightarrow$ Main.pde 


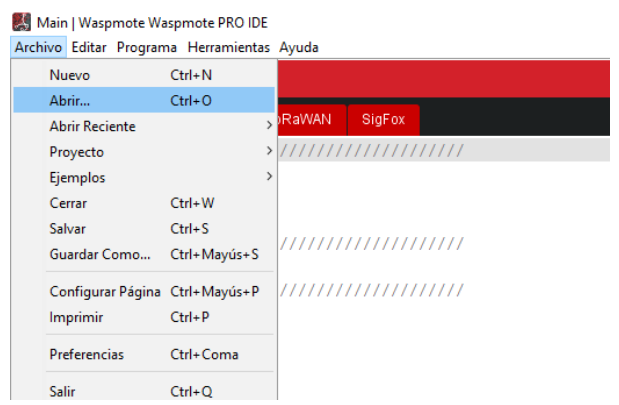

Figura A2.1. Selección de archivo.

- Menú Herramientas $\rightarrow$ Placa $\rightarrow$ Waspmote API

\begin{tabular}{l} 
Main / Waspmote Waspmote PRO IDE \\
Archivo Editar Programa Herramientas Ayuda \\
\hline
\end{tabular}

Figura A2.2. Selección del tipo de placa.

- Menú Herramientas $\rightarrow$ Puerto $\rightarrow$ COMxx (variable según el hardware del PC)

\begin{tabular}{|c|c|c|c|}
\hline Archivo Editar Programa & Herramientas Ayuda & & \\
\hline 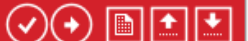 & Auto Formato & $\mathrm{Ctrl}+\mathrm{T}$ & \\
\hline \begin{tabular}{l|l|l} 
Main & Debug & Lof
\end{tabular} & $\begin{array}{l}\text { Archivo de programa. } \\
\text { Reparar codificación \& Recargar. }\end{array}$ & & \\
\hline $1 / / / / / / / / / / / / / 1$ & Monitor Serie & Ctrl+Mayús+M & \\
\hline // TELIOT PROJECT & Serial Plotter & Ctrl+Mayús+L & \\
\hline $\begin{array}{l}\text { // WASPMOTE CODE } \\
\text { // METER VIA GATEV }\end{array}$ & Placa: "Waspmote API v028" & $>$ & \\
\hline$/ / / / / / / / / / / / / / 1$ & Puerto & ; & Puertos Serie \\
\hline // MAIN & Obtén información de la placa & & COM6 \\
\hline
\end{tabular}

Figura A2.3. Selección del puerto serie.

- Menú Programa $\rightarrow$ Subir

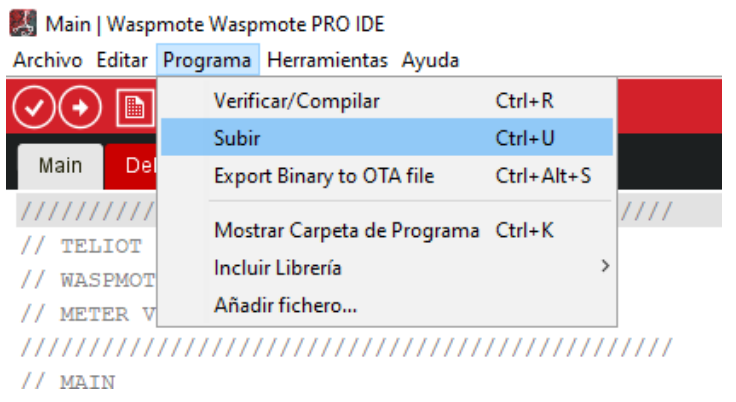

Figura A2.4. Programación del firmware. 


\section{A2.2. Dispositivo de contador con Pycom}

En este apartado se incluye información complementaria referente al software embebido en el dispositivo de contador que incluye el controlador Pycom. Comprende el listado de funciones utilizadas y las instrucciones de programación del firmware.

\section{A2.2.1. Funciones utilizadas}

El listado de funciones a las que se hace llamada en el software embebido desarrollado, se encuentran clasificadas por tipo en la Tabla A2.2.

\section{Comunicaciones}

\begin{tabular}{|l|l|l|}
\hline Prototipo & \multicolumn{2}{|l|}{ SendData(rx_request) } \\
\hline Descripción & Envía una trama de datos con o sin solicitud de bajada \\
\hline Devuelve & bool & $\begin{array}{l}\text { True: Trama de bajada } \\
\text { False: Sin trama de bajada }\end{array}$ \\
\hline Argumento 1 & bool & Solicitud de trama de bajada \\
\hline Prototipo & ProcessRx(char ${ }^{*}$ ) \\
\hline Descripción & Procesa los datos recibidos en una trama de bajada \\
\hline Devuelve & bool & $\begin{array}{l}\text { True: Trama de bajada ok } \\
\text { False: Error en la trama }\end{array}$ \\
\hline Argumento 1 & char & Datos recibidos \\
\hline
\end{tabular}

\section{Gestión del intervalo entre transmisiones}

\begin{tabular}{|l|l|l|}
\hline Prototipo & \multicolumn{3}{|l|}{ GetTxInterval() } \\
\hline Descripción & Devuelve el periodo configurado para envío de datos \\
\hline Devuelve & int & Número de minutos para envío de datos \\
\hline Argumento 1 & void & \\
\hline
\end{tabular}

\begin{tabular}{|l|l|l|}
\hline Prototipo & \multicolumn{3}{|l|}{ SetTxInterval(New_TxInterval_Mins) } \\
\hline Descripción & Configura el intervalo para envío de datos \\
\hline Devuelve & void & \\
\hline Argumento 1 & int & Número de minutos para envío de datos \\
\hline
\end{tabular}

\begin{tabular}{|l|l|l|}
\hline Prototipo & \multicolumn{3}{|l|}{ GetMins2Tx( ) } \\
\hline Descripción & Devuelve los minutos transcurridos para envío de datos \\
\hline Devuelve & int & Número de minutos para envío de datos \\
\hline Argumento 1 & void & \\
\hline
\end{tabular}

\begin{tabular}{|l|l|l|}
\hline Prototipo & \multicolumn{3}{|l|}{ SetMins2Tx(New_Elapsed_Mins) } \\
\hline Descripción & Almacena los minutos transcurridos para envío de datos \\
\hline Devuelve & void & \\
\hline Argumento 1 & int & Número de minutos para envío de datos \\
\hline
\end{tabular}


Gestión del intervalo entre transmisiones con confirmación

\begin{tabular}{|c|c|}
\hline Prototipo & IsAckRequired() \\
\hline Descripción & Indica si ha de solicitarse trama de bajada \\
\hline Devuelve & \begin{tabular}{|l|l} 
bool & True: $\mathrm{TX}+\mathrm{ACK}$ \\
& False: $\mathrm{TX}$
\end{tabular} \\
\hline Argumento 1 & void \\
\hline Prototipo & GetMinsSinceAck ( ) \\
\hline Descripción & Configura el intervalo para envío de datos \\
\hline Devuelve & \begin{tabular}{l|l} 
void & \\
\end{tabular} \\
\hline Argumento 1 & \begin{tabular}{l|l} 
int & Número de minutos para envío de datos \\
\end{tabular} \\
\hline Prototipo & ResetMinsSinceAck ( ) \\
\hline Descripción & Pone a cero el contador de minutos desde el último ack \\
\hline Devuelve & \begin{tabular}{l|l} 
void & \multicolumn{2}{|}{} \\
\end{tabular} \\
\hline Argumento 1 & void \\
\hline Prototipo & IncreaseMinsSinceAck ( ) \\
\hline Descripción & Almacena los minutos transcurridos para envío de datos \\
\hline Devuelve & \begin{tabular}{l|l} 
void & \\
\end{tabular} \\
\hline Argumento 1 & Número de minutos para envío de datos \\
\hline
\end{tabular}

\section{Salida}

\begin{tabular}{|l|l|l|}
\hline Prototipo & \multicolumn{3}{|l|}{ void EValve(bool bout ) } \\
\hline Descripción & Fija el estado de la salida de control \\
\hline Devuelve & void & \\
\hline Argumento 1 & bool & Nuevo estado de la salida \\
\hline
\end{tabular}

\section{Contador}

\begin{tabular}{|l|l|l|}
\hline Prototipo & GetMeterCounter( ) \\
\hline Descripción & Obtiene el valor actual del contador almacenado \\
\hline Devuelve & void & \\
\hline Argumento 1 & bool & Nuevo estado de la salida \\
\hline Prototipo & SetMeterCounter(New_MeterValue) \\
\hline Descripción & Obtiene el valor actual del contador almacenado \\
\hline Devuelve & void & \\
\hline Argumento 1 & Int32 & Nuevo valor del contador \\
\hline
\end{tabular}

\section{Modo de bajo consumo}

\begin{tabular}{|l|l|}
\hline Prototipo & Go2sleep ( ) \\
\hline Descripción & $\begin{array}{l}\text { Pasa al modo de bajo consumo activando las opciones que } \\
\text { pueden volver a despertar al módulo }\end{array}$ \\
\hline Devuelve & void \\
\hline
\end{tabular}

\section{Depuración}

\begin{tabular}{|l|l|l|}
\hline Prototipo & \multicolumn{2}{|l|}{ void Debugusb(char *str) } \\
\hline Descripción & Envía una cadena de texto al interface de depuración \\
\hline Devuelve & void & \multicolumn{2}{|l|}{} \\
\hline Argumento 1 & char ${ }^{\star}$ & Texto \\
\hline
\end{tabular}




\section{Sigfox}

\begin{tabular}{|l|l|l|}
\hline Prototipo & \multicolumn{3}{|l|}{ Sigfox_Send(char *data, bool rx_request) } \\
\hline Descripción & Envía una trama de datos usando la red Sigfox \\
\hline Devuelve & int & Longitud de la trama de datos recibida \\
\hline Argumento 1 & char * & Datos a enviar \\
\hline Argumento 2 & bool & Solicitud de ack \\
\hline
\end{tabular}

\section{LoRaWAN}

\begin{tabular}{|l|l|l|}
\hline Prototipo & \multicolumn{3}{|l|}{ LoRaWANSend(char * ${ }^{*}$ data, bool rx_request) } \\
\hline Descripción & Envía una trama de datos usando una red LoRaWAN \\
\hline Devuelve & int & Longitud de la trama de datos recibida \\
\hline Argumento 1 & char * & Datos a enviar \\
\hline Argumento 2 & bool & Solicitud de ack \\
\hline
\end{tabular}

Tabla A2.2. Listado de funciones del software embebido en Pycom.

\section{A2.2.2. Programación del firmware}

El software embebido para esta aplicación se encuentra disponible en el archivo 'teliot.py' y se ha de cargar en el módulo para su ejecución siguiendo el procedimiento a continuación descrito. Si además se desea que el código se ejecute en cada inicio, se han de realizar unos pasos adicionales.

La programación del firmware en el dispositivo se lleva a cabo con el editor Atom de código abierto, apoyado por un complemento o plug-in para comunicar con los módulos Pycom llamado Pymakr. Pueden obtenerse a través de los siguientes enlaces:

- Atom: https://atom.io

- Pymakr Atom Package: https://atom.io/packages/pymakr

El complemento Pymakr puede añadirse desde dentro del mismo entorno. Cabe la posibilidad de problemas de compatibilidad entre versiones de ambos. Una combinación verificada es Atom $1.20+$ Pymakr 1.1 .13

Para instalar automáticamente la última versión del plugin, el procedimiento es el siguiente:

- Instalar Atom IDE. Queda en IUsers\\#lappdatallocallatom

- Añadir Pymakr desde Atom en el menú Settings $\rightarrow$ Packages $\rightarrow$ Find: pymakr

Para una instalación manual del plugin (debido a una posible incompatibilidad de versiones), el procedimiento es:

- Borrar C:IUsersi\#l.atom

- Borrar C:IUsersl\#lappdatallocallatom

- Instalar Atom 1.20

- Descomprimir pymakr-atom-1.1.3.zip en C:IUsers|\#l.atom|packages|

- Desde la línea de comandos compilar e instalar el plugin con estos comandos:

C: \Users \\#\. atom\packages \pymakr -atom-1.1.3> 


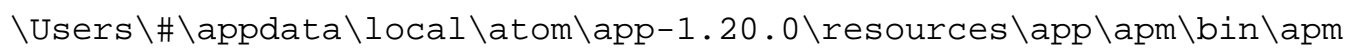
apm link

apm install

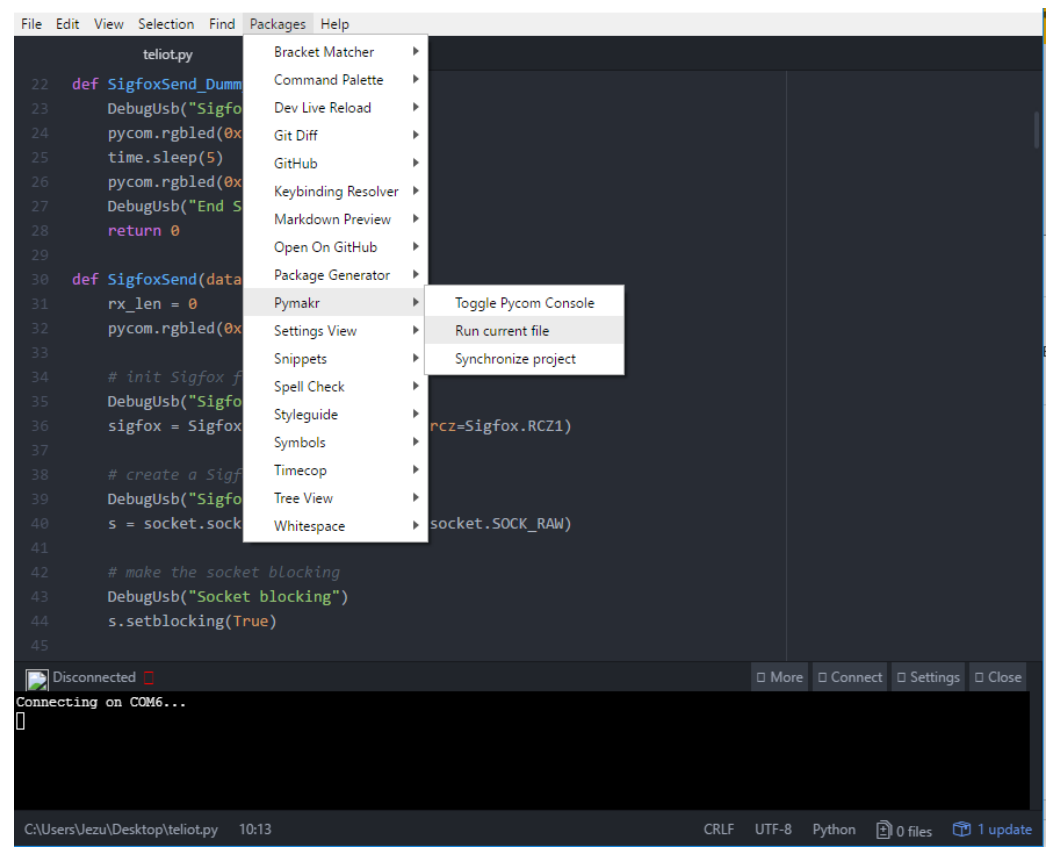

Figura A2.5. Editor Atom y consola Pymakr.

Para la puesta en marcha se han de seguir los siguientes pasos:

Preparación de módulo:

- En caso de módulos SiPy/LoPy versión 1.0, pinchar el módulo sobre la Deepsleep Shield (marca de serigrafía hacia LED)

- Pinchar en la placa de expansión (LED hacia USB)

- Conectar cable de antena 868 al U.FL junto al LED

- Conectar cable USB en la placa de expansión

- Verificar led azul parpadeante

Para cargar el código en el módulo y verificar funcionamiento:

- Menú: File $\rightarrow$ Open $\rightarrow$ teliot.py

- Menú: Packages $\rightarrow$ Pymakr $\rightarrow$ Toggle Pycom Console

- Pycom Console: Botón Connect

- Menú: Packages $\rightarrow$ Pymakr $\rightarrow$ Run current File

Para preparar el módulo de manera que ejecute el código en cada inicio, es necesario acceder al sistema de archivos. El módulo puede crear un servicio FTP al que conectar a través de la red WiFi. El procedimiento es el siguiente:

- Iniciar Atom

- Iniciar consola Pycom (Menu Packages -> Pymakr -> Toggle Pycom Console)

- Conectar consola (Botón Connect) 
- Asegurar que los servicios IP del módulo se inician. Para ello ejecutar los comandos:

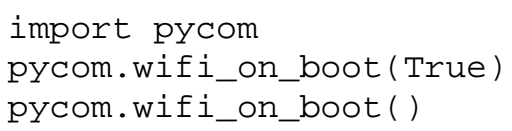

- Reiniciar con pulsador o desconectando alimentación (USB)

- Conectar el módulo a la red WiFi existente. Para ello abrir en Atom el código wifi_cnx.py y modificar las credenciales para adaptarlo a cada caso.

- Ejecutar wifi_cnx.py (Packages -> Pymakr -> Run current file)

- Acceder por FTP desde la misma red WiFi con un cliente como Filezilla. Los datos de conexión son los siguientes:

- Usuario: micro

- Password: Python

- Modo pasivo

- Sólo una conexión simultánea

- Transferir los archivos boot.py y teliot.py al directorio raíz

- Transferir al directorio 'lib' la librería deepsleep.py para poder utilizar la placa Deepsleep Shield

- Desconectar el cliente ftp y reiniciar el módulo.

Para verificar:

- Desconectar + Conectar USB

- Debe encenderse led amarillo durante $1 \mathrm{sg}$

- Debe iniciar una transmisión (led rojo)

- Verificar trama recibida en Sigfox backend

Instalación del módulo en el dispositivo final:

- Desconectar USB

- Extraer módulo de la placa de expansión

- Insertar módulo en placa Teliot (LED hacia lado opuesto a regletas)

Para llevar actualizaciones del firmare, el material necesario es el siguiente:

- Placa de expansión de Pycom

- Cable USB

- Entorno de desarrollo ATOM+Pymakr instalado

Seguir el siguiente procedimiento para actualizar el software embebido en el dispositivo:

1. Desconectar alimentación

2. Pinchar módulo en placa de expansión (LED hacia USB)

3. Puente entre $\mathrm{G} 28$ y $3 \mathrm{~V} 3$

4. Conectar alimentación

5. Al encenderse led naranja quitar puente

6. Atom: Establecer conexión consola

7. Atom: Abrir wifi_cnx.py

8. Atom: Cargar y ejecutar wifi_cnx.py (Packages -> Pymakr -> Run current file)

9. Filezilla: Conectar por FTP 
10. Filezilla: Transferir y sobreescribir teliot.py

11. Atom: Abrir teliot.py

12. Atom: Cargar y ejecutar teliot.py (Packages -> Pymakr -> Run current file)

\section{A2.3. Estación base LoRaWAN}

En este apartado se incluye información complementaria para la puesta en marcha de la estación base LoRaWAN de desarrollo propio.

\section{A2.3.1. Instalación del software y puesta en marcha}

La instalación y puesta en marcha del software embebido conlleva los siguientes pasos:

- Grabar la última versión de Raspbian en la tarjeta SD:

- https://www.raspberrypi.org/downloads/raspbian/

- Herramienta para grabación de la imagen en la SD: Win32Disklmager

- Iniciar el sistema

- Activar el bus SPI:

- sudo raspi-config

- Interfacing options $\rightarrow$ Enable SPI

- Actualizar Raspbian:

- sudo apt-get update

- sudo apt-get upgrade

- Instalar git:

- sudo apt-get install git

- sudo apt-get install git-core

- Descargar el código del gateway:

- git clone https://github.com/Lora-net/packet_forwarder

- git clone https://github.com/Lora-net/lora_gateway

- Compilar el gateway:

- cd lora_gateway

- sudo make all

- cd packet_forwarder

- sudo make all

- Configurar el ID del gateway:

- cd packet_forwarder/lora_pkt_fwd

- ./update_gid.sh local_conf.json

- Configurar los datos del servidor:

- vi global_conf.json 
- Modificar: gateway_ID, server address, serv_port_up, serv_port_down

Tras la conexión de la alimentación se hace necesario un reinicio del hardware del concentrador cambiando de estado lógico una de las señales GPIO. El procedimiento se lleva a cabo con la ayuda de un script. El pin de reset del concentrador (13) está conectado por la placa de adaptación al GPIO25 de Raspberry.

Contenido del script:

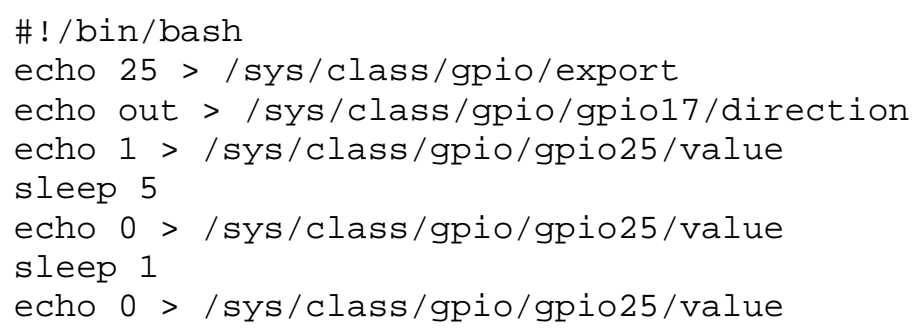

Se ha de crear el script y grabarlo en la siguiente localización:

packet_forwarder/lora_pkt_fwd/reset.sh

Para iniciar el software de gateway para LoRa se han de ejecutar los siguientes comandos:

.$/$ reset.sh

./lora_pkt_fwd

\section{A2.4. Entorno de validación en laboratorio}

En este apartado se incluye información complementaria referente a la consola con diferentes elementos loT, distribuidor de pulsos y emulador de contador, construida para pruebas de validación en laboratorio.

\section{A2.4.1. Formato de las tramas enviadas}

El dispositivo envía cuatro diferentes tipos de tramas, siendo las de tipo 2 las que contienen la información de consumo y son enviadas periódicamente, mientras que las restantes indican la configuración establecida y son únicamente enviadas ante una modificación de esta.

Las diferentes tramas siguen el siguiente formato.

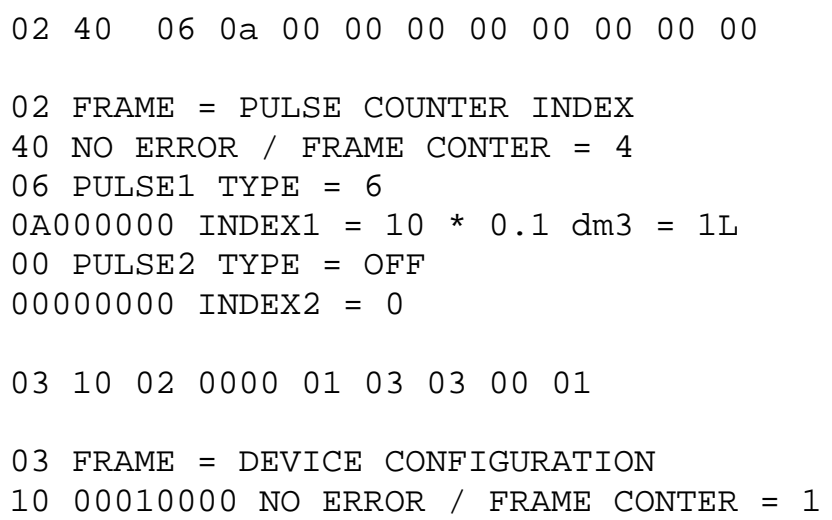


○2 DEVICE TYPE = PULSE

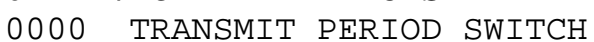

$01 \mathrm{CH} 1 \mathrm{ON} / \mathrm{CH} 2 \mathrm{OFF}$

○3 $\mathrm{CH} 1$ TYPE = PULSE AUTO

○3 $\mathrm{CH} 2$ TYPE = PULSE AUTO

$\odot \odot$ PULSE INPUT TYPE = AUTO

$\odot 1$ MEMO SWITCH $=1$

$0420 \quad 000 \odot \quad \odot а \odot \odot \odot \odot \odot \odot$

$\odot 4$ FRAME $=$ PULSE COUNTER 1 CONFIG

20 NO ERROR / FRAME CONTER $=2$

๑९९ WEIGHT $=$ SWITCH

๑а๑९९९९९ OFFSET $=10=0.1 \mathrm{dm} 3 * 10=1 \mathrm{~L}$

$0530 \quad 0000 \quad 00000000$

05 FRAME $=$ PULSE COUNTER 2 CONFIG

30 NO ERROR / FRAME CONTER $=3$

๑००० WEIGHT $=$ SWITCH

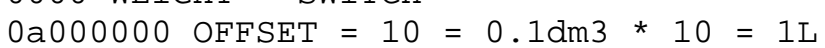


[Adelantado et al., 2017]

[Centenaro et al., 2016]

[Connit, 2018]

[Davoli et al., 2018]

[Djedouboum et al., 2018

[Do et al., 2014]

[ERC, 2018]

[Feltrin et al., 2018]

[Fremantle, 2018]
Adelantado, F.; Vilajosana, X.; Tuset-Peiro, P.; Martinez, B.; Melia-Segui, J.; Watteyne, T. Understanding the Limits of LoRaWAN. IEEE Commun. Mag. 2017, 55, 34-40.

Centenaro, M.; Vangelista, L.; Zanella, A.; Zorzi, M. Longrange communications in unlicensed bands: The rising stars in the IOT and smart city scenarios. IEEE Wirel. Commun. 2016, 23, 60-67.

Connit. Connit Blue Optical Reading System. Available online: http://www.connit.com/en/produit/blue-optical-en/ (accessed on 29 November 2018)

Davoli, L.; Belli, L.; Cilfone, A.; Ferrari, G. From Micro to Macro IOT: Challenges and Solutions in the Integration of IEEE 802.15.4/802.11 and Sub-GHz Technologies. IEEE Internet Things J. 2018, 5, 784-793.

Djedouboum, A.C.; Abba Ari, A.A.; Gueroui, A.M.; Mohamadou, A.; Aliouat, Z. Big Data Collection in LargeScale Wireless Sensor Networks. Sensors 2018, 18, 4474.

Do, Minh-Tien; Goursaud, C.; and Gorce, Jean-Marie. Interference Modelling and Analysis of Random FDMA schemes in Ultra Narrowband Networks, The Tenth Advanced International Conference on Telecommunications, AICT 2014; Paris, France, 20-24 July 2014; pp. 132-137.

European Research Council. ERC Recommendation 70-03 Relating to the use of short range devices. Available online: https://www.ecodocdb.dk/download/25c41779cd6e/Rec7003.pdf (accessed on 29 November 2018).

Feltrin, L.; Buratti, C.; Vinciarelli, E.; De Bonis, R., Verdone, R. LoRaWAN: Evaluation of Link- and System-Level Performance. IEEE Internet Things J. 2018, 5, 2249-2258.

Fremantle, P. A reference architecture for the internet of things. White paper; WSO2 Colombo, Sri Lanka, 2015. Available online: https://ws02.com/download/getfile/wso2_whitepaper_areference-architecture-for-the-internet-of-things.pdf (accessed on 29 November 2018). 
[Ganguli y Friedman, 2005]

[GitHub, 2018]

[Graphite, 2018]

[Guest, 2018]

[IMST, 2018]

[InfluxDB, 2018]

[Itron, 2018]

[ITU, 2005]

[Khutsoane et al., 2017]

[Lauridsen et al., 2017]
IoT Technology Disruptions: A Gartner Trend Insight Report Published: 15 June 2017 ID: G00331334 Analyst(s): Sanjit Ganguli, Ted Friedman.

GitHub. Driver/HAL to build a gateway using a concentrator board based on Semtech SX1301 multi-channel modem and SX1257/SX1255 RF transceivers. Available online: https://github.com/Lora-net/lora_gateway (accessed on 29 November 2018).

Graphite. Graphite Website. Available online: https://graphiteapp.org/ (accessed on 29 November 2018)

Technical overview of Sigfox technology: Network architecture, interfaces, protocol stack. Available online: https://www.survivingwithandroid.com/2018/07/sigfoxprotocol-network-architecture-iot-protocol-stack.html (accessed on 29 November 2018).

IMST GmbH. iC880A LoRaWAN Concentrator $868 \mathrm{MHz}$ Datasheet. Available online: https://wirelesssolutions.de/downloads/RadioModules/iC880A/iC880A_Datasheet_V1_0.pdf (accessed on 29 November 2018).

InfluxDB. InfluxData Website. Available online: https://www.influxdata.com (accessed on 29 November 2018)

Itron Company. Itron Website. Available online: https://www.itron.com/es (accessed on 29 November 2018)

Strategy, I.T.U.; UNIT, Policy. ITU Internet Reports 2005: The internet of things; Geneva: International Telecommunication Union (ITU), Geneva, Switzerland, 2005, 1.

Khutsoane, O.; Isong, B.; Abu-Mahfouz, A.M. IoT devices and applications based on LoRa/LoRaWAN. In IECON 201743rd Annual Conference of the IEEE Industrial Electronics Society, Beijing, China, November 2017; pp. 6107-6112.

Lauridsen, M.; Vejlgaard, B.; Kovacs, I. Z.; Nguyen, H.; Mogensen, $P$. Interference Measurements in the European $868 \mathrm{MHz}$ ISM Band with Focus on LoRa and SigFox. In 2017 IEEE Wireless Communications and Networking Conference (WCNC), San Francisco, United States, 19-22 March 2017; pp. 1-6. 
[Lee y Lee, 2015]

[Libelium, 2018]

[Lloret et al., 2016]

[LoRa Alliance, 2018]

[Margelis et al., 2015]

[Mekki et al., 2018]

[MicroPython, 2018]

[Montginoul y Vestier, 2018]

[Multitech, 2018]

[Pycom, 2018a]

[Pycom, 2018b]
Lee, I.; Lee, K. The Internet of Things (IoT): Applications, investments, and challenges for enterprises. Bus. Horiz. 2015, $58,431-440$.

Libelium. Waspmote Technical Overview. Available online: http://www.libelium.com/products/

waspmote/hardware (accessed on 29 November 2018).

Lloret, J.; Tomas, A.; Canovas A.; Parra L. An integrated IoT architecture for smart metering. IEEE Commun. Mag. 2016, $54,50-57$.

LoRa Alliance. LoRaWAN Specification v1.1. Available online: https://lora-alliance.org/resource-hub/lorawantm-specificationv11 (accessed on 29 November 2018).

Margelis, G.; Piechocki, R.; Kaleshi, D.; Thomas, P. Low Throughput Networks for the loT: Lessons learned from industrial implementations. In Proceedings of IEEE World Forum on Internet of Things, WF-IoT 2015, Milan, Italy, 14-16 December 2015.

Mekki, K.; Bajic, E.; Chaxel, F.; Meyer, F. A comparative study of LPWAN technologies for large-scale IoT deployment, ICT Express 2018, 1, 1-7.

MicroPython community. MycroPython Website. Available online: https://micropython.org (accessed on 29 November 2018).

Montginoul, M.; Vestier, A. Smart metering: A water-saving solution? Consider communication strategies and user perceptions first. Evidence from a French case study. Environ. Modell. Softw. 2018, 104, 188-198.

Multitech. Multitech Website. Available online: https://www.multitech.com/ (accessed on 29 November 2018)

Pycom Company. Website. Available online: https://pycom.io (accessed on 29 November 2018).

Pycom. LoPy4 development board datasheet. Available online: https://docs.pycom.io/datasheets/development/lopy4 (accessed on 29 November 2018). 
[Rahman et al., 2016]

[Raza y Sooriyabandara, 2017]

[Robert Miller, 2018]

[Sanchez-lborra et al., 2018]

[Sanchez-Iborra y Cano, 2016]

[Semtech, 2018a]

[Semtech, 2018b]

[Sigfox, 2018a]

[Sigfox, 2018b]

[Sigfox, 2018c]

[Sigfox, 2018d]
Rahman, A.F.A.; Daud M.; Mohamad, M.Z. Securing sensor to cloud ecosystem using internet of things (IoT) security framework. In Proceedings of the International Conference on Internet of things and Cloud Computing, ACM, Cambridge, UK, 22-23 March 2016, p. 79.

Raza, U.; Kulkarni, P.; Sooriyabandara, M. Low Power Wide Area Networks: An Overview. IEEE Commun. Surv. Tutorials 2017, 19, 855-873.

Robert Miller. MWR Labs Whitepaper, LoRa Security Building a Secure LoRa Solution. Available online: https://labs.mwrinfosecurity.com/assets/BlogFiles/mwri-LoRasecurity-guide-1.2-2016-03-22.pdf (accessed on 29 November 2018).

Sanchez-Iborra, R.; Sanchez-Gomez, J.; Ballesta-Viñas, J.; Cano, M.-D.; Skarmeta, A. Performance Evaluation of LoRa Considering Scenario Conditions. Sensors 2018, 18, 772.

Sanchez-Iborra, R.; Cano, M.-D. State of the Art in LP-WAN Solutions for Industrial loT Services. Sensors 2016, 16, 1.

Semtech Corporation. LoRa overview. Available online: https://www.semtech.com/lora (accessed on 29 November 2018).

Semtech. LoRa ${ }^{\text {TM }}$ Modulation Basics AN1200-22. Available online:

https://www.semtech.com/uploads/documents/an1200.22.pdf (accessed on 29 November 2018).

Sigfox. Sigfox Certification Handbook. Available online: https://build.sigfox.com/steps/certification/\#what-is-a-sigfoxcertification (accessed on 29 November 2018).

Sigfox Company. Website. Available online: https://www.sigfox.com (accessed on 29 November 2018).

Sigfox. M2M and loT redefined through cost effective and energy ptimized connectivity. Available online:

https://lafibre.info/images/3g/201302_sigfox_whitepaper.pdf (accessed on 29 November 2018).

Sigfox. Radio Technology Keypoints. Available online: https://www.sigfox.com/en/sigfox-iot-radio-technology (accessed on 29 November 2018). 
[Sigfox, 2018e]

[Sigfox, 2019]

[Stewart et al., 2010]

[Vejlgaard et al., 2017]

[Voulvoulis et al., 2017]

[Yang et al., 2017]
Sigfox. Sigfox Technology Overview. Available online: https://www.sigfox.com/en/sigfox-iot-technology-overview (accessed on 29 November 2018).

Sigfox. Sigfox Device Radio Specifications. Available online: https://build.sigfox.com/sigfox-device-radio-specifications (accessed on 13 February 2019)

Stewart, R.A.; Willis, R.; Giurco, D.; Panuwatwanich, K.; Capati G. Web-based knowledge management system: Linking smart metering to the future of urban water planning. Aust. Planner 2010, 47, 66-74.

Vejlgaard, B.; Lauridsen, M.; Nguyen, H.; Kovacs, I. Z.; Mogensen, P.; Sorensen, M. Coverage and Capacity Analysis of Sigfox, LoRa, GPRS, and NB-IOT. In 2017 IEEE 85th Vehicular Technology Conference (VTC Spring) (pp. 1-5). Sidney, Australia, 4-7 June 2017.

Voulvoulis, N.; Arpon, K.D.; Giakoumis, T. The EU Water Framework Directive: From great expectations to problems with implementation. Sci. Total Environ. 2017, 575, 358-366.

Yang, W.; Wang, M.; Zhang, J.; Zou, J.; Hua, M.; Xia, T.; You, $X$. Narrowband Wireless Access for Low-Power Massive Internet of Things: A Bandwidth Perspective. IEEE Wirel. Commun. 2017, 24, 138-145. 
- 196 - 